LBL --27233

DE89 014895

\title{
SPECTRAL MEASUREMENTS OF THE COSMIC MICROWAVE BACKGROUND
}

\author{
Alan J. Kogut \\ (Ph. D. Thesis) \\ Lawrence Berkeley Laboratory \\ University of Califomia \\ Berkeley, California 94720
}

April 1989

\section{DISCLAIMER}

\begin{abstract}
This report was prepared as an account of work sponsored by an agency of the United States Government. Neither the United States Government nor any agency thereof, nor any of their employees, makes any warranty, express or implied, or assumes any legal liability or responsibility for the accuracy. completeness, or usefulness of any information, apparatus, product, or process disclosed, or represents that its use would not infringe privately owned rights. Reference herein to any specific commercial product, process, or service by trade name, trademark. manufacturer, or otherwise does not necessarily constitute or imply its endorsement, recommendation, or favoring by the United States Government or any agency thereof. The views and opinions of autiors expressed herein do not necessarily state or reflect those of the United States Government or any agency thereof.
\end{abstract}

This work was supported by the Director, Office of Energy Research, Office of High Energy and Nuclear Physics, Division of High Energy Physics of the United States Department of Energy under Contract Number DE-AC03-76SF00098 and by NSF Grants AST-8406187 and DPP-8716548.

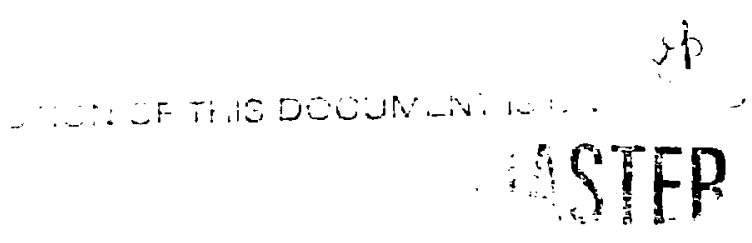


Spectral Measurements of the Cosmic Microwave Background

by

Alan John Kogut

\section{ABstraCT}

Three experiments have measured the intensity of the Cosmic Microwave Background $(\mathrm{CMB})$ at wavelengths $4.0,3.0$, and $0.21 \mathrm{~cm}$ (frequencies $7.5,10$, and 141 $\mathrm{GHz}$ ). The measurement at $4.0 \mathrm{~cm}$ used a direct-gain total-power radiometer to measure the difference in power between the zenith sky and a large cryogenic reference target. Foreground signals (e.g., atmospheric and galactic emission) are measured with the same instrument and subtracted from the zenith signal, leaving the CMB as the residual. The reference target consists of a large open-mouth cryostat with a microwave absorber submerged in liquid helium ( $\mathrm{LHe}$ ); thin windows block the radiative heat load and prevent condensation of atmospheric gasses within the cryostat. The thermodynamic temperature of the CMB at $4.0 \mathrm{~cm}$ is $2.59 \pm 0.07 \mathrm{~K}$ ( $68 \%$ confidence level).

The measurement at $3.0 \mathrm{~cm}$ used a superheterodyne Dicke-switched radiometer with a similar reference target to measure the zenith sky temperature. A rotating mirror allowed one of the antenna beams to be redirected to a series of zenith angles, permitting automated aimospheric measurements without moving the radiometer. A weighted average of 5 years of datz provided the thermodynamic temperature of the $\mathrm{CMB}$ at $3.0 \mathrm{~cm}$ of $2.62 \pm 0.06 \mathrm{~K}$ (68\% confidence level).

The messurement at $0.21 \mathrm{~cm}$ used Very Large Array (VLA) observations of interstellar ortho-formaldehyde to determine the CMB intensity in molecular clouds toward the giant HII region WS1A (G49.5-0.4). Solutions of the radiative transfer problem in the context of a large velocity gradient (LVG) model provided estimates of the CMB temperature within the foreground clouds. Collisional excitation from neutral hydrogen molecules $\left(\mathrm{H}_{2}\right)$ within the clouds limited the precision of the result. The thermodynamic temperature of the CMB at $0.21 \mathrm{~cm}$ is $3.2 \pm 0.9 \mathrm{~K}$ ( $68 \%$ confidence level). 
These results and other recent measurements have provided a more precise determination of the CMB spectrum. Statistical analysis of long-wavelength ground-based result reveals a significant difference of $0.140 \pm 0.034 \mathrm{~K}$ between the ground-based measurements and measurements at higher frequencies using different techniques. The difference can be attributed either to undetected systematic error in either set of measurements (not an uncommon occurrence in the field) or a CMB distortion at wavelenrths $>1 \mathrm{~cm}$. 
TABLE OF CONTENTS

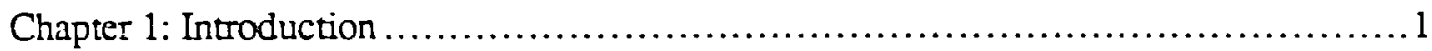

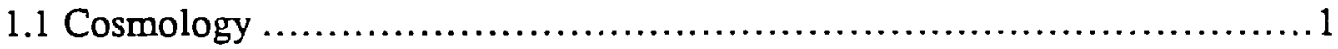

1.2 Testing the Standard Model.....................................................

Chapter 2: Low-Frequency Measurement Concepts ................................ 7

2.1 Foregrounds......................................................... 7

2.2 Definitions and Concepts ........................................... 10

2.3 Observing Strategies........................................................ 10

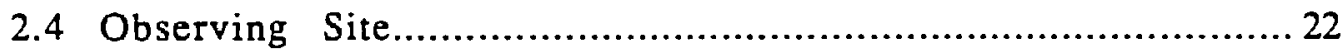

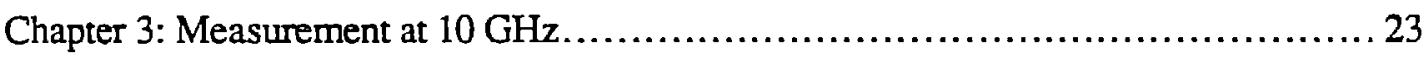

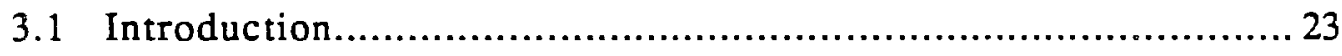

3.2 Tests of System Performance ........................................... 31

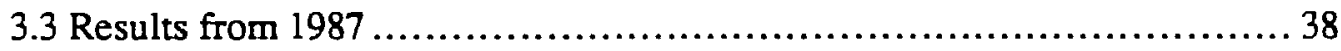

3.4 Overall Results ........................................................ 44

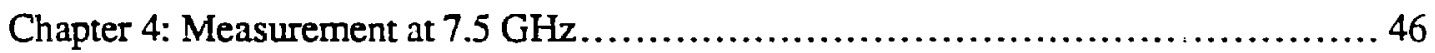

4.1 Introduction............................................................ 46

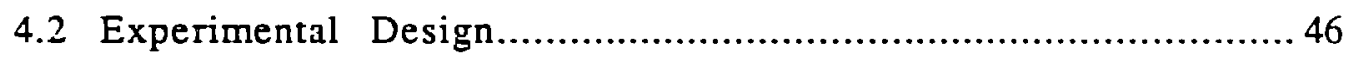

4.3 Data Collection Routines................................................ 56

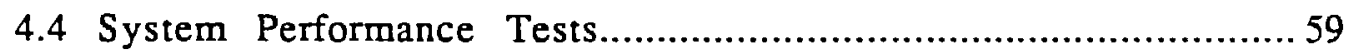

4.5 Data Reduction and Analysis ....................................... 73

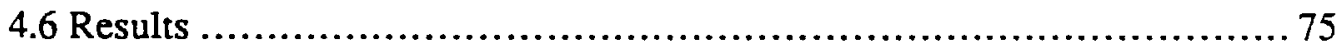

4.7 Discussion ........................................................ 81

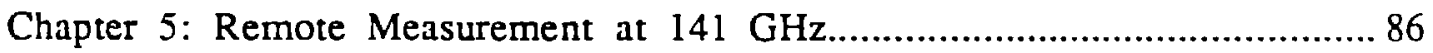

5.1 Introduction........................................................... 86

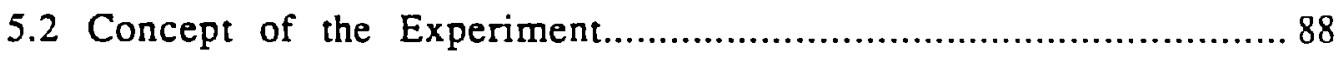

5.3 Observations and Data Reduction ................................... 94

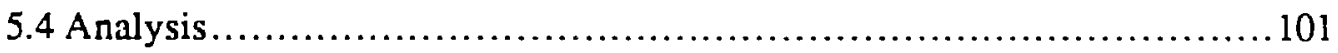

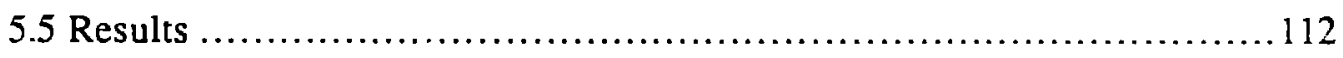

5.6 Discussion ..................................................... 116 
Chapter 6: Analysis and Interpretation........................................ 118

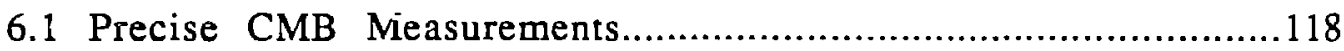

6.2 Mechanisms for Spectral Distortions ................................. 121

6.3 Comparison of Data to Theory ...................................... 126

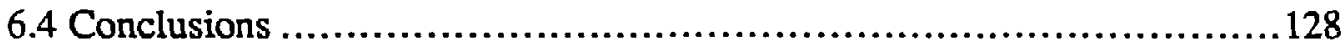

6.5 Future Experiments................................................. 130

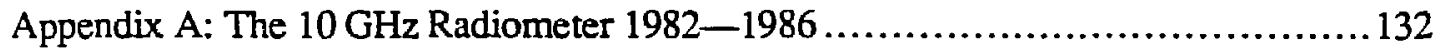

Appendix B: Antenna Temperature of the Reference Load.......................... 175

Appendix C: Interferometry .................................................. 180

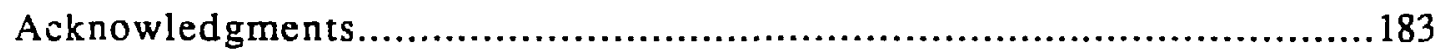

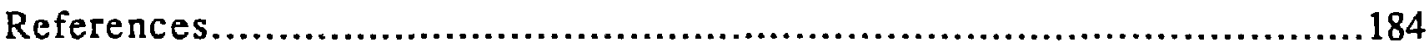




\section{FIGURES}

Figure 2.1 Foreground signals from high-altitude site...................................... 8

2.2 Schematic of cryogenic reference targets....................................... 13

2.3 Components of zenith sky signal ................................... 14

2.4 Schematic of atmospheric tip scans.................................. 17

2.5 Schematic of differential galactic scans....................................... 19

2.6 Schematic of ground shields ............................................ 21

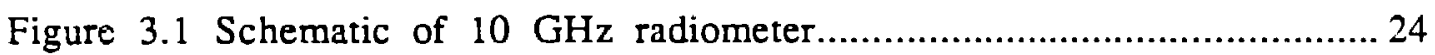

3.2 Schematic of mirror and ground shields ................................. 29

3.3 Method for measuring sidelobe contribution ........................... 33

$3.410 \mathrm{GHz}$ differential galactic profiles................................. 41

3.5 Histogram of $\mathrm{T}_{\mathrm{A}, \mathrm{CMB}}$ measured at $10 \mathrm{GHz}$ in $1987 \ldots \ldots \ldots \ldots \ldots \ldots \ldots \ldots . \ldots \ldots$

Figure 4.1 Schematic of the $7.5 \mathrm{GHz}$ radiometer .............................. 47

4.2 Schematic of the RF chain ............................................. 49

4.3 Far-field beam pattern .................................................. 51

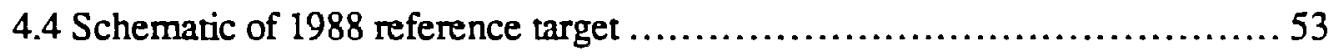

4.5 Temperature of first amplifier as function of time................................ 60

$4.67 .5 \mathrm{GHz}$ differential galactic profile ................................ 76

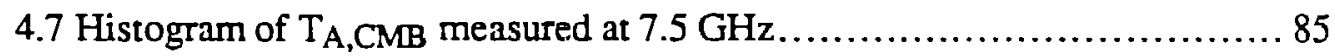

Figure 5.1 Energy levels of cyanogen ........................................ 87

5.2 Energy levels of citho-formaldehyde........................................ 89

5.3 Contour plots of continuum emission toward W51........................... 98

5.4 Contour plots of $6-\mathrm{cm} \mathrm{H}_{2} \mathrm{CO}$ opacity toward W51 ..................... 102

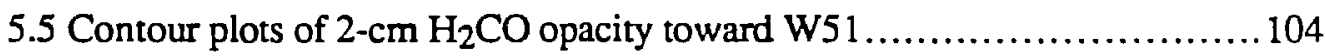

5.6 Opacity spectra toward W51 as function of position ......................106

5.7 Velocity profiles of $\mathrm{H}_{2} \mathrm{CO}$ opacity ................................... 110

Figure 6.1 Recent measurements of CMB spectrum..............................119

6.2 Compton $\mu$-distorted CMB spectrum.....................................131 


\section{TABLES}

Table 3.1 Scatter of $10 \mathrm{GHz}$ atmospheric measurements.. .............................. 32

3.2. Results of sidelobe tests......................................... 32

3.3 Modulation of beam pattern as function of mirtor position ................. 37

3.4 Summary of known systematics ..................................... 37

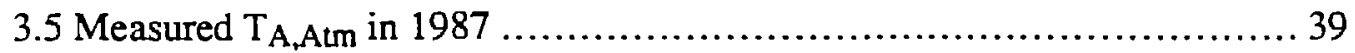

3.6 Comparison of primary and secondary atmospheric scans ................. 39

3.7 Declination and time delay for differential galactic scans.....................43

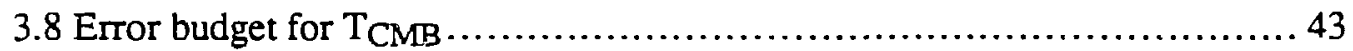

$3.9 \mathrm{CMB}$ thermodynamic temperature at $10 \mathrm{GHz} 1982-87 \ldots \ldots \ldots \ldots \ldots \ldots . .43$

Table 4.1 Properties of reference target........................................... 55

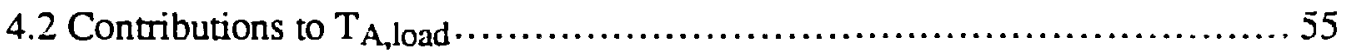

4.3 Measured pointing of atmosphere scan cart...............................55

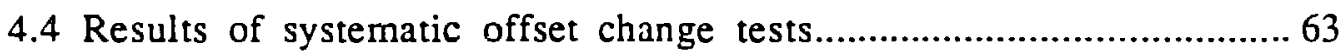

4.5 One-component models of offset changes .............................67

4.6 RMS fluctuations of data as function of lag ............................... 67

4.7 Measured calibration and saturation effects ...............................67 67

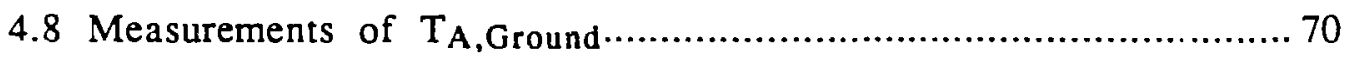

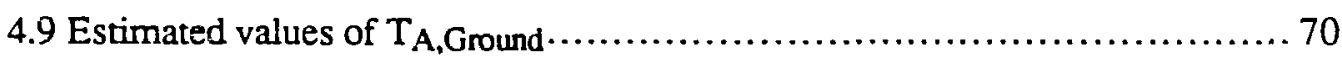

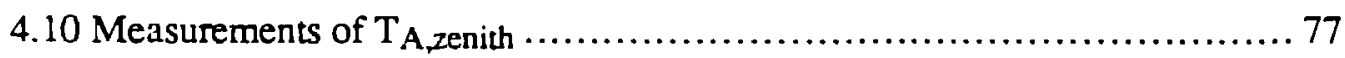

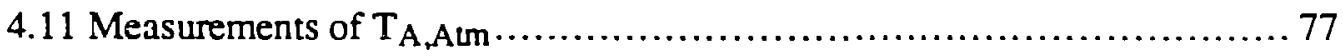

4.12 Contributions to atmospheric error budget ............................. 80

4.13 Contributions to $\mathrm{CMB}$ error budget.................................. 80

4.14 Antenna temperature of atmosphere and CMB...............................82

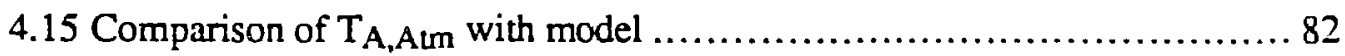

4.16 Results of recent low-frequency CMB rneasurements ................... 82

Table 5.1 Molecular parameters of ortho-formaldehyde......................... 96

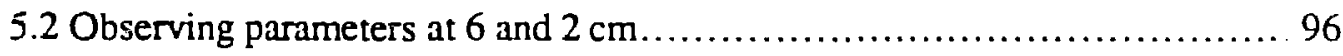

5.3 Peak intensity of brighter continuum regions.............................. 96

5.4 Summary of clump properties..................................... 109

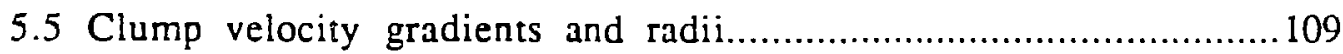

5.6 Derived $\mathrm{H}_{2}$ space density, mass, and velocity .........................113 


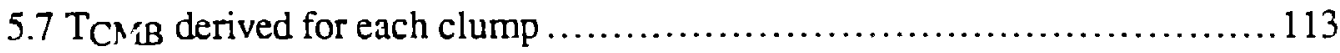

5.8 Systematic contributions to error budget..............................117

Table 6.1 Recent precise $\mathrm{CMB}$ measurements.................................. 120

6.2 Weighted means of various $\mathrm{CMB}$ measurements ....................... 120

6.3 Results of fitting Cc mpton y-distorted spectra ..............................129

6.4 Results of fitting Compton $\mu$-distorted spectra.............................. 129

6.5 Results of various models for spectral distortions at $\lambda<\lambda_{0} \ldots \ldots \ldots \ldots \ldots . . . \ldots 129$

Table A1 Upper limits to systematic offset changes......................................161

A2 Measured gain saturation .......................................... 161

A3 Measured atmospheric antenna temperature ................................ 162

A4 Comparison of 1986 primary and secondary atmospheric measurements......163

A5 Calculated atmospheric antenna temperature ................................ 163

A6 Contributions to atmospheric systematic error........................... 164

A7 Contributions to CMB systematic error............................................164

A 8 Terms used to calculate CMB antenna temperature.............................. 165

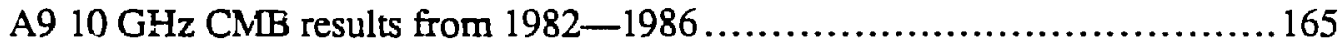

A10 Recent measurements of CMB thermodynamic temperature..................... 166

Table B1 Reflection properties of the reference target ........................... 179

B2 Emission properties of the reference target............................... 179 


\section{Chapter 1 \\ Introduction}

\subsection{Cosmology}

Two of the strongest human longings are the needs to know our future and our past. In this, the astrologers had it backwards: it is not the stars that hold the keys to the future, but the space between the stars that holds the secrets of our past. The discovery and concurrent interpretation of the Cosmic Microwave Background (CMB) as a relic of primordial times profoundly altered observational cosmology: henceforch, investigators of the history of the universe need not be limited to gleaning clues from heavily-processed matter, but could observe the photons which had traveled unimpeded from the early stages of the universe.

The most apparent feature of the right sky, after the sun and moon, is the glowing band of the Milky Way. It was first identified as a great disk of individual stars by Thomas Wright in 1750; however, the extent of the disk was not at all apparent. A clue to that extent lay in the faint fuzzy nebulae, catalogued (as nuisances to be avoided) by Charles Messier in 1781. As telescope technology improved, approximately a third of these objects could be resolved into elliptical shapes, some with spiral arms. Without the ability to resolve individual stars within these objects, the question remained open whether they were distant clouds within our own galaxy, or (as suggested by Immanuel Kant) individual galaxies like our own, best at a great distance.

The first direct evidence of the distance of these nebulae, and hence the size of the universe, came in 1923. Edwin Hubble, using the newly-completed 100-inch telescope at Mt. Wilson, was able to resolve individual stars in the Andromeda galaxy. By observing Cepheid variable stars, whose absolute magnitude is given by their period, Hubble was able to place them at a distance of nearly $10^{6}$ light years, well beyond the boundary of the Milky way. Observational cosmology had begun.

The next great clue to the nature of the universe came in 1929, when Hubble discovered that distant galaxies are receding from us at a velocity proportional to their distance:

$$
\mathbf{v}=\mathrm{H}_{0} \mathrm{~s}
$$


This has a natural interpretation of an expanding universe, and showed that the universe is not the static, unchanging place envisioned previously (various "steady-state" models must continuously create new galaxies to preserve the present mean intergalactic distance). Current estimates for $\mathrm{H}_{0}$ are in the range $50-100 \mathrm{~km} \mathrm{~s}^{-1} \mathrm{Mpc}^{-1}$ (Sandage and Tammann 1976, de Vaucouleurs 1979).

A fundamental assumption, called the Cosmological Principle, is that on sufficiently large scales the universe is isotropic and homogeneous. Consequently, the universe may be described by the Robertson-Walker metric,

$$
d s^{2}=d t^{2}-R^{2}(t)\left\{\frac{d r^{2}}{1-k r^{2}}+r^{2} d \theta^{2}+\left(r^{2} \sin ^{2} \theta\right) d \phi^{2}\right\}
$$

and is governed dynamically by the equation

$$
\mathrm{R}^{2}+k c^{2}=\frac{8 \pi \mathrm{G}}{3} \rho \mathrm{R}^{2}
$$

(e.g., Weinberg 1972). The energy density of the universe is $\rho ; G$ is the gravitational constant. The constant $\mathbf{k}$ describes the curvature of spacetime and is conventionally normalized to \pm 1 or 0 . The distance $s$ between any two points is related to the scale parameter $\mathrm{R}$ and the co-moving distance coordinate $\mathrm{r}$; hence the velocity between two points is given by

$$
\mathbf{v}=\frac{\dot{\mathbf{R}}}{\mathbf{R}} \mathrm{s}=\mathrm{H}_{0} \mathrm{~s}
$$

which defines Hubble's constant $\mathrm{H}_{0}$. The second derivative of the scale factor is given by

$$
\ddot{R}=-\frac{4 \pi G}{3}(p+3 p) R
$$

where $p$ is the pressure. If the quantity $\rho+3 p$ remains positive, the acceleration parameter $\ddot{R} / R$ is negative. Since we observe that $R$ and $\dot{R} / R$ today are both positive, the universe must necessarily have passed through the singular point $R(t)=0$. The time $t$ of the singularity is taken as the origin of the time axis; the subsequent expansion of the universe is the basis of the Big Bang model of cosmology.

Thus far, we have not specified the contents of the universe. The universe today contains baryonic matter in the form of hydrogen and helium; hence protons and neutrons 
must have existed in the early universe. The observed abundance ratio of helium to hydrogen is approximately 0.25 . If the matter in the universe were extremely dense at times soon after the singularity, it would all fuse to helium; consequently, the observed $\mathrm{He} / \mathrm{H}$ abundance indicates the presence of a hot radiation field that could photo-dissociate. deuterium before the fusion occurred. Considerations of this sort prompted Alpher and Herman in 1950 (expanding on the earlier work of Gamow) to predict a "relic" radiation field with a current temperature $-5 \mathrm{~K}$. For primarily sociological reasons, the prediction was allowed to slip into obscurity; it was not until 1964 that A. Penzias and R.W. Wilson accidentally discovered "an excess antenna temperature" of $3.5 \pm 1 \mathrm{~K}$ while measuring the noise properties of a large radio telescope. Their discovery pre-empted a search for the relic radiation by a group at Princeton University by Dicke, Peebles, Roll, and Wilkinson, who published a companion paper suggesting a cosmological origin to Penzias' and Wilson's excess antenna temperature.

In the current "standard model" of the universe's origin, the universe began as a singular point of infinite temperature and density. In the first moments immediately after the expansion, the matter and radiation fields were in close thermal contact. Even the heaviest particles were relativistic $\left(E » \mathrm{mc}^{2}\right)$. From the equation for energy conservation,

$$
\dot{\rho} R^{3}=\frac{d}{d t}\left\{R^{3}(\rho+p)\right\}
$$

with $p=\rho / 3$ for a relativistic gas, the density was proportional to $R(t)^{-4}$. Eq. 1.3 then has the solution

$$
R(t) \sim t^{1 / 2} \text { (radiation dominated) }
$$

Strictly speaking, this solution is valid only for $k=0$; however, for redshifts more than $\sim 100$, the term $k^{2}$ in Eq. 1.3 may be neglected. As the universe expanded, it cooled; as the photon energy dropped below the threshold for pair production, the heavier particles dropped out of number equilibrium. These particles either decayed, annihilated against their anti-particles (provided the expansion rate was not greater than the interaction rate), or survived to the present epoch. Milestones in the radiation-dominated era include

$\begin{array}{lll}\mathrm{T} \sim 10^{11} \mathrm{~K} & .01 \mathrm{sec} & \mu^{+} \mu^{-} \text {annihilation. Neutrinos decouple. } \\ \mathrm{T} \sim 5 \times 10^{9} \mathrm{~K} & 4 \mathrm{sec} & \mathrm{e}^{+} \mathrm{e}^{-} \text {annihilation. Neutron-proton ratio fixed. } \\ \mathrm{T} \sim 10^{9} \mathrm{~K} & 180 \mathrm{sec} & \text { Nucleosynthesis }\end{array}$


As the universe continued to cool, the eivergy density in the photons and neutrinos (the only surviving relativistic particles) fell below that of the matter. In a matter-dominated universe, the density $p-R(t)^{-3}$, and Eq. 1.3 has the solution

$$
R(t) \sim t^{2 / 3} \text { (matter-dominated) }
$$

The universe at this point consisted of a plasma of hydrogen, helium, and free electrons (with neutrinos present but not significantly interacting). At a temperature $-4000 \mathrm{~K}$. $\left(t \sim 7 \times 10^{5}\right.$ years), the electrons and nuclei could form neutral atoms without being photo-dissociated. The plasma "recombined" into a neusal gas, and the opacity of the universe fell to essentially zero.

The photon occupation number $\eta$ for a Planckian spectrum is given by

$$
\eta=\frac{1}{e^{h v / k T}-1}
$$

For an adiabatically expanding universe, the occupation number is invariant. Since the frequency $v$ of the photons scales as

$$
v \sim R^{-1} \sim 1+2,
$$

the temperature $\mathrm{T}$ of the $\mathrm{CMB}$ must also scale in this fashion. Once the $\mathrm{CMB}$ attains a Planckian distribution, further expansion of the universe simply redshifts the distribution without introducing distortions. Since processes such as bremsstrahlung and radiative Compton-scattering are very efficient at establishing a Planckian spectrum in the very early universe, regardless of the initial spectrum, the standard model predicts the CMB today to be a redshifted blackbody spectrum.

Distortions in the CMB spectrum cannot arise simply from the expansion of the universe, and must instead results from the interactions of the radiation and matter fields in the evolving universe. Since the intervening $10-20$ billion years from the Big Bang have seen the matter evolve from an isotropic gas to clustered galaxies, planets, and astrophysicists, the radiation offers a powerful probe of conditions in the early universe. Although the spatial distribution of the radiation probes only the surface of last scattering $(z-1500)$, the spectrum preserves a record of the thermal history from the present to a redshift of a few million, neatly spanning the era in which the universe evolved from homogeneity and isotropy to its present state. 
Energy-releasing processes in the early universe will typically perturb the matter and radiation fields from thermal equilibrium. Interactions between matter and radiation (mainly Compton scattering and thermal free-free processes) will then act to restore equilibrium. If the time from the energy release (at redshift $z_{h}$ ) to the present is shorter than the time scale required to re-establish thermal equilibrium, the CMB spectrum will be distorted from a purely Planckian distribution. Compton-distorted spectra are characterizec by a rise at wavelengths above the peak of the CMB spectrum and a decrease in the Rayleigh-Jeans region. Bremsstrahlung rethermalizes the longest wavelengths up to a frequency dependent on the time available for free-free processes to produce additional photons, causing the spectrum to rise at long wavelengths. Other processes, such as the decay of particles after they fell from equilibrium, can cause an observable signal in the CMB spectrum. The presence or abserice of such signals in the CMB spectrum can provide information on such processes as galactic formation, particle decay, or an eariy generation of massive stars.

\subsection{Testing the Standard Model}

The standard model makes three predictions about the CMB: it should be isotropic (the Cosmological Principle), have a Planck spectrum, and should be redshifted according to $\mathrm{T}(\mathrm{z})=\mathrm{T}_{0}(1+z)$. By the late 1970 's, the CMB spectrum had been confirmed to be roughly Planckian (Weiss 1980 and references therein); however, quite sizable (20\%) distortions could have gone undetected. In 1978, a group at the Lawrence Berkeley Laboratory and Space Sciences Laboratory of the University of Califormia began a program to re-measure the CMB spectrum in the Rayleigh-Jeans region. At the same time, a U.S.Italy collaboration between Haverford, Milano, Bologna, and Padova were planning a similar program. In 1979, the two efforts merged, and subsequently measured the CMB spectrum at wavelengths 12.0,6.3, 3.0, 0.9, and $0.33 \mathrm{~cm}$ (Sironi et al. 1984, Mandolesi et al. 1984, De Amici et al. 1985, Witebsky et al. 1986; Smoot et al. 1983, 1985).

In following years, the Berkeley group continued to improve the techniques of ground-based radiometry, adding new instruments at $20.3 \mathrm{~cm}$ (Levin et al. 1988, Bensadoun et al. 1989), $7.9 \mathrm{~cm}$ (De Amici et al. 1988), and $4.0 \mathrm{~cm}$ (this work). Renewed interest in the field grew sharply after a rocket flight in 1987 observed a large apparent deviation from a Plank spectrum at sub-mm wavelengths (Matsumoto et al. 1988). The 
shape of this distortion (a sharp rise in temperature above the peak of the spectrum) could be modelled by emission from warm dust at redshifts $20 \leq z_{h} \leq 40$, or by a Comptondistortion at $z_{h} \leq 3800 \mathrm{~h}^{-1} \Omega_{\mathrm{b}}-1 / 2$, where $\mathrm{h}$ is the Hubble constant in units of $100 \mathrm{~km} \mathrm{~s}^{-1}$ $\mathrm{Mpc}^{-1}$ and $\Omega_{\mathrm{b}}$ is the energy density of baryonic matter relative to the closure density. These models differ in their effect on the Raleigh-Jeans spectrum: dust models leave the Rayleigh-Jeans spectrum unperturved while Comptonization requires a drop of $\sim 0.2 \mathrm{~K}$. The two low-frequency measurements described in this thesis are part of an effort to characterize the Rayleigh-Jeans spectrum of the CMB.

The third prediction, that the CMB should be hotter at larger redshifts, has not been tested. Interstellar spectroscopy offers a method to probe the radiation field at large distances and remote epochs. By examining molecular absorption lines arising from a common state, the relative populations of the energy levels can be determined, and hence their temperature. The technique has been used very successfully with the cyanogen molecule (CN) within the Milky Way (e.g., Crane et al. 1988, Meyer et al. 1989). Cyanogen, though, nequires a bright star as the source of optical photons to be absorbed, and is unlikely to prove successful in searches at non-zero redshift.

Soon after joining the Berkeley group in 1985, I began work on a project to use interstellar formaldehyde $\left(\mathrm{H}_{2} \mathrm{CO}\right)$ to measure the $\mathrm{CMB}$ temperature at $2.1 \mathrm{~mm}$ wavelength. The observed transitions in formaldehyde are at $\mathrm{cm}$ wavelengths, not in the optical; the illuminating sources are typically large $\mathrm{HII}$ regions instead of single stars. $\mathrm{H}_{2} \mathrm{CO}$ thus offers the hope of an extragalactic measurement of the $\mathrm{CMB}$ temperature as a function of redshift. In this thesis, I will also briefly describe the results of a pilot experiment within our galaxy, which demonstrates the feasibility of using $\mathrm{H}_{2} \mathrm{CO}$ to make measurements (albeit of limited precision) of the CMB. 


\section{Chapter 2 \\ Low-Frequency Measurement Concepts}

\subsection{Foregrounds}

An experimentalist performing direct measurements of the CMB is necessarily limited to sampling that portion of the $\mathrm{CMB}$ which reaches the observing instruments. Since our instruments can occupy only a small fraction of the universe, the observing frequency must be chosen carefully to minimize foreground signais not of cosmological interest. The CMB is a blackbody (to at least the $10 \%$ level) with a temperature of about $2.74 \mathrm{~K}$ across a wide range of frequencies. As such, its intensity peaks at $\mathrm{mm}$ wavelengths, falling off slowly at longer wavelengths (the Rayleigh-Jeans region) and very sharply at shorter wavelengths (the Wien region). At very long wavelengths (a few meters) the falling CMB signal is overwhelmed by galactic and extragalactic synchrotron radio emission, while at very short wavelengths (about 100 microns) the CMB becomes submerged beneath a foreground signal from dust emission. Between these extremes lies the domain of direct $\mathrm{CMB}$ observations, where an experimentalist can avoid or measure the various foreground signals.

The problem of unwanted foreground signals is inherent in the measurement, for the CMB photons must traverse a large path length of matter at temperaturs: irigher than the $\mathrm{CMB}$ itself before reaching us, where they interact with our observing equipment near other large pieces of warm matter (the Earth, moon, and sun). The design of the experiment must address these foregrounds. Some can be eliminated very simply, by shielding (the Earth's emission) or timing (solar and lunar emission), while others can be eliminated only by moving the observing instruments outside the offending matter (the atmosphere), a complicated and costly procedure.

Nature offers a very appealing "window" for direct ground-based CMB measurements in the frequency range between about 1 and $15 \mathrm{GHz}$, where the largest noncosmological foregrounds (the atmosphere and the galaxy) reach a broad minimum. Figure 2.1 shows the contribution of these sources from a high-altitude observing site. Atmospheric emission at microwave frenuencies is dominated by pressure-broadened emission from oxygen $\left(\mathrm{O}_{2}\right)$ and water vapor. Oxygen has lines at 2.5 and $5 \mathrm{~mm}$; at $\mathrm{cm}$ wavelengths its contribution appears as a continuum falling slowly with decreasing frequency. Since oxygen is wellInixed in the atmosphere, its emission is very stable in time. Water vapor has a rotational transition at $22 \mathrm{GHz}$ and causes a sharp rise in the atmospheric signal near that frequency. 

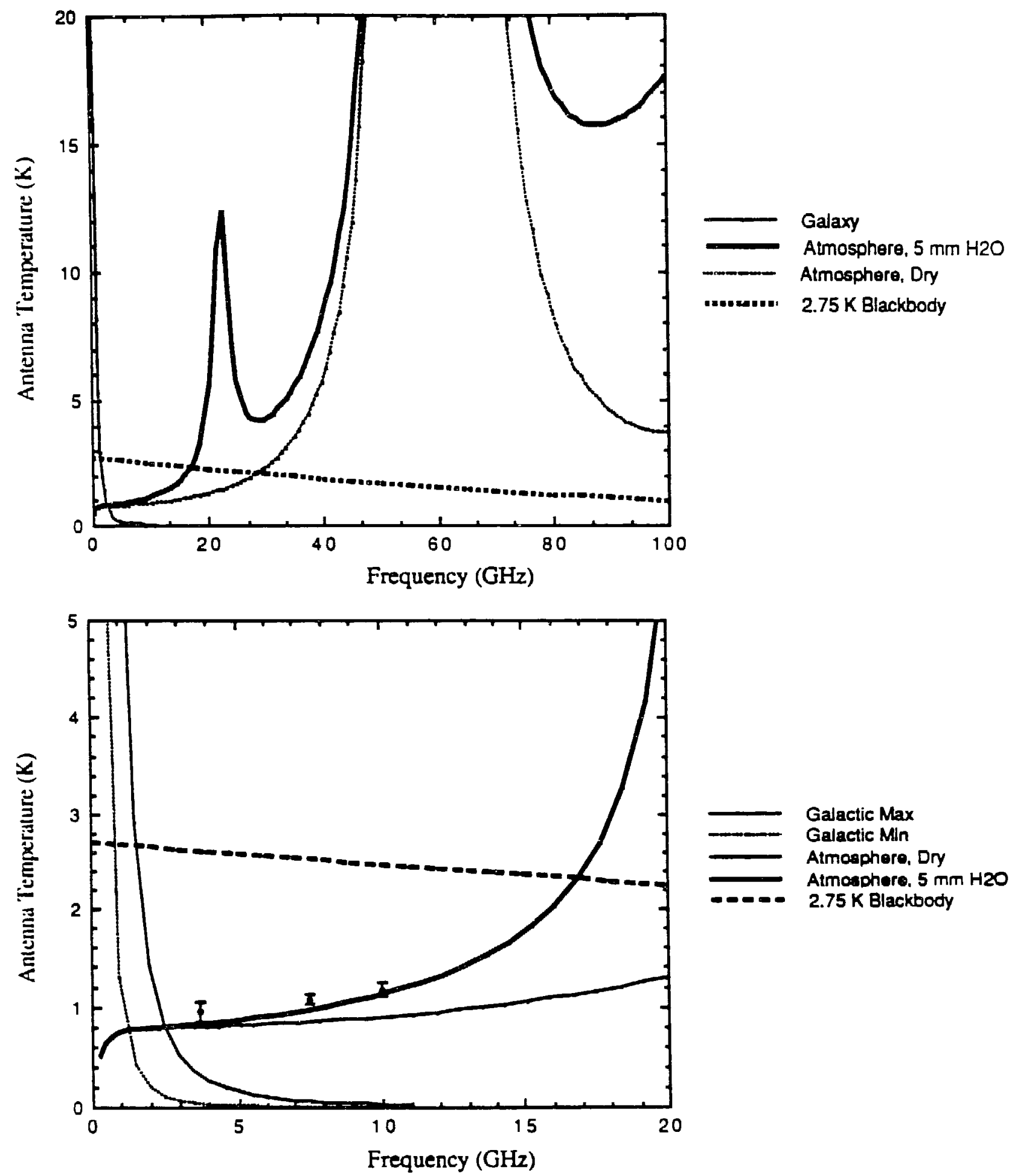

Figure 2.1: Foreground signals from our high-altitude observing site. Typical measured values for the atmosphere are shown. 
The water vapor content of the atmosphere is quite variable, and can cause significant changes in the atmospheric signal on short time scales (several minute to several hours, depending on the frequency of the observation and the local weather conditions). Atmospheric emission reaches minima in magnitude and variability in the spectral regime below $15 \mathrm{GHz}$. At higher frequencies, the experimenter must either plan to accept larger uncertainties in subtracting this signal or resort to balloon or rocket flights with their concomitant complications.

Galactic emission can not be avoided by moving the observing equipment out of the Galaxy, but can be minimized by choice of observing frequency. In the Rayleigh-Jeans portion of the CMB spectrum, Galactic emission results primarily from synchrotron emission and thermal $\mathrm{HII}$ regions. Both processes rise sharply at lower frequencies, with a spectral index $-2.75 \pm 0.1$ for synchrotron radiation and $-2.1 \pm 0.1$ for thermal emission (Webster 1974). Although concentrated in the plane of the Galaxy, synchrotron emission extends in a diffuse component covering the sky, with brightness of order $10 \%$ of the signal in the galactic plane. At frequencies below $800 \mathrm{MHz}$, synchrotron emission becomes the brightest diffuse source in the sky. The upper edge of the Galactic observing window is defined by greybody emission from warm interstellar and interplanetary dust, which, while negligible at $\mathrm{cm}$ wavelengths, dominates the $\mathrm{CMB}$ at wavelengths shorter than a few hundred microns. The combination of a weak galactic signal and a stable atmosphere make the window from 1 to $15 \mathrm{GHz}$ oprimum for direct, ground-based CMB observations. Fortuitously, within this window the wavelength of electromagnetic radiation is on a human scale of several $\mathrm{cm}$, so that the observing equipment can be made of a manageable size.

One signal that certainly is of no cosmological interest is man-made radio-frequency interference (RFI). The microwave spectrum is increasingly populated by a number of transmissions, both military and civilian. The operation and testing of wide-band (BW $1 \mathrm{GHz}$ ) radiometers at frequencies below $15 \mathrm{GHz}$ near major urban centers is rapidly becoming a practice of the past. At present, this problem is still soluble by observing from remote, isolated sites, but it exacts an increasing toll in the developmental phase. 


\subsection{Definitions and Concepts}

At $\mathrm{cm}$ wavelengths, an observed signal is typically within the Rayleigh-Jeans portion of the emitting spectrum. At these wavelengths, signals are conventionally quoted in units of antenna temperature, $T_{A}$, proportional to the power received per unit bandwidth.

$$
T_{A}=\frac{P}{k B}
$$

where $T_{A}$ is the antenna temperanure, $P$ is the received power, $k$ is Boitzmann's constant, and $B$ is the bandwidth of the observation. The antenna temperature is related to the thermodynamic temperature of a blackbody covering the antenna aperture by the relation

$$
\mathrm{T}_{\mathrm{A}}=\frac{\mathrm{x}}{\mathrm{e}^{\mathrm{x}}-1} \mathrm{~T},
$$

where $\mathrm{T}$ is thermodynamic temperature, and $\mathrm{x}$ is the dimensionless frequency

$$
x=\frac{h v}{k T}
$$

where $h$ is Planck's constant and $v$ is the frequency. This convention is particularly useful for ground-based CMB measurements, where the total received signal can contain contributions from greybody emission of warm objects (e.g., the atmosphere), blackbody emission from objects that do not fill the antenna aperture (the moon), and narrow-band signals at low power levels (RFI).

\subsection{Observing Strategies}

\subsubsection{Zenith Sky Measurement}

The low-frequency measurements described herein use radiometers, devices whose output voltage is proportional to the microwave power intercepted by the input (and hence to the antenna temperature of the target viewed). The radiometers use corrugated conical hom antennae to channel radiation efficiently to the receiver input, and to reduce the 
response of the instrument from $4 \pi$ steradians to a more manageable ares. The essence of the experiment is quite simple: to compare the output signal of the radiometer while it alternately views the zenith sky and a reference target whose antenna temperature is well known. From the observed signal difference and the known calibration of the radiometer, the antenna temperature of the zenith sky can be determined. All known sources other than the $\mathrm{CMB}$ are then subtracted from the zenith sky antenna temperature, leaving the CMB as the residual. To minimize effects of calibration uncertainties, the antenna temperature of the reference target should be as close as possible to the antenna temperature of the zenith sky. For ground-based measurements in the $1-15 \mathrm{GHz}$ window, the antenna temperature of the zenibi sky is close to the temperature of liquid helium ( $\mathrm{LHe}$ ); consequently, we use a LHe-cooled target as the reference "cold load."

We calibrate the radiometer by allowing it to view two targets of widely different, known antenna temperatures, and comparing the output difference:

$$
G=\frac{T_{\text {Aload }}-T_{\text {A.Amb }}}{S_{\text {load }}-S_{\text {A mb }}}
$$

where $G$ is the calibration constant of the radiometer, $T_{A, l o a d}$ is the antenna temperature of the LHe-cooled reference target, $T_{A, A m b}$ is the antenna temperature of an ambienttemperature reference target, and $S_{\text {load }}$ and $S_{A m b}$ are the output signals viewing the two targets. In practice, there is also a small correction for saturation effects within the receiver.

The temperature difference between the cold load and the zenith sky is given by

$$
T_{\text {A,zenith }}-T_{\text {A,load }}=G\left(S_{\text {zenith }}-S_{\text {load }}\right) \text {, }
$$

where $T_{A, z e n i t h}$ is the antenna temperature of the zenith and $S_{\text {zenith }}$ is the output signal when the radiometer views the zenith. The zenith antenna temperature is the sum of many signals:

$$
T_{A, \text { zenith }}=T_{A, C M B}+T_{A, A l m}+T_{A, G a l}+T_{A, \text { Ground }}+T_{A, R F I}+\Delta T_{\text {Offset }},
$$


where $T_{A, C M B}$ is the antenna temperature of the $C M B, T_{A, A t m}$ is the antenna temperature of the atmosphere, $T_{A, G a l}$ is the antenna temperature of the Galaxy, $T_{A, G r o u n d}$ is the antenna temperature of the Earth seen in the antenna back- and sidelobes, $T_{A, R F I}$ is the antenna temperature of man-made radio-frequency interference, and $\Delta T_{\text {Offset }}$ refers to any systematic change in radiometer performance in the interval between viewing the zenith and the cold load. Radiation from the sun and moon can, in principle, be present, but are easily avoided by refraining from observations when these sources are above the horizon. Equations 2.5 and 2.6 can be solved for the CMB temperature:

$$
\begin{aligned}
T_{A, C M B}=G( & \left.S_{\text {zenith }}-S_{\text {load }}\right)+T_{A, l o a d} \\
& -T_{A, A t m}-T_{A, G a l}-T_{A, g r o u n d}-T_{A, R F I}-\Delta T_{\text {Offset }}
\end{aligned}
$$

For a LHe-cooled target near $10 \mathrm{GHz}$, the signal difference $\mathrm{G}\left(\mathrm{S}_{\text {zenich }}-\mathrm{S}_{\text {load }}\right)$ is small, typically $0.1 \mathrm{~K}$ or less. The largest term in Eq. 2.7 is the temperature of the reference load, $\mathrm{T}_{\mathrm{A}, \text { load. }}$ To minimize uncertainties in the reference temperature, we use a microwave absorber submerged in LHe within a large $(\sim 70 \mathrm{~cm}$ diameter) upright $(\sim 2 \mathrm{rn}$ tall $)$ cryostat (Figure 2.2). A minimum of material lies between the observing radiometer and the absorber. A metallicized plastic cylinder surrounds the absorber and acts as an oversized multi-mode waveguide ("radiometric wall"), ensuring that the absorber fills the antenna aperture and minimizing emission from warm portions of the cryostat. Thin polyethylene windows at the top of the cryostat prevent air from condensing on the false wall and adding to its emissivity. A fraction of the helium boil-off gas, heated above ambient temperature, circulates between the polyethylene windows to prevent the formation of water condensation or frost on the top window. The antenna temperature of the cold load, to first order, is simply the temperature of the absorber, known from the boiling temperature of LHe and the pressure within the dewar. Small corrections must be made for emission from the radiometric wall and the windows, and from power emitted by the radiometer and reflected back by the various components within the target. The two reference targets used for the two low-frequency measurements are described in greater detail in Appendix A and Chapter 4.

\subsubsection{Atmospheric Measurement}

Within the $1-15 \mathrm{GHz}$ window, the $\mathrm{CMB}$ dominates the zenith sky, as shown in Figure 2.3. The largest subtraction to be made in Eq. 2.7 is the atmosphere; accordingly, the precise measurement of the atmospheric signal occupies the greatest fraction of effort in the low-frequency experiments described in this thesis. Fortunately for ground-based 
Top Plate and

Antenna Aperture

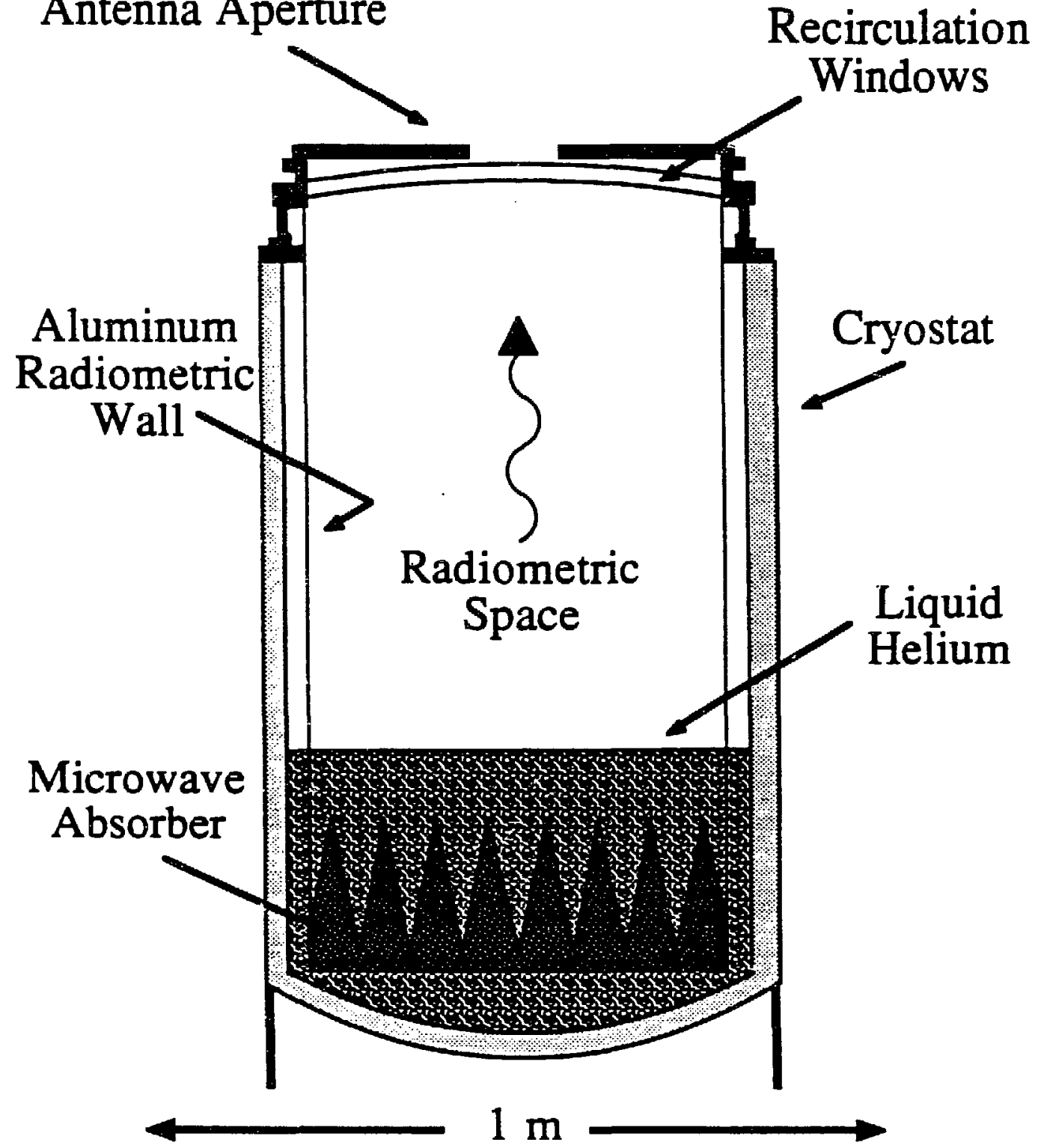

Figure 2.2: Schematic of the large cryogeric reference targets used. 


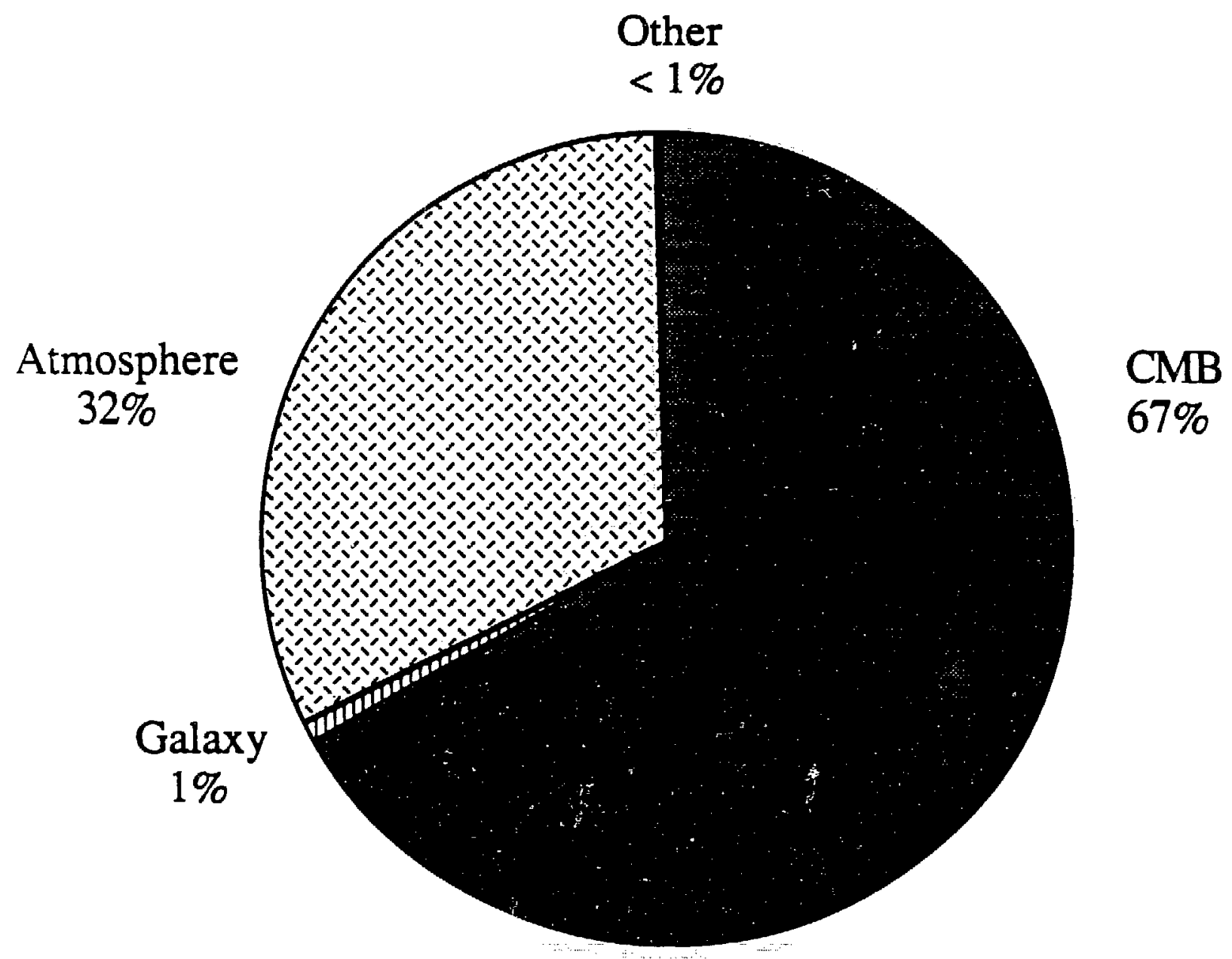

Figure 2.3: Components of the zenith sky signal from our high-altitude observing site. The Galactic signal is the maximum signal on the plane of the Galaxy. 
experiments, the atmosphere at low frequencies is quite isotropic under conditions of clear, cloudless weather. The main source of fluctuations is variation in the column density of water vapor, causing signal fiuctuations of $1 \%$ on angular scales of $80^{\circ}$ or time scales of perhaps half an hour. The precise power spectrum and mechanism of atmospheric scintillation is the topic of some study, but for purposes of wide-beam low-frequency radiometry, it is sufficient to model the atmosphere as an thin, isotropic, homogeneous curved shell covering the surface of the Earth. As such, it can be measured by causing the radicmeter to view varying column densities of atmosphere and attributing the subsequent signal change to the increased airmass in the beam.

The method is indicated schematically in Figure 2.4. For a flat slab-like atmosphere and a delta-function beam pattern, the antenna temperature of the atmosphere at the zenith, $T_{A, A m m}$ may be found from the signal difference between the zenith and any other angle $\theta$.

$$
\begin{aligned}
T_{A, A \text { Am }} & =G \frac{S_{\theta}-S_{\text {zenith }}}{\sec (\theta)-\sec (0)} \\
& =G \frac{S_{\theta}-S_{\text {zenith }}}{\sec (\theta)-1}
\end{aligned}
$$

where $S_{\theta}$ and $S_{z e n i t h}$ are the outputs of the radiometer viewing angle $\theta$ and the zenith, respectively. For angles near the zenith, the denominator becomes very large and has the effect of multiplying noise and small residual signals from non-atmospheric sources into large sources of error. In practice, the angles $\theta=30^{\circ}$ and $\theta=40^{\circ}$ provide equally-spaced denominators of 6.3 and 3.2, respectively. Larger angles provide even smaller secant-theta factors, but greatly increase the difficulties of shielding the radiometer from the warm horizon.

In fact, the above model is a somewhat simplistic. The real atmosphere is curved to follow the Earth's curvature; the beam pattem of the antenna has a non-negligible width of about $12^{\circ}$; the atmospheric opacity causes a slight self-absorption of the signal. It can be shown (e.g. Witebsky et al . 1987, De Amici et al. 1986) that the generalization of Eq. 2.8 is

$$
\mathrm{T}_{\mathrm{A}, \mathrm{atm}}=\frac{\Delta \mathrm{T}_{\mathrm{Atm}}}{\mathrm{F}_{0}}\left\{\mathrm{~F}_{1}+\left(\frac{\Delta \mathrm{T}_{\text {Arm }}}{\mathrm{F}_{0} \mathrm{~T}_{\text {kin }}}\right) \mathrm{F}_{2}+\left(\frac{\Delta \mathrm{T}_{\text {Arm }}}{\mathrm{F}_{0} \mathrm{~T}_{\text {kin }}}\right)^{2} \mathrm{~F}_{3}\right\},
$$

where $\Delta \mathrm{T}$ is the antenna temperature of the signal difference, 


$$
\Delta \mathrm{T}_{\mathrm{Atm}}=\mathrm{G}\left(\mathrm{S}_{\theta}-\mathrm{S}_{\text {zenith }}\right) \text {, }
$$

$T_{\text {kin }}$ is the physical temperature of the atmosphere ( $\left.240 \mathrm{~K}\right)$, and $F_{0}, F_{1}, F_{2}$, and $F_{3}$ are convolutions of the measured beam pattern with a curved atmospheric shell:

$$
\begin{aligned}
F_{0} & =\iint g(z, \phi) H(\theta) d \Omega-\iint g(z, \phi) H(0) d \Omega \\
F_{1} & =\iint g(z, \phi) H(0) d \Omega \\
F_{2} & =\frac{1}{2}\left\{\left[\iint g(z, \phi) H(\theta)^{2} d \Omega-\iint g(z, \phi) H(0)^{2} d \Omega\right] \frac{F_{1}}{F_{0}}-\iint g(z, \phi) H(0)^{2} d \Omega\right\} \\
F_{3} & =\left\{\left[\iint g(z, \phi) H(\theta)^{2} d \Omega-\iint g(z, \phi) H(0)^{2} d \Omega\right] \frac{F_{2}}{F_{0}}\right. \\
& \left.+\frac{1}{6}\left[\iint g(z, \phi) H(0)^{3} d \Omega-\iint g(z, \phi) H(\theta)^{3} d \Omega-\iint g(z, \phi) H(0)^{3} d \Omega \frac{F_{1}}{F_{0}}\right]\right\} .
\end{aligned}
$$

Here $g(z, \phi)$ is the normalized gain pattern of the antenna, and $H(\theta)$ is the path length through the atmosphere at zenith angle $\theta$ :

$$
H(\theta)=\sec (\theta)-\frac{1}{2} \frac{r_{a}}{R_{e}} \sec ^{3}(\theta),
$$

where $R_{e}$ is the radius of the Earth and $r_{a}$ is the scale height of the atmosphere ( $7 \mathrm{~km}$ for $\mathrm{O}_{2}$, the dominant component of atmospheric emission in the $1-15 \mathrm{GHz}$ window). The zenith angle $\theta$ is typically $30^{\circ}$ or $40^{\circ}$. We evaluate the integrais in Eq. 2.14 numerically, using the measured beam patterns of the antennae.

An additional correction must be made to the temperature difference $\Delta \mathrm{T}_{\mathrm{Atm}}$ to account for anisotropic sources of radiation included in the signals $S_{\theta}$ and $S_{z e n i t h}$. Such sources typically include the differential Galactic signal (extrapolated from raps at lower frequencies) and the differential sidelobe pickup of the Earth (typically measured on site). Other corrections may need to be made, as discussed in Chapters 3 and 4 . Since the term $\Delta \mathrm{T}_{\mathrm{Atm}}$ is multiplied by factors of order $3-6$, it is important that any corrections to it be both small and well-understood.

\subsubsection{Jalactic Measurement}

Galactic emission is highly anisotropic and cannot be simply modelled nor subtracted using the results of tip scans from a single radiometer. It can be identified by a 


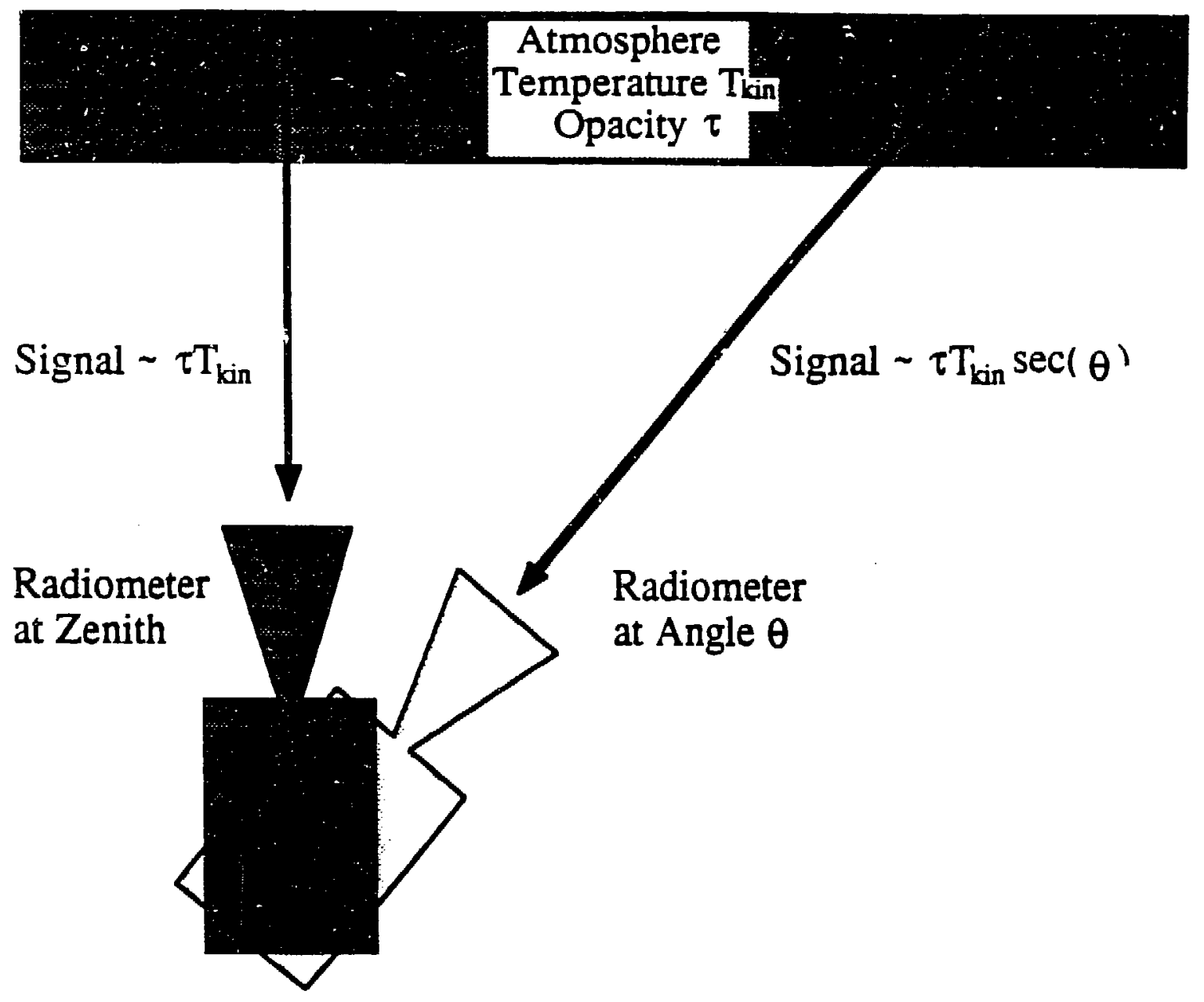

Figure 2.4: Schematic of tip scans used to measure $T_{\text {A.Atm. }}$ 
combination of its angular distribution (concentrated in the Galactic plane) and spectral index ( $-2.75 \pm 0.1$ for synchrotron emission, $-2.1 \pm 0.1$ for emission from Hur regions). By correlating angular and spatial information from several instruments at widely-spaced frequencies, the Galactic signal can be inferred. There is, of course, an ambiguity in that any isotropic Galactic signal cannot be separated a priori from a distortion in the CMB spectrum.

Towards the low-frequency end of the $1-15 \mathrm{GHz}$ window, Galactic emission becomes a limiting factor in the precision of the measurement. The rapid decrease of galactic signal at higher frequencies alleviates this problem for the measurements at 7.5 $\mathrm{GHz}$ and $10 \mathrm{GHz}$ discussed below. Absolute maps of the Galactic brightness exist at 408 $\mathrm{MHz}$ (Haslam et al. 1982) with an estimated error in the zero point of $\pm 4 \mathrm{~K}$. Scaled to 7.5 $\mathrm{GHz}$ with a spectral index of -2.75 , this absolute uncertainty becomes $1 \mathrm{mK}$, negligible for the determination of the zenith sky antenna temperature.

Galactic emission becomes a more serious problem for the tip scans used to determine the atmospheric signal. The Galactic signal rises to $-50 \mathrm{mK}$ in the plane of the Galaxy, which is above the horizon for most of the night during summer months and can easily appear in the atmospheric signal difference $\Delta \mathrm{T}_{\mathrm{Aum}}$. Although the differential uncertainty of the low-frequency Galactic maps is small, the spectral index is not as well known off the plane of the Galaxy. Small uncertainties in the differential Galactic signal are multiplied by factors of 3 or 6 in the atmospheric calculation, and can rise to significant levels. The solution is to perform differential scans of the Galaxy along the same great circle as the atmospheric tip scans. The technique is shown schematically in Figure 2.5. The radiometer is moved approximately twice per minute between two positions at constant zenith angle, and the signal difference berween them determuned. By taking the difference between two positions at constant zenith angle, the atmospheric contribution cancels, and the differential Galactic signal may be found.

$$
\Delta \mathrm{TGal}_{\mathrm{G}}=\mathrm{G}\left[\mathrm{B}\left(\alpha_{1}, \delta_{1}\right)-\mathrm{B}\left(\alpha_{2}, \delta_{2}\right)\right],
$$

where $B$ is the galactic antenna temperature at right ascension $\alpha$ and declination $\delta$. Chopping rapidly between the positions minimizes the effects of small changes in the calibration between the two observations.

Typically, the positions used are $15^{\circ}$ east and $15^{\circ}$ west of the zenith, slightly more than a beam width apart, at constant zenith angle and constant declination. The Earth's 

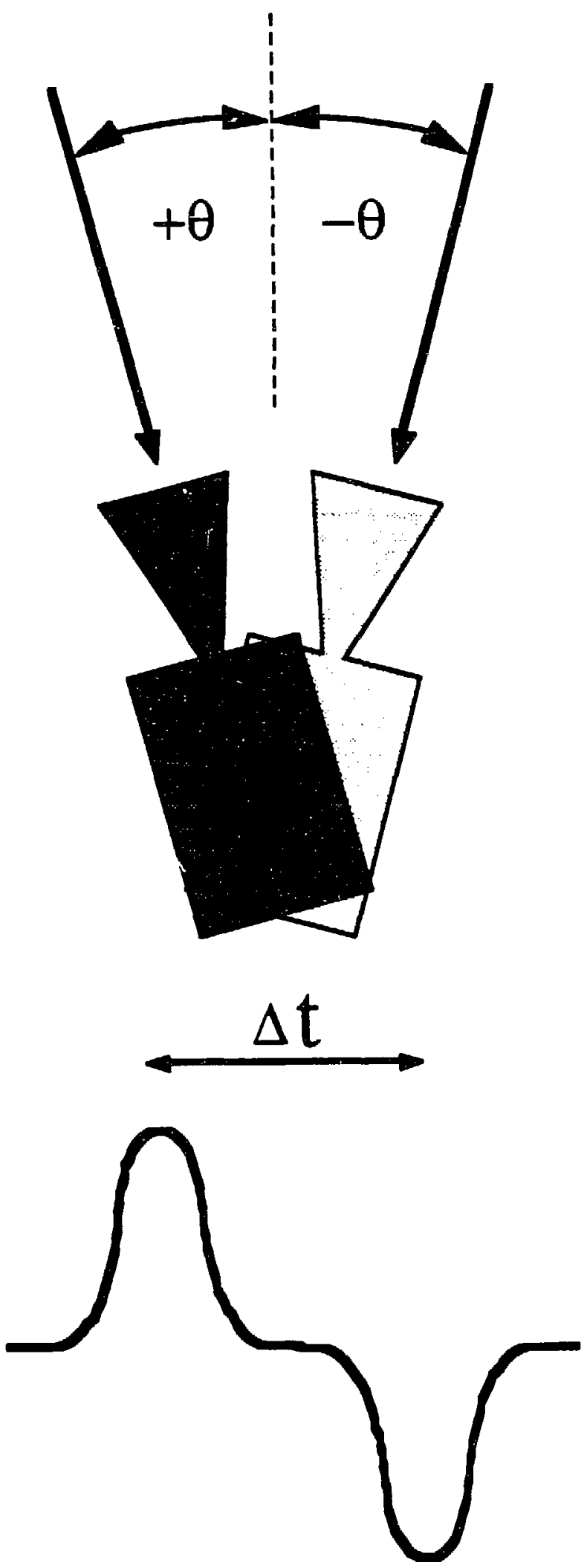

Figure 2.5: Schematic of the differential galactic scans. The differential signal has a double-lobed response to a point source on the sky, indicated at the bottom. The two lobes are separated by a delay $\Delta t$ in right ascension. 
rotation will then csuse any sky signal to appear first in the east beam and two hours later in the west beam. This double signature can be used to distinguish small Galactic signals from noise, provided the radiometer calibration does not change grossly in the intervening hour.

\subsubsection{Sidelobe Reception}

The sky is cold at microwave frequencies, with an antenna temperature near $4 \mathrm{~K}$ in the $1-15 \mathrm{GHz}$ window. The earth occupies fully half of the available $4 \pi$ steradians and is nearly 100 times hotter than the sky. Clearly, some measures must be taken to shield the receiver from radiation from the warm Earth. The first and most obvious step is to use an antenna with a fairly narrow beam, so that the relative gain below the local horizon is small. The two low-frequency experiments described below use the same corrugated hom antenna, designed for use at $10 \mathrm{GHz}$, which has a FWHM of about $12^{\circ}$. The width is small enough to reduce the earth pickup by a factor of $>1500$, yet large enough to dilute small foreground anisotropies; in addition, it allows the antenna to be of reasonable size (30 $\mathrm{cm}$ long) and weight.

Although the antenna gain at angles greater than $90^{\circ}$ is below $-50 \mathrm{~dB}$, the earth still contributes a small signal, of order $0.1 \mathrm{~K}$ depending on the local horizon profile, when the radiometer points toward the zenith. This signal can nearly double when the radiometer is tipped to measure the atmosphere, bringing the horizon closer to the beam center. While the sidelobe response could be modelled given detailed knowledge of the beam pattern and horizon profile, we have chosen to further reduce the sidelobe response by the addition of metal shielding surounding the antenna (Figure 2.6). This shielding acts as a large mirror, redirecting that portion of the beam which would otherwise view the ground to some portion of the cold sky. Ground emission can reach the antenna only after diffracting over the edge of the ground shields. The antenna beam pattern is typically $-35 \mathrm{~dB}$ at the edges of the shield, further reducing diffracted ground pickup. Addition of a flared section or quarter-wave traps on the shield edges can further reduce diffracted ground emission. A well-designed ground shield can reduce the signal from the Earth to less than $0.02 \mathrm{~K}$.

The addition of ground shields to the antenna adds a signal from emission from the shields. This signal is small and independent of radiometer tilt angle. The emissivity of aluminum near $10 \mathrm{GHz}$ is small, typically $3 \times 10^{-4}$, and the gain-weighted area subtended by the shields is also small, typically $0.5 \%$ of the total beam area. The resultant signal 


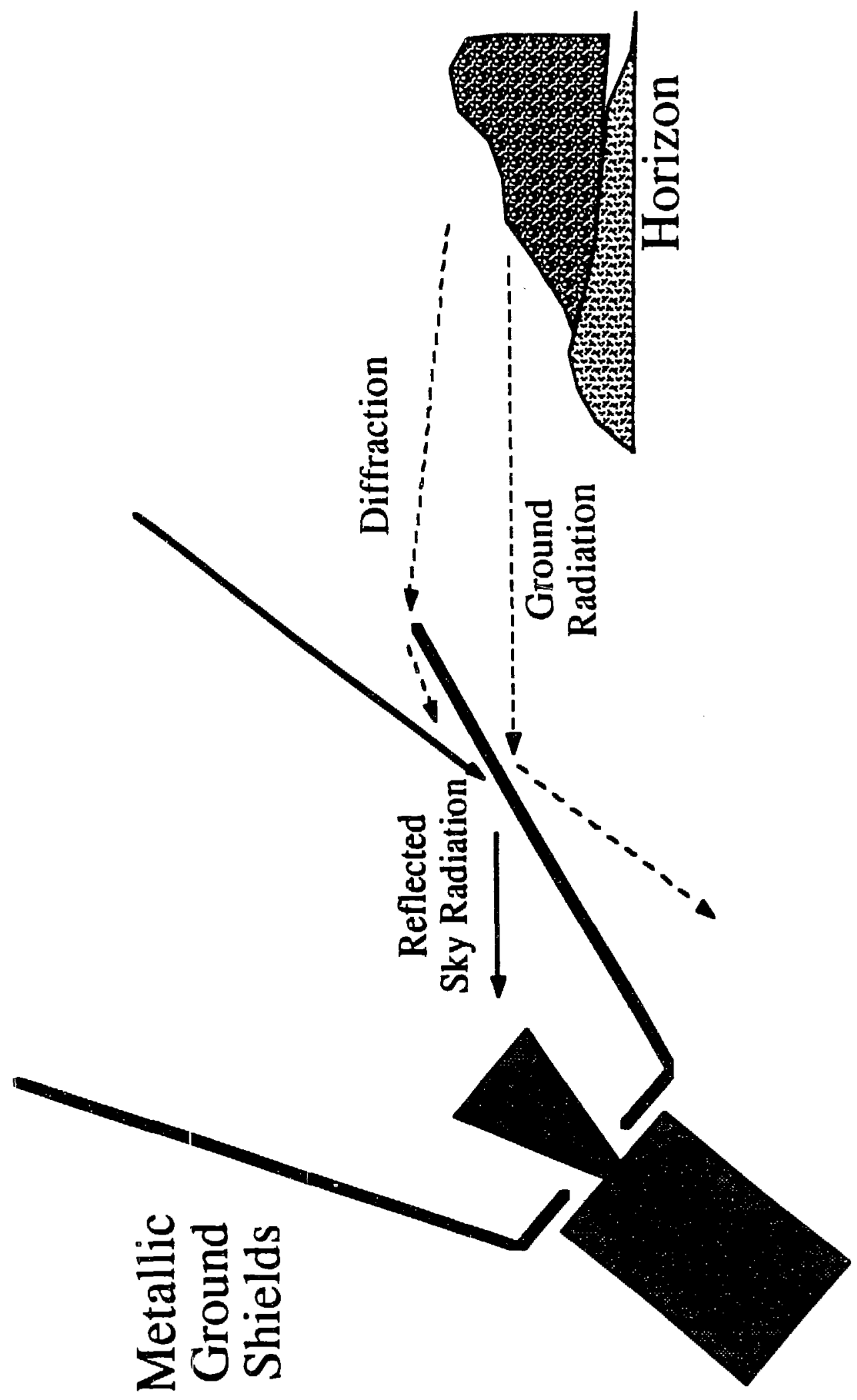

Figure 2.6: Schematic of the ground shields used to reduce reception of radiation from the warm Earth in the antenna sidelobes. 
from shield emission adds $1 \mathrm{mK}$ to the antenna temperature of the sky, and cancels in the determination of the atmospheric signal.

\subsection{Observing Site}

Most of the local foreground signals, particularly atmospheric emission and RFI, can be reduced by observing from a remote, high-altitude site. Our group has made many observations from the Nello Pace Laboratory of the University of California's White Mountain Research Station (latitude $37.5^{\circ} \mathrm{N}$, longitude $118^{\circ} \mathrm{W}$ ). The Station is located on a shoulder of Mt. Barcroft, in the White Mountains of eastem California, at an altitude of $3800 \mathrm{~m}$ (12,500 feet). The area is a high mountain plateau in the rain shadow of the Sierra Nevada range. Typical barometric pressure is $480 \mathrm{~mm} \mathrm{Hg}$, or roughly two-thirds of sealevel pressure. The column density of water vapor is also low, typically in the range $1-5$ $\mathrm{mm} \mathrm{H}_{2} \mathrm{O}$. The altitude and rain shadow effect combine to reduce atmospheric emission and variability compared to sea level by a factor of three at $10 \mathrm{GHz}$, near the center of the $1-15 \mathrm{GHz}$ window.

The nearest sizable population to the Station is the city of Bishop, CA, approximately $25 \mathrm{~km}$ to the southwest in the Owens Valley. The remote location is an extremely important property of Barcroft, for where civilization intrudes, RFI is sure to follow. Major sources of RFI at Barcroft are television satellites, distant radars, microwave relays, and intermittent use of the Station's microwave oven. The frequency range $6.5-11 \mathrm{GHz}$ at this site is largely free of unwanted man-made signals.

Facilities for astrophysics at the site include a hole deep enough to hold the cryogenic reference target, a section of rails laid out to hold radiometers over the reference target in the hole, a small hut to shelter computer and electronic equipment, and (after 1985) line power. Facilities for astrophysicists include a large Quonset hut with a modest shop, sleeping quarters, kitchen, and laboratory space. The local horizon viewed from atop the cold load is far from flat; a large hill rises $18^{\circ}$ toward the west and continues at a slightly lower elevation over most of the northem horizon. The best horizon profile is toward the east, where it is flat and $90^{\circ}$ from the zenith. A large U.S. Air Force base is located in Nevada, in the direction of the best horizon, and is a known source of RFI in the $L$ band $(1.5 \mathrm{GHz})$. 


\section{Chapter 3 \\ Measurement at $10 \mathrm{GHz}$}

\subsection{Introduction}

The international spectrum collaboration formed in 1979 included plans for a radiometer at $10 \mathrm{GHz}(3.0 \mathrm{~cm}$ wavelength), to be built by the Berkeley group. Since that time, the $10 \mathrm{GHz}$ radiometer has become a workhorse of the Berkeley group, participating in $\mathrm{CMB}$ measurements over the course of five summers and at least three incarnations. A number of people contributed to the design of this instrument before $I$ inherited it in the spring of 1986 . A brief review of the tapestry of the $10 \mathrm{GHz}$ radiometer's career is thus in order.

\subsubsection{The Original Instrument}

Scott Friedman built and tested the original instrument (Friedman et al. 1984, Friedman 1984), described in Appendix A. It consisted of a Dicke-switched superheterodyne radiometer with identical comugated conical hom antennas on the two input ports, each with measured half-power beamwidths of $12.5^{\circ}$ in both the $\mathrm{E}$ - and $\mathrm{H}$ planes. The radiometer was mounted on bearings so that one antenna (the "primary" antenna) could rotate to view the zenith sky, atmospheric scan angles of $\pm 30^{\circ}$ (to either side of the zenith), the cold load, or an ambient target. Simultaneously, the other ("secondary") antenna viewed the zenith sky reflected by a large rectangular aluminum mirror mounted at a fixed angle of $45^{\circ}$. This mirror provided the cold, stable target necessary for the proper operation of a Dicke-switched radiometer. The output of a Dicke-switched radiometer is proportional to the difference in microwave power intercepted by the two input ports. The $100-\mathrm{Hz}$ switching between two homs viewing targets of similar temperature minimized the effects of small gain fluctuations, greatly improving the stability of the radiometer at the cost of increased complexity and greater radiometer noise. A measurement consisted, then, of the double difference between the radiometer output with the primary viewing one target and the output when the primary viewed a second target, and assumed that the signal from the secondary arm was constant and independent of the position of the primary. The "basic radiometer" from 1982 remained essentially unchanged throughout the next five years. It is shown schematically in Figure 3.1. The major changes involving the radiometer took place external to the RF chain, changing the method used to measure the variable atmospheric signal. 


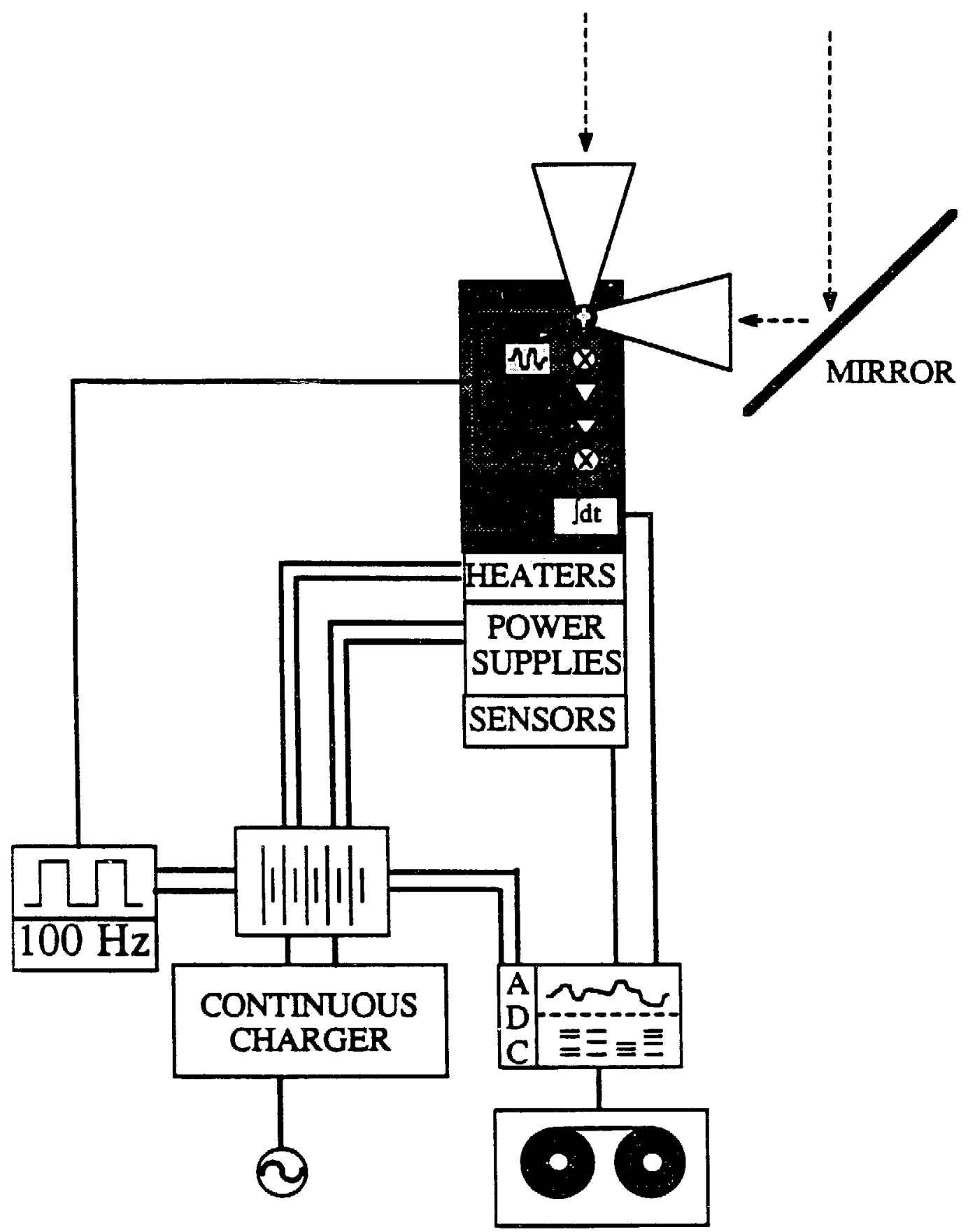

Figure 3.1: Schematic of the radiometer and support equipment. 
In 1982, the primary antenna performed atmospheric scans by viewing the zenith and angles $30^{\circ}$ to either side. A small wire mesh shield surounded the primary to minimize sidelobe pickup of the nearby hillside. The secondary antenna viewed a flat, rectangular aluminum miror, using a small quarter-wave plate to reduce the signal modulation induced by the elliptical polarization of the sky radiation reflected from the metal mirror. A smaller set of wire mesh shields surrounded the secondary horn and miror. The measurements of the $\mathrm{CMB}$ using the cold load and the atmospheric tip scans were performed within a minute of each other, as the primary hom altemately rotated to view the cold load and each sky angle. The radiometer made the journey to Barcroft for the first time in 1982, and performed measurements of the CMB on July 5 and 6 .

\subsubsection{Modifications in 1983}

Although in principle the atmosphere can be measured adequately using scan angles of only $30^{\circ}$, the multiplicative factor of 6 from the secant $(\theta)$ dependence required tighter limits on systematic angle-dependent changes in radiometer performance than were practicable. Accordingly, Scott modified the mounting in 1983 to allow scan angles of $\pm 40^{\circ}$ in addition to the existing $\pm 30^{\circ}$ angles. To accommodate the larger zenith angles, the mesh shields surrounding both the primary antenna and the secondary antenna/mirror grew slightly larger, a trend that would continue throughout the lifetime of the instrument. Scott and the radiometer made the trip to Barcroft a second time, and re-measured the CMB on September 4, 5, and 6,1983.

\subsubsection{First Attempts at Scanning Mirror: 1984}

Analysis of the data from 1983 showed a serious discrepancy between the 1982 values of the atmosphere and the 1983 values, attributable to undetected position-dependent offset changes during the 1982 atmospheric scans. In 1983, the Berkeley group decided to make radical changes in the system for measuring the atmosphere, to allow the secondary antenna to scan independently of the primary. Prior to 1984, the secondary viewed the sky through a large fixed mirror while the primary (and the entire radiometer) rotated to view each angle in turn. If the mirror, though, could be made to rotate about an axis coaxial with the symmetry axis of the secondary horn, it would cause the secondary beam to scan the sky while the radiometer itself remained motionless (the primary antenna, viewing the zenith, would then provide the stable reference target for a Dicke radiometer). Having the miror scan while the radiometer remaired motionless would eliminate the suspected culprit 
behind the 1982-83 discrepancy, would greatly reduce the systematic error budget of the experiment, would provide an independent check of the atmospheric temperature calculated from the still-operational primary scans, and (a point not lost on weary astrophysicists) could be automated, freeing a human being for other duties during the arduous observing nights on Barcroft.

By this time, Scott had graduated and left the University; the task of adding a scanning mirror to replace the fixed one fell to a group including undergraduates Mark Griffith and Bruce Grossan. The mirror chosen was one originally used for automated atmospheric scans at $9.8 \mathrm{GHz}$ by the Haverford group led by R.B. Partridge (Partridge et al. 1984). The new secondary mirror was an ellipse of $1.3 \mathrm{~cm}$ thick aluminum, mounted to rotate about the center of the ellipse instead of a focus. An opto-electronic circuit and a thin metal disk with pinholes every degree about the circumference, mounted on the mirror drive shaft, provided mirror control: by masking or unmasking holes, the mirror could be made to stop at any angle. After a programmable time (typically 32 seconds), the mirror advanced to the next position. A potentiometer mounted on the mirror shaft provided position readout for later analysis. To accommodate the heavy mirror and drive motor, the cart holding the entire assembly was extended one meter.

The elliptical mirror, mounted approximately one meter from the seconidary antenna aperture, intercepted $99.7 \%$ of the secondary beam. After travelling a meter, reflecting off the mirror, and traveling another meter or two, the secondary beam diverged considerably before leaving the vicinity of the radiometer and its cart. The larger beam required a considerably expanded set of shields about the secondary horn/mirror combination. The 1984 configuration used larger sheet-metal shields on the sides of cart, extending past the secondary antenna and the mirror. A large $\left(8^{\prime} \times 6^{\prime}\right)$ wire mesh shield, mounted behind the mirror, redirected the residual mirror spillover from the ground to the zenith.

A problem associated with a scanning mirror is that of alignment: a stationary mirror need only redirect the beam to a stable position, but a scanning mirror must redirect the beam to a known position. It is crucial that the mirror's axis of rotation be coaxial with the antenna's symmetry axis. If the axes are parallel but offset, the mirror will intercept a variable fraction of the secondary beam as the mirror rotates. If the mirror center is located on the antenna symmetry axis but the mirror axis is not parallel to the symmetry axis (and the ground), the redirected beam will not point to the proper scan angles. The mirror mount could be translated and pivoted about all three spatial axes, until the axes were aligned, as 
checked by a laser, a beam splitter, ind a small optical mirror mounted at the center of the secondary antenna mouth.

The radiometer, in its new incarnation, made another trip to Barcroft in the summer of 1984. The primary antenna performed atmospheric scans as in the past and scanned angles of $\pm 40^{\circ}$ and $\pm 30^{\circ}$. The secondary antenna made its first atmospheric scans in 1984, viewing angles of $\pm 30^{\circ}, \pm 40^{\circ}, \pm 47^{\circ}$, and $\pm 54^{\circ}$, chosen to provide nearly equal increments in atmospheric airmass. The larger zenith angles of the secondary scans made operation of the radiometer impractical on the rails extending from the buried cold load, as the horizon to the west was quite higher than that to the east. Instead, the radiometer was moved periodically between the rails and a small platform located about $15 \mathrm{~m} \mathrm{SW}$ of the cold load, used in previous years by an atmospheric monitor at $9.4 \mathrm{GHz}$ (Partridge et al. 1984). The platform allowed the radiometer to be oriented NE-SW to take advantage of the nearly equal horizon profiles in those directions. The radiometer performed atmospheric scans with both the primary and secondary from the platform. During CMB measurements, all available hands carried the radiometer to the rails, where it performed primary atmospheric scans as part of the usual CMB measurement over the cold load. The ground shields and mirror alignment used for secondary scans in 1984 proved inadequate, however, and the scanning mirror system provided no useful data that year.

\subsubsection{A Working System: 1986}

I joined the Berkeley group in 1985, a year in which we did not make the customary summer observations from Barcroft, and in which both Bruce and Mark graduated. Early in 1986, we began laying plans for the summer trip to Barcroft, where we would test our new generation of low-frequency, total-power radiometers. There were two primary scientific motivations for investing a significant amount of time to upgrade the $10 \mathrm{GHz}$ radiometer before the trip. First, a new measurement of the atmosphere at $10 \mathrm{GHz}$ would allow a re-determination of the $\mathrm{CMB}$ temperature with significantly reduced uncertainties. In a field dominated by systematics, it is often invaluable to repeat a measurement using radically different equipment and techniques. Second, we needed a reliable atmospheric monitor that could measure the atmospheric temperature throughout the night with minimal human intervention. The resultant temperature profiles would be used to cross-check the values at lower frequencies, and would differentiate between periods of atmospheric stability and occasional periods of drift. 
During the spring and early summer of 1986 , I modified the scanning mirror system to the point where it would provide useful and reliable atmospheric data. This required several major modifications. Tests from 1984 to 1986 showed that the wire mesh ground shields, viewed at normal incidence, emitted significantly (up to $10 \mathrm{~K}$ ). Part of this was undoubtedly the result of oxidation of the now nearly five year old mesh shields. By late summer 1986, new shields made of solid sheet aluminum had replaced the old mesh shields surrounding the secondary antenna and mirror, including new shields between the radiometer cart and the ground. The secondary antenna/mirror system was now enclosed in a roughly bowl-shaped aggregation of shields, blocking it completely from the ground but leaving it open to the sky. The shields to the sides acquired both quarter-wave traps on the outside edges and a set of "diffraction" shields to replace the ground radiation diffracting at the edge of the side shields with reflected radiation from the sky. In the new configuration (Figure 3.2), ground radiation had to diffract at least twice before entering the secondary beam. The primary antenna also acquired a new set of shields, used during measurements of $\mathrm{T}_{\mathrm{A} \text {,zenith }}$ over the cold load. During secondary atmospheric scans, we continued to use the smaller wire mesh shield around the primary to prevent movement of nearby personnel from modulating the reference signal from the primary.

The new mirror had to be integrated with the primary scanning system, which was still needed to measure the zenith sky temperature. When assembled in 1986, the radiometer consistently produced a systematic offset change of $0.1 \mathrm{~K}$ as the primary rotated with an ambient target firmly attached to the antenna aperture. This "flip offset" resılt from a slight misalignment of the radiometer on its bearings. A 0.003 radian angle between the symmetry and rotation axes of the secondary antenna caused the secondary beam to wander slightly across the undersized elliptical mirror. Since the mirror need not rotate during primary scans, I built a large trapezoidal minor, which fit snugly over the elliptical mirror and between the various shields. This successfully removed the flip offset observed with the radiometer.

Our experience in 1984 had confirmed the choice of scan angles at $\pm 30^{\circ}, \pm 40^{\circ}, \pm 47^{\circ}$, and $\pm 54^{\circ}$. A new digital mirror angle readout replaced unreliable analog system of 1984 . The rebuilt radiometer, armed with software to allow real-time analysis of atmospheric scans, made its fourth journey to Barcroft in August, 1986. Tests in Berkeley and at Barcroft of the new system bolstered our confidence to the point where we modified the CMB measuring technique of previous years. In 1986, the secondary scans provided the best determination of the atmospheric temperature. Prior to the zenith temperature 


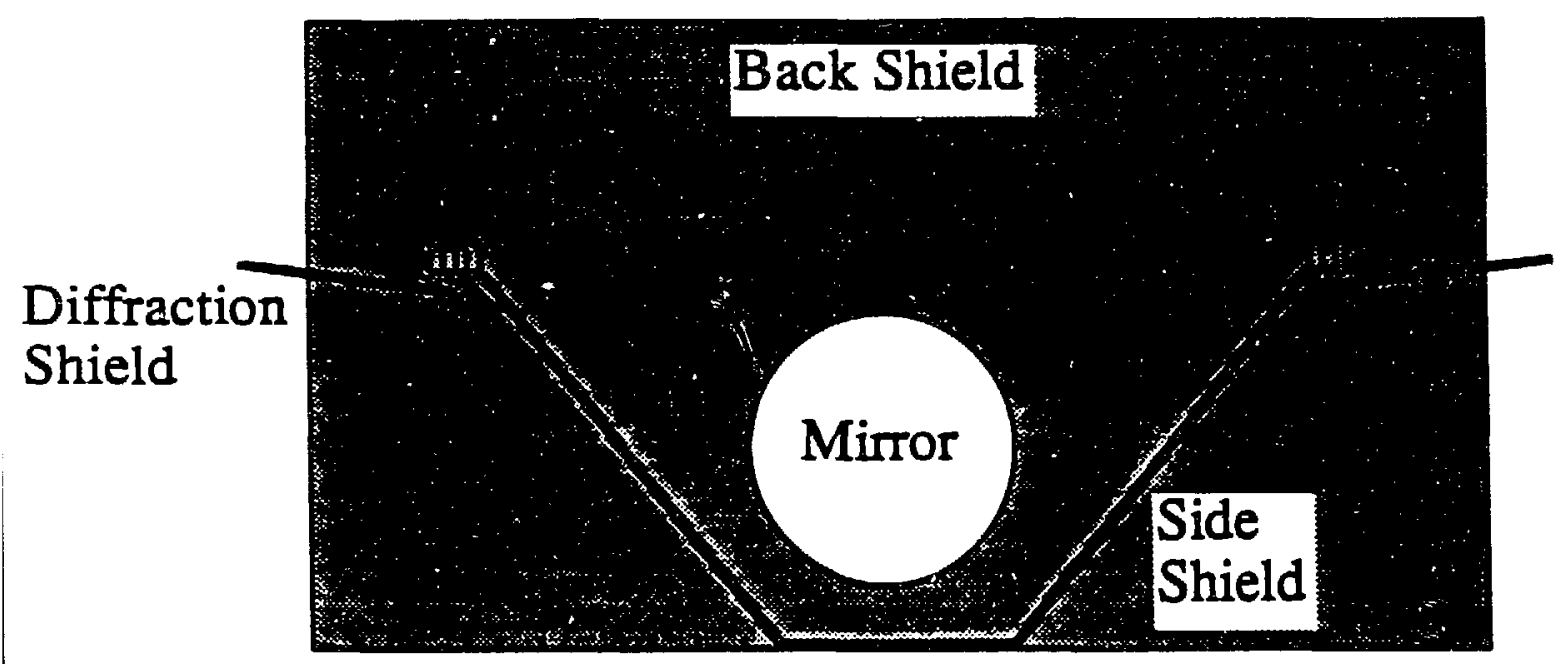

Figure 3.2: Schematic of the mirror and ground shield configuration as viewed from the secondary antenna. The elliptical mirror, mounted at $45^{\circ}$, appears circular in projection. Quarter-wave traps and diffraction shields reduce diffracted ground radiation over the side shields. The back shield is at a $45^{\circ}$ zenith angle (see also Appendix A). 
measurement, the radiometer spent a few hours automatically measuring the atmosphere. We then stopped the sequence, carried the radiometer to the rails, and used the primary antenna to measure the zenith sky (and the atmosphere as a precaution) over the cold load. After the run over the cold load we carried the radiometer back to the platform, where it resumed atmospheric scans for the remainder of the night. We obtained the value for $\mathrm{T}_{\mathrm{A} \text {,Aum }}$ during the determination of $\mathrm{T}_{\mathrm{A}, \text { zenith }}$ by linear interpolation of the secondary scans performed before and after the measurement.

The data from 1982 - 86 have been reported elsewhere (Kogut et al. 1988, reprinted as Appendix A). The conversion to secondary atmospheric scans was successful in that it allowed the atmospheric temperature to be ditermined to higher precision than in previous years. There were still problems, however, in that the atmospheric antenna temperatures at each of the eight secondary angles showed asymmetric systematic displacements from each other, with measurements on one side of the radiometer systematically hotter than the other, and with larger zenith angles systematically hotter than lower angles. In the hope of removing these residual systematic effects, we determined to hurl the radiometer into the breach one last time, and return to Barcroft after another year of testing and development.

\subsubsection{Modific stions in 1987}

Table 3.1 summarizes the observed systematic differences in the 8 determinations of $\mathrm{T}_{\mathrm{A}, \mathrm{Atm}}$. There are two distinct features: a dependence on zenith angle, with larger zenith angles systematically warmer, and an asymmetry, with angles on one side of the radiometer systematically warmer than the same angle on the other side. Modifications and tests in 1987 were attempts to isolate and remove the cause of these behaviors.

Suspicion centered on three main effects: interference from the wire mesh shield surrounding the primary antenna, sidelobe response, and modulation of the spillover from the mirror. To combat these, we removed the primary mesh shield (Appendix A), replaced the diffraction shields with a larger set of quarter-wave traps, and replaced the metal shield behind the elliptical mirror with one $50 \%$ larger. We also modified the lockin amplifier to allow the operator to switch the DC gain from its nominal value ("low gain") to a scale 25 times more sensitive ("high gain"). By changing the signal difference $S_{\theta}-S_{z e n}$ at the ADC from some 20 digitized units (d.u.) to 500 d.u., we could rule out the data digitization/recording system as the cause of the systematic effect. 
Thus modified, the radiometer underwent a regimen of tests in Berkeley before making its final joumey to Barcroft in September, 1987. The results of the tests and the limits derived on suspected systematic effects are reported below.

\subsection{Tests of System Performance}

\subsubsection{Sidelobe Response}

The zenith-angle dependence of the values for $T_{A, A t m}$ measured by the rotating mirror looked suspiciously like a sidelobe effect. The complexity of the shield system and the large size of the beam as it left the cart area made simple modelling difficult We spent a great deal of effort in ruling out sidelobe response as a major contributor to systematics in the radiometer.

We used three types of tests. With the radiometer in a set configuration, we alternately placed and removed an additional "chop" shield from one side of the cart while recording the digitized data stream. The shield spent a fixed time, usually 16 or 32 seconds, in each position. A typical test lasted 17 minutes and covered 32 or 16 full periods (chop shield in place followed by chop shield removed). Subsequent analysis with a digital computer allowed us to search for signals synchronous with the chopping period, with typical RMS noise of a few $\mathrm{mK}$.

The first test searched for evidence that the side shields were too small, allowing undiffracted radiation from the hillside to enter the secondary beam (Figure 3.3a). In this test (an "extension test"), we held the extra chop shield as a simple extension of the side shields, in effect extending them an extra meter. If the side shields were, in fact, too small, the chop shield would block some fraction of the direct radiation; the resultant signal would show a square wave synchronous with the chopping period with amplitude equal to the amount of the blocked radiation, and phase such that the signal was smaller with the chop shield in place. Instead, we typically observed a square wave with the opposite phasemore radiation entered the beam with the extension than without it. This is the result expected if there is no direct path to the beam - the observed signal is the result of diffraction over the combined shields moving closer to the beam center and hence higher in the antenna gain pattern. 
(a)
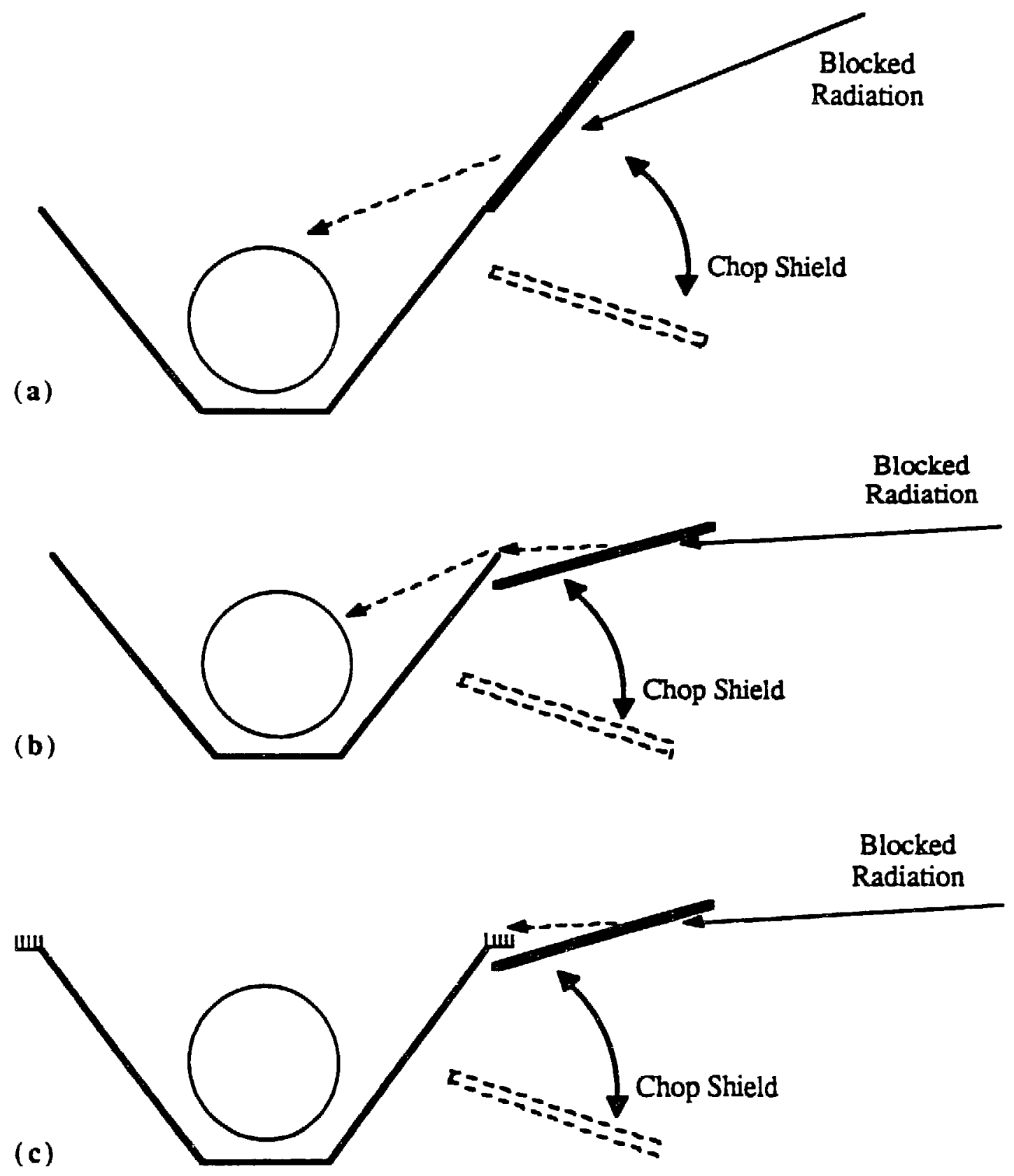

Figure 3.3: Schematic representation of the tests limiting contribution of $T_{A, G r o u n d}$. The aluminum chop shield was altemately placed to block a radiation path, then removed. 


\begin{tabular}{|c|c|c|c|c|c|c|c|c|}
\hline Date & $+54^{\circ}$ & $+47^{\circ}$ & $+40^{\circ}$ & $+30^{\circ}$ & $-30^{\circ}$ & $-40^{\circ}$ & $-47^{\circ}$ & -54 \\
\hline \multicolumn{9}{|c|}{1986 Data } \\
\hline $\begin{array}{l}\text { Aug } 6 \\
\text { Aug } 7 \\
\text { Aug } 8 \\
\text { Aug } 9\end{array}$ & $\begin{array}{l}21 \\
39 \\
59 \\
56 \\
44\end{array}$ & $\begin{array}{c}9 \\
20 \\
51 \\
23 \\
34\end{array}$ & $\begin{array}{l}17 \\
13 \\
64 \\
-8 \\
-9\end{array}$ & $\begin{array}{c}133 \\
-9 \\
25 \\
-56 \\
-85\end{array}$ & $\begin{array}{c}-89 \\
-53 \\
-101 \\
-45 \\
-34\end{array}$ & $\begin{array}{c}-64 \\
-40 \\
-61 \\
-13 \\
-2\end{array}$ & $\begin{array}{c}-36 \\
-5 \\
-28 \\
3 \\
19\end{array}$ & $\begin{array}{l}13 \\
38 \\
-9 \\
44 \\
30\end{array}$ \\
\hline$<1986\rangle$ & $\begin{array}{c}44 \\
(15)\end{array}$ & $\begin{array}{c}39 \\
(15)\end{array}$ & $\begin{array}{c}15 \\
(30)\end{array}$ & $\stackrel{2}{(85)}$ & $\begin{array}{l}-64 \\
(29)\end{array}$ & $\begin{array}{l}-36 \\
(28)\end{array}$ & $\begin{array}{l}-9 \\
(23)\end{array}$ & $\begin{array}{c}23 \\
(21)\end{array}$ \\
\hline \multicolumn{9}{|c|}{1987 Data } \\
\hline $\begin{array}{l}\text { Sep } 15 \\
\text { Sep }_{11} \\
\text { Sep } 18 \\
\text { Sep } 19 \\
\text { Sep } 20\end{array}$ & $\begin{array}{l}53 \\
51 \\
71 \\
86 \\
66 \\
73 \\
69\end{array}$ & $\begin{array}{c}-17 \\
3 \\
22 \\
35 \\
-2 \\
3 \\
3\end{array}$ & $\begin{array}{l}-84 \\
-60 \\
-10 \\
-31 \\
-74 \\
-61 \\
-77\end{array}$ & $\begin{array}{l}-157 \\
-81 \\
-11 \\
-61 \\
-127 \\
-110 \\
-104\end{array}$ & $\begin{array}{c}-14 \\
0 \\
-46 \\
-51 \\
21 \\
-21 \\
-16\end{array}$ & $\begin{array}{c}62 \\
-4 \\
-78 \\
-50 \\
5 \\
-26 \\
4\end{array}$ & $\begin{array}{c}74 \\
32 \\
0 \\
12 \\
30 \\
35 \\
25\end{array}$ & $\begin{array}{c}87 \\
59 \\
52 \\
61 \\
82 \\
104 \\
94\end{array}$ \\
\hline$<1987\rangle$ & $\begin{array}{c}67 \\
(12)\end{array}$ & $\begin{array}{c}7 \\
(17)\end{array}$ & $\begin{array}{l}-57 \\
(27)\end{array}$ & $\begin{array}{l}-93 \\
(48) \\
\end{array}$ & $\begin{array}{l}-18 \\
(25)\end{array}$ & $\begin{array}{c}-12 \\
(45)\end{array}$ & $\begin{array}{r}30 \\
(23) \\
\end{array}$ & $\begin{array}{r}77 \\
(20) \\
\end{array}$ \\
\hline
\end{tabular}

Table 3.1: The difference (in $\mathrm{mK}$ ) between measurements of $\mathrm{T}_{\mathrm{A} . \mathrm{Atm}}$ at each angle and the mean of all eight angles. The data are presented from individual days from Aug. 6-9 1986 and Sept. 15-20 1987. Rows labelled <1986> and <1987> (bold type) are averages over the preceding dates, with the RMS scatter shown in parentheses below each mean. The radiometer was disassembled and the mirror re-aligned between 1986 and 1987.

\begin{tabular}{lcccccccc}
\hline Test & $+54^{\circ}$ & $+47^{\circ}$ & $+40^{\circ}$ & $+30^{\circ}$ & $-30^{\circ}$ & $-40^{\circ}$ & $-47^{\circ}$ & $-54^{\circ}$ \\
\hline Extension & $-18 \pm 3$ & $-5 \pm 3$ & $3 \pm 3$ & $3 \pm 3$ & $-1 \pm 3$ & $-1 \pm 3$ & $-21 \pm 4$ & $-104 \pm 4$ \\
Diffraction & $21 \pm 3$ & --- & $3 \pm 3$ & --- & --- & --- & -- & $18 \pm 3$ \\
Trap & $-5 \pm 6$ & --- & $1 \pm 3$ & --- & $\ldots$ & $3 \pm 3$ & $\ldots$ & $-2 \pm 3$ \\
\hline \hline
\end{tabular}

Table 3.2: Results of three types of sidelobe tests. Each entry is the difference, in mK, between the signal with the chop shield removed and the signal with the chop shield in place. The sign is positive if the chop shield blocks radiation. 
A second test measured the extent of diffraction over the side shields (a "diffraction test"). With no quarter-wave traps on the side shields, we alternately placed and removed the chop shield below the side shields and at a shallower angle (Figure 3.3b). In this way, we replaced ground radiation diffracting at the edge of the side shield with reflected sky radiation. With the chop shield in place, the diffracted signal should be reduced by the ratio of the sky temperature to the ground temperature, $(3.5 \mathrm{~K} / 270 \mathrm{~K})=0.01$. The expected square wave would have an amplitude equal to $99 \%$ of the ground diffraction, and would appear colder when the chop shield was in place. This is generally what we observed.

Once we had ascertained that diffraction existed without the quarter-wave traps, we re-tested the system with the quarter-wave traps in place ("trap test"). This third test was quite similar to the second, with the exception that this time the quarter wave traps (or diffraction shields in 1986) were left in place (Figure 3.3c). The extra shield, chopped below the quarter-wave traps, should again produce a signal equal to $99 \%$ of the ground radiation that normally would enter the secondary beam past the quarter-wave traps. No such signal was observed, indicating that the quarter-wave traps were blocking essentially all of the signal observed in the diffraction tests.

The results of sidelobe tests in 1986 and 1987 are summarized in Table 3.2. The extension test and the diffraction test are consistent with a $20 \mathrm{mK}$ diffraction signal. The anomalously high value for the extension test at $-54^{\circ}$ can be attributed to the higher horizon $\left(18^{\circ}\right)$ to the $(-)$ side of the radiometer. The null results of the trap test indicate that the quarter-wave traps succeed in their purpose of preventing this $20 \mathrm{mK}$ signal from reaching the secondary beam.

\subsubsection{Mirror Spillover}

The elliptical mirror is slightly undersized and intercepts most but not all of the secondary antenna beam. We tested for modulation of the beam pattern on the mirror as a function of mirror angle by completely covering the mirror with an ambient absorber (Eccosorb CV-3). After purting all shields in place, we left the absorber to thermalize for a period of three hours. We then rotated the mirror, spending 64 seconds at each allowed position (eight angles plus the zenith). The results did indeed show a small signal modulation, summarized in Table 3.3. The mirror intercepted the greatest fraction of the beam in the $+54^{\circ}$ position, and the least at $-54^{\circ}$, but the magnitude of the modulation was far too small to explain the atmospheric offsets. From Table $3.2, \mathrm{~T}_{\mathrm{A}, \mathrm{Atm}}$ at $54^{\circ}$ is some 50 $\mathrm{mK}$ warmer than the mean, requiring the signal $\Delta \mathrm{T}_{\mathrm{Alm}}$ to be positive by $50 \mathrm{mK}$ * 
[sec $\left(54^{\circ}\right)-1$ ], or about $35 \mathrm{mK}$. For a $0.2 \%$ modulation in total beam area to produce so large a signal would require a source of magnitude $15 \mathrm{~K}$, either from an increased sky signal at $54^{\circ}$ or a decrease in a warm background at the zenith. The $0.4 \mathrm{~K}$ increase in sky temperature at $54^{\circ}$ fails to meet the required $15 \mathrm{~K}$ source magnitude by a factor of 37 . The spillover past the mirror views a background of the zenith sky, reflected by a large metal shield, and is both too small and of the wrong sign (the spillover increases, not decreases, at the zenith) to explain the effect. Simple modulation of the beam by the rotating mirror can not be the cause of the atmospheric offsets.

\subsubsection{Electrical Interference}

It was possible that the mirror drive motor could produce a ground loop dependent on motor position. and hence on mirror angle. The signal offsets required to produce the offsets in $T_{\text {A,am }}$ could be produced in 1986 by a $1 \mathrm{mV}$ position-dependent ground loop. A signal-average of a test in which an ambient absorber covered the secondary hom while the mirror rotated showed no signal at the $7 \mathrm{mK}$ level. In addition, atmospheric scans in 1987 ran the DC amplifier on a scale 24 times more sensitive than in 1986, but showed atmospheric offsets of similar magnitude. A ground loop from the motor would be expected to inject a signal after the DC amp, typically in the $20 \mathrm{~m}$ BNC cable running from the $\mathrm{DC}$ amp to the $\mathrm{ADC}$ /multiplexer. Since the atmospheric offset remained constant on two different gain scales, the source must lie upstream of the DC amp, effectively ruling out the motor.

\subsubsection{Gain Ratio}

The signal differences between the zenith and the cold load, or the zenith and the sky at one of the scan angles, are small, typically below $0.4 \mathrm{~K}$. The output signal difference was correspondingly small, of order $12 \mathrm{mV}$. The $20 \mathrm{~m}$ of BNC cable connecting the radiometer to the data recording system allowed some susceptibility to ground loops. As a cross-check, the radiometer in 1987 included a feature allowing the gain of the DC amplifier to be switched between two scales: the "low" scale, which mapped an input range of $\pm 6.4 \mathrm{mV}$ to an output range $\pm 10 \mathrm{~V}$, and a "high" scale, which mapped the range $\pm 200 \mu \mathrm{V}$ to the output. Data from Barcroft in 1987 were taken almost exclusively at high gain.

Unfortunately, the calibration signal from an ambient Eccosorb target was off-scale at high gain. Calibrating the radiometer at high gain required a somewhat circuitous procedure. We desire the calibration constant at high gain, 


$$
G_{\text {high }}=\frac{T_{1}-T_{2}}{\left(S_{1}-S_{2}\right)_{\text {high }}}
$$

but can only measure directly the calibration at low gain,

$$
\mathrm{G}_{\text {low }}=\frac{\mathrm{T}_{1}-\mathrm{T}_{2}}{\left(\mathrm{~S}_{1}-\mathrm{S}_{2}\right)_{\text {low }}}
$$

Here $S_{1}$ and $S_{2}$ are the output signals corresponding to target temperatures $T_{1}$ and $T_{2}$. To determine $\mathrm{G}_{\text {high }}$ we measured the ratio of two gain scales by measuring the signal difference between the zenith sky and a greybody emitter (a thin piece of cardboard) whose signal remained on-scale for both high and low gain settings. The ratio $\alpha$ of signal differences is the ratio of the two differential calibration constants:

$$
\alpha=\frac{G_{\text {high }}}{G_{\text {low }}}=\frac{\left(S_{1}-S_{2}\right)_{\text {high }}}{\left(S_{1}-S_{2}\right)_{\text {low }}}
$$

For atmospheric scans, the two targets used for calibration are an ambient Eccosorb target and the zenith sky, with the calibration constant determined by

$$
\mathrm{G}_{\text {high }}=\alpha \frac{\mathrm{T}_{\text {A,amb }}-\mathrm{T}_{\mathrm{A}, \text { zenith }}}{\left(\mathrm{S}_{\mathrm{amb}}-\mathrm{S}_{\text {zenith }}\right)_{\text {low }}},
$$

where $T_{A, z e n i c h}$ is taken to be the sum

$$
\mathrm{T}_{\mathrm{A}, \text { zenith }}=\mathrm{T}_{\mathrm{A}, \mathrm{CMB}}+\mathrm{T}_{\mathrm{A}, \mathrm{Atm}}
$$

and solved iteratively for each atmospheric scan. Measurements of $T_{A, z e n i c h}$ using the reference cold load did not need to iterate, as there were two known temperatures: the cold load and the ambient target. As with the atmospheric scans, we observed the zenith sky at both gain scales, but with the zenith antenna temperature known from Eq. 2.5. Solving Eqs. 2.5, 3.3, and 3.4 for $\mathrm{G}_{\text {high }}$ yields the solution

$$
\mathrm{G}_{\text {high }}=\frac{\overline{\mathrm{A}}_{\mathrm{A}, \mathrm{Amb}}-\mathrm{T}_{\mathrm{A}, \text { load }}}{\alpha^{-1}\left(\mathrm{~S}_{\mathrm{amb}}-\mathrm{S}_{\text {zenith }}\right)_{\text {low }}-\left(\mathrm{S}_{\text {load }}-\mathrm{S}_{\text {zenith }}\right)_{\text {high }}},
$$

which contains only observables. Eq. 3.6 could have been simplified by observing the cold load at both gain scales rather than the zenith sky, but we wished to minimize the IR heat leak to the load and kept observations of the cold load to a minimum. 


\begin{tabular}{lcccccccc}
\hline Angle & $+54^{\circ}$ & $+47^{\circ}$ & $+40^{\circ}$ & $+30^{\circ}$ & $-30^{\circ}$ & $-40^{\circ}$ & $-47^{\circ}$ & $-54^{\circ}$ \\
\hline \% Change & 0.23 & 0.21 & 0.17 & -0.07 & -0.07 & -0.05 & -0.05 & -0.07 \\
From Zenith & $(0.03)$ & $(0.03)$ & $(0.03)$ & $(0.03)$ & $(0.03)$ & $(0.03)$ & $(0.03)$ & $(0.03)$ \\
\hline
\end{tabular}

Table 3.3: Modulation of the beam pattern as a function of mirror position. The number in parentheses under each entry is the $68 \%$ C.L. statistical uncertainty of the measurement.

\begin{tabular}{|c|c|c|c|}
\hline Effect & Magnitude & $\begin{array}{l}\text { Uncertainty in } \\
\mathrm{T}_{\mathrm{A}, \mathrm{Atm}}(\mathrm{mK})\end{array}$ & $\begin{array}{l}\text { Uncertainty in } \\
\mathrm{T}_{\mathrm{A}, \text { zenith }(\mathrm{mK})}\end{array}$ \\
\hline $\begin{array}{l}\text { Systematic Offset, Warm Target } \\
\text { Systematic Offset, LN Target } \\
\mathrm{T}_{\text {A.load }} \\
\text { Calibration Ratio } \\
\text { Gain Saturation } \\
\text { Galactic Signal } \\
\text { Ground Loops } \\
\text { Ground in sidelobes } \\
\text { Pointing Accuracy } \\
\text { Beam Pattern } \\
\text { Beam Spillover }\end{array}$ & $\begin{array}{c}0 \pm 20 \mathrm{mK} \\
-2 \pm 8 \mathrm{mK} \\
3568 \pm 13 \mathrm{mK} \\
24.44 \pm 0.13 \\
1.019 \pm 0.001 \\
8 \pm 4 \mathrm{mK} \\
0 \pm 7 \mathrm{mK} \\
0 \pm 3 \mathrm{mK} \\
\pm 4^{\prime} \\
\pm 3^{\circ} \text { FWHM } \\
0.2 \%\end{array}$ & $\begin{array}{c}-- \\
\cdots \\
- \\
6 \\
1 \\
19 \\
23 \\
10 \\
6 \\
25 \\
2\end{array}$ & $\begin{array}{l}20 \\
13 \\
<1 \\
<1 \\
4 \\
7 \\
3 \\
-- \\
--\end{array}$ \\
\hline Total & & 41 & 25 \\
\hline
\end{tabular}

Table 3.4: Summary of known systematics. The uncertainty in each effect is shown for the measurement of $\mathrm{T}_{\mathrm{A}, \mathrm{Aum}}$ at $40^{\circ}$ (column 3 ) and for $\mathrm{T}_{\mathrm{A}, \text { zenich }}$ (column 4). Offsets associated with moving the radiometer do not apply to the atmospheric measurements as the radiometer remains motionless during secondary scans. We take the larger uncertainty of the warm target as an upper limit to offset changes in the measurement of $T_{A, z e n i t h}$. 
We measured the gain ratio $\alpha$ many times and found it to be quite stable. The mean gain ratio at Barcroft in 1987 was $\alpha=24.44 \pm 0.13$. Uncertainty in the high-gain calibration contributed negligibly to the determination of $T_{A, A t m}$ and $T_{A, z e n i t h}(5 \mathrm{mK}$ ).

\subsubsection{Other System Tests}

We performed a number of other tests to determine the magnitude of known effects contributing to the error budget. They are discussed in detail in Appendix A. The results in 1987 were of similar magnitude to those of 1986, and are summarized in Table 3.4. We were unable to determine the cause of the systematic atmospheric effects observed in 1986 and again in 1987 , as discussed below.

\subsection{Results from 1987}

\subsubsection{Atmospheric Antenna Temperature}

The radiometer measured the antenna temperature of the atmosphere from Sept. 9 through Sept. 211987 (UT), as summarized in Table 3.5. Between Sept 9 and Sept 15, the secondary antenna/mirror alignment was changed several times in an effort to reduce the systematic atmospheric off sets. The radiometer was unchanged from Sept 15-21. Clear weather prevailed for all scans except Sept 12 and to some extent Sept 14 and 15, when a strong frontal system moved through the area.

The data from the eight individual scans angles display a systematic offset after all known corrections to the data have been made. The data in which the radiometer configuration is unchanged (Sept 15-21) have a mean spread of $211 \pm 17 \mathrm{mK}$, with $-54^{\circ}$ generally the warmest and $+30^{\circ}$ the coldest. The RMS scatter of the eight angles about the mean of each observation over the same time period is $71 \pm 5 \mathrm{mK}$. It is evident that some systematic effect of unknown origin is affecting at least some of the eight angles; however, we have no a priori knowledge whether the effect is to raise or lower the affected angles. If the lowest angle $\left(+30^{\circ}\right)$ is correct, we err by $108 \pm 15 \mathrm{mK}$ by using the mean of all eight angles; if, on the other hand, the highest angle $\left(-54^{\circ}\right)$ is correct, we err by $-83 \pm 6 \mathrm{mK}$ by using the mean. Lacking any better indicator, I have chosen to take the RMS scatter of the eight angles about the mean as an estimate of the amount by which the mean of the eight angles differs from the true atmospheric anterina temperature. 


\begin{tabular}{lllllllllll}
\hline $\begin{array}{c}\text { Date } \\
\text { (Sept 1987) }\end{array}$ & $\begin{array}{c}\text { Time }(\mathrm{UT}) \\
(\mathrm{mK})\end{array}$ & $\begin{array}{c}+47^{\circ} \\
(\mathrm{mK})\end{array}$ & $\begin{array}{c}+40^{\circ} \\
(\mathrm{mK})\end{array}$ & $\begin{array}{c}+30^{\circ} \\
(\mathrm{mK})\end{array}$ & $\begin{array}{c}-30^{\circ} \\
(\mathrm{mK})\end{array}$ & $\begin{array}{c}-40^{\circ} \\
(\mathrm{mK})\end{array}$ & $\begin{array}{c}-47^{\circ} \\
(\mathrm{mK})\end{array}$ & $\begin{array}{c}-54^{\circ} \\
(\mathrm{mk})\end{array}$ & $\begin{array}{c}\text { Mean Atm } \\
(\mathrm{mK})\end{array}$ \\
\hline 9 & $7: 21-8: 48$ & 1307 & 1240 & 1191 & 1190 & 1090 & 1139 & 1174 & 1216 & $1193(38)$ \\
10 & $9: 45-11: 00$ & 1246 & 1183 & 1117 & 1125 & 1080 & 1082 & 1125 & 1178 & $1142(38)$ \\
11 & $2: 12-2: 54$ & 1314 & 1235 & 1173 & 1232 & 1017 & 1089 & 1152 & 1215 & $1178(28)$ \\
& $9: 30-10: 23$ & 1253 & 1181 & 1124 & 1101 & 1072 & 1114 & 1135 & 1182 & $1145(25)$ \\
12 & $7: 10-8: 40$ & 1429 & 1297 & 1222 & 1153 & 1102 & 1162 & 1246 & 1329 & $1242(80)$ \\
13 & $4: 37-6: 03$ & 1310 & 1188 & 1110 & 1085 & 923 & 1006 & 1107 & 1233 & $1120(38)$ \\
14 & $5: 34-6: 23$ & 1380 & 1273 & 1203 & 1190 & 1182 & 1151 & 1204 & 1304 & $1236(24)$ \\
& $6: 24-6: 51$ & 1272 & 1187 & 1156 & 1152 & 1106 & 1107 & 1140 & 1206 & $1166(39)$ \\
& $12: 15-13: 27$ & 1289 & 1174 & 1069 & 973 & 956 & 1035 & 1098 & 1174 & $1096(44)$ \\
15 & $3: 57-4: 41$ & 1371 & 1261 & 1180 & 1125 & 1192 & 1239 & 1273 & 1340 & $1248(22)$ \\
& $4: 42-5: 39$ & 1238 & 1168 & 1101 & 1028 & 1171 & 1247 & 1259 & 1272 & $1185(20)$ \\
& $9: 38-10: 50$ & 1214 & 1166 & 1103 & 1082 & 1163 & 1159 & 1195 & 1222 & $1163(26)$ \\
& $10: 55-14: 53$ & 1253 & 1163 & 1060 & 995 & 1098 & 1107 & 1165 & 1218 & $1133(52)$ \\
17 & $7: 36-10: 10$ & 1227 & 1178 & 1146 & 1145 & 1110 & 1078 & 1156 & 1208 & $1156(48)$ \\
& $11: 42-13: 12$ & 1209 & 1158 & 1092 & 1062 & 1072 & 1073 & 1135 & 1184 & $1123(30)$ \\
18 & $6: 09-11: 33$ & 1185 & 1117 & 1045 & 992 & 1140 & 1124 & 1149 & 1201 & $1119(30)$ \\
19 & $2: 18-8: 38$ & 1231 & 1161 & 1097 & 1048 & 1137 & 1132 & 1193 & 1262 & $1158(37)$ \\
20 & $3: 35-11: 30$ & 1184 & 1118 & 1038 & 1011 & 1099 & 1119 & 1140 & 1209 & $1115(51)$ \\
21 & $1: 03-14: 16$ & 1198 & 1118 & 1059 & 990 & 1233 & 1176 & 1207 & 1266 & $1156(43)$ \\
\hline
\end{tabular}

Table 3.5: Summary of atmospheric results for secondary atmospheric scans, September 1987 UT. $+54^{\circ}$ to $-54^{\circ}$ are the calculated values for $T_{A, A t m}$ at that angle, averaged over the time span of the observation. "Mean Atrn" refers to the mean of the eight scan angles of the given observation. The number in parentheses following "Mean Atm" is the RMS scatter of the time series of mean values within the given observation, and is an estimate of the noise of the measurement.

\begin{tabular}{llcc}
\hline Date & Time (UT) & Primary $\mathrm{T}_{\text {A.Am }}$ & Secondary $\mathrm{T}_{\text {A,Amm }}$ \\
\hline 14 Sept & $5: 34-6: 23$ & --- & $1236 \pm 11$ \\
& $6: 24-6: 51$ & -- & $1166 \pm 20$ \\
& $9: 59-11: 04$ & $1136 \pm 22$ & {$[137 \pm 25]$} \\
& $12: 15-13: 27$ & --- & $1096 \pm 13$ \\
15 Sept & $3: 57-4: 41$ & & $1248 \pm 8$ \\
& $4: 42-5: 39$ & -- & $1185 \pm 6$ \\
& $8: 02-8: 58$ & $1150 \pm 13$ & {$[1174 \pm 9]$} \\
$9: 38-10: 50$ & --- & $1163 \pm 8$ \\
$10: 55-14: 53$ & -- & $1133 \pm 8$ \\
\hline
\end{tabular}

Table 3.6: Comparison of primary and secondary atmospheric scans. The quoted uncertainties are $68 \%$ confidence level estimates of the statistical uncertainty only. The values in brackets are interpolations assuming a linear drift throughout the night. 
Evidence that the mean of all eight scan angles is a valid estimator of $T_{A, A t m}$ lies in additional measurements of $T_{A, A m}$ taken with the primary antenna during measurements of $\mathrm{T}_{\mathrm{A}, \text { zenich }}$ over the cold load. As a precaution, we measured $\mathrm{T}_{\mathrm{A}, \mathrm{A} \text { tm }}$ at $\pm 40^{\circ}$ during the actual CMB measurements on Sept 14 and 15. A comparison of the values for $T_{A, A t m}$ obtained from the primary and secondary systems is shown in Table 3.6. The primary and secondary systems have radically different systematics, but agree quite closely in the calculated $\mathrm{T}_{\mathrm{A}, \mathrm{Atm}}$.

Adding the $71 \mathrm{mK}$ estimate of systematics of unknown origin in quadrature with the $41 \mathrm{mK}$ limit on known systematics (Table 3.4) yields an estimate for the total systematic uncertainty in $T_{A, A t m}$ of $82 \mathrm{mK}$. To this must be added the estimate of statistical uncertainty in the mean from random fluctuations in the signal (typically $12 \mathrm{mK}$ ).

\subsubsection{Galactic Profile}

During the nights of Sept. 17-21, the radiometer performed long-duration atmospheric scans in an E-W orientation. These data can be used to extract the differential galactic signal, which we compared to the signal predicted by our galactic model. For each scan (consisting of observations of the eight atmospheric angles and the zenith), we took the differential signals for each of the four angle pairs

$$
\begin{aligned}
\Delta \mathrm{T}_{\mathrm{Galaxy}} & =\mathrm{G}\left[\mathrm{S}\left(\theta_{\mathrm{E}}\right)-\mathrm{S}\left(\theta_{\mathrm{w}}\right)\right] \\
& =\mathrm{B}(\alpha(\mathrm{t}), \delta)-\mathrm{B}\left(\alpha\left(\mathrm{t}-\mathrm{t}_{\theta}\right), \delta\right)
\end{aligned}
$$

where $S\left(\theta_{E}\right)$ and $S\left(\theta_{W}\right)$ are the signals of the east and west beams at zenith angle $\theta, B$ is the antenna temperature of the galaxy at celestial coordinates $(\alpha, \delta)$, and to is the time delay between the two angles. The declinations and time delays from each of the 4 pairs of angles are summarized in Table 3.7. A scan of the four angle pairs took approximately 6 minutes; to improve the statistics, we have binned the measurements of $\Delta \mathrm{T}_{\mathrm{Galaxy}}$ into $4^{\circ}$ bins in right ascension, using the R.A. of the positive (east) lobe.

The differential galactic profiles from Sept 17-21 are shown in Figure 3.4, along with the signal predicted by our galactic model. The data are noisy, as the atmospheric system was not designed for galactic scans (the time delays are long), but are consistent with the expected signal and show no evidence that the differential galactic signals subtracted from atmospheric temperature difference $\Delta T_{\mathrm{Atm}}$ are grossly in error. 
$\pm 30^{*}$ Galnctic Difierence

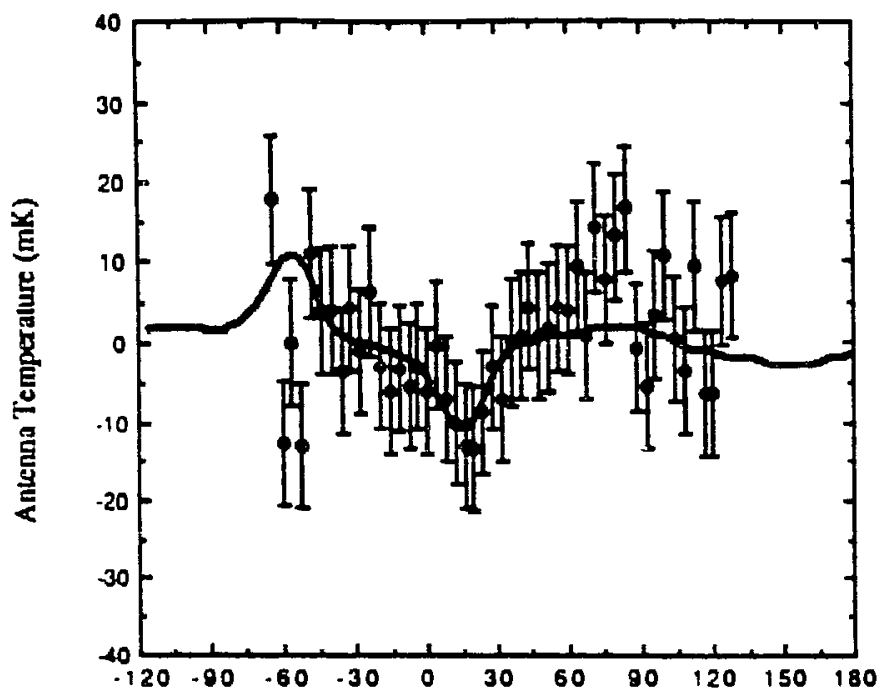

Right Ascension (degres)

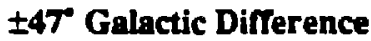

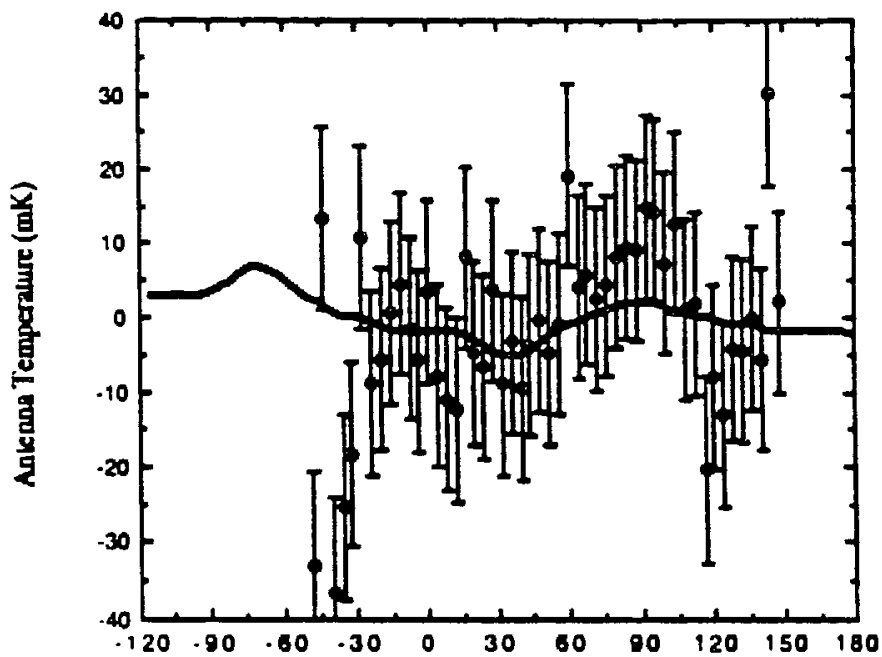

Right Accension (degrees) $\pm 40^{\circ}$ Galactiء Difference

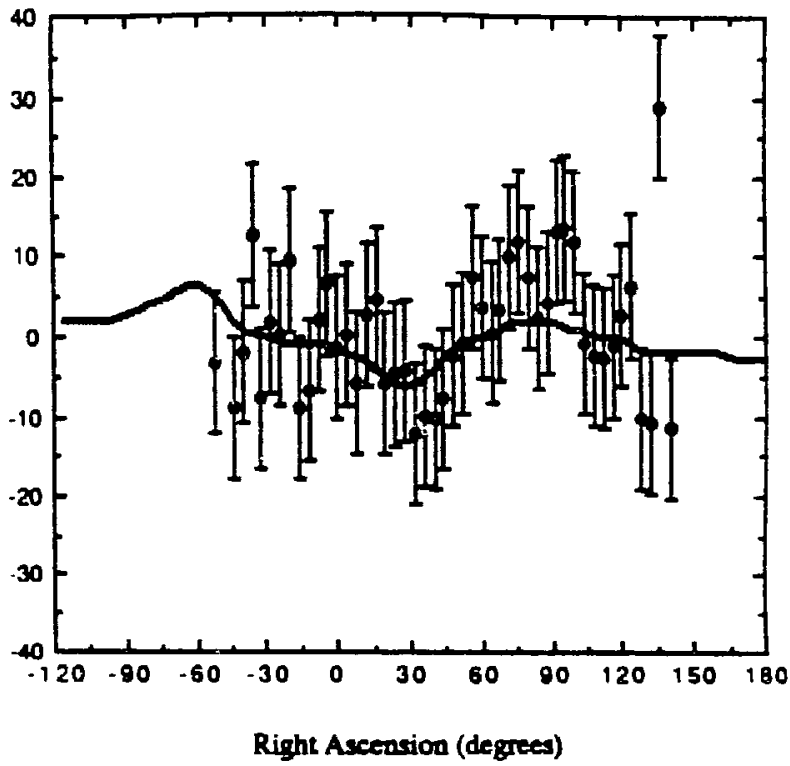

$\pm 4^{\circ}$ Galactic Difference

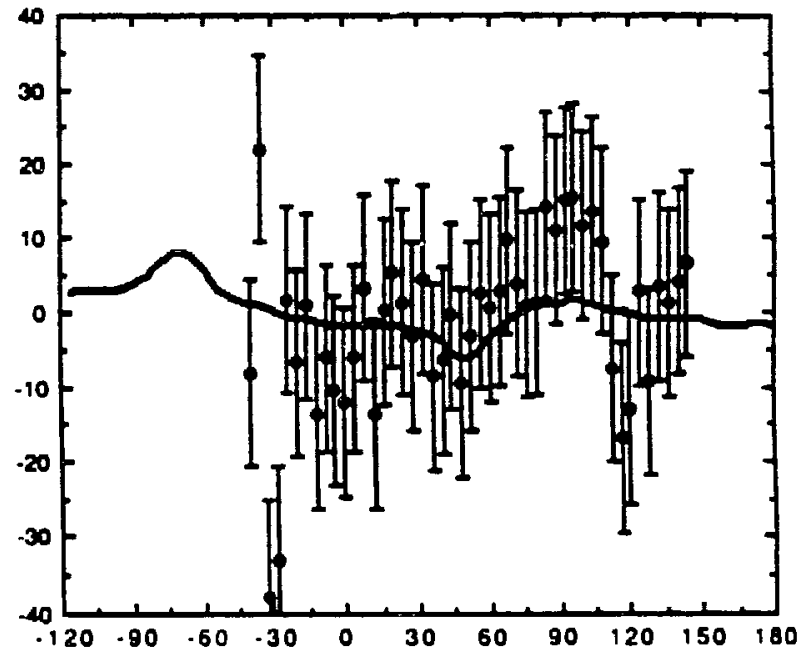

Right Ascension (degrees)

Figure 3.4: Results of differential galactic scans at $10 \mathrm{GHz}$ (points), along with the signal predicted from extrapolations of maps at lower frequencies. Typical error bars are shown. 


\subsubsection{Zenith Sky Temperature}

The radiometer measured $T_{A, z e n i t h}$ over the cold load on the nights of Sept 14 and 15, 1987 (UT). A strong frontal system rolled through the White Mountains several hours before the measurement on Sept 14, bringing clouds and a brief hailstorm. We have excluded any atmospheric data taken during this period. The aftermath of the front brought clear weather and a large drift in atmospheric temperature, which grew progressively colder at $18 \mathrm{mK} / \mathrm{hr}$ throughout the rest of the night. The barometric pressure was $489 \pm 1 \mathrm{~mm}$ $\mathrm{Hg}$, corresponding to a cold load reference temperature $\mathrm{T}_{\mathrm{A}, \mathrm{load}}=3.568 \pm 0.013 \mathrm{~K}$. The radiometer made 18 measurements on Sept 14 and an additional 25 on Sept 15. Adding the statistical uncertainty in quadrature with the systematic uncertainty (Table 3.4) yielded the following values for the zenith sky:

$$
\begin{aligned}
& \mathrm{T}_{\text {A,zenith }}=3.468 \pm 0.030 \mathrm{~K}(\text { Sept } 14) \\
& \mathrm{T}_{\mathrm{A} \text {,zenith }}=3.605 \pm 0.028 \mathrm{~K}(\text { Sept } 15)
\end{aligned}
$$

Only part of the difference between the two results is explained by atmospheric variation. $T_{A, A t m}$ is $0.037 \mathrm{~K}$ warmer during the measurement of the Sept 15 , as interpolated from secondary atmospheric scans, leaving a discrepancy of $0.1 \mathrm{~K}$ between the two measurements of $\mathrm{T}_{\mathrm{A}, \mathrm{zenith}}$.

\subsubsection{CMB Temperature}

We have calculated the antenna temperature of the CMB using Eq. 2.7 for each of the 43 measurements of $T_{A, z e n i t h}$. We used a value for $T_{A, A t m}$ for each individual scan obtained by fitting a linear atmospheric drift to the secondary atmospheric data of each night. The correction for Galactic emission was small $(6 \pm 3 \mathrm{mK})$. The resultant 43 determinations of $\mathrm{T}_{\mathrm{A}, \mathrm{CMB}}$ have a mean of $2.387 \mathrm{~K}$ and a RMS of $0.084 \mathrm{~K}$, shown in Figure 3.5. The distribution approximates a Gaussian distribution; we thus take the statistical uncertainty in the mean of the parent distribution to be $0.013 \mathrm{~K}$. Table 3.8 shows the error budget for $T_{A, C M B}$.

Converting the value for $T_{A, C M B}$ from antenna temperature to thermodynamic (brightness) temperature yields the value for $\mathrm{T}_{\mathrm{CMB}}$ :

$$
\left.\mathrm{T}_{\mathrm{CMB}}=2.62 \pm 0.09 \mathrm{~K} \text { (1987 result }\right)
$$




\begin{tabular}{ccc}
\hline Angle Pair & Declination (1950.0) & Time delay (hours) \\
\hline $\pm 30^{\circ}$ & $32^{\circ}$ & 4.8 \\
$\pm 40^{\circ}$ & $28^{\circ}$ & 6.2 \\
$\pm 47^{\circ}$ & $25^{\circ}$ & 7.1 \\
$\pm 54^{\circ}$ & $21^{\circ}$ & 8.0 \\
\hline
\end{tabular}

Table 3.7: Declination and time delay for differential galactic scans.

\begin{tabular}{lc}
\hline \multicolumn{1}{c}{ Source of Uncertainty } & Magnitude $(\mathrm{K})$ \\
\hline Statistical Uncertainty of $\mathrm{T}_{\mathrm{A} \text {, zenich }}$ & 0.013 \\
Systematic Uncertainty of $\mathrm{T}_{\mathrm{A} \text {, zenich }}$ & 0.025 \\
Statistical Uncertainty of $\mathrm{T}_{\mathrm{A}, \mathrm{Atm}}$ & 0.012 \\
Known Systematics in $\mathrm{T}_{\mathrm{A}, \mathrm{Atm}}$ & 0.041 \\
Systematic Atmospheric Offset & 0.073 \\
\hline \multicolumn{1}{c}{ Total: } & 0.089 \\
\hline
\end{tabular}

Table 3.8: Total error budget of $\mathrm{T}_{\mathrm{A}, \mathrm{CMB}}$. The term "Systematic Atmospheric Offset" is an estimate of the uncertainty in the mean value of $T_{A, A t m}$ caused by the unknown source of the observed atmospheric offsets.

\begin{tabular}{lll}
\hline \hline Year & $\mathrm{T}_{\mathrm{CMB}}$ & \multicolumn{1}{c}{ Comment } \\
\hline 1982 & $2.66 \pm 0.12$ & $\mathrm{~T}_{\mathrm{A}, \mathrm{Amm}}$ estimated from $90 \mathrm{GHz}$ results \\
1983 & $2.63 \pm 0.14$ & $\mathrm{~T}_{\mathrm{A}, \mathrm{Amm}}$ from primary scans \\
1984 & $2.65 \pm 0.21$ & Primary scans, stationary elliptical mirror \\
1986 & $2.56 \pm 0.08$ & $\mathrm{~T}_{\mathrm{A}, \mathrm{Amm}}$ from secondary scans \\
1987 & $2.62 \pm 0.09$ & $\mathrm{~T}_{\mathrm{A}, \mathrm{A} m \text { from secondary scans }}$ \\
\hline \hline
\end{tabular}

Table 3.9: Thermodynamic temperature of the $\mathrm{CMB}$ at $10 \mathrm{GHz}$. 


\subsection{Overall Results}

The value for $\mathrm{T}_{\mathrm{CMB}}$ obtained in 1987 is in excellent agreement with the value obtained in previous years. Table 3.9 lists the results of the $10 \mathrm{GHz}$ radiometer from 1982 through 1987. It is non-trivial to extract a single value and error estimate from this data set: the same radiometer performed measurements over five summers under various configurations. The data from 1983 best represent the "original" radiometer configuration: data were taken exclusively by the primary system, with a fixed rectangular mirror on the secondary. In 1984, the rectangular mirror was replaced by the smaller elliptical mirror, changing somewhat the systematics of the experiment. The radiometer in 1986 and 1987 used the secondary scanning system to measure the atmosphere, changing the systematics yet again. Finally, the atmosphere in 1982 has been determined from concurrent measurements at 90 and $10 \mathrm{GHz}$ as discussed in Appendix A. Systematics dominate the error budget, yet it is not clear what fraction of the systematics are truly independent from year to year. I have chosen to evaluate the combined data set as follows:

The systematics for 1986 and 1987 are clearly very similar, and different from previous years. I use the mean of the 1986-87 data, with the 1987 error estimate, to represent the data taken with a working secondary system. The data from 1983 form a second independent data set. The data from 1982 and 1984 are independent of each other, but partially related to the 1983 and 1986 results. As the error estimates for 1984 and 1982 do not dominate a weighted average, I have chosen to regard them as fully independent. Using the 1982-84 data and the mean of the 1986-87 data, weighted by their error estimates, gives to final estimate of the temperature of the $\mathrm{CMB}$ at $10 \mathrm{GHz}$ :

$\mathrm{TCMB}=2.62 \pm 0.06 \mathrm{~K}$ (combined result) 


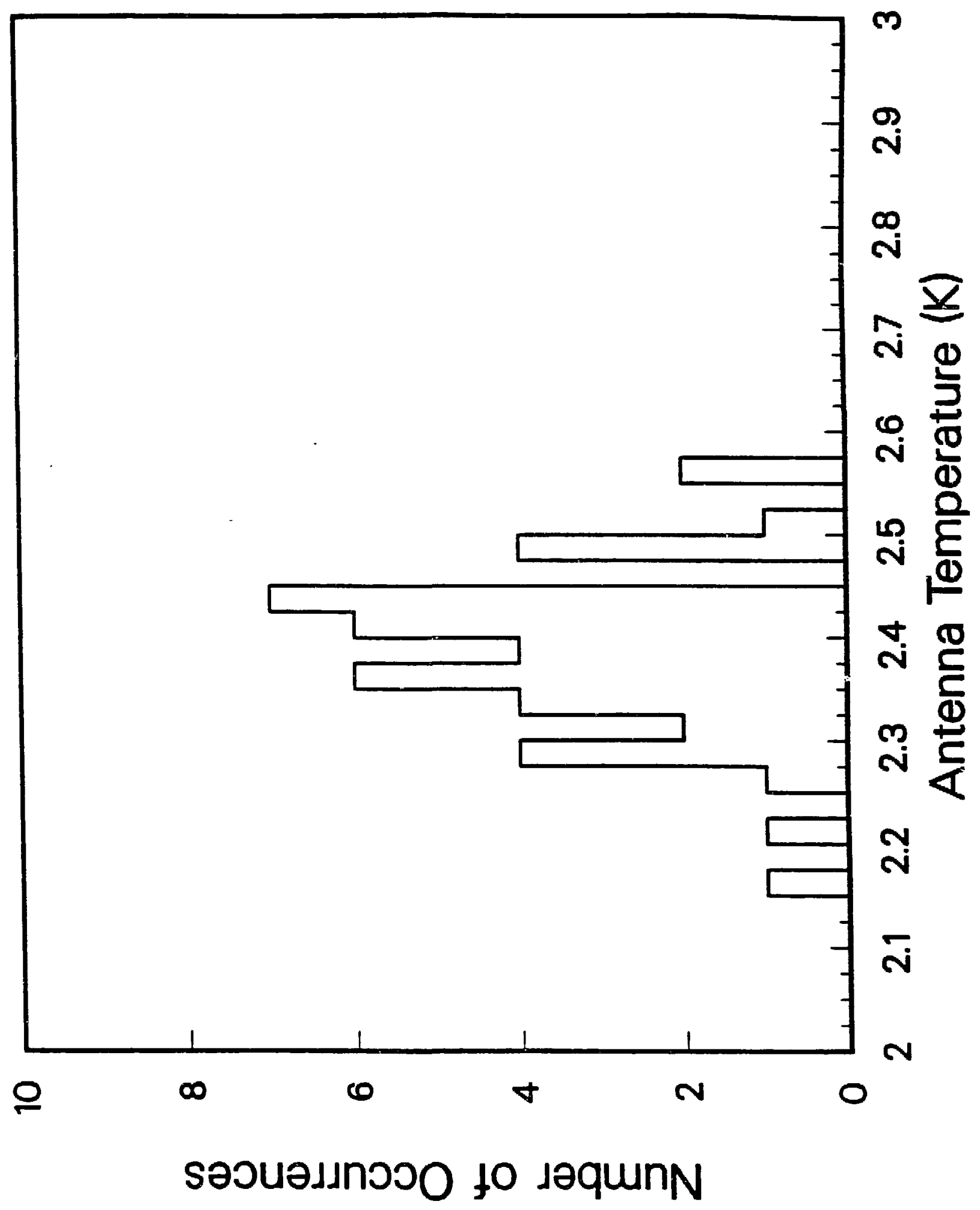

Figure 3.5: Histogram of 43 measurements of $T_{A, C M B}$ in 1987. 


\section{Chapter 4 \\ Measurement at $7.5 \mathrm{GHz}$}

\subsection{Introduction}

By early 1988, new pressure arose for precise low-frequency CMB measurements. The apparent sub-mm excess observed by Matsumoto et al. (1988) rapidly engendered a cottage industry of theoretical activity. A number of the models could be differentiated only by their behavior at low frequencies. At the same time, hints appeared that the lowfrequency measurements from White Mountain were systematically lower than the world average (excluding the sub-mm excess). In particular, we were concerned about the discrepancy between the $10 \mathrm{GHz}$ measurement,

$$
\mathrm{T}_{\mathrm{CMB}}(10 \mathrm{GHz})=2.62 \pm 0.06 \mathrm{~K} \text {, }
$$

and the results at $24 \mathrm{GHz}$ of Johnson and Wilkinson (1986),

$$
\mathrm{T}_{\mathrm{CMB}}(24 \mathrm{GHz})=2.783 \pm 0.025 \mathrm{~K} \text {. }
$$

To better characterize the low-frequency spectrum of the CMB, we embarked on a major effort to reduce the systematics of our ground-based measurements. We built a new cryogenic reference load (described below) optimized for use in the frequency range 1-10 $\mathrm{GHz}$ as opposed to the $2.5-90 \mathrm{GHz}$ range of the old cold load. We rebuilt the radiometer operating at $1.5 \mathrm{GHz}$, and added a new instrument at $7.5 \mathrm{GHz}$. Finally, we would observe from a near-ideal environment: near Amundsen-Scott station at the South Pole, where RFI and atmospheric temperature and variation reach minimal levels. The measurement described in this chapter is the result of a full-scale test of the system, performed from Barcroft in September 1988. We anticipate measurements at $1.5,3.8$, and $7.5 \mathrm{GHz}$, as well as atmospheric measurements at $90 \mathrm{GHz}$, using the new system at the South Pole in November 1989.

\subsection{Experimental Design}

\subsubsection{The Radiometer}

We had several goals in mind when we designed the radiometer. First and foremost, we wanted a measurement of the $\mathrm{CMB}$ temperature accurate to $0.05 \mathrm{~K}$. The atmosphere would still be the largest foreground signal; however, the $10 \mathrm{GHz}$ scanning mirror had led us to conclude that such a system at $7.5 \mathrm{GHz}$ would have systematics of the same order as a simple tip-scan. Instead, we would concentrate on removing the "flip offset" associated 


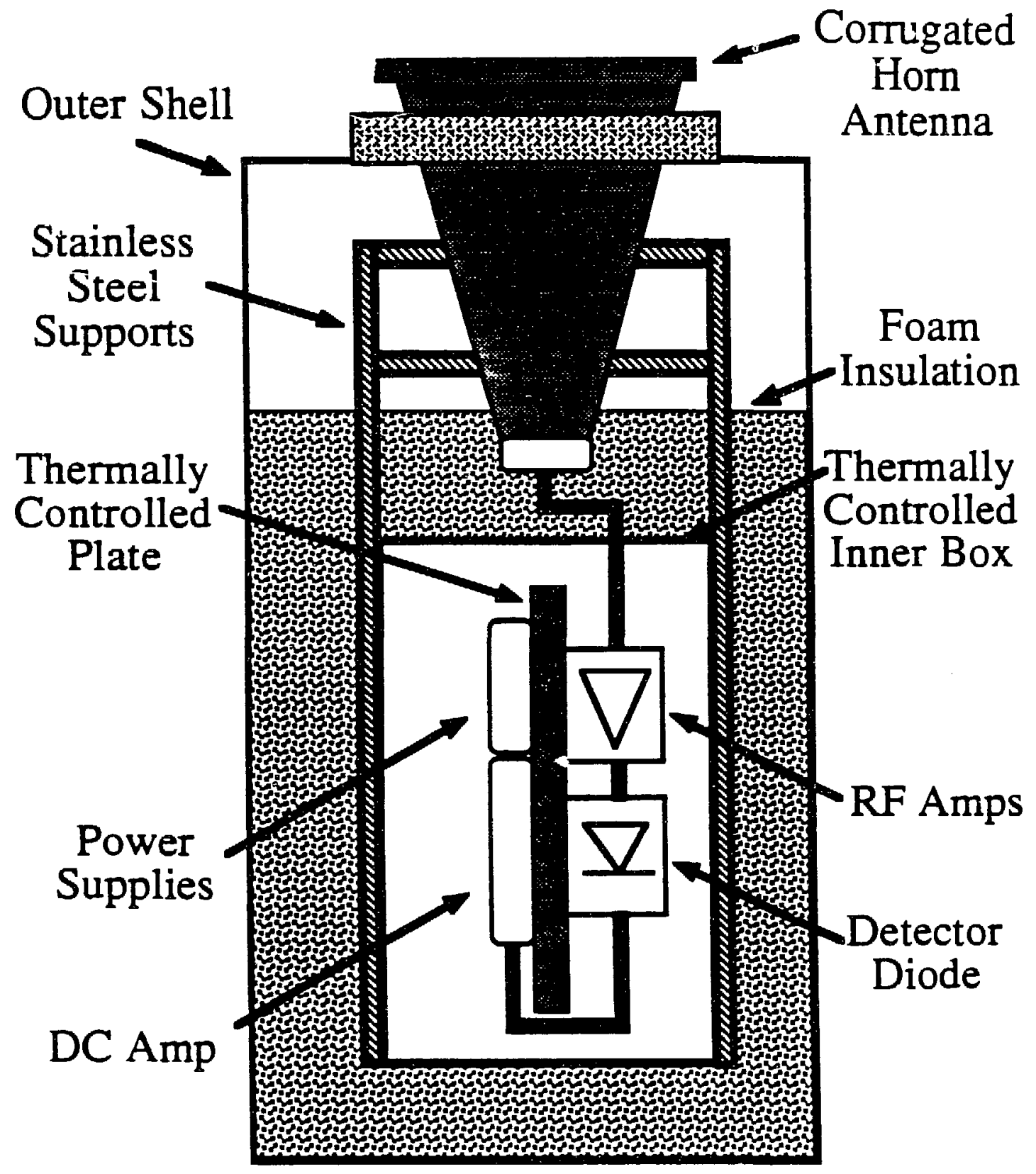

Figure 4.1: Schematic of the $7.5 \mathrm{GHz}$ radiometer. 
with moving the radiometer. We approached this goal with the concept of simplifying the design wherever possible.

The simplest radiometer is a total-power, direct-gain system. Our previous experience with this type of radiometer (De Amici et al. 1988, Levin er al. 1988) indicated that existing amplifier technology would allow a radiometer of sufficient short-term stability to be built and operated, provided the chopping frequency between two targets was above the knee of the $1 / f$ noise typical of such systems.

Drifts in RF amplifiers are dominated by thermal changes. The radiometer had to have extremely good thermal stability, and would need to operate in the harsh Antarctic environment with minimal power consumption. To shield the RF chain from the varying outside world, and to allow a $55 \mathrm{~K}$ gradient to exist between the amplifiers and the ambient temperature, we adopted a double-buffered design (Figure 4.1). We mounted the RF amplifiers and associated electronics on a $1-\mathrm{cm}$ thick aluminum plate, which was loosely heat sunk to an RF-tight aluminum box. Both the inner plate and the outer "thermal" box were temperature-controlled using independent differential heating circuits, with a nominal $25 \mathrm{~K}$ difference between the two to allow dissipation of waste heat from the RF components. In turn, we rigidly attached the thermal box to the antenna with stainless-steel struts. A variable amount of foam insulation could be placed between the thermal box and the outer shell of the radiometer, which would remain at ambient temperature. The thermal box would provide coarse thermal control, regulating the varying heat leak from the surroundings. The temperature gradient between the thermal box and the inner plate would then be fairly constant in time, with the thermal controller on the inner plate providing the remaining control required to keep the amplifiers' temperature constant to within $0.1 \mathrm{~K}$. With a typical gain temperature coefficient of $2 \% \mathrm{~K}^{-1}$, we would thus maintain gain stability within $2 \times 10^{-3}$ on time scales of several hours, with correspondingly better performance on the shorter (one minute) time scale of a single measurement.

Figure 4.2 shows the RF chain. The antenna was the same corrugated conical antenna used for the $10 \mathrm{GHz}$ experiment, with a new circular-to-rectangular waveguide transition optimized for $7.5 \mathrm{GHz}$. A short rectangular waveguide ended in a transition to rigid stainless-steel SMA coaxial cable, which was the only direct thermal link to the inner plate from an ambient-temperature sink. The signal from the input underwent $40 \mathrm{~dB}$ of $R F$ amplification in the first stage, a Miteq AMF-4S-7278-15 amplifier with a nominal 7.25$7.75 \mathrm{GHz}$ bandpass and an isolator on the input. The isolator on the first stage prevented load-dependent offsets. A second RF amplifier provided an additional $25 \mathrm{~dB}$ of gain. A 


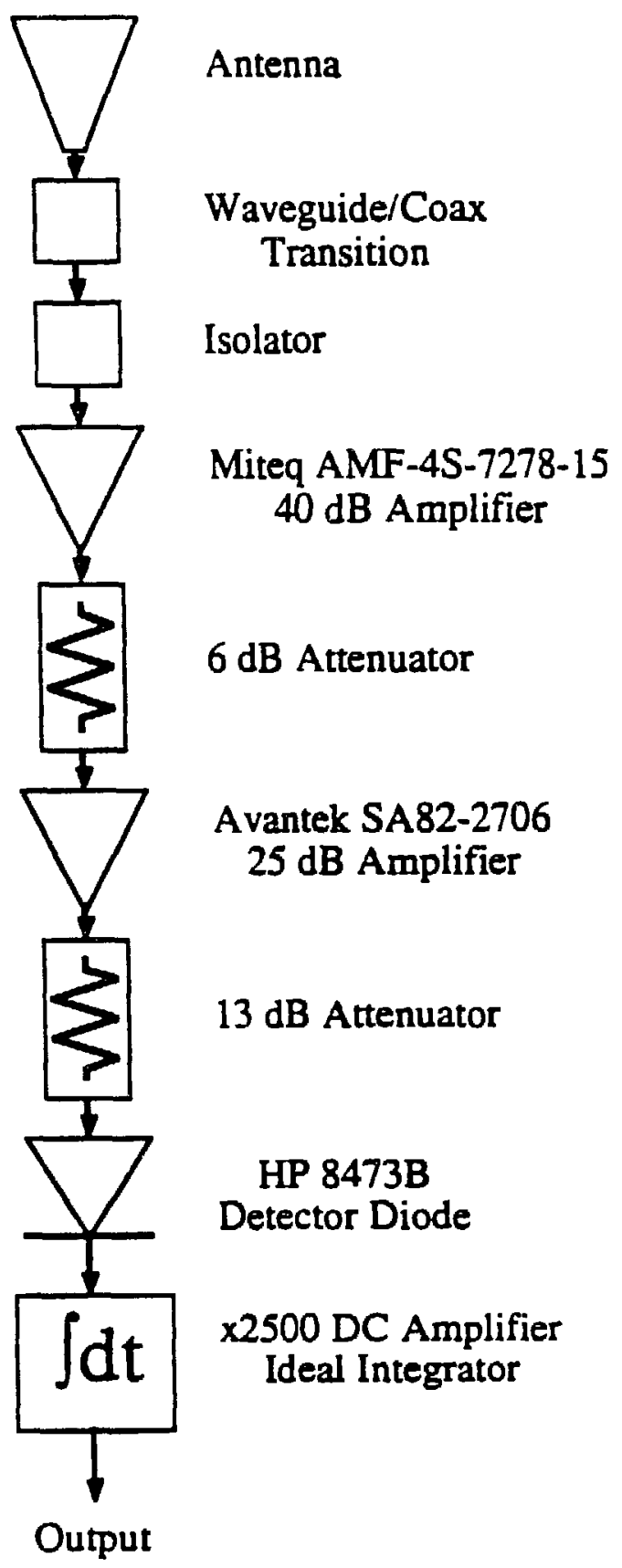

Figure 4.2: Schematic of the RF chain. 
Schottky-barrier HP 8473B diode rectified the amplified RF signa'. Attenuation of $6 \mathrm{~dB}$ between the two amplifiers and $13 \mathrm{~dB}$ between the second amplifier and the detector diode served to reduce reflections among the RF components. The fairly low RF amplification ensured operation of the diode in its linear regime. The signal from the diode underwent an additional amplification of 2500 in a custom-built DC amplifier, which integrated the signal for a period of 2 seconds as determined by an external $100 \mathrm{~Hz}$ clock signal. This unit contained its own thermal controller and insulation, and was also mounted on the inner plate. We measured the system temperature of the radiometer at Barcroft to be $234 \pm 1 \mathrm{~K}$.

The amplified DC signal was digitized and recorded by the same multiplexer/ADC used by the $10 \mathrm{GHz}$ radiometer in previous years. The $7.5 \mathrm{GHz}$ radiometer shared the system in 1988 with radiometers operating at frequencies of $1.5,3.8$, and $90 \mathrm{GHz}$. A cassette recorder and digital computer recorded the radiometer output every 2 seconds (the integration period). In addition to the output voltage, the system recorded the temperatures of the inner RF plate, the thermal box, the antenna, and the ambient target, as well as the current drawn by the heater on the RF plate. The current drawn by the heater on the thermal box could be monitored but was not recorded. The output of an electronic clinometer recorded the zenith angle of the radiometer to a precision of \pm 6 '.

The coaxial cable between the antenna and the first amplifier is an obvious site for position-dependent changes in reflection, and hence a potential source of systematic offset changes. Four stainless-steel struts provided mechanical support between the inner RF plate and the antenna. Aluminum supports connected the outer shell of the radiometer to the antenna aperture but not to the RF plate, minimizing microphonic effects. Thin aluminum squares covered the outer shell to provide protection from the environment and the occasional clumsy operator. The total mass of the radiometer when assembled was approximately $20 \mathrm{~kg}$.

We measured the beam pattern of the antenna to have a FWHM of $20 \pm 2^{\circ}$ at $7.5 \mathrm{GHz}$. The calculated contribution from ground emission with this beam pattern was too large (100 mK) to model directly with the required precision. We built a large pyramidal ground shield that attached to the radiometer during atmospheric scans and the measurement of

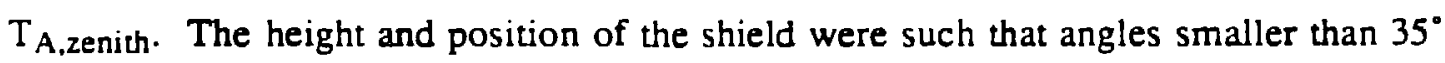
viewed the sky directly, while larger beam angles reflected from the shield. A flared section with flare radius of two wavelengths reduced diffraction over the edges of the shield. The far-field beam pattern of the corrugated horn antenna is shown in Figure 4.3, both with and without the ground shield normally in place for all observations of the sky. 


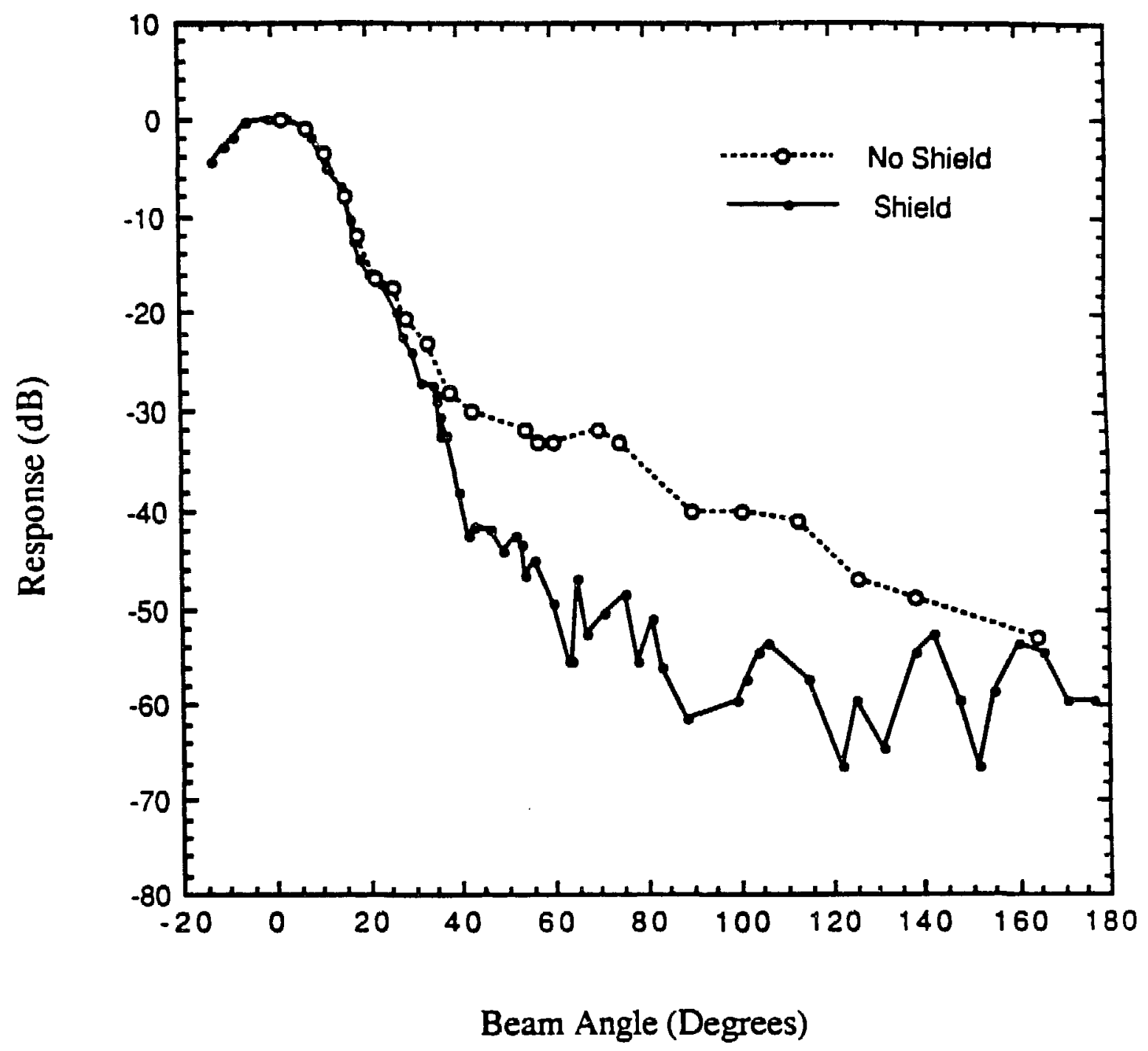

Figure 4.3: Measured far-field beam patterns at $7.5 \mathrm{GHz}$. 
The contribution of the ground shield can be seen for beam angles $>35^{\circ}$, beyond which the shield added between 10 and $20 \mathrm{~dB}$ additional rejection of the ground signal.

The ambient target used for calibration consisted of a slab of microwave absorber (Eccosorb CV-3) $25 \mathrm{~cm} \times 25 \mathrm{~cm} \times 8 \mathrm{~cm}$, enclosed within a metal box insulated by a $2.5 \mathrm{~cm}$ thick layer of closed-cell foam. The target could be opened to cover the antenna aperture completely, and was otherwise left closed to allow the interior to thermalize. A thermistor within the Eccosorb monitored the target temperature.

\subsubsection{The Reference Load}

We built a new cryogenic reference load in 1988, optimized for use between 1 and 10 $\mathrm{GHz}$. It differed from the previous reference load in several important aspects. The most important change for measurements at $7.5 \mathrm{GHz}$ was the method used to block the infrared heat leak to the absorber. The old cold load used a high-reflectance metal shutter below the warm polyethylene windows to reduce the IR load to the dewar. This allowed the radiometers to view the target through a minimum of material (two $25 \mu \mathrm{m}$ polyethylene windows), but was cumbersome and caused a large heat leak when the shutter was opened during observations of the target. In place of the shutter, the new cold load used two thin (76 and $175 \mu \mathrm{m}$ ) teflon-impregnated glass cloth (Fluorglas 381-3) windows. The windows had large absorption in the $\mathbb{R}$, but were essentially transparent below $10 \mathrm{GHz}$ (opacity $\tau \leq 0.0002$ at $7.5 \mathrm{GHz}$ ). Located in the radiometric space between the absorber and the upper windows, the Fluorglas windows intercepted IR radiation and warmed the LHe boiloff gas (Figure 4.4). The total heat leak to the new cold load was $-4 \mathrm{~W}$, compared to $-35 \mathrm{~W}$ with the old load. The reduced heat leak minimized variations in boiloff rate and allowed more and longer observations. The supply of cryogen was not a limiting factor in 1988.

The new cold load incorporated several other changes. The new microwave absorber (Emerson \& Cummings EHP-12 backed by E\&C LS) was thicker and optimized for frequencies as low as $1 \mathrm{GHz}$. The new cold load was somewhat wider at $78 \mathrm{~cm}$ diameter (radiometric wall), compared to the $70 \mathrm{~cm}$ of the old cold load. The transition from the top flange to the aluminized fiberglass radiometric wall was simplified and made smoother. with no steps or gaps larger than $0.5 \mathrm{~mm}$. A series of sensors behind the radiometric wall 


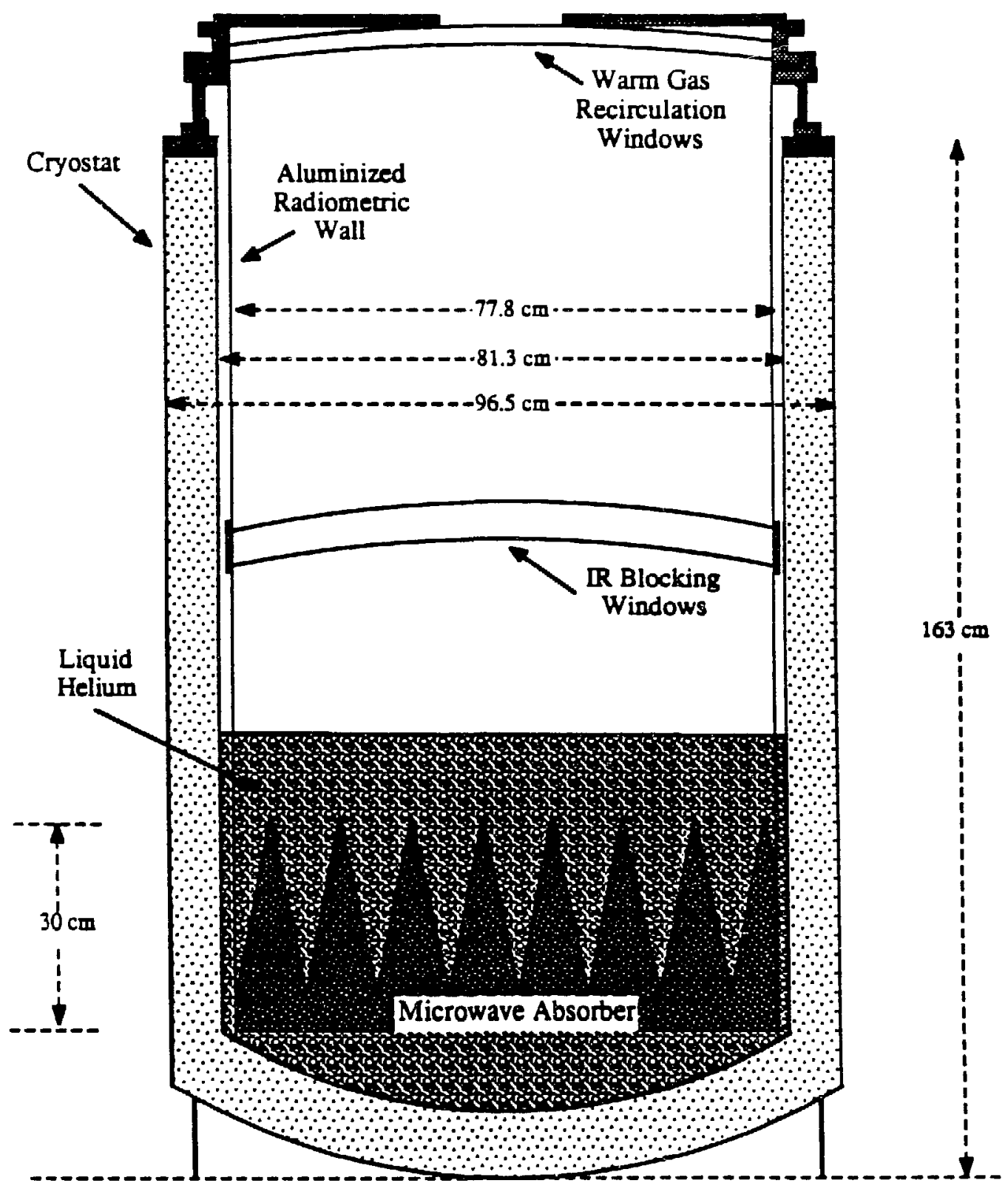

Figure 4.4: Schematic of the cold load used in 1988. 
allowed better estimation of cryogen level and the temperature profile in the radiometric wall.

We have extensively modelled and tested the radiometric properties of the cold load (Appendix B). The radiometer-cold load system may be described as a perfect absorber and a perfect radiometer, with a series of reflecting and emitting surfaces between them. For our purposes, the major terms are the emissivity and power reflection coefficients of the absorber, the glass/teflon IR-blocking windows, and the polyethylene windows; the power reflection coefficient of the radiometer; the power reflection coefficient of the helium liquid/gas interface; the temperatures of the absorber, glass/teflon windows, and polyethylene windows; and the broadcast temperature of the radiometer.

These terms are summarized in Table 4.1. For purposes of radiation broadcast by the radiometer and reflected back into the beam, one must also consider the illumination of the reflecting surface. The $18 \mathrm{~cm}$ diameter antenna aperture intercepts a fraction $\Gamma$ of the reflected beam, which can be calculated based on the beam width, geometry, and an assumption of specular reflection.

To lowest order, the antenna temperature of the reference target is simply the thermodynamic temperature of the cryogen, $\mathrm{T}_{\mathrm{Abs}}$, converted to antenna temperature. Given knowledge of the positions, reflectivity, and emissivity of the material between the radiometer and the absorber, contributions to $T_{A, l o a d}$ arising from reflected and emitted radiation can be calculated. At $7.5 \mathrm{GHz}$, these corrections are all small $(<20 \mathrm{mK})$. The largest single correction is the power reflected from the teflon/glass IR-blocking windows, estimated at $6 \mathrm{mK}$ for the two windows. The largest corrections for emission from sources other than the absorber are estimated at $6 \mathrm{mK}$ for emission from the teflon/glass windows and $6 \mathrm{mK}$ emission from joinis in the radiometric wall. Other sources of emission or reflection are smaller still.

Radiometer broadcast power, reflected from the windows, can interfere coherently and give rise to reflection arms proportional to the sum of the amplitude reflection coefficients of the interfering smires. The only surfaces with significant reflectivity within the $60 \mathrm{~cm}$ coherence length of th: radiometer are the teflon/glass windows. The estimated magnitude of the coherently interfering term frcm the windows is $<0.001 \mathrm{~K}$. We tested for this effect by raising the radiometer in a specially-constructed device over the cold load, effectively changing the path length between the radiometer and any reflecting surfaces within the cold load. We observed no signal within the $0.02 \mathrm{~K}$ noise level of the test. 


\begin{tabular}{lcccc}
\hline & $\begin{array}{c}\text { Power Reflection } \\
\text { Coefficient }\end{array}$ & Emissivity & $\begin{array}{c}\text { Reflected } \\
\text { nlumination }\end{array}$ & $\begin{array}{c}\text { Temperature } \\
(\mathrm{K})\end{array}$ \\
\hline Absorber & $<10^{-5}$ & 0.9999 & 0.02 & 3.771 \\
Polyethylene & $5 \times 10^{-6}$ & $2 \times 10^{-6}$ & 1.0 & 270 \\
Upper IR Window & $4 \times 10^{-5}$ & $5 \times 10^{-5}$ & 0.08 & 50 \\
Lower IR Window & $2 \times 10^{-4}$ & $1 \times 10^{-4}$ & 0.08 & 30 \\
Helium Interface & $1.5 \times 10^{-4}$ & - & 0.02 & 3.771 \\
\hline Radiometer & $<0.01$ & $-\ldots$ & 1.0 & 300 \\
\hline \hline
\end{tabular}

Table 4.1: Properties of the reference target.

\begin{tabular}{lc}
\hline Term & Magnitude $(\mathrm{K})$ \\
\hline Absorber Emission & $3.594 \pm 0.002$ \\
Other Emission & $0.013 \pm 0.007$ \\
Power Reflection & $0.006 \pm 0.005$ \\
Coherent Reflection & $0 \pm 0.004$ \\
\hline TA,Load & $3.613 \pm 0.009 \mathrm{~K}$ \\
\hline \hline
\end{tabular}

Table 4.2: Contributions to $T_{A, l o a d}$.

\begin{tabular}{|c|c|c|c|c|c|c|c|}
\hline Nominal Angle & $-40^{\circ}$ & $-30^{\circ}$ & $-15^{\circ}$ & Zenith & $+15^{\circ}$ & $+30^{\circ}$ & $+40^{\circ}$ \\
\hline Mean Angle & $-40^{\circ} 19^{\prime}$ & $-30^{\circ} 22^{\prime}$ & $-15^{\circ} 29^{\prime}$ & $-0^{\circ} 42^{\prime}$ & $+14^{\circ} 31^{\prime}$ & $+29^{\circ} 43^{\prime}$ & $+40^{\circ} 4^{\prime}$ \\
\hline Single-run RMS & $1^{\prime}$ & 1 & $2^{\prime}$ & $2^{\prime}$ & 1 & $2^{\prime}$ & $2^{\prime}$ \\
\hline Total RMS & $8^{\prime}$ & $8^{\prime}$ & 3 & 2 & $2^{\prime}$ & $2^{\prime}$ & $6^{\prime}$ \\
\hline
\end{tabular}

Table 4.3: Measured pointing of the atmospheric scan cart. The single-run RMS is the scatter within a typical 30-minute observing run. The total RMS is the scatter over the entire stay at Barcroft. The cart was aligned E-W within $2^{\circ}$ with $(+)$ angles to the east. 
Emission from the various windows can be calculated from their emissivity and temperature. Temperature sensors behind the radiometric wall at the level of the teflon/glass windows recorded a temperature of $30 \mathrm{~K}$ for the lower window and $50 \mathrm{~K}$ for the upper window, which we take as the temperature of the respective windows. Each window contributes $3 \mathrm{mK}$ to $\mathrm{T}_{\mathrm{A} \text {,load. }}$ Emission from the He gas column is negligible. The aluminized radiometric wall has a small emissivity; convolved with the antenna beam pattern and the measured temperature profile of the wall, it contributes less than $0.001 \mathrm{~K}$ to $T_{A, l o a d}$.

The barometric pressure at Barcroft in 1988 was $481 \pm 1 \mathrm{~mm} \mathrm{Hg}$, somewhat lower than in previous years. This corresponds to a LHe boiling point of $3.771 \pm 0.002 \mathrm{~K}$ (Donnelly 1967). The contributions to $T_{\mathrm{A}, \text { load }}$ are summarized in Table 4.2. We took the antenna temperature of the reference target in 1988 to be

$$
\mathrm{T}_{\mathrm{A}, \mathrm{load}}=3.613 \pm 0.009 \mathrm{~K} \text {. }
$$

\subsection{Data Collection Routines}

\subsubsection{Zenith Sky Measurement}

The radiometer measured $T_{A, z e n i t h}$ by comparing the signals from the cold load and the zenith sky, observed in close succession. We used the following procedure:

- The radiometer observed the cold load for 32 seconds.

-We spent 16 seconds moving the radiometer from the down position on the cold load to the up position one meter from the cold load. We placed the ground shield over the antenna. Data from this time period were ignored.

- The radiometer spent 32 seconds observing the zenith sky with the ground shield in place.

- We spent 16 seconds removing the ground shield and placing the ambient target over the antenna. Data from this time were ignored.

- The radiometer observed the ambient target for 16 seconds. During this time, we checked the top polyethyiene window for dust or condensation, wiping it as necessary. 
- We spent 16 seconds moving the radiometer from the upright position to the down position over the cold load. Data from this period were ignored.

A complete scan sequence took 128 seconds, with only 16 seconds separating the observations of the cold load and the zenith sky. A typical observing run over the cold load lasted 40 minutes and comprised some 15 independent measurements of $T_{A, z e n i t h}$.

We measured $\mathrm{T}_{\mathrm{A}, \text { zenich }}$ with LHe in the cold load on 1988 Sept $165: 45-6: 15$ and 8:28-9:13; 1988 Sept 17 11:45-12:07; and 1988 Sept 19 10:13-10:47 UT, obtaining 65 independent measurements of $\mathrm{T}_{\mathrm{A}, \mathrm{zenith}}$. As a test of the scan procedure and equipment, we measured $\mathrm{T}_{\mathrm{A}, z e n i c h}$ with liquid nitrogen in the cold load on the night of 1988 Sept 14, 8:02-8:38 UT.

\subsubsection{Atmospheric Measurement}

We determined the atmospheric antenna temperature by comparing the signal from the zenith sky to the signal when the radiometer was tipped to angles of $30^{\circ}$ and $40^{\circ}$ to either side of the zenith. For this purpose, we used a small cart, aligned E-W so that observations on one side of the radiometer (the " $+"$ side) had azimuth $+90^{\circ}$ (due east) while observations on the other side ("-") had azimuth $-90^{\circ}$. The ground shield remained firmly attached to the radiometer throughout the entire measurement. The radiometer rested on its handles, which served as the axis of rotation. An aluminum bar, attached to the bottom of the radiometer shell, connected to a series of pins on an outrigger and held the radiometer at fixed zenith angles. We measured the pointing of the system daily with a precision bubble clinometer and found it to be quite repeatable. The RMS variation from one scan to the next was $1-2$ arc $\min$; between one day and the next, the pointing was stable to within $6^{\prime}$. Our analysis of atmospheric data used the results of each day's pointing measurements. Pointing information is summarized in Table 4.3.

We used the following procedure when measuring $T_{A, A t m}$ :

- The radiometer spent 32 seconds at each of the positions $-40^{\circ},-30^{\circ}$, zenith, $+30^{\circ}$, and $+40^{\circ}$.

- The radiometer moved from $+40^{\circ}$ directly to $-40^{\circ}$; we spent 16 seconds placing the ambient target over the antenna aperture. We did not remove the ground shield. Data from this period were ignored. 
- The radiometer observed the ambient target for 32 seconds.

- We spent 16 seconds removing the ambient target from the antenna. Data from this period were ignored. The radiometer remained in the $-40^{\circ}$ position throughout the calibration procedure.

A single atmospheric scan took 224 seconds, during which period we determined $\mathrm{T}_{\mathrm{A}, \mathrm{Atm}}$ from the four angles observed. The maximum elapsed time between measurements of the zenith and angle $\theta$ was 32 seconds. An atmospheric run typically lasted an hour and comprised 10 to 15 complete scans.

We used an alternate scanning procedure on the night of Sept 19 in an effort to concentrate the observing time on the eastem $(+)$ angles which had a much smaller correction for $T_{A, G r o u n d}$. We calibrated only once every other scan, and observed the eastern angles exclusively, using the following procedure:

- The radiometer spent 32 seconds at each of the positions zenith, $+30^{\circ}$, $+40^{\circ}$, zenith, $+30^{\circ}$, and $+40^{\circ}$.

- We moved the radiometer directly from $+40^{\circ}$ to $-40^{\circ}$ and spent 16 seconds placing the ambient calibration target over the antenna aperture. Data from this period were ignored.

- The radiometer observed the ambient target for 32 seconds.

- We spent 16 seconds removing the ambient target and tilting the radiometer to view the zenith again. Data from this period were ignored.

A single scan now took either 96 seconds or 160 seconds, depending on whether a calibration was included. A typical run lasted 90 minutes and comprised 20 to 30 independent scans. We measured $T_{A, A t m}$ on the nights of 1988 Sept 3, 4, 5, 7, 9, 11, 14, $15,16,17$, and $19 \mathrm{UT}$.

\subsubsection{Galactic Measurement}

We measured the differential galactic profile by comparing the signal when the radiometer pointed $15^{\circ}$ east of zenith to the signal when the radiometer pointed $15^{\circ}$ west of zenith. We used either the atmospheric cart, which included angles of $\pm 15^{\circ}$ (Table 4.3), or a small tumtable built for the purpose. The procedure varied slightly depending on the method used. The turntable used the following sequence: 
- The radiometer viewed the sky at $15^{\circ}$ east of zenith for 16 seconds.

- The turntable spent 16 seconds rotating $180^{\circ}$ to allow the radiometer to view the sky at $15^{\circ}$ west of zenith. For the latter half of this period, the radiometer was in the new position. We ignored data from the full 16 seconds to allow residual rocking or wobbling motion to damp out.

- The radiometer observed the sky at $15^{\circ}$ west of zenith for 16 seconds.

- The turntable spent 16 seconds rotating $180^{\circ}$ to allow the radiometer to view the sky at $15^{\circ}$ west of zenith. Again, we ignored data from the full 16 seconds.

- Approximately once per hour, we stopped the radiometer in the $15^{\circ}$ west position and allowed it to view the ambient calibration target for at least 32 seconds. Following calibration, we re-started the turntable and resumed scanning.

Scans on the atmospheric cart were similar, but did not require 16 seconds to change from one position to the other. We calibrated approximately once per hour in either case.

Both the atmospheric cart and the tumtable were aligned $\mathrm{E}-\mathrm{W}$ within $1^{\circ}$; as a result, the radiometer chopped between two positions at declination $36^{\circ}$ separated by 2.4 hours in right ascension. We measured the differential galartic profile on the nights of 1988 Sept 5 , 6,8 , and 10 UT. Over the course of these nights, we obtained coverage of the track from R.A. $=-60^{\circ}$ to R.A. $=+112^{\circ}$, observed simultaneously with all four radiometers at 1.5 , $3.8,7.5$, and $90 \mathrm{GHz}$.

\subsection{System Performance Tests}

\subsubsection{Temperature Stability}

The radiometer was designed to operate at an ambient temperature of $-40 \mathrm{C}$ while drawing minimal power. Temperatures at night at Barcroft ranged from +6 to $-10 \mathrm{C}$, a considerably hotter environment. To allow the radiometer to operate without overheating, we removed the insulation between the outer shell and the thermal box. In addition, we raised the operating temperature of the inner plate from the design goal of $+5 \mathrm{C}$ to $+29 \mathrm{C}$, the lowest value that allowed adequate temperature regulation of the inner plate. 


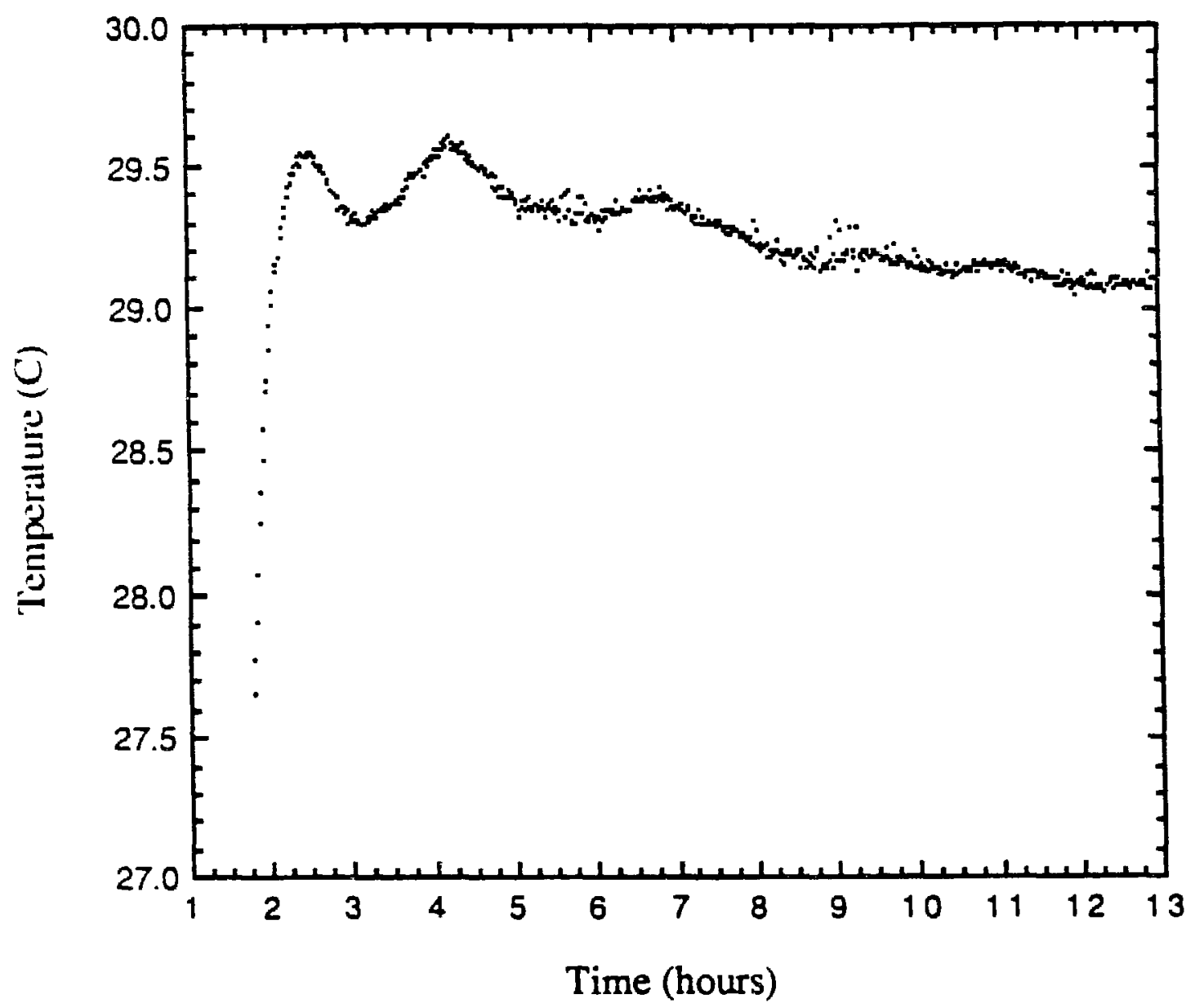

Figure 4.5: Measured temperature of the first RF amplifier as a function of time for the night of Sept 16, 1988. 
The warmer ambient environment prevented the thermal box from fulfilling its original design goal, which was to provide a regulated heat sink for the inner plate. Instead, we disconnected the heaters and regulating circuitry on the thermal box and allowed it to float at a temperature between that of the outside environment and the inner plate, relying on the thermal mass of the box to mediate any abrupt changes in the ambient temperature.

Figure 4.5 shows the temperature of the inner plate as a function of time for a typical night, Sept 16. The temperature rise at the start resulted from the radiometer's electronics being turned on, which caused a slight temperature rise as the heaters drew less power in response to the waste heat dumped by the RF amplifiers. The temperature then remained stable to $0.3 \mathrm{C}$ for the duration of the observations. The small change within the $100-200$ second time scales of individual scans is well fit by a simple linear drift.

The RF chain had a thermal response, with a fractional gain coefficient of $2.8 \% \mathrm{~K}^{-1}$. Typical temperature drifts of $<0.05 \mathrm{~K}$ on 200 second time scales corresponded to a calibration response of $<0.1 \%$. We calibrated the radiometer on comparable time scales, and observed calibration drifts $\delta \mathrm{G} / \mathrm{G} \sim 10^{-4}$. Thermal stability was not a limiting factor.

\subsubsection{Offset Changes}

The entire experiment depended upon the radiometer's response remaining independent of its orientation. We tested extensively to place limits on this effect. In the simplest test, we clamped the ambient calibration target firmly over the antenna aperture, then repeatedly changed the radiometer's position while recording the output. The positions were either those of the zenith sky measurement (up and down) or those of an atmospheric measurement $\left(-40^{\circ},-30^{\circ}\right.$, zenith, $+30^{\circ}$, and $\left.+40^{\circ}\right)$. We varied the amount of time spent on each target from test to test, with the radiometer at each position for either 16 , 32 , or 64 seconds. We used a digital computer to analyze the signal-averaged data for systematic signal changes correlated with position.

The radiometer may be modelled as an ideal radiometer with system temperature

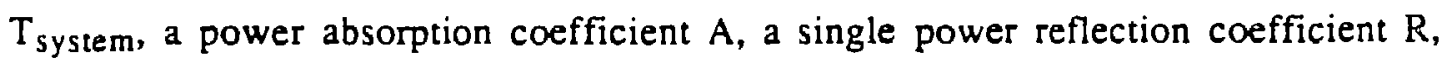
observing a target at temperature $\mathrm{T}_{\text {Target }}$. Neglecting terms second-order in $\mathrm{A}$ and $\mathrm{R}$, the signal output is 


$$
S=\frac{1}{G}\left\{T_{\text {Target }}+T_{\text {system }}+R\left(T_{\text {system }}-T_{\text {Target }}\right)+A\left(T_{\text {int }}-T_{\text {Target }}\right)\right\},
$$

where $T_{\text {int }}$ is the temperature of the internal absorbing component. Coherent reflection effects have been ignored. Changes in the calibration constant $G$, the system temperature, or the reflection or absorption coefficients will produce a signal change, which will be detected if it is systematically correlated with radiometer position.

These tests were sensitive to changes in radiometer calibration constant or system temperature, but were not particularly sensitive to changes in insertion loss or reflection coefficient of the components in the RF chain (the ambient target temperature, the temperature of components in the RF chain, and the system temperature are fairly similar). To test for these effects, the radiometer must view a target with temperature significantly different from the system temperature and the temperature of the RF components. For this purpose we used a small liquid nitrogen (LN) target, which consisted of a disk of microwave absorber (Eccosorb CV-3 backed by two layers of Eccosorb AN-72) in a metal cylinder closed on one end. The target fit neatly over the antenna aperture, with the metal "can" minimizing contributions from the surroundings. Dipped in $\mathrm{LN}$, inverted until the liquid had largely ceased to drip from the Eccosorb tips, and then placed over the antenna aperture, the target proved to be stable in temperature in any orientation for periods greater than 32 seconds.

To prevent $L N$ from dripping into the antenna, we covered the aperture with a polyethylene bag which we replaced as necessary. We then alternately placed the target over the antenna with the radiometer in the up and in the down position, obtaining a minimum of 16 seconds of data at a position. The target remained in the $\mathrm{LN}$ bath between each position. To minimize risks to the radiometer and the operator, we made no attempt at periodicity, but averaged the data from the two positions "by hand" to produce a time sequence which we analyzed for systematic differences between the two positions. We recorded the temperature of the antenna during these tests to determine whether the large drifts in temperature caused by LN drips and splashes caused a significant signal drift.

We performed additional tests to ensure that the $\mathrm{LN}$ target itself did change temperature in a position-dependent fashion. Although its temperature in either orientation was stable in time over the 32-second duration of a single observation, it was possible that LN collecting in the Eccosorb tips could alter the target's antenna temperature. We tested for this effect in two ways. With the radiometer pointing up, we compared the output as 
Ambient Target

\begin{tabular}{|c|c|c|c|c|c|}
\hline Date & $\Delta(180)$ & $\Delta(-40)$ & $\Delta(-30)$ & $\Delta(+30)$ & $\Delta(+40)$ \\
\hline $\begin{array}{l}5 \text { Sept } \\
7 \text { Sept } \\
9 \text { Sept }\end{array}$ & $\begin{array}{c}9 \pm 4 \\
-25 \pm 5 \\
-31 \pm 3 \\
-29 \pm 3\end{array}$ & $\begin{array}{l}-- \\
--- \\
-- \\
--\end{array}$ & $\begin{array}{l}--- \\
-- \\
-- \\
--\end{array}$ & $\begin{array}{l}-- \\
-- \\
--\end{array}$ & $\begin{array}{l}-- \\
-- \\
-- \\
--\end{array}$ \\
\hline $\begin{array}{l}10 \text { Sept } \\
11 \text { Sept } \\
13 \text { Sept } \\
15 \text { Sept }\end{array}$ & $\begin{array}{l}-20 \pm 4 \\
-33 \pm 3 \\
-21 \pm 3 \\
-23 \pm 3\end{array}$ & $\begin{array}{c}23 \pm 5 \\
6 \pm 7 \\
-- \\
--\end{array}$ & $\begin{array}{c}3 \pm 5 \\
2 \pm 8 \\
-- \\
--\end{array}$ & $\begin{array}{c}16 \pm 4 \\
-4 \pm 8 \\
-2 \pm 4 \\
-15 \pm 4\end{array}$ & $\begin{array}{c}17 \pm 4 \\
14 \pm 8 \\
-9 \pm 5 \\
-15 \pm 3\end{array}$ \\
\hline 18 Sept & -- & & & & \\
\hline & --. & --. & --. & $\begin{array}{l}-8 \pm 2 \\
0 \pm 3\end{array}$ & $\begin{array}{l}-8 \pm 6 \\
-2 \pm 5\end{array}$ \\
\hline 19 Sept & -- & --- & -.. & $\begin{array}{c}5 \pm 8 \\
-13 \pm 4\end{array}$ & $\begin{array}{l}12 \pm 6 \\
-9 \pm 4\end{array}$ \\
\hline Mean & $-26 \pm 2$ & $20 \pm 7$ & $2 \pm 2$ & $-1 \pm 3$ & $2 \pm 4$ \\
\hline \multicolumn{6}{|c|}{ LN Target } \\
\hline $\begin{array}{l}8 \text { Sept } \\
13 \text { Sept }\end{array}$ & $\begin{array}{l}-33 \pm 26 \\
-67 \pm 23\end{array}$ & --- & --- & --. & --- \\
\hline Mean & $-52 \pm 17$ & ... & $\ldots$ & $\ldots$ & ... \\
\hline
\end{tabular}

Table 4.4: Results of systematic offset change tests performed at Barcroft, with ambient and $\mathrm{LN}$ targets over the antenna aperture. $\Delta(\theta)$ is the difference $\mathrm{G}\left(\mathrm{S}_{\mathrm{zenith}}-S_{\theta}\right)$, in $\mathrm{mK}$. The means are calculated from the individual measurements without weighting. A positive result means the zenith appears warmer than angle $\theta .180^{\circ}$ is the down position looking into the cold load. 
the radiometer viewed the $\mathrm{LN}$ target with varying amounts of $\mathrm{LN}$ dripping from the Eccosorb. We observed no change at the $15 \mathrm{mK}$ level. In addition, we compared the $\mathrm{LN}$ target in both the up and down positions to an independent cold target (either the reference load with LN as cryogen or the zenith sky). We observed no position-dependent effects at the $30 \mathrm{mK}$ level. We conclude that the $\mathrm{LN}$ target antenna temperature is independent of orientation to better than $30 \mathrm{mK}$.

Table 4.4 shows the results of all offset tests performed at Barcroft. Since all the measurements were differential, we have arbitrarily taken the effect at zenith to be the zero point of the tests. The results were generally consistent from day to day, and had noise levels consistent with control runs in which the radiometer did not move.

The largest effect appeared when the radiometer is inverted. With an ambient target over the antenna, the radiometer output was $26 \pm 2 \mathrm{mK}$ colder when the radiometer pointed down. With a $\mathrm{LN}$ target replacing the ambient target, the effect grew slightly, to $52 \pm 17$ $\mathrm{mK}$ (statistical uncertainty only). Such a behavior was inconsistent with a one-component model of the effect. The simplest cause, a small variation in gain, should scale as the sum of the target and system temperatures and would be expected to decrease by a factor 0.6 when viewing the colder $\mathrm{LN}$ target. Changes in system temperature are independent of target temperature, and should remain constant as the targets are switched. Models with changes in internal reflection coefficients or insertion losses, though, predict a larger signal when the $L N$ target is viewed. A changing reflection coefficient will cause a signal change proportional to the difference between the target and the broadcast temperatures, while a change in insertion loss will cause a signal change proportional to the difference between the target temperature and the temperature of the component causing the insertion loss. Since the broadcast, ambient, and component temperatures are all within $25 \mathrm{~K}$ of each other, both these effects predict a signal increase of an order of magnitude when viewing the LN target. Table 4.5 summarizes these simple one-component models. It is clear that no single model is a good estimator of the actual signal, which increased a factor of $2.0 \pm$ 0.7 viewing the $\mathrm{LN}$ target. Lacking targets at temperatures other than ambient and $\mathrm{LN}$, we were unable to distinguish various combinations of these effects.

Although we did not measure the signal with a $4 \mathrm{~K}$ target, we can obtain upper and lower bounds. An obvious lower bound is a change in calibration constant. Pure reflection or insertion loss changes increase the signal and provide an upper limit. Scaling from the $L N$ results (the closest test of the actual zenith sky measurement) gives 


$$
0.027 \mathrm{~K} \leq \Delta \mathrm{T}_{\text {Offset }} \leq 0.090 \mathrm{~K}
$$

The LN test better approximated a $4 \mathrm{~K}$ load; consequently, we chose to use the results of the LN test, unscaled, to correct the measured signal difference between the cold load and the zenith sky. We took the uncertainty to be the statistical uncertainty of the LN results added in qaudrature with the $0.03 \mathrm{~K}$ limit on $\mathrm{LN}$ target stability. The resultant correction $\Delta T_{\text {Offset }}$ in Eq. 2.7 was then

$$
\Delta \mathrm{T}_{\text {Offset }}=0.052 \pm 0.034 \mathrm{~K}
$$

\subsubsection{Gain Stability}

The tests described above limited the effects of systematic variations correlated with position. For a total-power radiometer, it was also vital that the gain did not change significantly on time scales of a comparison between two targets. The output signal was proportional to the calibration constant and the system temperature,

$$
S \sim \frac{1}{G}\left\{T_{\text {system }}+T_{\text {Target }}\right\}
$$

consequently, a change in calibration constant as small as $10^{-3}$ would cause a signal change of $230 \mathrm{mK}$. To test the stability of the instrument, we recorded the output signal while the radiometer was left undisturbed viewing either an ambient target or the zenith sky (a convenient cold target).

An ideal total-power radiometer with system temperature $T_{\text {system, bandwidth } B \text {, }}$ calibration constant $G$, and integration time $\tau$ viewing a target of antenna temperature T Target will have RMS noise described by

$$
\Delta \mathrm{T}_{\text {noise }}=\left(\mathrm{T}_{\text {system }}+\mathrm{T}_{\text {Target }}\right)\left\{\frac{\mathrm{l}}{\mathrm{B} \tau}+\left(\frac{\delta \mathrm{G}}{\mathrm{G}}\right)^{2}\right\}^{1 / 2}
$$

(Kraus, 1969). If gain fluctuations $\delta G / G$ are negligible, the noise is gaussian and decreases as the inverse square root of the integration time. Short-term changes in calibration will cause an increase in RMS noise that will not necessarily decrease with integration time. By taking data when the radiometer observes a stable target, averaging the data into successively longer blocks, and comparing the resultant RMS scatter to the predicted $\tau^{-1 / 2}$ spectrum, we can assess the effects of short-term changes in calibration. 
The tests showed non-gaussian changes in calibration to be the dominant source of noise in the instrument. For the $234 \mathrm{~K}$ system temperature, $500 \mathrm{MHz}$ bandwidth, and 2 second integration time of the radiometer, the predicted noise was $7 \mathrm{mK}$ between adjacent data points (viewing a $4 \mathrm{~K}$ target). The measured value was $33 \mathrm{nK}$, which decreased approximately as $\tau^{-1 / 2}$ on time scales between 32 and 64 seconds. On longer time scales the noise increased slightly from its minimum value. When the radiometer viewed a stable ambient target, we observed a similar behavior with the magnitude of the noise larger by a factor $2.7 \pm 0.5$.

The effect of such short-term calibration changes on the measurements of $T_{A, z e n i t h}$ and $T_{A, A t m}$ can be assessed directly using data from observations of stable targets. We averaged the data into blocks 24 seconds in duration separated by a time $\Delta t$, and looked for changes in the RMS as the time delay $\Delta t$ was increased. The results are shown in Table 4.6. The 24-second integration time and time delay $\Delta t$ betwen 32 and 112 seconds correspond to the time scales typical of the atmospheric and zenith sky measurements. The noise increased as $\Delta \mathrm{t}^{0.4 \pm 0.1}$, and was larger when viewing the ambient target by a factor $1.8 \pm 0.1$. Both are consistent with a random-walk process in the calibration constant of the radiometer, with variations $\delta \mathrm{G} / \mathrm{G} \sim 10^{-4}$ on time scales of several minutes.

The fluctuations served to inject noise at the $20-30 \mathrm{mK}$ level to the signal differences used to determine $T_{A, z e n i t h}$ and $T_{A, A t m}$. Drifts in the calibration could also systematically alter the mean values by adding a systematic signal

$$
\Delta \mathrm{T}_{\text {gain }}=\frac{\delta \mathrm{G}(\Delta \mathrm{t})}{\mathrm{G}} \mathrm{T}_{\text {system }}
$$

to the difference between two observations spaced a time $\Delta t$ apart, if the gain drifts have a preferred sign. As described below, the data analysis routine included removal of a linear drift in calibration over the course of a single scan. We have analyzed the control runs of stable data as though they were atmospheric or zenith sky measurements, removing the linear component of the drift on time scales of 128 to 224 seconds. The resultant data sets had zero mean and RMS noise consistent with the 20-30 mK noise described above. We concluded that fluctuations in radiometer calibration dominated the system noise, but served only to inject additional noise at the $20-30 \mathrm{mK}$ level and did not systematically alter the signal differences. 


\begin{tabular}{llc}
\hline Variation & Scaling & $\frac{\text { LN Target }}{\text { Ambient Target }}$ \\
\hline Gain & $\frac{\delta \mathrm{G}}{\mathrm{G}}\left\{\mathrm{T}_{\text {system }}+\mathrm{T}_{\text {Target }}\right\}$ & 0.6 \\
\hline System Temperature & $\delta \mathrm{T}_{\text {sys }}$ & 1 \\
\hline Reflection & $\frac{\delta \mathrm{R}}{\mathrm{R}}\left\{\mathrm{T}_{\text {Broadcast }}-\mathrm{T}_{\text {Target }}\right\}$ & 8 \\
\hline Insertion Loss & $\frac{\delta \mathrm{A}}{\mathrm{A}}\left\{\mathrm{T}_{\text {Int }}-\mathrm{T}_{\text {Target }}\right\}$ & $8-30$ \\
\hline
\end{tabular}

Table 4.5: One-component models of systematic position-dependent signal changes. $\mathrm{T}_{\text {sysuem }}$ is the system temperature ( $233 \mathrm{~K}$ ); $\mathrm{T}_{\text {Target }}$ is the temperature of the target viewed (273 K ambient, $73 \mathrm{~K} \mathrm{LN}$ ); $\mathrm{T}_{\text {Broadcast is the broadcast temperature (equal to the temperature }}$ of the isolator, $300 \mathrm{~K}$ ); and $\mathrm{T}_{\mathrm{Int}}$ is the temperature of the radiometer component whose insertion loss changes. Depending on the component, $T_{\text {Int }}$ ranges between ambient (the horn) and $300 \mathrm{~K}$ (RF chain).

\begin{tabular}{ccc}
\hline$\Delta t$ & Zenith Sky & Ambient Target \\
\hline $32 \mathrm{sec}$ & $20 \pm 2 \mathrm{mK}$ & $36 \pm 2 \mathrm{mK}$ \\
$64 \mathrm{sec}$ & $27 \pm 3 \mathrm{mK}$ & $47 \pm 2 \mathrm{mK}$ \\
$112 \mathrm{sec}$ & $32 \pm 5 \mathrm{mK}$ & $57 \pm 6 \mathrm{mK}$ \\
\hline
\end{tabular}

Table 4.6: RMS fluctuations of data sets as a function of time delay $\Delta t$ between them, for both ambient and cold stable targets.

\begin{tabular}{lcccc}
\hline \hline Date & $\begin{array}{c}\mathrm{G}_{\text {LHe-LN }} \\
(\mathrm{mK} / \mathrm{du})\end{array}$ & $\begin{array}{c}\text { GLHe-Ambient } \\
(\mathrm{mK} / \mathrm{du})\end{array}$ & $\begin{array}{c}\mathrm{G}_{\text {LN-Ambient }} \\
(\mathrm{mK} / \mathrm{du})\end{array}$ & $\begin{array}{c}\text { GLHe-LN } \\
\text { GLHe-Ambient }\end{array}$ \\
\hline Sept 8 & $15.76 \pm 0.02$ & $16.08 \pm 0.01$ & $16.19 \pm 0.01$ & 0.980 \\
Sept 13 & $15.12 \pm 0.02$ & $15.28 \pm 0.01$ & $15.35 \pm 0.01$ & 0.990 \\
Sept 14 & $15.03 \pm 0.02$ & $15.19 \pm 0.01$ & $15.25 \pm 0.01$ & 0.986 \\
Sept 14 & $14.99 \pm 0.01$ & $15.18 \pm 0.01$ & $15.24 \pm 0.01$ & 0.987 \\
Sept 17 & $14.80 \pm 0.01$ & $15.09 \pm 0.01$ & $15.18 \pm 0.02$ & 0.981 \\
\hline Mean & $\ldots$ & $\ldots$ & $\ldots$ & $0.985 \pm 0.002$ \\
\hline \hline
\end{tabular}

Table 4.7: Measured calibration and saturation effects. The quoted uncertainties are statistical only. A du is a digitized unit of the recording system, approximately $0.3 \mathrm{mV}$. 


\subsubsection{Gain Linearity}

The major source of non-linear behavior in the RF chain was the detector diode. We have had extensive experience with the HP 8473B diode; typically, non-linearities appear for output signals above 4-5 mV. The radiometer design deliberately kept the RF gain low to keep the diode in the linear regime for all but the ambient target. The signals for LHe and $L N$ loads were in the linear regime of the diode (-2.0 and $-2.9 \mathrm{mV}$ respectively), while the ambient target $(-5.3 \mathrm{mV})$ was slightly saturated.

Scans measuring $T_{A, z e n i t h}$ and $T_{A, A t m}$ calculated the calibration constant $G$ using the ambient-LHe or ambient-sky signal difference, which was slightly saturated. We corrected the calibration of the radiometer by viewing three targets in $\pi$ pid succession: the reference target, $\mathrm{T}_{\mathrm{A}, \text { load }}=3.6 \mathrm{~K}$, the $\mathrm{LN}$ target $\left(\mathrm{T}_{\mathrm{A}, \mathrm{LN}}=73.8 \mathrm{~K}\right)$ and an ambient target $\left(\mathrm{T}_{\mathrm{A}, \mathrm{Amb}}\right.$ $270 \mathrm{~K}$ ). For some tests the zenith sky replaced the cold load as the coldest target. From the three targets, three calibration constants may be determined (Table 4.7). The ratio of calibrations determined by LHe-LN to LHe-ambient targets fixed the correction to be applied to the LHe-ambient scan calibrations for saturation effects at $0.985 \pm 0.002$ (statistical uncertainty only).

Additional systematic uncertainty in the calibration is introduced if the diode response is slightly non-linear even between the two cold targets ( $4 \mathrm{~K}$ and $77 \mathrm{~K}$ ). A reasonable upper limit to gain corrections arising from non-linearities at low input levels may be determined by assuming the non-linear response to be dominated by a quadratic term. Expanding the system response in a Taylor series, the error induced in the saturation correction by neglecting the quadratic term is given by

$$
\frac{\delta G}{G}=\frac{1}{2} \frac{T_{L N}-T_{L H e}}{T_{A m b}-T_{L N}} \frac{G_{L H e-L N}-G_{L N}-A m b}{G_{L H e}-L N}
$$

From Table 4.7, the estimated systematic uncertainty is $\delta \mathrm{G} / \mathrm{G} \leq 0.003$. Added in qaudrature with the systematic uncertainty, the total calibration uncertainty is

$$
\frac{\delta G}{G}=0.004
$$

dominated by uncertainty in the diode saturation curve. Since we measure $T_{A, z e n i t h}$ and $T_{A, A t m}$ with the same instrument, the systematic uncertainty cancels in the subtraction of the atmosphere, leaving only the smaller uncertainty of the sky-cold load difference in the determination of $T_{A, C M B}$. 


\subsubsection{Sidelobe Response}

The ground was the brightest source in the vicinity of the radiometer. Although its contribution was greatly reduced by the corrugated hom antenna, ground radiation entering the antenna sidelobes could still contribute a large, angle-dependent signal to a sky-pointing radiometer, systematically altering the determinations of both $T_{A, z e n i t h}$ and $T_{A, A t m}$. We estimated the contribution of $\mathrm{T}_{\mathrm{A}, \mathrm{Ground}}$ in several ways.

The measured far-field beam pattern convolved with a flat horizon yielded an estimate

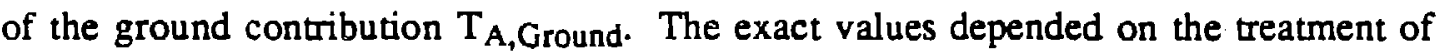
ground emission, and ranged from $0.025 \mathrm{~K}$ for a zenith-pointing radiometer to between 0.03 to $0.05 \mathrm{~K}$ for the radiometer tipped to $40^{\circ}$. A caveat with this method involves the distinction between the near- and far-field response of the radiometer. The ground is within $1.5 \mathrm{~m}$ of the horn aperture, and thus within the near field of the horn. We measured the far-field beam pattem with the pryamidal ground shield in place over the hom. The shield terminates in flared sections with flare radius $8 \mathrm{~cm}$. The ground is far enough from the flared sections that ground emission diffractins over the flares will have substantially the same angular response as the measured far-tield beam paticin. Although the flares are within $1 \mathrm{~m}$ of the hom aperture, they do not move relative to the horn. We conclude that ground emission with the horn/shield combinatior can be modelled to better accuracy than with the hom alone. With the ground treated as an ambient blackbody, the beam response convolved with a flat horizon can be interpreted as ups:r limits to the ground contribution as a function of angle: $T_{A, G r o u n d} \leq 0.03 \mathrm{~K}$ for a zenith-pointing radiometer, and $\leq 0.05 \mathrm{~K}$ at $40^{\circ}$.

This signal was large enough to warrant further testing. At Barcroft, we tested for residual ground radiation diffracting over the ground shield by alternately adding and removing a large piece of sheet metal to one side of the ground shield. The method is analogous to the sidelobe tests described in Chapter 3. We tested both for direct paths to the inner beam ("extension" tests in the nomenclature of $\$ 3.2 .1$ ) and for radiation diffracting around the flared ends of the shields ("trap" tests). The flared sections were not removable; consequently, we were unable to measure the magnitude of radiation blocked by the flared sections, but only the residual diffracting over them.

Sidelobe test results are summarized in Table 4.8. The local horizon rose sharply to the west, and was visible in the main antenna lobe above the ground shield for the $-40^{\circ}$ and $-30^{\circ}$ positions. A small signal was evident in the E-plane at all angles, caused by radiation 


\begin{tabular}{lccccc}
\hline \multicolumn{1}{c}{ Test } & $-40^{\circ}$ & $-30^{\circ}$ & zenith & $+30^{\circ}$ & $+40^{\circ}$ \\
\hline E-plane extension & $84 \pm 12$ & $24 \pm 4$ & $-11 \pm 4$ & --- & --- \\
E-plane trap & $12 \pm 5$ & $-12 \pm 9$ & $3 \pm 3$ & $5 \pm 2$ & $10 \pm 2$ \\
H-plane trap & -- & $-3 \pm 2$ & $-1 \pm 3$ & $-5 \pm 3$ & $-5 \pm 3$ \\
\hline \hline Total : & $96 \pm 15$ & $24_{-4}^{+14}$ & $3_{-3}^{+8}$ & $5_{-2}^{+5}$ & $10_{-2}^{+5}$ \\
\hline Be.um Pattern: & --- & -- & $23 \pm 5$ & $36 \pm 5$ & $47 \pm 10$ \\
\hline \hline
\end{tabular}

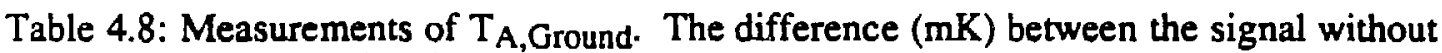
the extra shield and the signal with the extra shield in place is shown for each of the atmospheric scan angles. Ground radiation blocked by the extra shield has a positive sign. The local horizon rises above the ground shield for the western (-) angles, causing a large direct signal. The last row indicates the maximum signal predicted by a convolution of the measured far-field beam pattern with a flat blackbody horizon.

\begin{tabular}{|c|c|c|c|c|c|}
\hline \multicolumn{6}{|c|}{$\mathrm{T}_{\mathrm{A}, \mathrm{Ground}}(\mathrm{mK})$} \\
\hline & $-40^{\circ}$ & $-30^{\circ}$ & zenith & $+30^{\circ}$ & $+40^{\circ}$ \\
\hline Upper Limit & -- & - & $23 \pm 5$ & $36 \pm 5$ & $47 \pm 10$ \\
\hline Lower Limit & $96 \pm 15$ & $24_{4}^{+14}$ & $3+3$ & $5+5$ & $10_{-2}^{+5}$ \\
\hline Mean & $125 \pm 23$ & $45 \pm 18$ & $13 \pm 10$ & $21 \pm 15$ & $29 \pm 18$ \\
\hline \multicolumn{6}{|c|}{$\Delta T_{A, \text { Ground }}(\theta-$ zenith $)(\mathrm{mK})$} \\
\hline Upper Limit & --- & --- & -. & $13 \pm 7$ & $24 \pm 11$ \\
\hline Lower Limit & $93 \pm 16$ & $21+14$ & -- & $2+6$ & $7+5$ \\
\hline Mean & $112 \pm 19$ & $32 \pm 16$ & $\ldots$ & $8 \pm 8$ & $16 \pm 13$ \\
\hline
\end{tabular}

Table 4.9: Estimated range of $T_{A . G r o u n d}$ for the atmospheric scan angles. Upper limits are estimated from the beam pattern convolved with a flat horizon, which is valid for the eastern ( + ) angles. Lower limits are the results of sidelobe tests. The mean values are used to remove $T_{A, G r o u n d}$ from the total sky signal. 
diffracting over the flared sections of the ground shields. We observed only small null signals in the $\mathrm{H}$-plane.

The total measured $T_{A, G r o u n d}$ for each angle was the linear sum of the effect measured in the $\mathrm{E}$ - and $\mathrm{H}$-plane tests, with the H-plane counted twice as it contributed equally on either side of the square-aperture shield. Negative (null) signals were counted as zero. Sidelobe response should be similar on opposite sides of the shields for the H-plane at all angles and for the E-plane at the zenith. We therefore treated the uncertainties from these positions as correlated and added them linearly, effectively doubling the error estimates for these positions. The total estimated uncertainty at each angle was then the quadrature sum of the E-plane and H-plane uncertainties (e.g., the uncertainty at the zenith was the quadrature sum of twice the uncertainty of each row of Table 4.8 , while the uncertainty at $+40^{\circ}$ was the quadrature sum of the E-plane tap and twice the H-plane trap uncertainties). As the ground cannot subtract signals from the zenith, null results added to the positive uncertainty only.

The measured value of $T_{A, G r o u n d}$ was somewhat lower than the value expected from the beam pattern. With the radiometer pointed at the zenith, we observed $3_{-3}^{+14} \mathrm{mK}$ ground

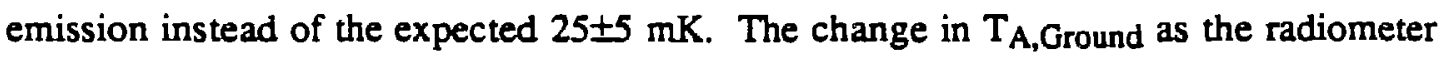
tipped showed similar behavior; at $30^{\circ}$ we observed a signal of $5+5 \mathrm{mK}$ instead of $36 \pm 5 \mathrm{mK}$, and at $40^{\circ}$ we observed $10_{-2}^{+5} \mathrm{mK}$ instead of $47 \pm 10 \mathrm{mK}$.

The two results can be reconciled if the direct sidelobe tests did not modulate the entire diffracted ground signal. Given the null results in the H-plane, we cannot rule out this possibility. The direct tests, then, became a lower limit to $T_{A, G r o u n d}$ while the beam pattern convolution formed an upper limit. We took as the estimate of $T_{A \text {,Ground the }}$ arithmetic mean of the two methods, with an uncertainty equal to half the spread between them. A substantial fraction of $T_{A, G r o u n d}$ was a constant arising from the response at large angles $\left(2130^{\circ}\right)$ which always viewed the ground for any of the radiometer positions used. This contribution did not change as the radiometer is tipped; hence, the differential

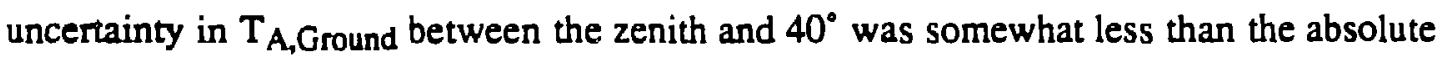
uncertainty at either position. We estimated the differential uncertainty as the quadrature sum of the uncertainties in the differential signal calculated from the upper and lower limits. The estimated values for $T_{A, G r o u n d}$, both absolute and differential, are summarized in Table 4.9. 


\subsubsection{RFI}

Another unwanted contribution to the sky signal was RFI. Near major population centers, commercial and government microwave communication links raised $\mathrm{T}_{\mathrm{A}, \text { zenith }}$ to over $50 \mathrm{~K}$. The situation was considerably better at a remote location; nevertheless, we searched for RFI at Barcroft before beginning any other tests. With a spectrum analyzer replacing the detector diode, we observed no RFI at $10 \mathrm{kHz}$ resolution over the range $5.75-8.75 \mathrm{GHz}$, coiresponding to a limit on RFI contributions of $\mathrm{T}_{\mathrm{A}, \mathrm{RFI}}<0.005 \mathrm{~K}$.

\subsubsection{Pointing}

Systematic differences between the zenith angle $\theta$ used in atmospheric analysis and the true zenith angle $\theta^{\prime}$ of the radiometer could alter the calculated $T_{\text {A,Atm }}$ from the true value. We measured the pointing of the radiometer repeatedly and found it to be quite stable over both long and short time scales (Table 4.3). The large ground shield precluded us from measuring the pointing with a precision bubble clinometer when the ground shield was over the antenna, opening the possibility of a systematic change in pointing when the ground shield was in place. We tested for this effect by using the output of a precision electronic clinometer mounted inside the radiometer.

We calibrated the electronic clinometer (Accustar \#2383-01) by recording its output simultaneously with the measurement of the pointing using the bubble clinometer. The Accustar output was linear to $0.4 \%$ at angles as large as $40^{\circ}$. We then recorded the Accustar output with the ground shield on and off; the mean difference in pointing was 2 ', within the limits of the measurement. We concluded that the addition of the ground shield did not significantly change the pointing of the radiometer. The bubble clinometer used to establish the absolute pointing has an uncertainty of \pm 5 ' in its zero point. This uncertainty dominates the pointing uncertainty.

\subsubsection{Cross-talk}

The radiometer shared the recording system with radiometers operating at $1.5,3.8$, and $90 \mathrm{GHz}$, as well as temperature information from the reference cold load. We tested for cross-talk between the instruments. While the $7.5 \mathrm{GHz}$ radiometer observed a stable ambient target, we alternately covered and uncovered the antenna of one of the other radiometers with an ambient target, causing a large square-wave output signal for that 
radiometer. We then examined the output of the $7.5 \mathrm{GHz}$ radiometer for any signals synchronous with this square-wave signal. We observed no effects at the $5 \mathrm{mK}$ level.

\subsection{Data Reduction and Analysis}

\subsubsection{Zenith Sky}

We calculated $\mathrm{T}_{\mathrm{A}, z e n i t h}$ using Eq. 2.5 on a scan-by-scan basis. The recorded signal from the internal electronic clinometer served to differentiate the up and down positions. We discarded data taken between positions, and any data krown to be contaminated (e.g., a missed sequence noted in the log). We calibrated the radiometer once per scan using the cold load and the ambient target (Eq. 2.4). Subsequent analysis used a calibration constant $\mathrm{G}(\mathrm{t})$ interpolated between successive calibrations. The analysis algorithm followed the sequence:

- Calculate the calibration constant $G$ for the current scan and the next scan using data from the reference load and ambient target, including the correction for saturation. Interpolate to determine $G(t)$ throughout the current scan.

- Determine $T_{A, z e n i t h}$ (Eq. 2.5) using $G(t)$ and the signals from the zenith sky and the reference load.

- Determine the system temperature using $G(t)$ and the signal from the reference load.

\subsubsection{Atmosphere}

We determined $\mathrm{T}_{\mathrm{A}, \mathrm{Atm}}$ from Eq. 2.9 on a similar scan-by-scan basis using procedures similar to those previously described. The analysis followed the algorithm:

- Use the zenith sky and ambient target to calculate the calibration constant $G$ for the current scan and the next scan, assuming T $_{A, z e n i t h}$ to be the sum of a $2.75 \mathrm{~K}$ blackbody and the last determination of $\mathrm{T}_{\mathrm{A}, \mathrm{Atm}}$. Include the correction for saturation. Interpolate to determine $G(t)$ for the current scan. 
- Determine $T_{A, A t m}$ for each of the scan angles used. Define the mean

$\mathrm{T}_{\mathrm{A}, \mathrm{Atm}}$ as the arithmetic mean of the $+30^{\circ}$ and $+40^{\circ}$ (east) positions.

- Determine the system temperature using $G(t)$ and the signal from the ambient target.

- Correct the raw data for systematic offset changes, sidelobe contribution, galactic contribution, and differential pointing.

- Re-determine $G(t)$ using the corrected data and the previous estimate of $T_{A, A t m}$

- Re-determine $T_{A, A t m}$ and $T_{\text {system }}$ using the corrected data and the revised $G(t)$.

We did not use the western (-) angles in the mean $T_{A, A t m}$ since those angles had a much larger correction for sidelobe contributions. The determination of $G(t)$ was less precise for atmospheric scans as the temperature of the cold target (the zenith sky) was not known to the same precision as the reference targec. $R M S$ variations in $T_{A, A t m}$ of magnitude $0.2 \mathrm{~K}$ caused a fractional calibration uncertainty of $\delta \mathrm{G} / \mathrm{G} \sim 10^{-3}$. The concomitant uncertainty in $\mathrm{T}_{\mathrm{A}, \mathrm{Atm}}$ is small ( $\left.1 \mathrm{mK}\right)$.

The linear interpolation for $G(t)$ removed the major effects of gain drifts during the course of a single scan. Non-linear changes large enough to effect the estimate of $T_{A, A t m}$ occurred rarely. The pattern of the sky observations (western angles first followed by the zenith and the eastem angles) provided a distinctive signature for such processes. The output signal when observing a cold target was dominated by the system temperature:

$$
S \sim \frac{1}{G(t)}\left[T_{\text {system }}+T_{\theta}\right] \sim \frac{1}{G(t)} T_{\text {system }}
$$

Residual drifts in $G(t)$ on time scales of a single scan typically will add a signal $\Delta S$ to the west-zenith signal difference, and a signal $-\Delta S$ of the opposite sign to the east-zenith signal difference, which occurred in the opposite time order. The calculated values for $T_{A, A t m}$ should be displaced by roughly equal amounts above and below the mean value. We observed this signature in 10 of the 285 total scans. The suspect scans were discarded.

\subsubsection{Galaxy}

We obtained a differential profile of the galaxy by combining data from the nights of 1988 Sept 5, 6, 8, and 12 using Equation 2.16. The analysis followed the algorithm 
- Use the position sense switches on the turntable to determine the pointing (east, west, or in transit). Select the east position.

- Examine the next two data records and reject any scans that fail to follow the expected pattern (east, move, west, move).

- Determine the calibration constant by interpolating between the most rec 'nt calibrations. Use the calibration and the east/west signal difference to determine the difference in galactic antenna temperature.

- Use the recorded time to bin the temperature difference by the right ascension of the east beam. We used a bin width of $4^{\circ}$, allowing each binned sky position to be observed for 15 independent scans per night. Over the course of the four nights, each bin accumulated between 20 and 50 independent observations of the galactic temperature difference.

The differential local horizon can conceivably add a constant signal difference to the sky difference. We did not obtain the complete sky coverage needed to enforce closure on the data. Instead, we calculated the mean of all difference pairs, and arbitrarily removed the resultant value of $12.4 \mathrm{mK}$ from the data set.

\subsection{Results}

\subsection{1 $\mathrm{T}_{\text {A,zenith }}$}

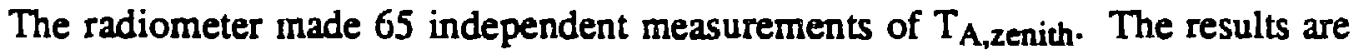
presented in Table 4.10, without corrections for systematic effects. The data from various nights were in good agreement, showing no more variation than would be expected from daily atmospheric changes.

\subsection{2 $\mathrm{T}_{\text {A.Galaxy }}$}

The measured differential galactic signal between R.A. of $-60^{\circ}$ and $+112^{\circ}$ is shown in Figure 4.6, along with the results of a simple galactic model. The double-lobed choppedbeam signature towards the galactic plane is clearly visible. The galactic model is an extrapolation of $408 \mathrm{MHz}$ maps of Haslam et al.(1982), scaled by a position-independent spectral index of -2.75 , and a compilation of thermal sources at $2.7 \mathrm{GHz}$, with spectral index -2.1. We performed a least-squares fit between the model and the data using as free 


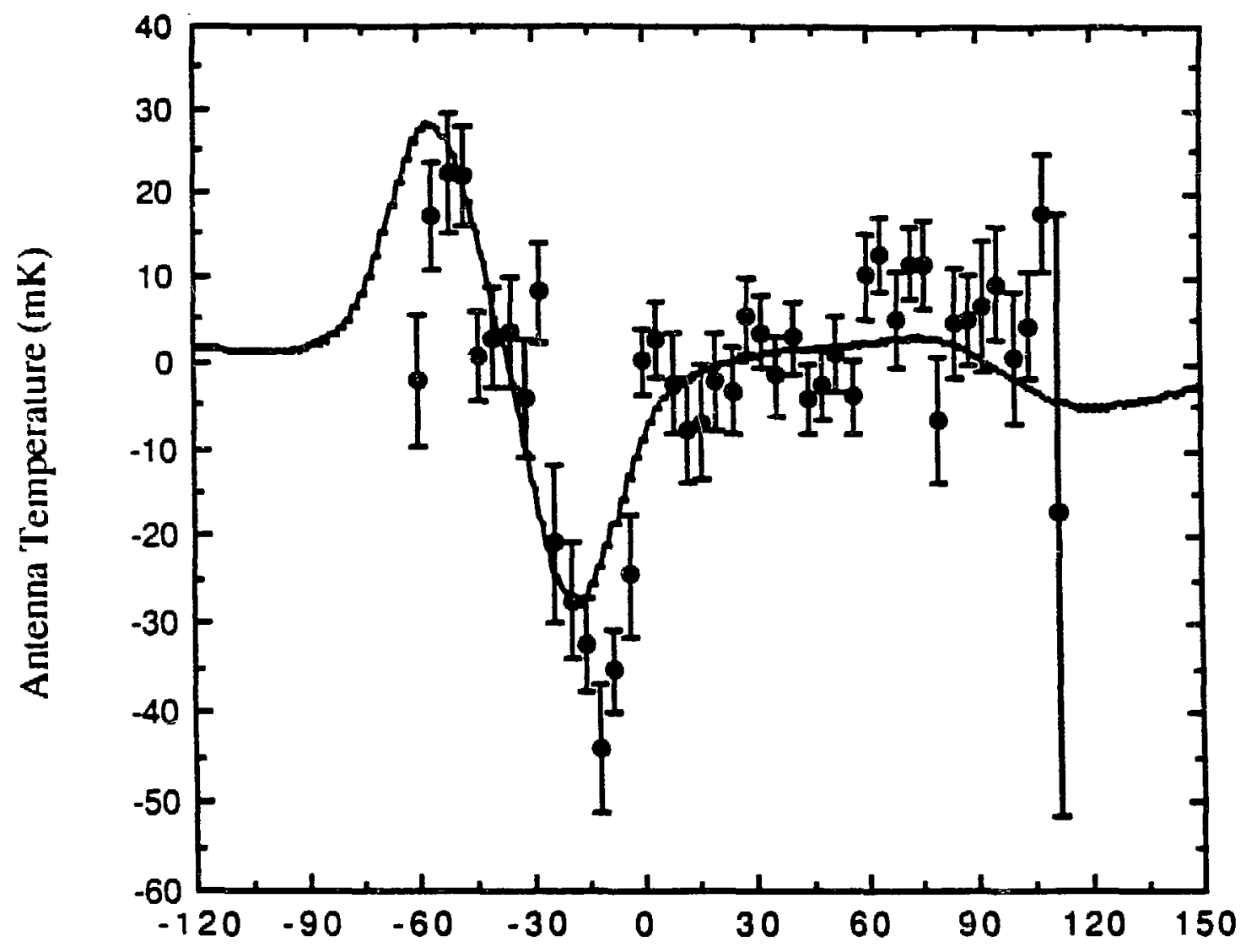

Right Ascension (degrees)

Figure 4.6: Observed differential galactic profile (points) and the signal predicted from extrapolation of maps at lower frequencies (solid line). 


\begin{tabular}{lcccccc}
\hline Date & $\begin{array}{c}\text { Time } \\
\text { (UT) }\end{array}$ & $\begin{array}{c}\text { Number } \\
\text { of Scans }\end{array}$ & $\begin{array}{c}\text { Calibration } \\
(\mathrm{mK} / \mathrm{du})\end{array}$ & $\begin{array}{c}\mathrm{G}\left(\mathrm{S}_{\text {zenith }}-\mathrm{S}_{\text {load }}\right) \\
(\mathrm{mK})\end{array}$ & $\begin{array}{c}\mathrm{T}_{\text {Alload }} \\
(\mathrm{mK})\end{array}$ & $\begin{array}{c}\mathrm{T}_{\text {A,zenith }} \\
(\mathrm{mK})\end{array}$ \\
\hline Sept 16 & $5: 45-6: 15$ & 16 & $14.947 \pm 0.001$ & $-113 \pm 10$ & 3613 & $3506 \pm 10$ \\
Sept 16 & $8: 28-9: 13$ & 21 & $14.827 \pm 0.002$ & $-153 \pm 11$ & 3613 & $3466 \pm 11$ \\
Sept 17 & $11: 45-12: 07$ & 11 & $14.699 \pm 0.004$ & $-156 \pm 13$ & 3613 & $3490 \pm 12$ \\
Sept 19 & $10: 13-10: 47$ & 17 & $14.720 \pm 0.004$ & $-169 \pm 12$ & 3613 & $3460 \pm 12$ \\
\hline
\end{tabular}

Table 4.10: Signal difference and zenith antenna temperature measured at Barcroft in 1989. The quoted uncertainties are statistical only and assume a gaussian distribution. No

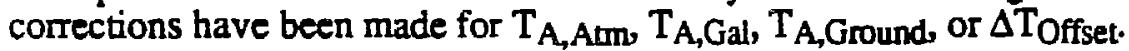

\begin{tabular}{lcccccc}
\hline Date & $\begin{array}{c}\text { Time } \\
(\mathrm{UT})\end{array}$ & $\begin{array}{c}-40^{\circ} \\
(\mathrm{mK})\end{array}$ & $\begin{array}{c}-30^{\circ} \\
(\mathrm{mK})\end{array}$ & $\begin{array}{c}+30^{\circ} \\
(\mathrm{mK})\end{array}$ & $\begin{array}{c}+40^{\circ} \\
(\mathrm{mK})\end{array}$ & $\begin{array}{c}\text { Mean TAAtm } \\
(\mathrm{mK})\end{array}$ \\
\hline Sept 3 & $8: 55-9: 24$ & $1351 \pm 54$ & $1202 \pm 81$ & $1220 \pm 67$ & $1304 \pm 46$ & $1263 \pm 55$ \\
Sept 4 & $4: 44-5: 21$ & $1451 \pm 55$ & $1330 \pm 67$ & $1108 \pm 88$ & $1118 \pm 47$ & $1114 \pm 66$ \\
& $7: 29-8: 06$ & $1295 \pm 49$ & $1105 \pm 76$ & $1211 \pm 45$ & $1274 \pm 36$ & $1243 \pm 33$ \\
Sept 5 & $4: 57-5: 42$ & $1367 \pm 36$ & $1105 \pm 29$ & $1054 \pm 74$ & $1202 \pm 48$ & $1129 \pm 59$ \\
& $8: 02-8: 21$ & $1233 \pm 48$ & $1032 \pm 75$ & $1194 \pm 56$ & $1135 \pm 60$ & $1165 \pm 50$ \\
Sept 7 & $9: 32-9: 50$ & $1257 \pm 26$ & $1079 \pm 49$ & $1275 \pm 41$ & $1210 \pm 28$ & $1243 \pm 29$ \\
Sept 9 & $8: 34-9: 12$ & $1353 \pm 60$ & $1254 \pm 74$ & $1229 \pm 54$ & $1292 \pm 44$ & $1261 \pm 45$ \\
Sept 11 & $8: 34-9: 14$ & $1148 \pm 74$ & $1024 \pm 78$ & $1065 \pm 95$ & $1158 \pm 82$ & $1112 \pm 87$ \\
Sept 14 & $3: 19-4: 26$ & $1168 \pm 36$ & $953 \pm 57$ & $1227 \pm 58$ & $125 \pm \pm 52$ & $1241 \pm 50$ \\
& $5: 16-5: 46$ & $1192 \pm 43$ & $1043 \pm 100$ & $1108 \pm 99$ & $1183 \pm 59$ & $1146 \pm 74$ \\
& $10: 07-10: 30$ & $1044 \pm 83$ & $795 \pm 100$ & $1237 \pm 106$ & $1285 \pm 86$ & $1261 \pm 93$ \\
Sept 15 & $3: 49-4: 26$ & $1105 \pm 48$ & $893 \pm 66$ & $1276 \pm 77$ & $1288 \pm 38$ & $1282 \pm 55$ \\
& $4: 48-5: 03$ & $1233 \pm 36$ & $1192 \pm 123$ & $1329 \pm 111$ & $1246 \pm 52$ & $1288 \pm 66$ \\
Sept 16 & $3: 15-4: 18$ & $1301 \pm 44$ & $1134 \pm 57$ & $1078 \pm 48$ & $1113 \pm 55$ & $1100 \pm 49$ \\
& $5: 03-5: 28$ & $1113 \pm 37$ & $900 \pm 62$ & $1166 \pm 50$ & $1158 \pm 32$ & $1162 \pm 39$ \\
& $6: 29-7: 05$ & $1123 \pm 53$ & $879 \pm 75$ & $1055 \pm 79$ & $1133 \pm 49$ & $1094 \pm 62$ \\
& $7: 42-8: 15$ & $1215 \pm 58$ & $983 \pm 105$ & $1201 \pm 49$ & $1177 \pm 34$ & $1189 \pm 31$ \\
& $9: 24-10: 01$ & $1139 \pm 53$ & $1008 \pm 44$ & $1160 \pm 67$ & $1113 \pm 43$ & $1137 \pm 49$ \\
& $11: 46-12: 12$ & $1213 \pm 38$ & $894 \pm 48$ & $1035 \pm 58$ & $1091 \pm 37$ & $1064 \pm 45$ \\
Sept 17 & $8: 26-9: 03$ & $1127 \pm 43$ & $838 \pm 64$ & $1245 \pm 63$ & $1243 \pm 40$ & $1244 \pm 48$ \\
& $10: 18-11: 02$ & $1098 \pm 41$ & $869 \pm 84$ & $1081 \pm 58$ & $1160 \pm 26$ & $1121 \pm 39$ \\
& $12: 38-13: 08$ & $1129 \pm 56$ & $808 \pm 80$ & $913 \pm 86$ & $990 \pm 35$ & $952 \pm 57$ \\
Sept 19 & $9: 07-10: 03$ & -- & -- & $1046 \pm 42$ & $1095 \pm 24$ & $1071 \pm 31$ \\
& $11: 02-11: 48$ & --- & -- & $1088 \pm 43$ & $1080 \pm 34$ & $1084 \pm 33$ \\
\hline & & & & & &
\end{tabular}

Table 4.11: Measured atmospheric antenna temperatures at each of the $4 \mathrm{scan}$ angles. The mean value for $T_{A, A t m}$ is the arithmetic mean of the easterm angles $\left(+30^{\circ}\right.$ and $\left.+40^{\circ}\right)$. The data have been corrected for $T_{A, G r o u n d} T_{A, G a l a x y}$, and $\Delta T_{\text {Offset. }}$ Quoted uncertainties are statistical only and assume a gaussian distribution. Systematic uncertainties are indicated in Table 4.12. 
parameters the timing offset $\alpha_{0}$, signal offset $\Delta T_{0}$, and position-invariant multiplicative constants for the thermal and synchrotron components:

$$
\operatorname{Data}(\alpha)=\Delta T+A^{*} \operatorname{Synch}\left(\alpha-\alpha_{0}\right)+B^{*} H \Pi\left(\alpha-\alpha_{0}\right)
$$

where $\operatorname{HII}(\alpha)$ and $\operatorname{Synch}(\alpha)$ are the differential galactic profiles at right ascension $\alpha$ predicted by our simple model. The multiplicative constants $A$ and $B$ can be interpreted as position-inpendent corrections to the spectral indices assumed in the simple model. The resultant values which minimized the $\chi^{2}$ of the data were

$$
\begin{aligned}
& \Delta \mathrm{T}_{0}=-0.5 \pm 0.2 \mathrm{mK} \\
& \alpha_{0}=4 \pm 2 \text { minutes } \\
& \mathrm{A}=0.65 \pm 0.06 \\
& B=1.06 \pm 0.06
\end{aligned}
$$

The fit to the data was not particularly impressive $\left(\chi^{2}=108\right.$ for 40 DOF compared to $\chi^{2}=130$ for 44 DOF for the simple model). The signal was above the noise level only in the galactic plane, which was dominated by thermal emission; in this region, we observed $0.89 \pm 0.06$ of the expected ( $\mathrm{HI}+$ synchrotron) signal. Lacking better sensitivity in regions off the galactic plane where synchrotron emission is dominant, we were unable to distinguish between the thermal and synchrotron components within the plane.

The differential galactic scans confirmed that our simple model was not in serious error, as the measured galactic signal lay within $10 \mathrm{mK}$ of the predicted signal (Figure 4.6). We used this simple model to subtract the differential galactic signal from the atmospheric and zenith sky scans. The maximum error entailed is $0.05 \mathrm{~K}$ in $\mathrm{T}_{\mathrm{A}, \mathrm{Atm}}$ and $0.01 \mathrm{~K}$ in $T_{A, z e n i c h}$ if the galactic plane crossed the zenith during observations. Uncertainties in the galactic signal off the plane of the galaxy (i.e., during most of our atmosphere scans and all of the CMB scans) were much smaller (0.005 K).

\subsection{3 $\mathrm{T}_{\mathrm{A}, \mathrm{Atm}}$}

The radiometer performed numerous atmospheric scans at Barcroft. The values for $\mathrm{T}_{\mathrm{A}, \mathrm{Aum}}$ at each of the fours scan angles are presented in Table 4.11 for all nights in which we measured the atmosphere. The angles to the west had a large correction for $T_{A, G r o u n d}$ the mean value for TA,Atm included data from the eastern (+) angles exclusively. The eastern angles were in excellent agreement with each other. $T_{A, A t m}$ measured at $+40^{\circ}$ was 
$20 \pm 12 \mathrm{mK}$ warmer than the value measured at $30^{\circ}$. There was no statistical evidence for unidentified systematic offsets in the mean atmospheric data.

The data at $30^{\circ}$ had a mean RMS scatter of $221 \mathrm{mK}$, while the RMS at $40^{\circ}$ was $162 \mathrm{mK}$. This noise was consistent with the small fluctuations in the calibration constant discussed in $\$ 4.4 .3$, multiplied by the $[\operatorname{secant}(\theta)-1]^{-1}$ angular dependence of the amospheric signal differences, and approximated a gaussian distribution.

The error budget for each atmospheric angle is given in Table 4.12. Systematic corrections are presented as an additive correction to $T_{A, A t m}$. The dominant terms are the differential contributions of $T_{A, G r o u n d}$ and $\Delta T_{\text {Offset }}$ between the zenith and scan angle $\theta$. All the terms in Table 4.12 have been measured; the associated uncertainties represent the $68 \%$ confidence level of the measurement.

\subsection{4 $\mathrm{T}_{\mathrm{A}, \mathrm{CMB}}$}

We determined $T_{A, C M B}$ by solving Eq. 2.7 on a scan-by-scan basis. Each of the terms in Eq. 2.7 has been measured, some in more than one fashion. The estimated error budget for the CMB measurement is given in Table 4.13. The systematic uncertainty of $0.069 \mathrm{~K}$ was dominated by the uncertainty in the largest foreground signal, the atmosphere. We did not measure $T_{A, A m}$ concurrently with $T_{A, \text { zenith}}$; instead, we used the values for $T_{A, A t m}$ from each night fitted to a linear drift. We did not observe the atmosphere to vary dramatically throughout any night; the uncertainty in $\mathrm{T}_{\mathrm{A}, \mathrm{CMB}}$ incurred in the atmospheric interpolation was small. The resultant values for $T_{A, C M B}$ are given in Table 4.14, both for each of the four observing runs, and as a complete data set.

The individual values for $T_{A, C M B}$ had a gaussian distribution (Figure 4.7). The RMS width of the distribution was $0.050 \mathrm{~K}$ with 65 data points; consequently, we adopted $0.006 \mathrm{~K}$ as the estimate of the statistical uncertainty in the mean value of $T_{A, C M B}$. Adding the statistical uncertainty in quadrature with the $0.072 \mathrm{~K}$ systematic uncertainty, we obtained a value for the antenna temperature of the $\mathrm{CMB}$ of

$$
\mathrm{T}_{\mathrm{A}, \mathrm{CMB}}=2.413 \pm 0.072 \mathrm{~K}(68 \% \text { C.L. })
$$

Using Eq. 2.2 to convert this value to thermodynamic temperature yields the final result of

$$
T_{\text {CMB }}=2.59 \pm 0.07 \mathrm{~K}(7.5 \mathrm{GHz})
$$




\begin{tabular}{|c|c|c|c|c|c|}
\hline \multirow[t]{2}{*}{ Effect } & \multirow[t]{2}{*}{ Uncertainty } & \multicolumn{3}{|c|}{ Correction to $\mathrm{T}_{\mathrm{A}, \mathrm{Atm}}(\mathrm{mK})$} & \multirow[b]{2}{*}{$+40^{\circ}$} \\
\hline & & $-40^{\circ}$ & $-30^{\circ}$ & $+30^{\circ}$ & \\
\hline$T_{\text {A,Ground }}$ & $13 \mathrm{mK}$ & $-359 \pm 61$ & $-201 \pm 101$ & $-52 \pm 52$ & $-52 \pm 42$ \\
\hline$\Delta \mathrm{T}_{\text {Offset }}$ & $5 \mathrm{mK}$ & $+64 \pm 22$ & $+12 \pm 12$ & $-6 \pm 20$ & $+6 \pm 13$ \\
\hline$T_{A, G a l a x y}$ & $3 \mathrm{mK}$ & $0 \pm 10$ & $0 \pm 18$ & $0 \pm 18$ & $0 \pm 10$ \\
\hline Beam Pattem & $20 \pm 2^{\circ}$ FWHM & $0 \pm 14$ & $0 \pm 14$ & $0 \pm 14$ & $0 \pm 14$ \\
\hline Pointing & $\pm 5^{\prime}$ & $0 \pm 7$ & $0 \pm 7$ & $0 \pm 7$ & $0 \pm 7$ \\
\hline Absolute Gain & $\pm 0.4 \%$ & $0 \pm 4$ & $0 \pm 4$ & $0 \pm 4$ & $0 \pm 4$ \\
\hline \multicolumn{2}{|c|}{ Total Systematics } & $-295 \pm 68$ & $-189 \pm 105$ & $.58 \pm 61$ & $-46 \pm 48$ \\
\hline \multicolumn{2}{|c|}{ Statistical Uncertainty } & 47 & 69 & 59 & 37 \\
\hline \multicolumn{2}{|c|}{ Total Uncertainty } & 83 & 126 & 85 & 61 \\
\hline
\end{tabular}

Table 4.12: Contributions to the atmospheric error budget. Systematic corrections have been multiplied by the [ $\sec (\theta)-1]^{-1}$ factor; the uncertainties have been added in quadrature. The statistical uncertainty for each angle is the mean value from all scans Sept $16-19$.

\begin{tabular}{llc}
\hline Effect & Magnitude & Uncertainty (mK) \\
\hline$T_{\text {A,Atm }}$ & $1083 \mathrm{mK}$ & 55 \\
$\Delta$ TOffset $_{\text {A,Ground }}$ & $52 \mathrm{mK}$ & 34 \\
$\mathrm{~T}_{\mathrm{A}, \mathrm{Ladd}}$ & $3 \mathrm{mK}$ & 20 \\
$\mathrm{~T}_{\mathrm{A}, \mathrm{Galaxy}}$ & $3629 \mathrm{mK}$ & 9 \\
$\mathrm{~T}_{\mathrm{A}, \mathrm{RFI}}$ & $10 \mathrm{mK}$ & 5 \\
Calibration & $0 \mathrm{mK}$ & 5 \\
& $15 \mathrm{mK} / \mathrm{du}$ & $<1$ \\
Total Systematics & & 69 \\
\hline Statistical Uncertainty & $\ldots$ & 6 \\
\hline Total Uncertainty & & 69 \\
\hline \hline
\end{tabular}

Table 4.13: Contributions to the $\mathrm{CMB}$ error budget. All uncertainties are $68 \%$ confidence level estimates. 


\subsection{Discussion}

This experiment has provided useful measurements of the CMB temperature and the two largest foreground signals, the atmosphere and the galaxy, each of which can be compared to nearby measurements and to theory.

\subsubsection{The Galaxy}

At $7.5 \mathrm{GHz}$, the differential signal was dominated by thermal emission in the plane of the galaxy. The differential galactic measurements agreed with a simple extrapolation from lower frequencies to within $0.01 \mathrm{~K}$. At lower frequencies, synchrotron emission becomes observable off the galactic plane. Simultaneous with the $7.5 \mathrm{GHz}$ scans, similar instruments at 1.5 and $3.8 \mathrm{GHz}$ performed identical differential galactic scans. The results of the low-frequency scans are not yet available; however, the combined galactic profiles will provide a stricter test of galactic emission than the relatively noisy $7.5 \mathrm{GHz}$ scans alone.

\subsubsection{The Atmosphere}

The atmosphere at low frequencies is not completely understood. The dominant component of atmospheric emission below $10 \mathrm{GHz}$ is continuum $\mathrm{O}_{2}$ emission, with a minor variable contribution from water vapor. Models of atmospheric emission with water vapor content typical of our high-altitude site $\left(2-5 \mathrm{~mm} \mathrm{H}_{2} \mathrm{O}\right.$ ) predict $\mathrm{T}_{\mathrm{A}, \mathrm{A} u \mathrm{~m}}$ at $7.5 \mathrm{GHz}$ in the range $0.89-0.96 \mathrm{~K}$ (Liebe $1988,1985,1981$ ). The mean $\mathrm{T}_{\mathrm{A}, \mathrm{Alm}}$ at $7.5 \mathrm{GHz}$ measured during LHe observations was $1.08 \pm 0.02 \mathrm{~K}$. From day to day, we observed RMS scatter of $\sim 0.07 \mathrm{~K}$. Both the magnitude and variability of this signal were slightly larger than predicted.

An increase in signal magnitude and variability compared to modelled atmospheric emission is a feature common to several recent lov-frequency measurements of the atmosphere (e.g. Kogut et al. 1988, De Amici et al. 1988). In general, the model parameters are fitted to the line features of the emission peaks, and may be much worse describing the windows of astrophysical interest. Several rather ad hoc corrections to the contribution from the $\mathrm{O}_{2}$ continuum and the $22 \mathrm{GHz}$ water line have been proposed (c.g., Danese and Partridge 1989 and references therein), which tend to increase the signal 


\begin{tabular}{lcccc}
\hline Date & $\begin{array}{c}\text { Time } \\
(\mathrm{UT})\end{array}$ & $\begin{array}{c}\text { Number } \\
\text { of Scans }\end{array}$ & $\begin{array}{c}\mathrm{T}_{\mathrm{A}, \mathrm{Atm}} \\
(\mathrm{K})\end{array}$ & $\begin{array}{c}\mathrm{T}_{\mathrm{A}, \mathrm{CMB}} \\
(\mathrm{K})\end{array}$ \\
\hline Sept 16 & $5: 45-6: 15$ & 16 & $1.097 \pm 0.025$ & $2.426 \pm 0.010$ \\
Sept 16 & $8: 28-9: 13$ & 21 & $1.085 \pm 0.025$ & $2.404 \pm 0.011$ \\
Sept 17 & $11: 45-12: 07$ & 11 & $1.071 \pm 0.025$ & $2.442 \pm 0.014$ \\
Sept 19 & $10: 13-10: 47$ & 17 & $1.078 \pm 0.025$ & $2.394 \pm 0.012$ \\
\hline All Scans & & $\mathbf{6 5}$ & $\mathbf{1 . 0 8 3 \pm 0 . 0 1 2}$ & $\mathbf{2 . 4 1 3} \pm \mathbf{0 . 0 0 6}$ \\
\hline \hline
\end{tabular}

Table 4.14: Antenna temperature of the atmosphere and CMB. The quoted uncertainties are statistical only to allow comparison of data between days.

\begin{tabular}{llcc}
\hline Year & $3.8 \mathrm{GHz}$ & $7.5 \mathrm{GHz}$ & $10 \mathrm{GHz}$ \\
\hline 1986 & $870 \pm 108$ & -- & $1200 \pm 65$ \\
1987 & $950 \pm 101$ & -- & $1160 \pm 83$ \\
1988 & $970 \pm 100$ & $1080 \pm 55$ & - \\
\hline Model & $810-830$ & $890-960$ & $990-1120$ \\
\hline
\end{tabular}

Table 4.15: Comparison of $T_{A, A t m}(\mathrm{mK})$ measured from Barcroft with model predictions. The quoted uncertainties are $68 \%$ confidence level and include systematic effects. Model predictions assume a range of precipitable water vapor between $2-5 \mathrm{~mm}$.

\begin{tabular}{lccc}
\hline Reterence & $\begin{array}{c}\text { Wavelength } \\
\text { (cm) }\end{array}$ & $\begin{array}{c}\text { Frequency } \\
\text { (GHz) }\end{array}$ & $\begin{array}{c}\text { TCMB } \\
\text { (K) }\end{array}$ \\
\hline Sironi er al. 1987 & 50.0 & 0.6 & $2.98 \pm 0.55$ \\
Bensadoun (Smoot et al. 1989) & 20.3 & 1.47 & $2.50 \pm 0.19$ \\
Sironi and Bonelli 1986 & 12.0 & 2.5 & $2.79 \pm 0.15$ \\
De Amici (Smoot et al. 1989) & 7.9 & 3.8 & $2.63 \pm 0.11$ \\
Mandolesi et al. 1986 & 6.3 & 4.75 & $2.70 \pm 0.07$ \\
Kogut 1989 & 4.0 & 7.5 & $2.59 \pm 0.07$ \\
Kogut 1989 & 3.0 & 10.0 & $2.62 \pm 0.06$ \\
Johnson and Wilkinson 1986 & 1.2 & 24.8 & $2.783 \pm 0.025$ \\
De Amici et al. 1985 & 0.909 & 33.0 & $2.81 \pm 0.12$ \\
\hline \hline
\end{tabular}

Table 4.16: Results of recent low-frequency CMB measurements. 
magnitude and variability below $10 \mathrm{GHz}$. The resultant variability in $\mathrm{T}_{\mathrm{A}, \mathrm{Atm}}$ at $7.5 \mathrm{GHz}$ is in better (but still poor) agreement with the observed signal variability.

The value of $T_{A, A t m}$ at 7.5 can be compared to measurements from Barcroft at nearby frequencies. Results from 3.8, 7.5, and $10.0 \mathrm{GHz}$ are summarized in Table 4.15. The model of atmospheric emission predicts $\mathrm{T}_{\mathrm{A}, \mathrm{Atm}}$ at $7.5 \mathrm{GHz}$ to be smaller than at $10 \mathrm{GHz}$ by $100-150 \mathrm{mK}$, and larger than $3.8 \mathrm{GHz}$ by $80-130 \mathrm{mK}$. The observed differences were 95 and $150 \mathrm{mK}$, respectively. The difference (although not the absolute magnitudes) from 3.8 to $10 \mathrm{GHz}$ was also in good agreement with the model, suggesting that the value for $T_{A, A t m}$ at $7.5 \mathrm{GHz}$ was high by $\sim 30 \mathrm{mK}$. This is well within the uncertainty of the measurements. There is no evidence for undetected systematics in the atmospheric measurements at $7.5 \mathrm{GHz}$. One is led to the conclusion that, at frequencies below $10 \mathrm{GHz}$, the data are better understood than the aumospheric model.

\subsubsection{The CMB}

Table 4.16 lists recent low-frequency CMB measurements below $40 \mathrm{GHz}$. Assuming the uncertainties are independent and follow gaussian statistics, the average of the measurements weighted by the uncertainties is a valid indicator of the mean of the parent distribution (assuming, of course, that no CMB distortions are present). The weighted mean of all measurements below $40 \mathrm{GHz}$, excluding the $7.5 \mathrm{GHz}$ datum, is $2.748 \pm 0.021 \mathrm{~K}\left(\chi^{2}=10.4 / 7 \mathrm{DOF}\right.$, corresponding to a confidence level 0.2$)$. The result at $7.5 \mathrm{GHz}, 2.59 \pm 0.07 \mathrm{~K}$, is 2.2 standard deviations away. The mean is heavily weighted by the balloon-borne measurement at $24.8 \mathrm{GHz}$, which was indeed intended as a benchmark. Most of the contribution to the $\chi^{2}$ results from this measurement and the next most precise measurement at $10 \mathrm{GHz}$. If we arbitrarily reverse the roles of the 7.5 and $24.8 \mathrm{GHz}$ points and calculate the weighted mean including the $7.5 \mathrm{GHz}$ result and excluding the $24.8 \mathrm{GHz}$ result, the mean $\mathrm{CMB}$ temperature below $40 \mathrm{GHz}$ is $2.653 \pm 0.033 \mathrm{~K}\left(\chi^{2}=5.2 / 7 \mathrm{DOF}, \mathrm{C} . \mathrm{L} .=0.65\right)$. The measurement at $7.5 \mathrm{GHz}$ is in excellent agreement with ground-based measurements, which in turn are in poor agreement with the higher-frequency balloon-borne measurement at $24.8 \mathrm{GHz}$.

In fact, the ground-based measurements are not completely independent, and share some fraction of the total systematic uncertainty (e.g., use of a common cold load). In this case, the estimated uncertainty of $0.033 \mathrm{~K}$ for all ground-based results will be an 
underestimate. The basic conclusion, however, remains unchanges: that the ground-based results are in conflict with measurements at higher frequencies using different techiniques. This point will be addressed in greater detail in Chapter 6 . 


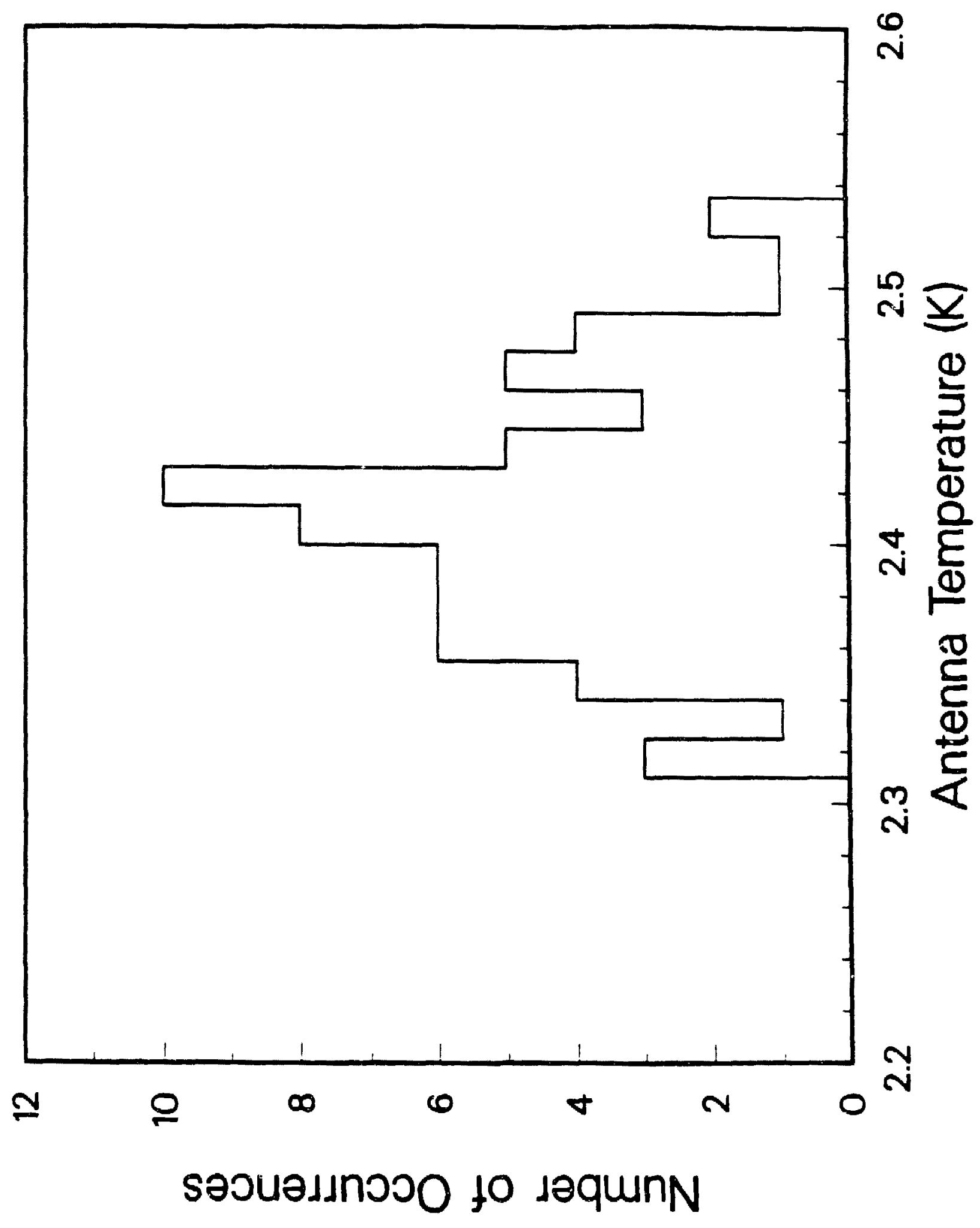

Figure 4.7: Histogram of the 65 independent determinations of $T_{A, C M B}$. 


\section{Chapter 5 \\ Remote Measurement at $141 \mathrm{GHz}$}

\subsection{Introduction}

A cosmological origin of the $\mathrm{CMB}$ makes three firm and testable predictions: the CMB should have a Planckian spectrum, should be the same throughout the local universe, and should increase with redshift $z$ as $T(z)=T_{0}(1+z)$. In this chapter, I shall discuss the results of an experiment that could test the latter prediction.

Interstellar spectroscopy provides a method to determine local conditions at remote locations. While we cannot launch probes throughout the galaxy or back in time to earlier epochs in the lniverse, we can detect the photons which have made the journey and which carry the signature of their interactions with the intervening matter. Typically, one observes a set of molecular absorption lines arising from a common quantum state, seen in cold molecular clouds in front of bright continuum sources. From the relative strengths of the lines and a knowledge of molecular physics, the observer can deduce the thermal background in the absorbing clouds.

This technique has been used successfully with the cyanogen molecule (CN). The energy levels involved are shown in Figure 5.1. The low-lying rotational levels have transitions (among others) at 2.64 and $1.32 \mathrm{~mm}$, which are well-populated by the intense $\mathrm{CMB}$ at $\mathrm{mm}$ wavelengths. The relative populations in the rotational levels can be determined by observations of electronic transitions in the optical between these levels and levels in a higher band. The source of photons at $3874 \AA$ is a bright star. Recent results from CN absorption toward $\zeta$ Oph yield a CMB temperature of $2.796_{-0.041}^{+0.019}$ (Crane et al. 1988).

The line widths of the observed $C N$ transitions are a few $\mathrm{km} \mathrm{s}^{-1}$, below the instrumental resolution of the optical spectrometers used. The resultant correction is largely responsible for the recent upward change in reported $C N$ results with time, from 2.70 \pm 0.04 (Meyer and Jura 1985) to $2.796_{-0.041}^{+0.019}$ (Crane et al. 1988). A more serious constraint is the required illuminating star. Although $\mathrm{CN}$ indicates that the $\mathrm{CMB}$ at locations throughout our galaxy has the same brightness as measured on Earth, individual stars cannot be resolved at redshifts of cosmological interest (z !). CN is unlikely to produce an extragalactic CMB measurement. 


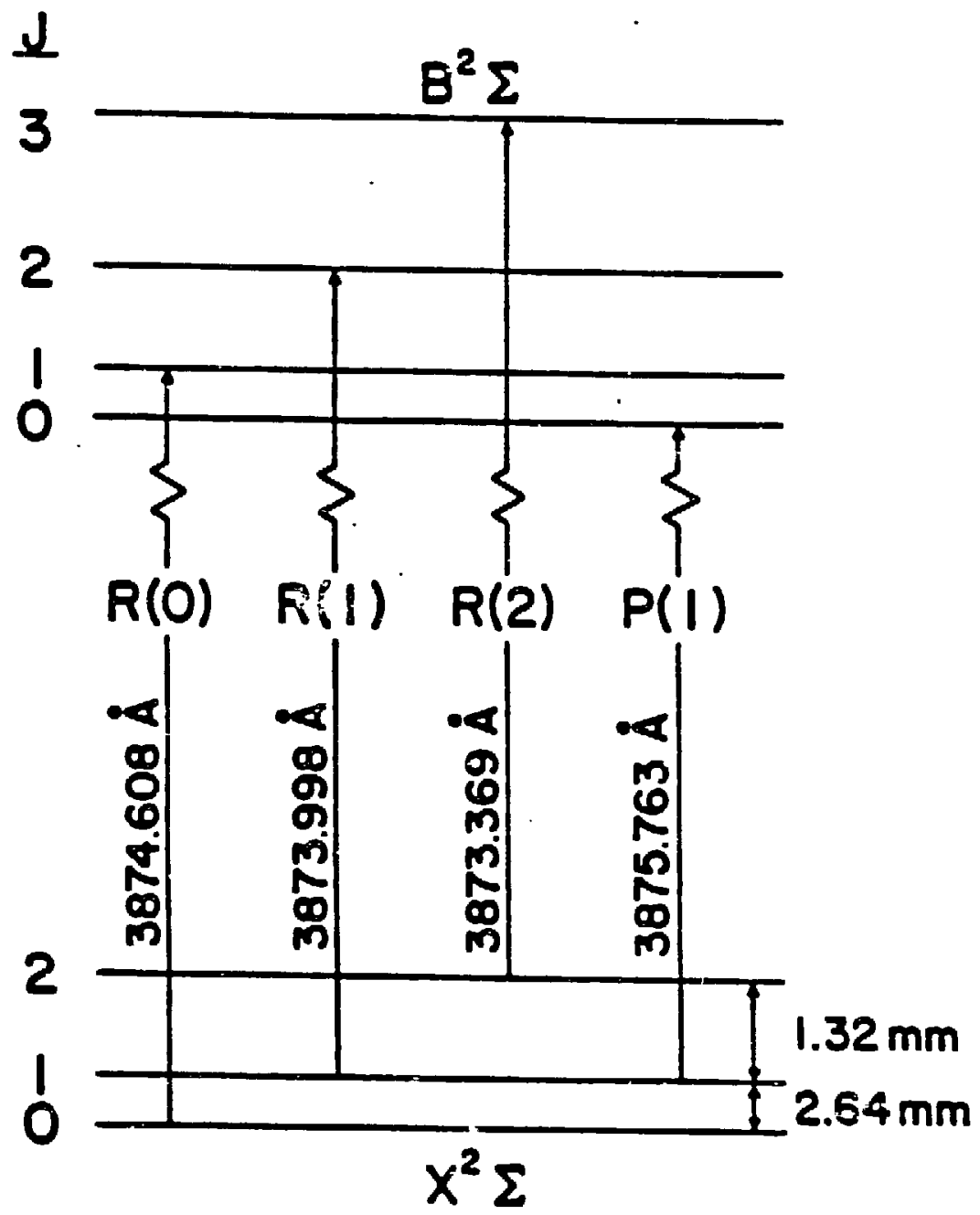

Figure 5.1: Transitions in cyanogen used to determine the CMB temperature. 
One possibility for extragalactic $\mathrm{CMB}$ measurements lies in atomic spectra. Optical fine-structure transitions in the ground state of neutral carbon ( $\mathrm{C}^{0}$ or $\mathrm{C}$ I) are sensitive to the $\mathrm{CMB}$ at a wavelength of $0.61 \mathrm{Im}$, corresponding to an energy gap of $23.6 \mathrm{~K}$. A search for these lines in QSO spectra resulted in a weak upper limit $\mathrm{T}_{\mathrm{CMB}}<16 \mathrm{~K}$ at a redshift $\mathrm{z}=1.776$ (Meyer et al. 1986).

In 1985 George Smoot, Chuck Bennett, Sam Petuchowski and I began to explore the possibility of using interstellar formaldehyde $\left(\mathrm{H}_{2} \mathrm{CO}\right)$ to provide a remote detection of the $\mathrm{CMB}$ intensity at $2.1 \mathrm{~mm}(140.8 \mathrm{GHz})$. Formaldehyde has several observational advantages over $\mathrm{CN}$ or $\mathrm{CI}$. The observed transitions are between rotational states and lie at microwave frequencies. Modern radio telescopes can easily resolve lines with widths of a few $\mathrm{km} \mathrm{s}^{-1}$. The illuminating continuum sources are $\mathrm{HIl}$ regions, extending over several minutes of arc; the observer is thus not limited to a single line of sight but can map the absorption and CMB temperature. The association of large HII regions and absorbing clouds makes $\mathrm{H}_{2} \mathrm{CO}$ a good candidate for detection at mocierate redshifts.

\subsection{Concept of the Experiment}

\subsubsection{Molecular Parameters}

The lower-lying rotational energy levels of ortho-formaldehyde are shown in Figure 5.2, with allowed transitions between the states. $\mathrm{H}_{2} \mathrm{CO}$ is a slightly asymmetric prolate rotor whose rotational energy levels show considerably more complicated structure than those of a simple linear molecule. The rotational levels of total angular momentum $\mathrm{J}$ are split by the slight asymmetry along the $B$ and $C$ axes into doublets with $\Delta J=0, \Delta K= \pm 1$ transitions between them. The relative populations of the levels are determined by detailed balance between:

- The rates of spontaneous and induced radiative transitions in the local radiation field

- The rates of non-radiative processes allowing transitions between levels (principally collisional excitation from neutral hydrogen molecules $\left[\mathrm{H}_{2}\right]$ ).

- The photon escape probability, determined by the geometry of the absorbing clouds. Observations of the doublet transitions in the $\mathrm{J}=1$ and $\mathrm{J}=2$ levels determine the relative populations of the $2_{12}$ and $1_{11}$ levels, which are connected by a $\Delta \mathrm{J}= \pm 1 \Delta \mathrm{K}=0$ radiative transition at rest frequency $140.8 \mathrm{GHz}(2.1 \mathrm{~mm})$.

The molecular parameters of ortho- $\mathrm{H}_{2} \mathrm{CO}$ are summarized in Table 5.1. 


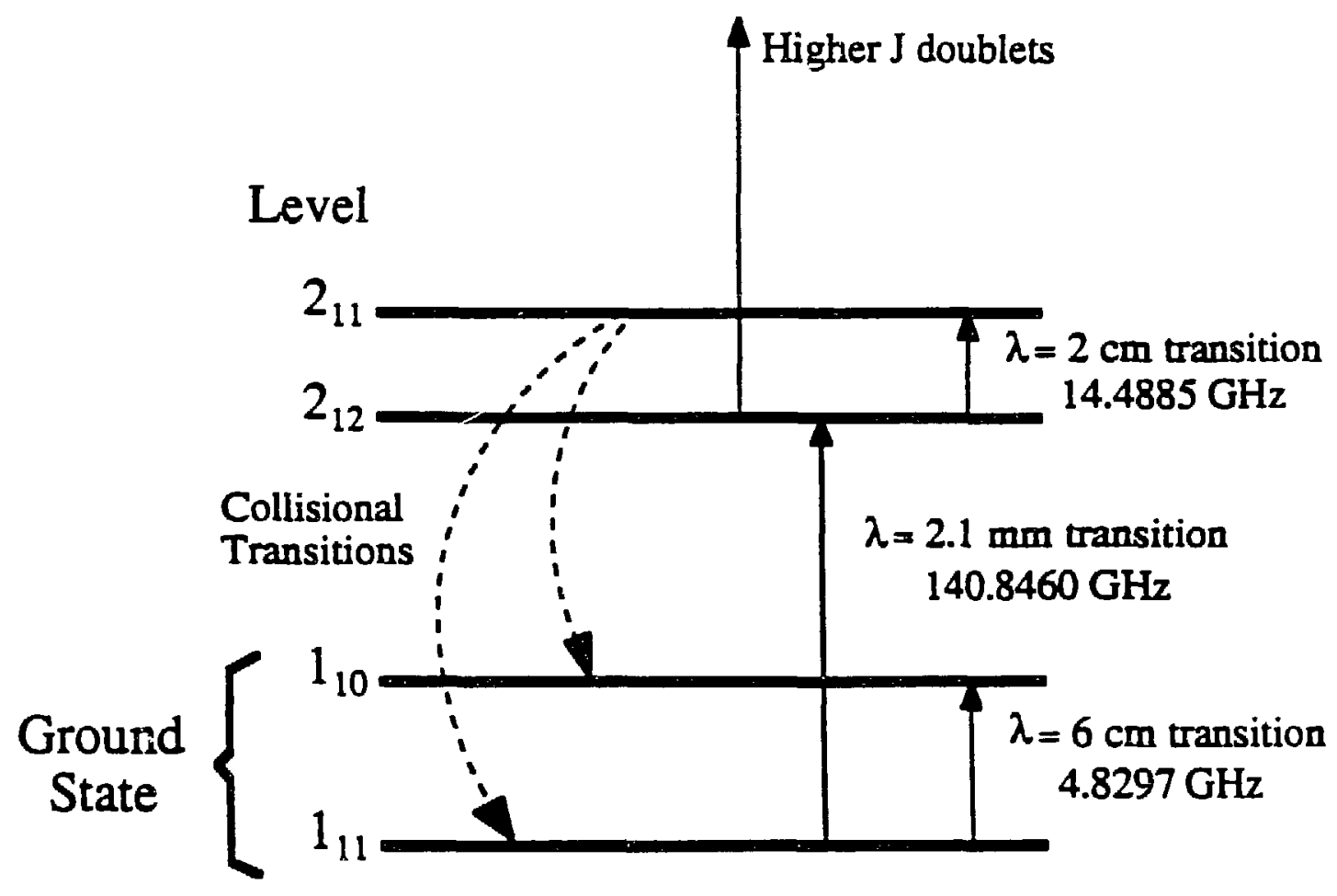

Figure 5.2: Rotational energy levels of ortho-formaldehyde. 


\subsubsection{Anomalous Absorption and Pumping}

The largest correction to a purely radiative scheme is collisional excitation from $\mathrm{H}_{2}$. $\mathrm{H}_{2} \mathrm{CO}$ is of ten seen in absorption in the absence of any discrete continuum source. Some mechanism pumps the $\lambda=6 \mathrm{~cm}$ and $2 \mathrm{~cm}$ doublet transitions to subthermal values so that they absorb against the reservoir of $\mathrm{CMB}$ photons. A number of mechanisms were proposed to explain this, of which $\mathrm{H}_{2}$ collisional pumping and sharp spectral features in the CMB at $2.1 \mathrm{~mm}$ were the leading candidates (Thaddeus 1972 and references therein). The theoretical activity surrounding $\mathrm{H}_{2} \mathrm{CO}$ and anomalous absorption and its ubiquity and ease of observation make this molecule one of the best-studied under temperatures and densities typical of interstellar conditions.

Deviations from a Planck spectrum at the $\Delta \mathrm{J}=1$ transition frequencies can pump the doublet transitions to subthermal values. If the CMB is hotter at the frequency of the $211^{-}$ $1_{10}$ transition $(150 \mathrm{GHz})$ than at the frequency of the $22^{-1} 1_{11}$ transition $(141 \mathrm{GHz})$, it will effectively overpopulate the $2_{11}$ level at the expense of the $1_{10}$ level. The $1_{10}$ level is then underpopulated with respect to the $\mathrm{CMB}$ temperature at the frequency of the $\Delta \mathrm{J}=0$ doublet transition, and will appear in absorption against the CMB. The amount of pumping depends on the magnitude of the spectral deviation at $\mathrm{mm}$ wavelengths and the rates of radiative transitions among the lower levels of formaldehyde. If all levels past $\mathrm{J}=\mathbf{2}$ are ignored, the lowest four levels are related by (Evans et al. 1975)

$$
T_{34}=\varepsilon_{34}\left\{\frac{\varepsilon_{12}}{T_{12}}+\frac{\varepsilon_{24}}{T_{24}}-\frac{\varepsilon_{13}}{T_{13}}\right\}^{-1}
$$

where $T_{i j}$ is the excitation temperature characteristic of the transition between levels $i$ and $j$, $\varepsilon_{i j}$ is the reduced energy difference $h v_{i j} / k$, and the levels have been labelled consecutively from the lowest level $1_{11}$. An excess $\Delta \mathrm{T}$ at $150 \mathrm{GHz}$ relative to $141 \mathrm{GHz}\left(\mathrm{T}_{24}>\mathrm{T}_{13}\right)$ will then produce a cooling in the lower $6 \mathrm{~cm}$ doublet. The doublet absorption line depths of $-1 \mathrm{~K}$ at $6 \mathrm{~cm}$ could be explained by spectral distortions of $-0.15 \mathrm{~K}$ at $2 \mathrm{~mm}$ wavelength.

This simple model has problems. Interstellar formaldehyde, although optically thin at $\mathrm{cm}$ wavelengths, is optically thick in the $\mathrm{mm}$ transitions. When the effects of $\mathrm{mm}$ photon trapping are included, the magnitude of the spectral pumping is greatly reduced. The spectral model also predicts that sub-thermal cooling in one of the doublet transitions should be accompanied by heating of the other doublet transition. Observations of both doublet transitions in anomalous absorption show that both appear in absorption against une 
CMB (Evans et al. 1975). CMB spectral distortions alone cannot explain the relative level populations of interstellar $\mathrm{H}_{2} \mathrm{CO}$, although they may be present at some level.

An altemative explanation is that non-radiative transitions alter the relative populations of the lower-lying levels. A series of numerical quantum-mechanical calculations showed that collisions with hydrogen could produce the observed cooling in both the 6- and 2-cm transitions under conditions typical of molecular clouds (Townes and Cheung 1969, Garrison et ai. 1975, Green et al. 1978). Resonances in the $\mathrm{H}_{2} \mathrm{CO}-\mathrm{H}_{2}$ system preferentially pump both doublet transitions to sub-thermal values for $\mathrm{H}_{2}$ densities below $\mathrm{n}\left(\mathrm{H}_{2}\right)-10^{6} \mathrm{~cm}^{-3}$, depending slightly on the kinetic temperature of the $\mathrm{H}_{2}$ (and hence the collision rate). The collisional pumping depends on transitions to higher rotational levels of $\mathrm{H} 2 \mathrm{CO}$; if the system is modelled with only the $\mathrm{J}=1$ and $\mathrm{J}=2$ levels, the pumping disappears.

\subsubsection{Non-LTE Radiative Transfer}

Formaldehyde in interstellar molecular clouds is not in a state of local thermodynamic equilibrium (LTE), and cannot be characterized by a single temperature. Statistical equilibrium for the level populations $n_{i}$ yields the equation

$$
\begin{aligned}
0=\frac{d N_{i}}{d t}=\sum_{j \neq i}\left\{\left[\beta_{v}\left(A_{j i}+B_{j i} u_{v}\right)+n\left(H_{2}\right) \gamma_{j i}\right] n_{j}\right. \\
\left.-\left[\beta_{v}\left(A_{i j}+B_{i j} u_{v}\right)+n\left(H_{2}\right) \gamma_{i j}\right] n_{i}\right\}
\end{aligned}
$$

where $A_{i j}$ and $B_{i j}$ are the Einstein $A$ and $B$ coefficients for transitions between levels $i$ and $j$ of ortho-formaldehyde; $u_{v}$ is the energy density of the local radiation field at the frequency $v$ of the transition; $\gamma_{i j}$ is the rate of collisional excitation; $n\left(H_{2}\right)$ is the space density of molecular hydrogen, and $\beta_{v}$ is the photon escape probability per transition. Throughout this chapter I shall use the convention that higher-numbered states are higher in energy; hence, $A_{i j} \neq 0$ only for $i<j$ (spontaneous emission). Detailed balance provides a relation between the upward and downward transitions:

$$
\begin{gathered}
g_{i} B_{i j}=g_{j} B_{j i} \\
g_{i} \gamma_{i j}=g_{j} \gamma_{j i} \exp \left(\frac{h v_{i j}}{k T_{k i n}}\right)
\end{gathered}
$$


where $g_{i}$ and $g_{j}$ are the statistical weights of levels $i$ and $j, h$ is Planck's constant, and $T_{k i n}$ is the kinetic temperature of the molecular hydrogen. The collisional excitation rates themselves have a temperature dependence,

$$
\gamma_{\mathrm{ij}}=\gamma_{\mathrm{ij}}\left(\mathrm{T}_{\mathbf{k i n}}\right)
$$

which must be included in detailed calculations of level populations. The local radiation field is assumed to be a combination of a blackbody at temperature $T_{C M B}$,

$$
u_{v}=\frac{A_{i i}}{B_{i j}}\left[\exp \left(\frac{h v}{k T_{C M B}}\right)-1\right]-1 \text {, }
$$

and greybody emission from warm dust (discussed in \$5.5.3). For observations limited to the absorption lines of $\mathrm{H}_{2} \mathrm{CO}$, the only observables in Eq. 5.3 are contained in the photon escape probability, $\beta_{v}$. The escape probability is a function of the local geometry and kinematics and the opacity $\tau$, where for an isotropic homogeneous medium the opacity $\tau$ is defined by the equation of radiative transfer

$$
\begin{aligned}
& \tau_{v}=\int_{0}^{s} \alpha_{v}\left(s^{\prime}\right) d s^{\prime} \\
& \frac{d I_{v}}{d \tau_{v}}=-I_{v}+S_{v} .
\end{aligned}
$$

Here $\alpha_{v}$ is the absorption coefficient $\left(\mathrm{cm}^{-1}\right), I_{v}$ is the specific intensity of the continuum, $S_{V}$ is the source function of the cloud, and $s$ the path length. For an arbitrary geometry, Eq. 5.8 may have no simple analytic solutions.

The observed line widths in molecular clouds are a few $\mathrm{km} \mathrm{s}^{-1}$, much greater than the thermal widths of order $0.1 \mathrm{~km} \mathrm{~s}^{-1}$ expected for molecules in cold $(10-50 \mathrm{~K})$ clouds. In the absence of turbulent shocks in the clouds, the widths imply large-scale velocity gradients in the molecular clouds. Thermal pressure inferred from even the strongest molecular emission lines $(-70 \mathrm{~K})$ is insufficient to support the clouds against gravitational collapse. The clouds are usually modelled as pressure-free spheres of constant density with radial velocities proportional to the distance $r$ from cloud center,

$$
v(r)=r\left(\frac{2 G M}{R^{3}}\right)^{1 / 2}=\frac{\Delta V}{R} r
$$


where $\mathrm{M}$ is the total cloud mass and $\mathrm{R}$ is the outer cloud radius. The Large Velocity Gradient ( $L V G$ ) model reduces the problem of radiative transport to a local one, in that photons emitted at radius $r$ interact only within a distance $\delta r$ given by the thermal width $v_{\text {thermal, }}$

$$
\delta r-R \frac{y_{\text {thermal }}}{\Delta V}
$$

not over the entire radius $R$ of the cloud. The opacity $\tau_{i j}$ is then given in terms of the populations $n_{i}, n_{j}$, and the velocity gradient $d v / d r$ by

$$
\tau_{i j}=\frac{h}{d v / d r}\left\{n_{i} B_{i j}-n_{j} B_{j i}\right\}
$$

(Lucy 1971). For this spherical geometry the photon escape probability $\beta_{v}$ is

$$
\beta_{v}=\frac{1-\exp \left(-\tau_{\mathrm{ij}}\right)}{\tau_{\mathrm{ij}}}
$$

(Goldreich and $\mathrm{Kwan}$ 1974). Given the observed opacities $\tau_{\mathrm{ij}}$ in the doublet transitions at 6- and 2- cm wavelength, Eqs. 5.3, 5.10, and $5.11 \mathrm{can}$ be solved iteratively for the CMB temperature and the hydrogen density, provided enough levels are used in the model that transitions from the uppermost levels contribute only slightly to the total populations in the lower levels.

\subsubsection{Observing Strategy}

The ratio of opacities $\tau_{6 \mathrm{~cm}} / \tau_{2 \mathrm{~cm}}$ in the 6 - and 2 - $\mathrm{cm}$ doublet transitions determines the relative populations of the $2_{12}$ and $1_{11}$ rotational levels. Interpretation of the ratio is complicated if substantial unresolved structure exists in the clouds. To reduce this problem, we require observations of the same high angular resolution at both 6 and $2 \mathrm{~cm}$ wavelength. This minimizes effects of opacity gradients within a resolution element, and removes them to second order in the ratio of the opacities.

To minimize effects of collisional pumping, we desire a source with low $\mathrm{H}_{2}$ density, and hence optically thin lines of $\mathrm{H}_{2} \mathrm{CO}$. The simultaneous high sensitivity and identical high angular resolution needed to detect weak lines in highly-structured clouds led us to the 
NRAO'sa Very Large Array (VLA). The VLA is an interferometer consisting of $2725-\mathrm{m}$ telescopes operated in several wavelength-scaled configurations. By observing the 6-cm transition in the VLA C configuration and the 2-cm transition in the D configuration, the resultant synthesized beamwidth (4") was nearly identical at the two wavelengths. Appendix $\mathrm{C}$ contains a brief review of interferometry.

The source observed was a compromise between systematic corrections for collisional pumping (suggesting optically thin lines) and telescope integration time (suggesting strong absorption lines). To ensure that statistical noise in the 2-cm absorption maps would not be a limiting factor, we chose a source with bright continuum radiation and moderate opacities in the foreground absorbing clouds, at the cost of some complexity in the source and cloud structure. W51A (G49.5-0.4) is a giant $\mathrm{HII}$ region located in the Sagittarius spiral arm at a distance of approximately $7.5 \mathrm{kpc}$ (Genzel et al. 1981). W51 is near the tangent point of the spiral arm. The line of sight traverses some $5 \mathrm{kpc}$ of spiral arm, with consequent projection effects. Aidhough the large path length complicates the morphology of the observed structures, it also allows observation of foreground clouds not directly associated with the warm cores of the HII regions. The absorbing clouds toward W51 had been mapped in the 6-cm transition of formaldehyde at 12" angular resolution, and seemed to contain regions suitable for more detailed analysis (Marin-Pintado et al. 1985). In particular, a strong $\mathrm{H}_{2} \mathrm{CO}$ absorption line between $64-67 \mathrm{~km} \mathrm{~s}^{-1}$ existed, which had been identified with foreground molecular clouds (Amal and Goss 1985, Martin-Pintado et al. 1985). We did not attempt to model absorption toward the massive core of W51, restricting analysis instead to the coider, less dense foreground clouds.

\subsection{Observations and Data Reduction}

\subsubsection{Transition at $6 \mathrm{~cm}$}

We observed the 6 -cm transition (rest frequency $4.8296596 \mathrm{GHz}$ ) on 1986 Nov 8 using the VLA in C configuration. Spectral coverage consisted of the inner 32 channels of

\footnotetext{
a The National Radio Astronomy Observatory is operated by Associated Universities, Inc., under contract with the National Science Foundation.
} 
a 256-channel, $3.125-\mathrm{MHz}$ bandpass centered at $65.0 \mathrm{~km} \mathrm{~s}^{-1} \mathrm{LSR}^{\mathrm{b}}$; the velocity resolution was $0.76 \mathrm{~km} \mathrm{~s}^{-1}$. A total of 5.5 hours was spent on source. We calibrated the flux scale using an assumed value of $7.46 \mathrm{Jy}$ for $3 \mathrm{C} 286$, and used a $0.5 \mathrm{hr}$ integration of $3 \mathrm{C} 84$ to calibrate the bandpass. Phase calibration used $1923+210$, observed for 5 minutes of every 30. The VLA's response is improved if the phase center of observations is not coincident with a bright source; consequently, we displaced the phase center 1 ' west of the brightest continuum.

\subsubsection{Transition at $2 \mathrm{~cm}$}

We observed the $2 \mathrm{~cm}$ transition (rest frequency $14.488479 \mathrm{GHz}$ ) on 1987 May 1,2 , and 3, with the VLA in the D configuration. Spectral coverage consisted of the inner 32 channels of a 128-channel, 3.125-MHz bandpass, again centered at $65.0 \mathrm{~km} \mathrm{~s}^{-1} \mathrm{LSR}$, for velocity resolution of $0.51 \mathrm{~km} \mathrm{~s}^{-1}$. A total of 14.5 hours was spent on source, slightly less than expected owing to a power failure at the VLA site on May 3. We calibrated the flux scale using a value for $3 \mathrm{C} 286$ of $3.53 \mathrm{Jy}$. Accurate absolute measurement of the opacity at $2 \mathrm{~cm}$ required an accurate determination of the bandpass calibration. We used a $1.5 \mathrm{hr}$ integration of $3 \mathrm{C} 84$ ( $0.5 \mathrm{hrs}$ at the end of each of the 3 observing runs) for bandpass calibration, ensuring that bandpass calibration was not a limiting error in our determination of the absorption spectra. As with the $6 \mathrm{~cm}$ observations, we used $1923+210$, observed 5 minuiws of every 30 , for phase calibration. The phase center was placed closer to the continuum peak W5le to ensure that the regions of greatest interest (W51e and W51d) lay inside the half-power point of the primary antenna beam. Table 5.2 lists observing parameters for both the $6 \mathrm{~cm}$ and $2 \mathrm{~cm}$ observations.

\subsubsection{Data Reduction}

We employed standard VLA calibration and data reduction software to convert the observed visibilities to intensity maps. The three days of $2 \mathrm{~cm}$ observation were calibrated independently before being combined into a single visibility database. The dirty maps were CLEANed and the CLEAN components used as input for self-calibration of the phases.

For either frequency, Channel Zero contained the average of the inner three-cuarters of the originally available passband. The velocity range covered in the 31 spectral channels

\footnotetext{
$\mathrm{b}$ The standard radio definition of velocity is $\Delta v=\frac{v-v_{C}}{v_{0}} c$, where $v$ is the observed line frequency and $v_{0}$ the rest frequency; $c$ is the velocity of light. All frequencies are corrected to the local standard of rest (LSR).
} 


\begin{tabular}{ll}
\hline$A_{0}$ & $282106 \mathrm{MHz}$ \\
$B_{0}$ & $38834 \mathrm{MHz}$ \\
$\mathrm{C}_{0}$ & $34004 \mathrm{MHz}$ \\
$\mu$ & $2.34 \times 10^{-18}$ esu cm \\
\hline
\end{tabular}

Table 5.1: Molecular parameters of ortho-formaldehyde.

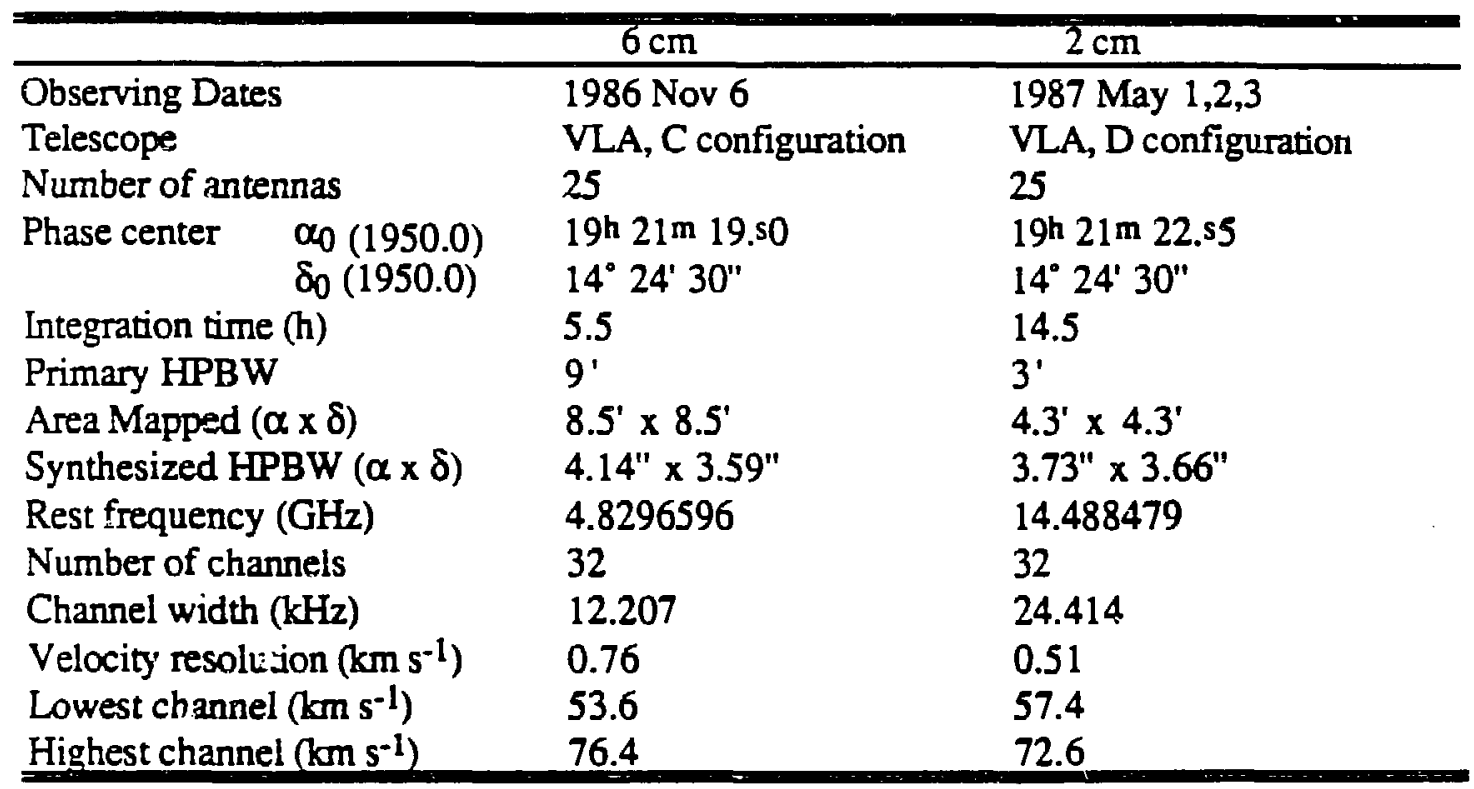

Table 5.2: Observing Parameters at 6 and $2 \mathrm{~cm}$.

\begin{tabular}{lrr}
\hline Feature & \multicolumn{2}{c}{ Peak Intensity $(\mathrm{mJy} / \mathrm{beam})$} \\
& $6 \mathrm{~cm}$ & $2 \mathrm{~cm}$ \\
\hline W51d & $1223 \pm 16$ & $1970 \pm 29$ \\
W51e & $779 \pm 25$ & $830 \pm 31$ \\
W51b & $198 \pm 5$ & $162 \pm 3$ \\
W51 $_{1}$ & $125 \pm 9$ & $155 \pm 6$ \\
W51e2 $_{2}$ & $48 \pm 4$ & $177 \pm 6$ \\
\hline
\end{tabular}

Table 5.3: Peak intensity of the brighter continuum regions. Estimated uncertainties are statistical only. 
had significant absorption in most channels; these channels formed a non-negligible fraction (24\%) of Channel Zero. Spectra over a wider velocity range (Bieging et al. 1982) show no absorption features which would have contributed significantly to our Channel Zero that would not be seen in our 31 spectral channels. Accordingly, we formed a continuum map at both 6- and 2- $\mathrm{cm}$ by subtracting from Channel Zern the suitably weighted average of the 31 data channels. We then aypiied a correction for the primary beam pattern, a significant effect at $2 \mathrm{~cm}$ where the primary FWHM was approximately the size of the area of interest.

As with any interferometer, the VLA's sensitivity to large-scale structure is determined by the shortest baselines available and the details of the source structure. W51 is known to have considerable extended emission surrounding the compact sources (Bieging et al. 1982, Gardner and Whiteoak, 1984). Our continuum maps contained $48 \%$ and $55 \%$, respectively, of the flux observed by single-dish measurements at $6-$ and $2-\mathrm{cm}$ wavelength. We added the "missing" flux as a constant across the continuum maps; this amounted to $9.8 \mathrm{mJy} /$ beam at $6 \mathrm{~cm}$ and $17.8 \mathrm{mJy} / \mathrm{beam}$ at $2 \mathrm{~cm}$, effectively raising the zero level of the maps without changing the noise properties. The resultant continuum maps have an RMS noise of $1.5 \mathrm{mJy} / \mathrm{beam}$ at $6 \mathrm{~cm}$, and $3 \mathrm{mJy} / \mathrm{beam}$ at $2 \mathrm{~cm}$, in blank fields near the phase center. The RMS noise rises with increasing distance from the phase center in accordance with the primary beam pattern (Table 5.2).

We produced dirty maps for each spectral channel, as well as a dirty continuum map (without tiac corrections described above). All maps were 512" $\times 512$ " $(\alpha \times \delta)$ at $6 \mathrm{~cm}$ and $256 " \times 256 "$ at $2 \mathrm{~cm}$, with uniform weighting at both wavelengths to maximize resolution. The absorption maps are the differences between the dirty channel maps and the dirty continuum, CLEANed and corrected for the primary beam pattern. The absorption maps have RMS noise near the phase center of $4 \mathrm{mJy} / \mathrm{beam}$ at $6 \mathrm{~cm}$ and $7 \mathrm{mJy} / \mathrm{beam}$ at $2 \mathrm{~cm}$. We then made maps of the optical depth of the formaldehyde lines in the usual fashion, with the observed opacity $\tau$ is defined as

$$
\tau=-\ln \left(1-\frac{\Delta T_{L}}{T_{c}}\right)
$$

where $\Delta T_{L}$ is the antenna temperature difference at line center between the continuum and the absorption line.

To prevent systematic uncertainties in the subtraction of the continuum from affecting the results, we blanked the optical depth maps wherever corrections to the 


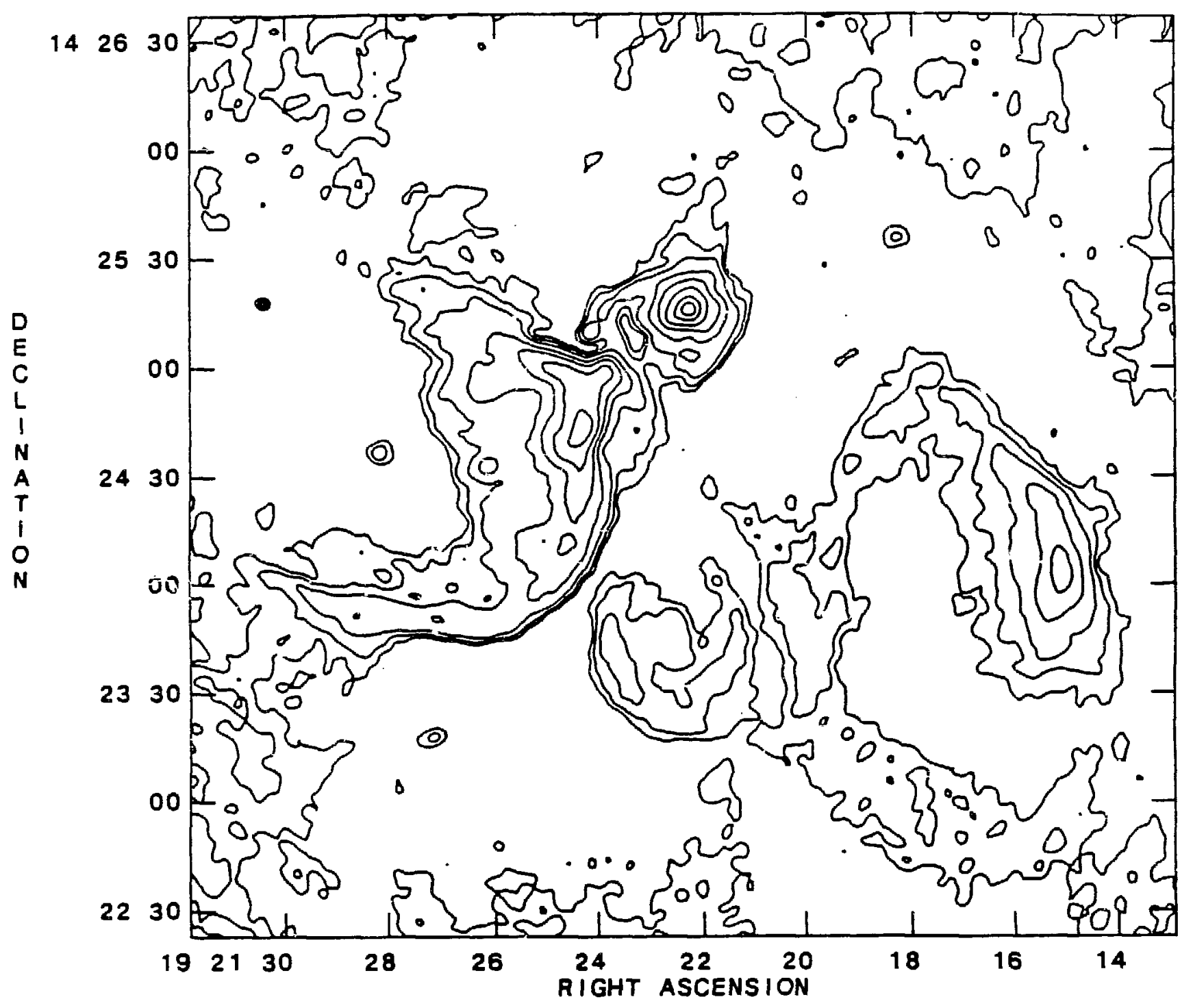

Figure 5.3a: $6-\mathrm{cm}$ continuum toward W51A. The map has been CLEANed and corrected for primary beam pattern. Contours are at 10,20,40,100,200,400,800, and $1200 \mathrm{mJy} /$ beam. The beam size and shape are indicated by the black oval to the upper left. 


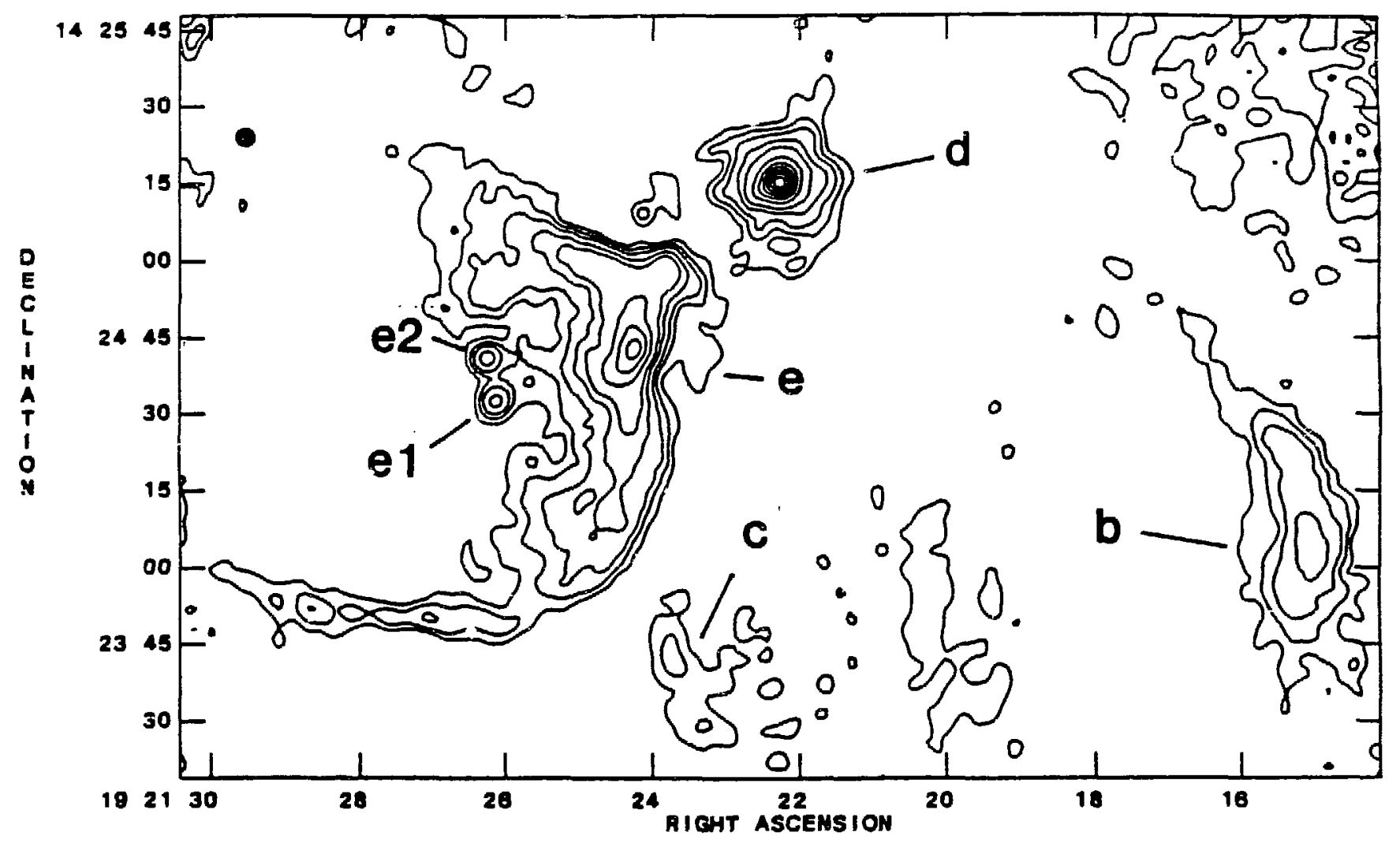

Figure 5.3b: 2-crn continuum. The first six contours are at 30,50,80,150, 250, and $500 \mathrm{mJy} / \mathrm{beam}$; successive contours are spaced at $300 \mathrm{mJy} /$ beam intervals. The designations of Martin (1972) are indicated. 
continuum for missing flux were more than $10 \%$ of the continuum. The cutoff level is 100 $\mathrm{mJy} /$ beam for the $6 \mathrm{~cm}$ continuum and $75 \mathrm{mJy} / \mathrm{beam}$ at $2 \mathrm{~cm}$. The maximum error induced in the opacity maps by zero-spacing flux uncertainty is $10 \%$ at the weakest continuum and falls to $<1 \%$ at the continuum peaks. As the continuum flux levels and the response of the array are similar at both 6 and $2 \mathrm{~cm}$, instrumental errors largely cancel; the systematic uncertainty in the ratio of the opacities is smaller than the uncertainty in either of the individual measurements.

Figure 5.3 shows the continuum intensity of W51A as a function of position on the sky, for both the $6-\mathrm{cm}$ and $2-\mathrm{cm}$ observations. The beam shape $(\approx 4 ")$ is indicated by the black oval on each plot. The brighter continuum peaks are labelled according to the notation of Martin (1972) and Scott (1978). The extended diffuse continuum emission is visible at the edges of the maps, where it falls near the first contour. RMS noise, magnified by the correction for prinary beam pattern, is also visible in the comers of the 2$\mathrm{cm}$ continuum plot. The similar flux at both wavelengths is characteristic of $\mathrm{HI}$ regions. Table 5.3 lists the peak intensities of the brighter continuum peaks. Although we observed absorption over most of the continuum features, we retained for analysis only those regions with continuum flux density greater than the cutoff $(100 \mathrm{mJy} / \mathrm{beam}$ at $6 \mathrm{~cm}, 75 \mathrm{mJy} / \mathrm{beam}$ at $2 \mathrm{~cm}$ ).

The $\mathrm{H}_{2} \mathrm{CO}$ absorption, converted to opacity plots, shows a complicated morphology. Figures 5.4 and 5.5 show contour plots of the opacity in selected velocity channels for both the 6- and 2-cm transitions. A number of features are obvious. The molecular clouds are dominated by clumping on scales down to the beam width, corresponding to linear scales of $0.15 \mathrm{pc}$. The opacity increases to the E of W5le and to the N and NW of W51d, in qualitative agreement with observations of lower angular resolution (Amal and Goss 1985; Marin-Pintado et al. 1985). In general, we derive higher opacities in the clump peaks, as would be expected for our finer angular resolution in the presence of small-scale clumping. A notable new feature of the maps is the concentration of the largest opacities into extended, ridged features, particularly near 66 and $69.5 \mathrm{~km} \mathrm{~s}^{-1}$ toward W51e. The opacity enhancements of these features is large; at $69 \mathrm{~km} \mathrm{~s}^{-1}$, the 6-cm opacity varies from 0.71 to 0.15 in a single beamwidth. The linear extent of these features is also large, at least $1.5 \mathrm{pc}$ (the extent of the illuminating continuum).

The spectra are also complicated. Figure 5.6 shows the spectra (at $6 \mathrm{~cm}$ ) as a function of position across W51e and W5Id. The spectra are broad (FWHM typically 2$4 \mathrm{~km} \mathrm{~s}^{-1}$ ) and contribute substantial signal to several channels to either side of the spectral 
peak. Toward the W51d and the southem half of W51e, a given pixel at map coordinates $(\alpha, \delta, V)$ will contain superposed contributions from absorption features at nearby positions and velocities. To adequately model radiative transfer within a single cloud condensation ("clump"), the contribution from single clumps must be isolated and parametrized.

The signal-to-noise ratio $(\mathrm{S} / \mathrm{N})$ of our data allowed such deconvolution of the spatial and velocity structure. For each $(\alpha, \delta)$ pixel in our channel maps, we modelled the velocity spectrum as consisting of either two, one, or no superposed gaussian profiles. Although the noise in the absorption and continuum maps are known and fairly constant, the changing continuum magnitude substantially alters the noise as a function of position in the opacity spectra (Eq. 5.12). Signal fluctuations in several "blank" channels at both the high- and low-velocity ends of the spectrum served to estimate the noise at each position The resultant $\chi^{2}$ of the model determined the number of gaussian profiles at each $(\alpha, \delta)$ pixel. We rejected modelled profiles with unphysical parameters (e.g., FWMH less than 0.5 channel width or greater than $7 \mathrm{~km} \mathrm{~s}^{-1}$ ) and those whose velocity centroids were outside the velocity range sampled. The modelling process converted the $(\alpha, \delta, V)$ channel maps to a set of velocity profiles $\left(\alpha, \delta, \Psi_{\mathfrak{i}}\right)$. All subsequent analysis used the modelled velocity profiles as functions of position.

\subsection{Analysis}

\subsubsection{Clump Identification}

Both the channel maps and the modelled profiles show opacity variations $\Delta \tau / \tau$ of $50 \%$ or more on scales down to the beam size. We wish to isolate identifiable clumps to use the opacity profiles at clump center as an estimator of the relative level populations and hence the CMB temperature. We used the following algorithm to identify clump candidates. First, we generated a map of opacity vs position for all pixels with modelled velocity centroids in a given range defined by the velocity separation of the features. Toward W51d, cuts at $63-66.5$ and $67-69.5 \mathrm{~km} \mathrm{~s}^{-1}$ served to separate the two major spectral features. Toward W5le, where the morphology was more complicated, we used cuts at $61-63,63-67,66.5-68$, and $67.5-70 \mathrm{~km} \mathrm{~s}^{-1}$. We took as clump candidates those pixels with opacity enhancement $>50 \%$ over surrounding areas, and rejected any consisting of fewer than 8 contiguous pixels (half of the synthesized beam area). We then compared 

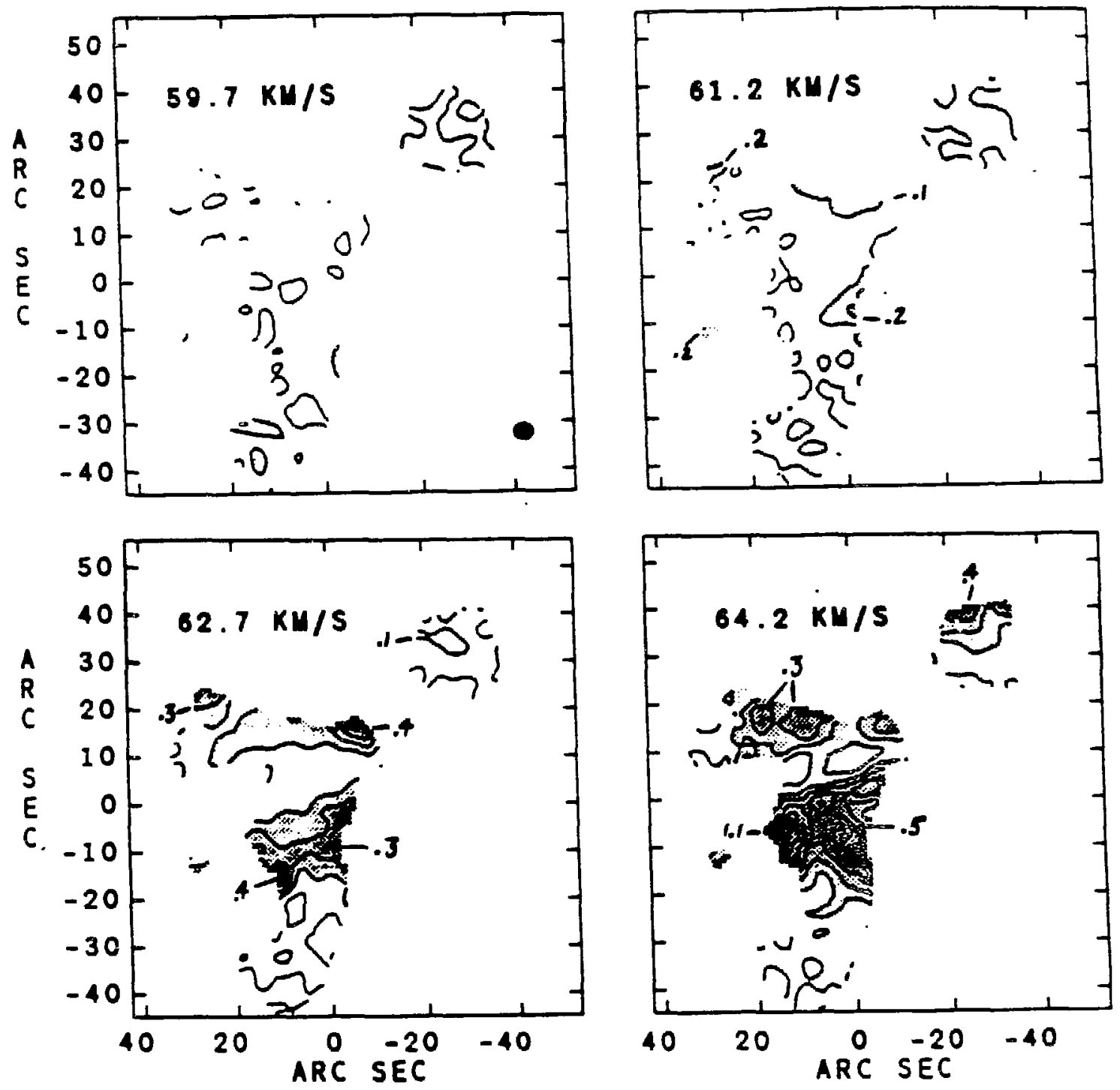

Figure 5.4: Contour plots of the opacity at $6 \mathrm{~cm}$. The LSR velocity of each channel map is indicated. The lowest contour is at 0.0 opacity; successive contours are spaced by 0.1 to a maximum of 1.2. The grey scale distinguishes regions of increasing and decreasing opacity. The beam size is indicated by the shaded oval at the bottom right comer of the first plot. The reference position is $\alpha=19^{\mathrm{h}} 21^{\mathrm{m}} 24^{\mathrm{s}}, \delta=14^{\circ} 24^{\prime} 45^{\prime \prime}$ (1950.0) 

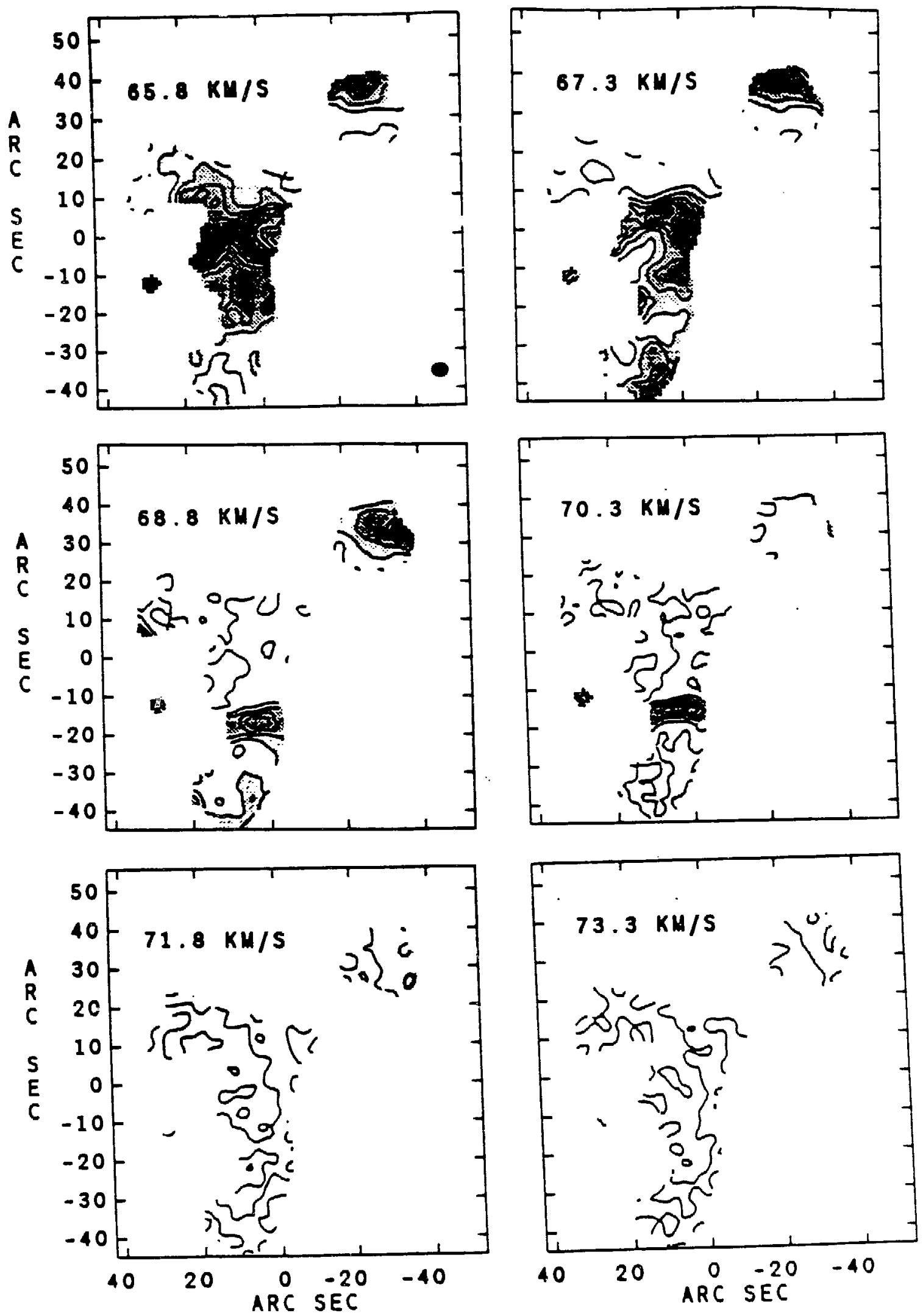

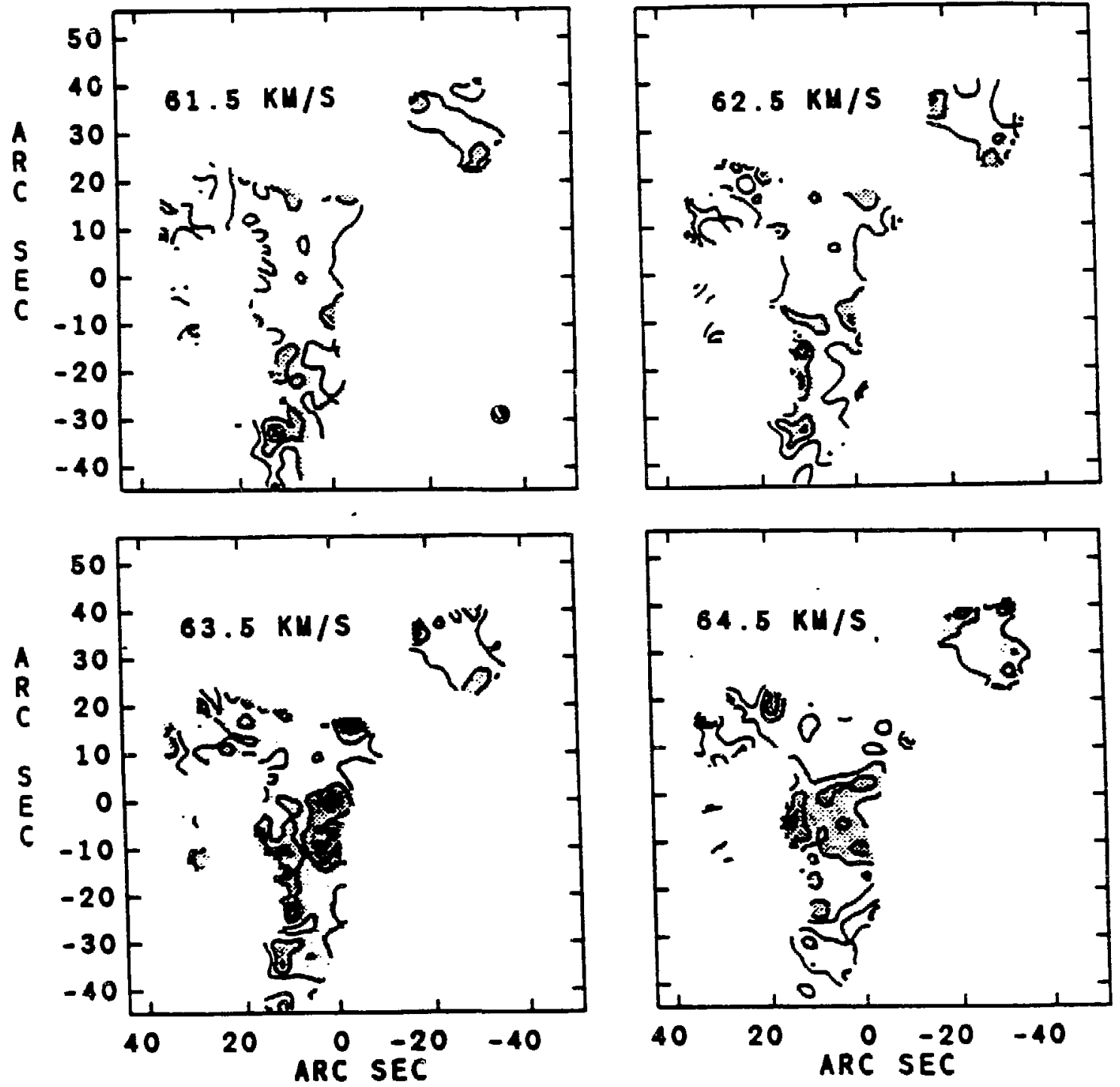

Figure 5.5: Plots of the opacity in the $2-\mathrm{cm}$ transition. The contours are as in Figure 5.4 . 

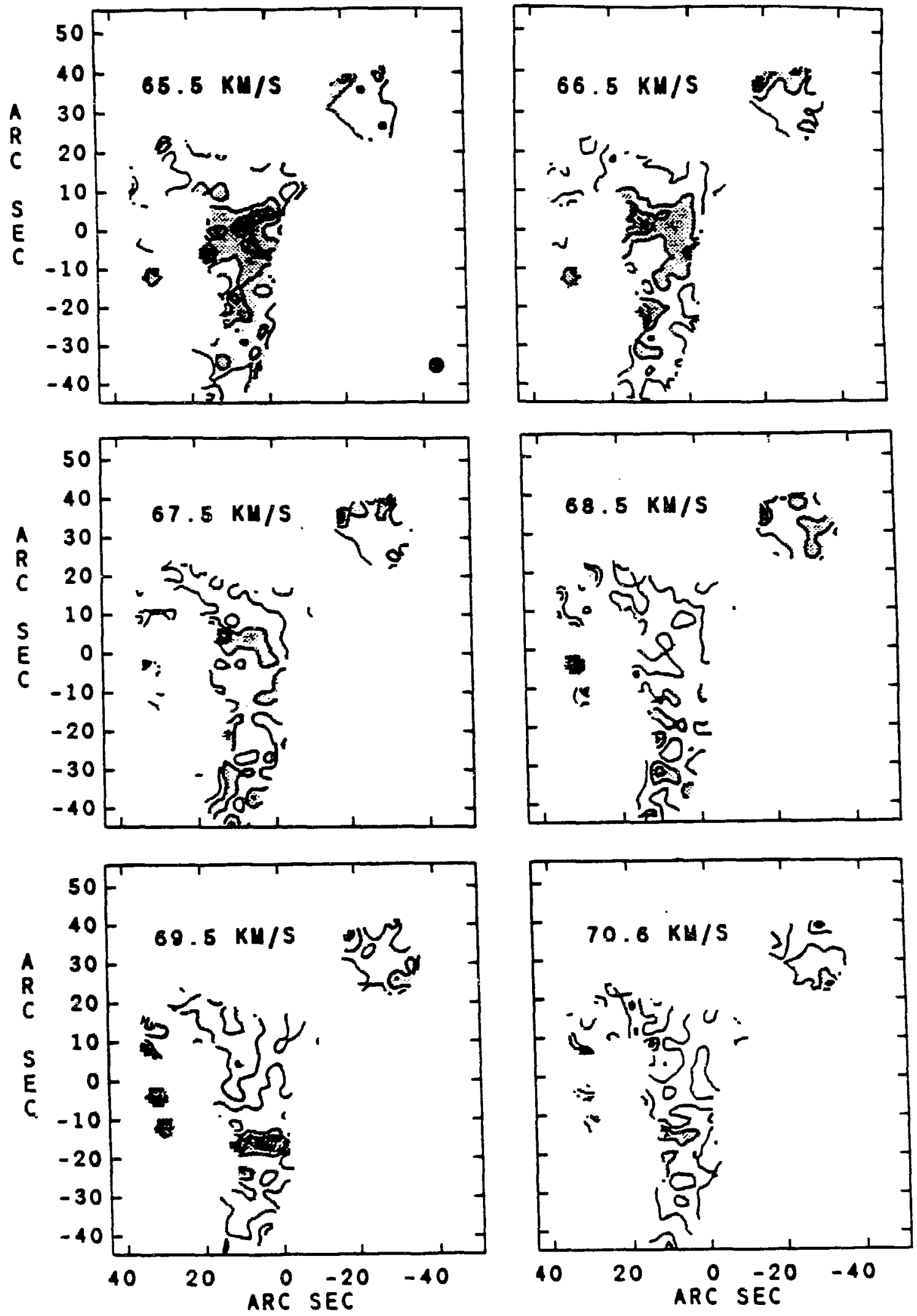


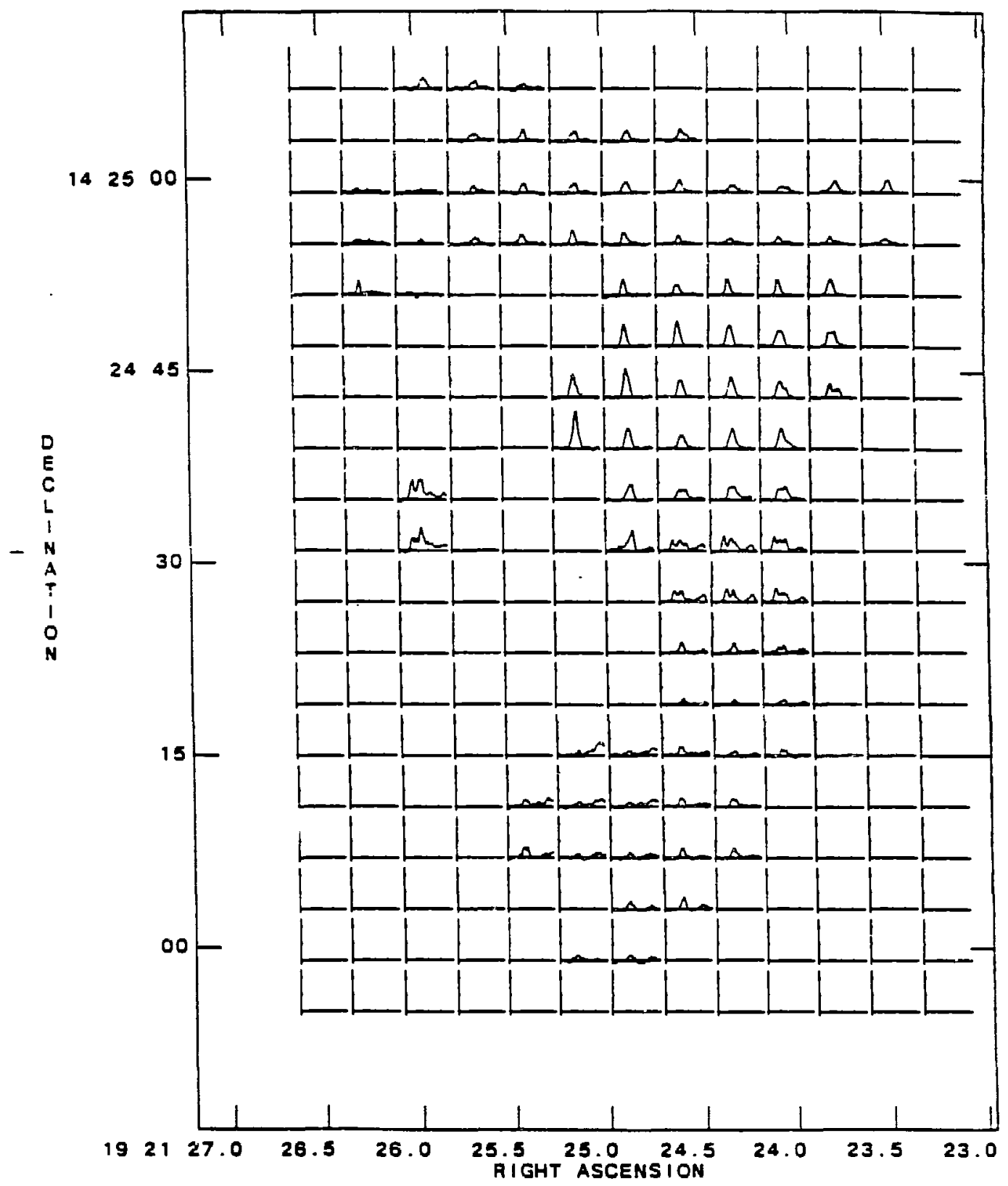

Figure 5.6: Spectra toward W5 1 e at $6 \mathrm{~cm}$ are displayed as a function of position across the brighter continum source. The $x$ axis (velocity) runs from 53 to $76 \mathrm{~km} \mathrm{~s}^{-1}$; the $y$ axis (opacity) from 0.0 to 0.1 . 
the cuts at $6 \mathrm{~cm}$ and $2 \mathrm{~cm}$, and rejected any candidates which did not appear at the same position $(\alpha, \delta)$ at both frequencies. This last test served to reject noisy data, but does introduce a bias against weak opacity features, which may not appear in the noisier 2-cm spectra. For example, although both the channel maps and the modelled profiles clearly show absorption at $61 \mathrm{~km} \mathrm{~s}^{-1}$, it is patchy and does not appear in quite the same positions at both frequencies. The clumps identified are not an exhaustive list, but represent a sample yielding to reac; parametrization.

Table 5.4 lists nine clumps identified using the algorithm above. The data are for the clump center, the spectral profile with the largest local opacity enhancement. The center velocity and FWHM are taken from the 6 -cm spectra, which had better signal to noise. The velocity profiies at $6 \mathrm{~cm}$ are in excellent agreement with the $2-\mathrm{cm}$ spectra. Error estimates are $68 \%$ confidence level statistical limits based on the assumption of gaussian noise in the outermost channels. The clumps are mainly in the velocity range $64-66 \mathrm{~km} \mathrm{~s}^{-1}$ associated with foreground molecular clouds; none are associated in position or velocity with the massive core regions.

\subsubsection{Clump Radius}

The estimate of the radius of each clump is model-dependent. For a sphere of constant density and radius $R$, the opacity in a line of sight displaced a distance $d$ from clump center will vary according to the length of the chord,

$$
\frac{\tau}{\tau_{0}}=\left[1-\left(\frac{d}{R}\right)^{2}\right]^{1 / 2}
$$

Convolved with the 4" FWHM of the beam, this fixes the ratio of opacity at clump center to opacity when the beam center is at the edge of the clump. The convolved opacity ratio $t / \tau_{0}$ is dependent on the ratio $\mathrm{R} / \mathrm{W}$ where $\mathrm{W}$ is the beam width; we calculated the radii iteratively to achieve a self-consistent result. The clumps suffer from some superposition; we therefore calculated $\mathrm{R}$ (using Eq. 5.13) for the largest opacity clump, subtracted the resultant opacity gradient from the maps, and proceeded to the densest remaining clump. By restricting analysis to regions with bright background continuum, we reduce effects of continuum uncertainty and morphology in the determination of clump radius. 
The velocity structure of the clouds can be used as a cross-check on the estimates of clump radii. From Eq. 5.9, the clumps should be marked by a linear velocity gradient $\Delta \mathrm{V} / \Delta \mathrm{L}$, with the velocity difference $\Delta \mathrm{V}$ equal to the line width $\sigma_{\mathrm{V}}$

$$
\mathrm{R} \frac{\mathrm{dv}}{\mathrm{dr}} \approx \Delta \mathrm{V} \approx \sigma_{\mathrm{v}}
$$

Figure 5.7 shows the velocity structure of the clouds toward W51 for strips of constant right ascension centered on several of the clumps. The arrows indicate the position of clump center inferred from the opacity structure. The clouds are marked by linear velocity gradients over extended regions. The line width $\sigma_{\mathrm{v}}$, total velocity difference $\Delta \mathrm{V}$ over transverse distance $\Delta \mathrm{L}$, and the velocity derivative $\mathrm{dv} / \mathrm{dr}$ are given in Table 5.5. The transverse extent $\Delta L$ of the linear velocity field, radius $R_{\tau}$ derived from the opacity, and the radius $R_{\mathbf{V}}$ defined by

$$
R_{v}=\frac{\sigma_{v}}{d v / d r}
$$

can be compared as a test of the assumption of spherical geometry. The ratio $R_{v} / R_{\tau}$ has a mean value $1.1 \pm 0.2$ for clumps $1-8$, while the ratio $\Delta L / R_{\tau}$ has a mean $1.1 \pm 0.1$. The data have scatter of $-50 \%$. The clumps as a set agree with the LVG model at the $10 \%$ level. Only clump 9 is in serious disagreement with the assumption of a spherical geometry. The southernmost region toward W51e shows very little transverse velocity gradient yet has a substantial line width. Since this clump does not agree with the assumed geometry used in the radiative transfer model, it has been neglected in all further analysis.

\subsubsection{Radiative Transfer Model}

The line width $\sigma_{v}$, radius $R$, and opacities $\tau_{6 \mathrm{~cm}}$ and $\tau_{2 \mathrm{~cm}}$ form a set of observables for each clump, from which we wish to determine the $\mathrm{CMB}$ temperature $\mathrm{T}_{\mathrm{CMB}}$. In the absence of collisional pumping, the opacity ratio $\tau_{6 \mathrm{~cm}} / \tau_{2 \mathrm{~cm}}$ is a single-valued function of $\mathrm{T}_{\mathrm{CMB}}$, allowing Eqs. 5.3, 5.6, 5.10, and 5.11 to be solved for the temperature of the radiation field (assumed to be dominated by a blackbody) within each clump. In the presence of collisional pumping, the opacity ratio becomes a function of CMB temperature, hydrogen density, and kinetic temperature. The hydrogen space density $n\left(\mathrm{H}_{2}\right)$ and kinetic temperature $T_{\text {kin }}$ are not directly observable in the $\mathrm{H}_{2} \mathrm{CO}$ transitions, but must be modelled using information from other sources. 


\begin{tabular}{ccccccc}
\hline Clump & $\alpha$ & $\delta$ & $\mathrm{V}$ & \multicolumn{2}{c}{ FWHM } & \multicolumn{2}{c}{ Optical Depth } \\
& $(1950.0)$ & $(1950.0)$ & $\left(\mathrm{km} \mathrm{s}^{-1}\right)$ & $\left(\mathrm{km} \mathrm{s}^{-1}\right)$ & $6 \mathrm{~cm}$ & $2 \mathrm{~cm}$ \\
\hline 1 & $19^{\mathrm{h}} 21^{\mathrm{m}} 24 . \mathrm{s} 93$ & $14^{\circ} 24^{\prime} 39^{\prime \prime}$ & 65.1 & $2.66 \pm 0.05$ & $1.004 \pm 0.014$ & $0.335 \pm 0.017$ \\
2 & $19^{\mathrm{h}} 21^{\mathrm{m}} 24 .^{\mathrm{s}} 20$ & $14^{\circ} 24^{\prime} 38^{\prime \prime}$ & 65.1 & $3.53 \pm 0.05$ & $0.585 \pm 0.007$ & $0.192 \pm 0.008$ \\
3 & $19^{\mathrm{h}} 21^{\mathrm{m}} 23 . \mathrm{s} 73$ & $14^{\circ} 24^{\prime} 49^{\prime \prime}$ & 65.7 & $3.62 \pm 0.07$ & $0.509 \pm 0.007$ & $0.228 \pm 0.018$ \\
4 & $19^{\mathrm{h}} 21^{\mathrm{m}} 24 . \mathrm{s} 67$ & $14^{\circ} 24^{\prime} 58^{\prime \prime}$ & 64.7 & $3.29 \pm 0.09$ & $0.374 \pm 0.008$ & $0.103 \pm 0.011$ \\
5 & $19^{\mathrm{h}} 21^{\mathrm{m}} 25 . \mathrm{s} 27$ & $14^{\circ} 25^{\prime} 01^{\prime \prime}$ & 64.3 & $2.49 \pm 0.19$ & $0.343 \pm 0.022$ & $0.160 \pm 0.025$ \\
6 & $19^{\mathrm{h}} 21^{\mathrm{m}} 22 . \mathrm{s} 20$ & $14^{\circ} 25^{\prime} 24^{\prime \prime}$ & 65.7 & $3.71 \pm 0.21$ & $0.501 \pm 0.023$ & $0.173 \pm 0.045$ \\
7 & $19^{\mathrm{h}} 21^{\mathrm{m}} 21 . \mathrm{s} 53$ & $14^{\circ} 25^{\prime} 17^{\prime \prime}$ & 68.7 & $1.88 \pm 0.21$ & $0.536 \pm 0.031$ & $0.290 \pm 0.045$ \\
8 & $19^{\mathrm{h}} 21^{\mathrm{m}} 24 . \mathrm{s}^{\prime} 07$ & $1^{\circ} 24^{\prime} 28^{\prime \prime}$ & 69.6 & $1.62 \pm 0.09$ & $0.714 \pm 0.032$ & $0.379 \pm 0.030$ \\
9 & $19^{\mathrm{h}} 21^{\mathrm{m}} 24 . \mathrm{s}^{\prime} 40$ & $14^{\circ} 24^{\prime} 07^{\prime \prime}$ & 67.4 & $2.47 \pm 0.16$ & $0.364 \pm 0.019$ & $0.129 \pm 0.028$ \\
\hline
\end{tabular}

Table 5.4: Summary of clump properties. Estimated uncertainties are statistical only.

\begin{tabular}{ccccccc}
\hline \hline Clump & $\begin{array}{c}\sigma_{v} \\
\left(\mathrm{~km} \mathrm{~s}^{-1}\right)\end{array}$ & $\begin{array}{c}\Delta V \\
\left(\mathrm{~km} \mathrm{~s}^{-1}\right)\end{array}$ & $\begin{array}{c}\frac{\mathrm{dv}}{\mathrm{dr}} \\
\left(\mathrm{km} \mathrm{s}^{-1} \mathrm{pc}^{-1}\right)\end{array}$ & $\begin{array}{c}\Delta \mathrm{L} \\
(\mathrm{pc})\end{array}$ & $\begin{array}{c}\mathbf{R}_{\mathrm{v}} \\
(\mathbf{p c})\end{array}$ & $\begin{array}{c}\mathbf{R}_{\tau} \\
(\mathrm{pc})\end{array}$ \\
\hline 1 & $1.13 \pm 0.02$ & $2.14 \pm 0.07$ & $4.9 \pm 0.3$ & $0.44 \pm 0.04$ & $0.23 \pm 0.01$ & $0.50 \pm 0.11$ \\
2 & $1.50 \pm 0.02$ & $1.78 \pm 0.03$ & $7.1 \pm 0.3$ & $0.25 \pm 0.04$ & $0.21 \pm 0.01$ & $0.20 \pm 0.03$ \\
3 & $1.54 \pm 0.03$ & $0.57 \pm 0.06$ & $3.8 \pm 0.5$ & $0.15 \pm 0.04$ & $0.40 \pm 0.06$ & $0.21 \pm 0.03$ \\
4 & $1.40 \pm 0.04$ & $1.26 \pm 0.06$ & $4.1 \pm 0.3$ & $0.29 \pm 0.04$ & $0.34 \pm 0.03$ & $0.16 \pm 0.01$ \\
5 & $1.06 \pm 0.08$ & $0.89 \pm 0.14$ & $6.0 \pm 0.8$ & $0.15 \pm 0.04$ & $0.17 \pm 0.02$ & $0.17 \pm 0.01$ \\
6 & $1.58 \pm 0.09$ & $1.53 \pm 0.11$ & $5.5 \pm 0.3$ & $0.36 \pm 0.04$ & $0.29 \pm 0.02$ & $0.28 \pm 0.03$ \\
7 & $0.80 \pm 0.09$ & $0.94 \pm 0.15$ & $4.4 \pm 0.8$ & $0.18 \pm 0.04$ & $0.18 \pm 0.04$ & $0.22 \pm 0.09$ \\
8 & $0.69 \pm 0.04$ & $1.86 \pm 0.16$ & $5.2 \pm 0.5$ & $0.40 \pm 0.04$ & $0.13 \pm 0.01$ & $0.27 \pm 0.25$ \\
9 & $1.05 \pm 0.07$ & $0.25 \pm 0.08$ & $0.8 \pm 0.3$ & $0.25 \pm 0.04$ & $1.3 \pm 0.4$ & $0.21 \pm 0.02$ \\
\hline \hline
\end{tabular}

Table 5.5: Clump velocity gradients and radii. Estimated uncertainties are statistical only. 

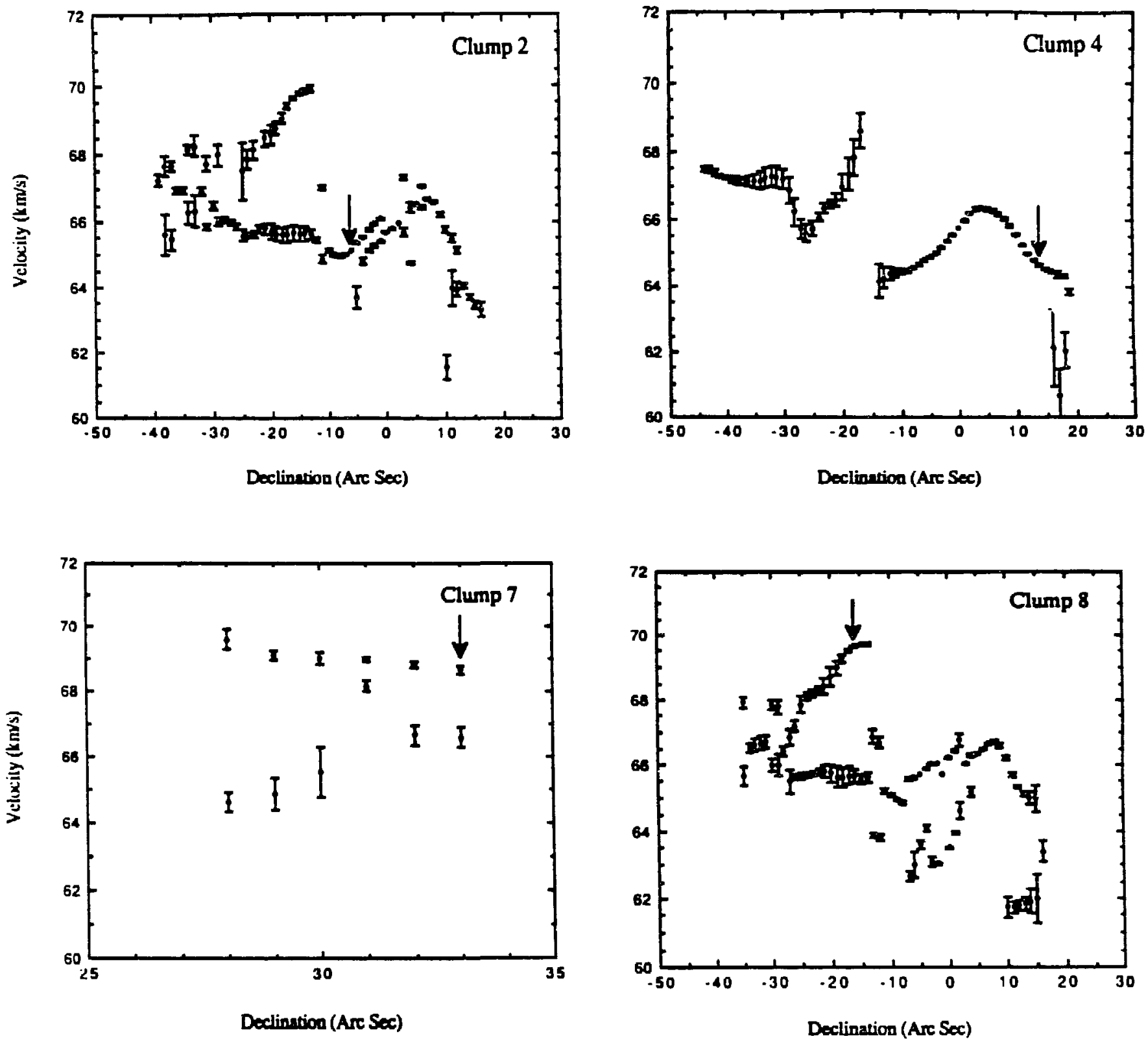

Figure 5.7: Velocity centroids of the fitted 6-cm spectra plotted vs declination for selected strips centered on the clumps. The arrows note the location of the clump center inferred from the opacity maps. 
The kinetic temperature of the foreground clouds may be deduced from observations of ${ }^{12} \mathrm{CO}$ emission toward W51. The $\mathrm{J}=1-0$ transition of ${ }^{12} \mathrm{CO}$ at $2.6 \mathrm{~mm}$ is optically thick and thermalizes to the kinetic temperature of the molecular clouds. Observations of ${ }^{12} \mathrm{CO}$ at 70 angular resolution indicate a kinetic temperature in the $64-67 \mathrm{~km} \mathrm{~s}-1$ features of $11 \pm 2$ $\mathrm{K}$ (Wilson et al. 1974, Plambeck et al. 1983). This value is an average over a much broader beam than our 4 " resolution. The kinetic temperature could conceivably be higher in the small, denser regions characterized by the clumps. As $\partial \mathrm{T}_{\mathrm{CMB}} / \partial \eta_{\mathrm{kin}}>0$, uncertainties in $T_{k i n}$ from beam-filling factors will serve to raise the estimate of $T_{C M B}$. The temperature rises to $50 \mathrm{~K}$ in the molecular clouds associated with the hot, dense core; however, none of the clumps used are associated with the core. The observed temperature of the foreground at locations $3 \mathrm{pc}$ apart agrees within $0.5 \mathrm{~K}$; consequently, we take the kinetic temperature of the foreground clouds containing the clumps to be $11 \pm 2 \mathrm{~K}$.

The space density of molecular hydrogen may be estimated from the column density of $\mathrm{H}_{2} \mathrm{CO}$, the radius of the clump, and the abundance ratio $\mathrm{X}$ between $\mathrm{H}_{2} \mathrm{CO}$ and $\mathrm{H}_{2}$,

$$
X=\frac{n\left(\mathrm{H}_{2} \mathrm{CO}\right)}{\mathrm{n}\left(\mathrm{H}_{2}\right)}
$$

The abundance ratio has been determined for a number of molecular clouds (Wadiak et al. 1988, Mundy et al. 1987, and references therein). The data show a general trend toward lower $\mathrm{X}$ at higher $\mathrm{H}_{2}$ densities, perhaps caused by depletion of $\mathrm{H}_{2} \mathrm{CO}$ onto grains. A power-law variation of $\mathrm{X}$ with $\mathrm{H}_{2}$ density yields the relation

$$
X=[2.0 \pm 0.5] \times 10^{-9}\left[\frac{\mathrm{n}\left(\mathrm{H}_{2}\right)}{8 \times 10^{4} \mathrm{~cm}^{-3}}\right]^{-0.5 \pm 0.1}
$$

which we adopt for the clouds toward W51.

The collisional excitation rates $\gamma_{\mathrm{ij}}$ have been calculated for the $\mathrm{He}-\mathrm{H}_{2} \mathrm{CO}$ system by Green et al. (1978). The authors argue that the individual rates for the $\mathrm{H}_{2}-\mathrm{H}_{2} \mathrm{CO}$ system may differ by $20 \%$, but should have similar overall behavior. The greater polarizability of $\mathrm{H}_{2}$ increases the rates by $20 \%$ above the $\mathrm{He}-\mathrm{H}_{2} \mathrm{CO}$ rates, and the smaller reduced mass of $\mathrm{H}_{2}$ increases the rates by an additional $30 \%$. Our model used the $\mathrm{He}-\mathrm{H}_{2} \mathrm{CO}$ rates of Green et al. multiplied by a constant factor of $1.5 \pm 0.2$.

We now have enough information to solve Eqs. 5.3-5.11 in the context of a spherically symmetric LVG model. The observables $\left(\sigma_{v}, R_{\tau}, \tau_{6 \mathrm{~cm}}, \tau_{2 \mathrm{~cm}}\right)$ for each clump yield the $\mathrm{CMB}$ temperature and $\mathrm{H}_{2}$ density from the following algorithm. 
1) The kinetic temperature $T_{k i n}$ is set to the nominal value $T_{\text {kin }}=11 \mathrm{~K}$.

2) The $\mathrm{CMB}$ temperature is set to an initial value of $\mathrm{T}_{\mathrm{CMB}}=0.1 \mathrm{~K}$

3) The hydrogen density is set to a start value of $n\left(\mathrm{H}_{2}\right)=10^{6} \mathrm{~cm}^{-3}$.

4) The $\mathrm{H}_{2} \mathrm{CO}$ density is calculated from the current value for $n\left(\mathrm{H}_{2}\right)$ and the abundance ratio $X$ (Eq. 5.15).

5) Equations 5.3-5.6 form a set of linear equations, which we solve for the level populations $n_{i}$ in the first 14 rotational transitions of orthoformaldehyde. The level populations determine the opacities $\tau_{\mathrm{ij}}$ and escape probabilities $\beta_{\mathrm{ij}}$ for each of the transitions (Eq. 5.10, 5.11). Step 5 repeats until the escape probabilities are stable with respect to further iterations.

6) The hydrogen density $n\left(\mathrm{H}_{2}\right)$ is varied (with $T_{C M B}$ constant) and steps 4-6 repeated until the calculated opacity ratio $\tau_{6 \mathrm{~cm}} / \tau_{2 \mathrm{~cm}}$ matches the observed ratio within pre-determined limits.

7) The CMB temperature is varied, and the algorithm repeats steps 3-7 until both $\tau_{6 \mathrm{~cm}}$ and the ratio $\tau_{6 \mathrm{~cm}} / \tau_{2 \mathrm{~cm}}$ match the observations within pre-determined limits.

We evaluated the uncertainties numerically, by varying independently each of the observables over the range defined by the $68 \% \mathrm{CL}$ limits, with all other parameters held constant at their mean values. A similar treatment for the systematic parameters $T_{\text {kin }}, X$, and $\gamma_{i j}$ yielded estimates of the systematic uncertainties associated with each parameter.

\subsection{Results}

\subsubsection{Hydrogen Density}

The radiative transfer model provides an estimate of the local hydrogen space density within each clump. Values for each of the clumps are listed in Table 5.6. Although $\mathrm{n}\left(\mathrm{H}_{2}\right)$, unlike $\mathrm{T}_{\mathrm{CMB}}$, is not a universal constant, the mean value is useful as an estimate of the 


\begin{tabular}{lccc}
\hline Clump & $\begin{array}{c}\mathrm{n}\left(\mathrm{H}_{2}\right) \\
\left(10^{4} \mathrm{~cm}^{-3}\right)\end{array}$ & $\begin{array}{c}\mathrm{M} \\
(\mathrm{M} \theta)\end{array}$ & $\begin{array}{c}\Delta \mathrm{V} \\
\left(\mathrm{km} \mathrm{s}^{-1}\right)\end{array}$ \\
\hline 1 & $1.1 \pm 0.2$ & 330 & 1.1 \\
2 & $1.6 \pm 0.3$ & 140 & 0.9 \\
3 & $3.6 \pm 0.7$ & 80 & 0.8 \\
4 & $2.0 \pm 0.4$ & 20 & 0.5 \\
5 & $2.9 \pm 0.6$ & 30 & 0.6 \\
6 & $2.0 \pm 0.4$ & 105 & 0.8 \\
7 & $2.5 \pm 0.5$ & 65 & 0.7 \\
8 & $2.1 \pm 0.4$ & 100 & 0.8 \\
\hline
\end{tabular}

Table 5.6: Derived $\mathrm{H}_{2}$ space density, mass, and velocity associated with each clump.

\begin{tabular}{cc}
\hline Clump & $\mathrm{T}_{\text {CMB }}(\mathrm{K})$ \\
\hline 1 & $2.44 \pm 1.67$ \\
2 & $3.04 \pm 0.73$ \\
3 & $0.00 \pm 1.69$ \\
4 & $2.85 \pm 0.31$ \\
5 & $5.26 \pm 0.59$ \\
6 & $3.19 \pm 0.73$ \\
7 & $5.01 \pm 3.19$ \\
8 & $4.57 \pm 4.59$ \\
\hline Weighted Average & $3.3 \pm 0.2$ \\
\hline
\end{tabular}

Table 5.7: $\mathrm{CMB}$ thermodynamic temperature derived from $\mathrm{H}_{2} \mathrm{CO}$ for each slump. The error estimates are based on variations of the input opacities, line widths, and radii of the clumps within their $68 \%$ CL limits. 
densities typical within the c.umps. The eight clumps modelled have a mean value for $\mathrm{n}\left(\mathrm{H}_{2}\right)$ of

$$
\mathrm{n}\left(\mathrm{H}_{2}\right)=(2.0 \pm 0.9) \times 10^{4} \mathrm{~cm}^{-3}
$$

This value is midway between the values determined by Amal et al (1985) and MartinPintado et al. (1985). As it depends on the assumed abundance ratio X (Eq. 5.15), it does not constitute an independent test of the variation of $\mathrm{n}\left(\mathrm{H}_{2} \mathrm{CO}\right)$ with $\mathrm{n}\left(\mathrm{H}_{2}\right)$.

The derived $\mathrm{H}_{2}$ density can be used as a cross-check on the LVG model. From Eq. 5.9 , a clump of radius $R$ and mass density $p$ should have an associated velocity

$$
\begin{aligned}
\Delta V & =[2 \mathrm{G} \rho]^{0.5} \mathrm{R} \\
& =2.1\left(\frac{\mathrm{n}\left(\mathrm{H}_{2}\right)}{10^{4} \mathrm{~cm}^{-3}}\right)^{0.5}\left(\frac{\mathrm{R}}{1 \mathrm{pc}}\right) \mathrm{km} \mathrm{s}^{-1}
\end{aligned}
$$

Table 5.6 lists the $\mathrm{H}_{2}$ space density, total mass, and velocity derived for each clump. The derived velocity gradients for the clumps are higher than the observed values by a factor of 1.8 , with a clump-to-clump scatter of $60 \%$.

\subsubsection{Mean CMB Temperature}

The CMB temperature determined from each clump is listed in Table 5.7. The quoted uncertainty for each clump is the quadrature sum of the uncertainties in $\mathrm{T}_{\mathrm{CMB}}$ induced by the statistical uncertainties of the input parameters $\sigma_{v}, R, \tau_{6 \mathrm{~cm}}$, and $\tau_{2 \mathrm{~cm}}$. The largest contribution to the statistical uncertainty is the uncertainty in radius of each clump, followed by the opacity in the $2 \mathrm{~cm}$ transition.

Errors in $\mathrm{T}_{\mathrm{CMB}}$ arising from statistical uncertainties in the input clump parameters are not expected to correlate; consequently, we derive a single CMB temperature from the eight clumps by taking an average of the clumps weighted by their statistical uncertainties. The resultant value is

$$
\mathrm{T}_{\mathrm{CMB}}=3.3 \pm 0.2 \mathrm{~K} \text { (statistical uncertainty only). }
$$




\subsubsection{Systematic Uncertainty}

Uncertainties in $\mathrm{T}_{\mathrm{CMB}}$ arising from propagated errors in the radiative transfer model are correlated from clump to clump and have not been included in the weighted average above. We estimated their magnitude by running the computer code with values for $T_{k i n}$, $\mathrm{X}$, and the collisional rates $\gamma_{\mathrm{ij}}$ altered from their nominal values. For each run, we determined the weighted average $\mathrm{T}_{\mathrm{CMB}}$ as in the previous section. We took the difference between the nominal $\mathrm{T}_{\mathrm{CMB}}$ and the new value as the magnitude of the uncertainty associated with the parameter being varied.

We have modelled the radiation field in the molecular clouds as dominated by a blackbody at temperature $\mathrm{T}_{\mathrm{CMB}}$. Since large molecular clouds are frequently associated with star-forming regions, the possibility exists for significant contributions to the radiation field from the IR emission of young stars or greybody emission from the $-11 \mathrm{~K}$ dust. The clouds toward W51 have been studied at a number of frequencies, allowing us to resolve this problem.

Maps at 50,100, and 500 microns show the IR continuum to be centered on the compact $\mathrm{H} n$ regions W5 $\mathrm{Ce}_{1}$ and W5le 2 and to a lesser extent on the region IRS2 toward the region W51d (Harvey et al. 1986, Jaffe et al. 1984). Most of the mass is in the massive core toward W5 $\mathrm{le}_{1}$ and W5le2. There is no indication of hot regions toward the clumps studied in this work, which are in the foreground. Studies of molecular emission reinforce this point. $\mathrm{NH}_{3}$ emission and maser activity are frequently associated with starforming regions. VLA maps of $\mathrm{NH}_{3}$ emission toward $\mathrm{W} 51$ at the $\mathrm{LSR}$ velocities of the clumps show the emission to be centered on the regions of $\mathrm{OH}$ and $\mathrm{H}_{2} \mathrm{O}$ maser activity toward W51e 1, e2, and IRS2 (Ho et al. 1983). No emission is detected toward the cold foreground clouds containing the clumps. We conclude that there is no IR source in these clouds.

Greybody emission from dust in the clouds is another possible contaminant of the local $\mathrm{mm}$ field. We have included greybody emission from dust in the radiation field used by our model. We adopted the dust opacity at $100 \mu, \tau_{100}=0.3$, taken from observations of the warmer clouds toward W51 at $57 \mathrm{~km} \mathrm{~s}^{-1}$ (Harvey et al. 1986) as a value typical for the colder dust at $66 \mathrm{~km} \mathrm{~s}^{-1}$. With emissivity scaled as $\lambda^{-2}$ (Draine et al. 1984), the dust changed the estimate of $\mathrm{T}_{\mathrm{CMB}}$ by less than $0.05 \mathrm{~K}$. This limit is small compared to other systematic sources of uncertainty. 
The estimated systematic uncertainties are listed in Table 5.8. Uncertainties in the kinetic temperature, abundance ratio, collisional rates, and dust emission are not correlated with each other, although they are invariant from clump to clump. We took the quadrature sum of the systematic uncertainty from each term as the estimate of the total systematic uncertainty of the measurement. The resultant value, $0.9 \mathrm{~K}$, was added in quadrature with the statistical uncertainty of the weighted average from the eight clumps to yield the final result

$$
\mathrm{T}_{\mathrm{CMB}}=3.2 \pm 0.9 \mathrm{~K}(2.1 \mathrm{~mm})
$$

\subsection{Discussion}

The CMB temperature at $2.1 \mathrm{~mm}$ is in agreement with a $2.7 \mathrm{~K}$ blackbody, although the large uncertainty makes the $\mathrm{H}_{2} \mathrm{CO}$ measurement of limited use with respect to the $\mathrm{CMB}$ spectrum. It is not consistent with a zero temperature, however, and provides additional confirmation of the presence of the CMB throughout our Galaxy. In this respect, the measurement provides evidence for the growing utility of interstellar spectroscopy as a remote probe of the $\mathrm{CMB}$.

The uncertainty in $\mathrm{T}_{\mathrm{CMB}}$ is dominated by systematic effects. As observations provide better estimates of the kinetic temperature and abundance ratic toward W51, the errors can be expected to decrease. Although it is highly unlikely that $\mathrm{H}_{2} \mathrm{CO}$ will ever approach the sensitivity of $\mathrm{CN}$ measurements, $\mathrm{H}_{2} \mathrm{CO}$ probes a much larger distance scale than $\mathrm{CN}$, even within the Galaxy.

More significantly, we have demonstrated that $\mathrm{H}_{2} \mathrm{CO}$ can be used to provide a $\mathrm{CMB}$ measurement in the presence of a large collisional pumping effect. The implications for a $\mathrm{CMB}$ detection at moderate redshift $(\mathrm{z}-1)$ are promising. $\mathrm{H}_{2} \mathrm{CO}$ is a ubiquiteus component of molecular clouds. It has the advantage over $\mathrm{CN}$ that the observed transitions are in the microwave, and the required continuum sources are HII regions instead of individual stars. $\mathrm{CN}$ is unlikely to provide a CMB detection outside the Galaxy, while $\mathrm{H}_{2} \mathrm{CO}$ has been observed in external galaxies in absorption against both continuum sources and the CMB(Graham et al. 1978, Cohen et al. 1979). The transition at $2.1 \mathrm{~mm}$ is near the CMB peak even at $z=1$; consequently, we can still expect significant population of the lower rotational levels. In this respect $\mathrm{H}_{2} \mathrm{CO}$ is superior to atomic measurements, which are in the Wien region of the $\mathrm{CMB}$ even at $z=2$. 
The uncertainty of the measurement is dominated by systematic effects associated with the molecular physics of the $\mathrm{H}_{2} \mathrm{CO}-\mathrm{H}_{2}$ system. As such, these uncertainties will not grow with redshift as rapidly as the $(1+z)$ dependence of the CMB signal. The signal to noise ratio would become the limiting factor for extragalactic observations. A positive CMB detection at significant redshift, even with $30 \%$ uncertainty, would be of great interest. We are currently investigating the feasibility of a search for $\mathrm{H}_{2} \mathrm{CO}$ absorption toward distant galaxies or QSOs.

\begin{tabular}{lcc}
\hline \hline \multicolumn{1}{c}{ Effect } & Magnitude & Uncertainty in TCMB (K) \\
\hline Collision Rates & $\pm 20 \%$ & \pm 0.15 \\
Kinetic Temperature & $\pm 2 \mathrm{~K}$ & \pm 0.45 \\
Abundance Ratio Magnitude & $(2.0 \pm 0.5) \times 10^{-9}$ & \pm 0.6 \\
Abundance Ratio Power Law & $0.5 \pm 0.1$ & \pm 0.45 \\
Dust Emission & Greybody at Tkin & $<0.05 \mathrm{~K}$ \\
\hline Total Estimated Systematics &.-- & $0.9 \mathrm{~K}$ \\
\hline \hline
\end{tabular}

Table 5.8: Systematic contributions to the error budget. 


\section{Chapter 6 \\ Analysis and Interpretation}

\subsection{Precise CMB Measurements}

Since 1980, a number of researchers have provided CMB measurements with greatly improved precision and spectral range. These data are summarized in Table 6.1 and plotted in Figure 6.1. Several general observations can be made about this data set. First, it should be noted that the uncertainties in these measurements are dominated by systematic effects. For measurements using similar techniques (e.g., the low-frequency measurements from Barcroft $[\lambda=20.5,7.9,4.0,3.0,0.91$, and $0.33 \mathrm{~cm}]$ or the $\mathrm{CN}$ measurements $[\lambda=0.264$ and $0.132 \mathrm{~cm}]$ ), some portion of the systematic uncertainty is correlated. The effect of correlated systematics is difficult to assess. Throughout this chapter, I shall assume that the correlation has negligible effect, and treat the entire uncertainty of each measurement as independent. At the present time, the data set is large enough that various subsets may be examined to obtain crude estimates of systematics. Assuming the means are gaussianly distributed within the uncertainties (and that no spectral distortions are present), the data weighted by $\sigma^{-2}$ will be a valid estimator of the mean of the parent distribution. Table 6.2 lists weighted means of various subsets of the data.

A natural division of the data is by spectral regime. The maximum brightness $B_{v}(T)$ of a blackbody at temperature $\mathrm{T}$ occurs at a frequency $\mathrm{v}_{0}$ given by the Wien displacement law,

$$
v_{0}=\frac{3 \mathrm{kT}}{\mathrm{h}}=60 \mathrm{~T} \mathrm{GHz}
$$

in terms of unit frequency, or

$$
\lambda_{0}=0.29 \mathrm{~T}^{-1} \mathrm{~cm}
$$

in terms of unit wavelength. For a $2.75 \mathrm{~K}$ blackbody, these are $165 \mathrm{GHz}$ and $0.11 \mathrm{~cm}$, respectively. I have separated the data set into a Rayleigh-Jeans set $\left(\lambda \triangleq \lambda_{0}\right)$, a transition region $\left(\lambda \sim \lambda_{0}\right)$, and a Wien set $\left(\lambda \sim \lambda_{0}\right)$, indicated by the thick lines in Table 6.1

Subsets of the full data set, separated by spectral regime, generally agree within $2 \sigma$. The high $\chi^{2}$ within each subset suggests that either the data do not follow gaussian statistics, that the errors are underestimated, or that CMB spectral features may be present. 


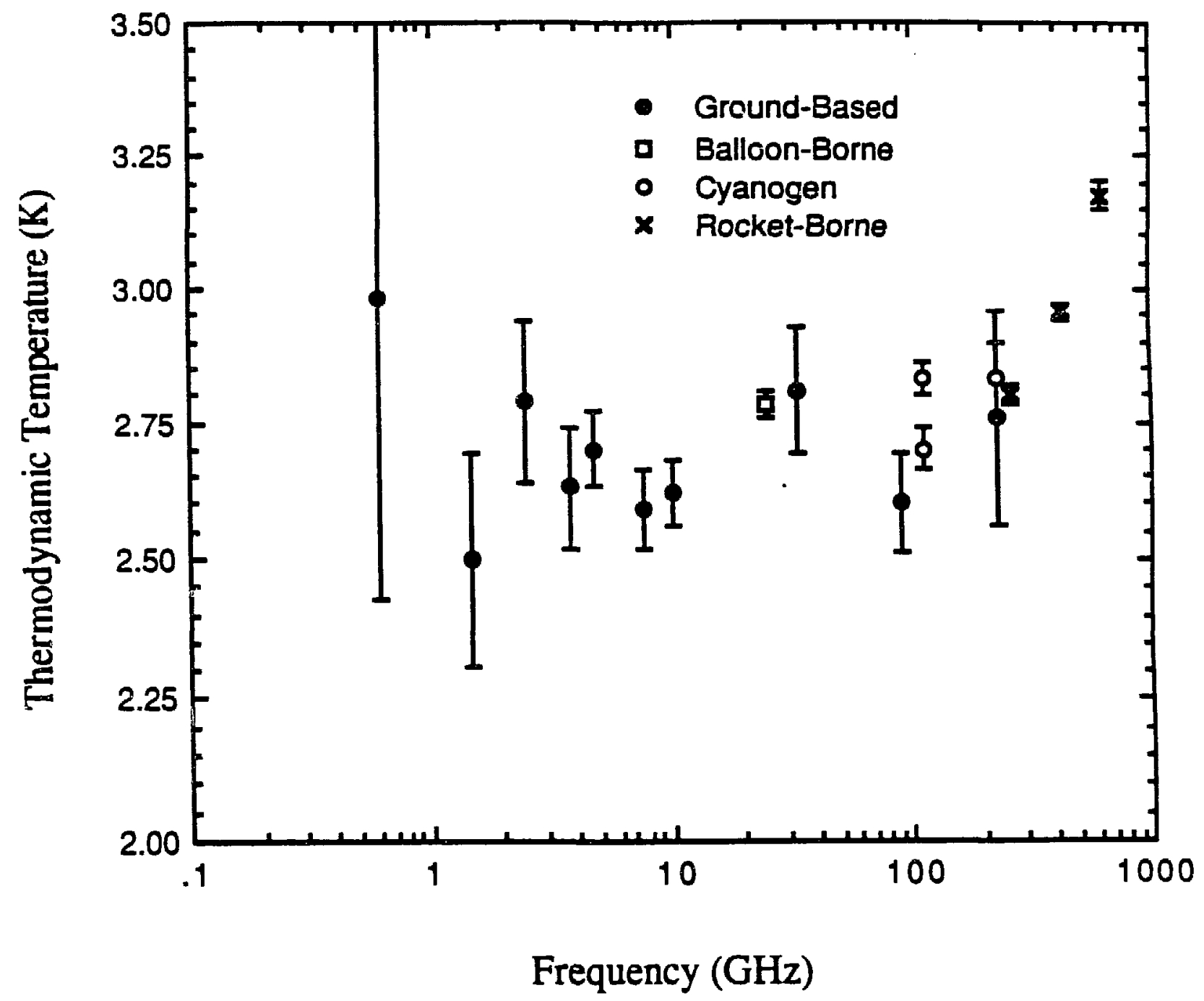

Figure 6.1: Recent measurements of the CMB spectrum. 


\begin{tabular}{|c|c|c|c|c|}
\hline Reference & $\begin{array}{l}\text { Wavelength } \\
\text { (cm) }\end{array}$ & $\begin{array}{c}\text { Frequency } \\
(\mathrm{GHz})\end{array}$ & $\begin{array}{c}\text { TCMB } \\
(\mathrm{K})\end{array}$ & Technique \\
\hline Sironi et al. 1987 & 50.0 & 0.6 & $2.98 \pm 0.55$ & GB \\
\hline Bensadoun (Smoot et al. 1989) & 20.3 & 1.47 & $2.50 \pm 0.19$ & GB \\
\hline Sironi and Bonelli 1986 & 12.0 & 2.5 & $2.79 \pm 0.15$ & GB \\
\hline De Amici (Smoot et al. 1989) & 7.9 & 3.8 & $2.63 \pm 0.11$ & GB \\
\hline Mandolesi et al. 1986 & 6.3 & 4.75 & $2.70 \pm 0.07$ & GB \\
\hline Kogut 1989 & 4.0 & 7.5 & $2.59 \pm 0.07$ & GB \\
\hline Kogut 1989 & 3.0 & 10.0 & $2.62 \pm 0.06$ & GB \\
\hline Johnson and Wilkinson 1986 & 1.2 & 24.8 & $2.783 \pm 0.025$ & BB \\
\hline De Amici et al. 1985 & 0.909 & 33.0 & $2.81 \pm 0.12$ & GB \\
\hline Bersanelli et al. 1989 & 0.333 & 90.0 & $2.60 \pm 0.09$ & GB \\
\hline \multirow[t]{2}{*}{ Meyer and Jura 1985} & 0.264 & 113.6 & $2.70 \pm 0.04$ & $\mathrm{CN}$ \\
\hline & 0.132 & 227.3 & $2.76 \pm 0.20$ & $\mathrm{CN}$ \\
\hline \multirow[t]{2}{*}{ Crane et al. 1988} & 0.264 & 113.6 & $2.796+0.041$ & $\mathrm{CN}$ \\
\hline & 0.132 & 227.3 & $2.85 \pm 0.10$ & $\mathrm{CN}$ \\
\hline Meyer et al. 1989 & 0.132 & 227.3 & $2.83 \pm 0.07$ & $\mathrm{CN}$ \\
\hline Matsumoto et al. 1988 & 0.116 & 259 & $2.799 \pm 0.018$ & $\mathbf{R}$ \\
\hline \multirow[t]{2}{*}{ Matsumoto et al. 1988} & 0.0709 & 423 & $2.955 \pm 0.017$ & $\mathbf{R}$ \\
\hline & 0.0481 & 624 & $3.175 \pm 0.027$ & $\mathbf{R}$ \\
\hline
\end{tabular}

Table 6.1: Recent precise measurements of the CMB temperature. The thick lines separate the Rayleigh-Jeans, intermediate, and Wien regions. "GB" indicates a ground-based measurement, "BB" a balloon-borme measurement, "CN" a spectroscopic measurement using the CN molecule, and "R" a rocket-borne experiment. "Kogut 1989" is this thesis.

\begin{tabular}{lccc}
\hline \hline Subset & Weighted Mean & $\chi^{2}$ & DOF \\
\hline Rayleigh-Jeans $(\lambda \geq 0.75 \mathrm{~cm})$ & $2.736 \pm 0.020$ & 15.1 & 8 \\
Intermediate $\left(0.75 \mathrm{~cm} \leq \lambda \leq \lambda_{0}\right)$ & $2.783 \pm 0.015$ & 12.1 & 6 \\
$\lambda \leq \lambda_{0}$ & $2.766 \pm 0.012$ & 32.1 & 15 \\
\hline Ground-Based & $2.646 \pm 0.031$ & 5.45 & 8 \\
Not Ground-Based & $2.786 \pm 0.013$ & 7.7 & 6 \\
Three Most Precise & $2.794 \pm 0.014$ & 0.52 & 2 \\
\hline
\end{tabular}

Table 6.2: Weighted means of various subsets of the spectral data set. 
Classifying subsets by observing technique sheds further light: now the subsets disagree at the $4 \sigma$ level, and are generally consistent internally. Ground-based measurements are systematically lower than other techniques by $0.14 \mathrm{~K}$. Since the most precise ground-based measurements are at $\lambda \geq 3 \mathrm{~cm}$, this can indicate either the presence of undetected systematic error in the measurements or a possible spectral distortion at long wavelengths. To distinguish between these two alternatives requires somewhat more sophisticated analysis.

\subsection{Mechanisms for Spectral Distortions}

Energy releasing processes in the early universe can produce distortions from a Planckian CMB spectrum, provided that the release leaves the matter and radiation fields out of equilibrium and that a mechanism exists to transfer energy between the matter and radiation. The present-day size, shape, and spectrum of the distortion will depend on the epoch and nature of the energy release.

The CMB does not probe the entire thermal history of the universe. At sufficiently early times, radiative processes such as double Compton scattering $(\gamma+e \leftrightarrow \gamma+\gamma+e)$ and thermal bremsstrahlung can completely thermalize an arbitrarily large energy release. Energy releases at times earlier than a characteristic redshift $z_{D C}$ result in a nearly exponential increase in photon number density with time until a Planckian distribution is established at a higher temperature. Danese and De Zotti (1982) estimate the limit of observable distortions to be

$$
\mathrm{z}_{\mathrm{DC}}=2.2 \times 10^{6} \Omega_{\mathrm{b}}{ }^{-1 / 3} \mathrm{~h}^{-2 / 3},
$$

where $h$ is defined as the Hubble constant in units of $50 \mathrm{~km} \mathrm{~s}^{-1} \mathrm{Mpc}^{-1}$ and $\Omega_{\mathrm{b}}$ is the baryon density of the universe relative to the critical density. For nominal values $\Omega_{b}-0.1$ and $h=1$, double Compton scattering limits the history of the universe observable in the CMB spectrum to $z_{0 b s} \leq 5 \times 10^{6}$, or energy scales $k T_{0}\left(1+z_{o b s}\right) \sim 1 \mathrm{keV}$. Processes at higher energy scales leave observable distortions only through such long-lived phenomena as particle decay.

At more recent epochs, the dominant interactions between charged particles and the CMB are non-radiative Compton scattering, which changes the photon energy but preserves the photon number, and bremsstrahlung, which generates new photons at low energies. At $\mathrm{z}<\mathrm{zDC}$, these processes are unable to regenerate a Planckian spectrum. It is 
convenient to parametrize distortions from a blackbody spectrum by the Planckian brightness temperature $T_{B}$, defined as

$$
T_{B}=\frac{h v / k}{\ln [1+1 / \eta(x)]}=\frac{x T_{R}}{\ln [1+1 / \eta(x)]}
$$

where $T_{R}$ is the radiation temperature, $x=h v / k T_{R}$ is the dimensionless, redshiftindependent frequency, and $\eta(x)$ is the photon occupation number. For a Planckian spectrum, $\eta(x)$ is the familiar expression $\eta(x)=\left(e^{x}-1\right)^{-1}$, and $T_{B}=T_{R}$.

Compton scattering transfers energy between electrons and existing photons but does not create new photons. The rate of energy transfer for non-relativistic electrons is parametrized by

$$
\alpha_{0}(z)=\frac{k\left[T_{e}(z)-T_{R}(z)\right]}{m_{e} c^{2}} \sigma_{T} n_{e}(z) c
$$

where $m_{e}, T_{e}(z)$ and $n_{e}(z)$ are the electron mass, temperature, and density at redshift $z$, and $\sigma_{\mathrm{T}}$ is the Thomson cross section

$$
\sigma_{\mathrm{T}}=\frac{8 \pi}{3}\left(\mathrm{e}^{2} / \mathrm{mc}^{2}\right)^{2}
$$

Eq. 6.4 is simply the average dimensionless energy transfer per collision multiplied by the collision rate. The integral of this quantity with respect to time gives a measure of the fractional energy change from Compton scattering, characterized by the dimensionless quantity

$$
y(z)=-\int_{0}^{t(z)} \alpha_{0} d t=-\int_{0}^{z} \alpha_{0} \frac{d t}{d z} d z^{\prime}
$$

(Zel'dovich and Sunyaev 1969, Kompaneets 1957). The universe is optically thick to Compton scattering at a redshift $z_{a}$ defined by $y\left(z_{a}\right)=1$, where the value of $z_{a}$ is given by

$$
\mathrm{z}_{\mathrm{a}}=3800 \mathrm{~h}^{-1} \Omega_{\mathrm{b}}-1 / 2
$$

(Darese and De Zotri 1980). Energy releases in the range $z D C \leq z \leq z_{a}$ generate a BoseEinstein spectrum, characterized by a chemical potential $\mu(x)$ : 


$$
\eta(x)=\frac{1}{e^{x+\mu(x)}-1}
$$

A Planckian spectrum is a special case of a Bose-Einstein spectrum with $\mu=0$. Bremsstrahlung creates new photons, attenuating the chemical potential exponentially at low frequencies from its value $\mu_{0}$ at high frequencies:

$$
\mu(x)=\mu_{0} \exp \left(-\frac{2 x_{1}}{x}\right)
$$

(Sunyaev and Zel'dovich 1970). The chemical potential is related to the magnitude of the energy release $\delta \mathrm{E}$ (relative to the energy $\mathrm{E}_{0}$ stored in the unperturbed radiation) by the relation

$$
\mu_{0}=1.4 \frac{\delta E}{E_{0}}
$$

The frequency $x_{1}$ is the transition frequency between a Planckian and Bose-Einstein spectrum, the lowest frequency for which Compton scattering can remove newly-generated bremsstrahlung photons to higher frequencies at $z=z_{a}$. Danese and De Zotti (1980) estimate $x_{1}$ as

$$
x_{1}(z)=67 g(x)^{1 / 2} \Omega_{b}-1 / 2 z^{-3 / 4}
$$

for $z \sim z_{a}$, where $g(x)$ is the Gaunt factor. The brightness temperature reaches a minimum at $x=2 x_{1}$. After $z_{a}$, Compton scattering ceases to sweep bremsstrahlung photons to higher energy. Free-free processes continue until recombination, effectively attenuating the initial spectrum and regenerating a Planckian spectrum at frequencies below

$$
x_{1}(z<z a)=\Omega_{b} g(x)^{1 / 2}\left[\left(z+z_{e q}\right)^{1 / 2}-\left(z_{e q}+z_{r e c}\right)^{1 / 2}\right]^{1 / 2}
$$

(Danese and De Zotti 1978). The photon occupation number evolves from Eq. 6.6 to a form

$$
\eta(x)=\eta_{a} e^{-y B(x)}+\frac{1-e^{-y_{B}(x)}}{e^{x}-1}
$$

where $\eta_{a}$ is the Bose-Einstein occupation number and $y_{B}(x)$ is the optical depth to bremsstrahlung calculated from the current epoch to the latest epoch $\mathrm{z}_{\mathrm{B}}$ at which Compton scattering could sweep bremsstrahlung photons to higher energy, $\mathrm{z}_{\mathrm{B}}=1.2 \times 10^{4} \Omega_{\mathrm{b}}{ }^{-1}$ (Danese and De Zotti 1978). Since the initial energy release occurred before the universe 
became optically thin to Compton scattering, $z_{h}>z_{a}$, Eqs. $6.7-6.9$ are insensitive to the redshift $z_{h}$ of the release,.

The CMB spectrum evolves along similar lines for energy releases after $\mathrm{z}_{\mathrm{a}}$. For $\mathrm{z}<\mathrm{z}_{\mathrm{a}}$, the universe is optically thin to Compton scattering $(y<1)$, which cannot produce the equilibrium Bose-Einstein spectrum. The result is a partially comptonized spectrum, with occupation number given by

$$
\eta_{c}(x, y)=\frac{1}{e^{x}-1}\left\{1+y \frac{x e^{x}}{e^{x}-1}\left[\frac{x}{\tanh (x / 2)}-4\right]\right\},
$$

valid for $\mathrm{yx}^{2}$ « 1 (Danese and De Zotti 1978). As before, bremsstrahlung attenuates this distorted spectrum and adds a source term at low frequencies. At low enough frequencies $x_{B}$, the universe is optically :hick to bremsstrahlung.

$$
x_{B}=2.9 \times 10^{-5} 2_{b}^{2} g(x) x^{2}\left[\left(z+z_{e q}\right)^{1 / 2}-\left(z_{r e c}+z_{e q}\right)^{1 / 2}\right]
$$

The resultant occupation number h:s a form similar to Eq. 6.9,

$$
\eta(x)=\eta_{c} e^{-y B\left(x_{e}\right)}+\frac{1-e^{-y B}\left(x_{e}\right)}{e^{x}-1}
$$

with $\eta_{c}(x)$ taken from Eq. 6.10 and $x_{e}$ calculated from the electron temperature $T_{e}$. The magnitude of the energy release is related to the Compton $y$-parameter by

$$
\begin{aligned}
\frac{d E}{E} & =e^{4 y}-1 \\
& \approx 4 y \quad(y \ll 1)
\end{aligned}
$$

(Sunyaev and Zel'dovich 1980). Compton-distorted spectra may be summarized as follows according to the redshift $z_{h}$ of the release.

- $\mathrm{z}_{\mathrm{h}}>\mathrm{z}_{\mathrm{DC}}$ : Radiative Compton scattering thermalizes any release. No observable distortion remains.

- $z_{D C}<z_{h}<z_{a}$ : Single Compton scattering establishes a Bose-Einstein spectrum characterized by a chemical potential $\mu_{0} \sim 1.4 \delta \mathrm{E} / \mathrm{E}_{0}$ (independent of $z_{h}$ ). The spectrum rises at high frequencies, reaches a minimum at $x=2 x_{1}$, and thermalizes to a Planckian spectrum at low frequencies. The drop in brightness temperature at $2 x_{1}$ is the largest observable feature. 
- $\mathrm{z}_{\mathrm{a}}<\mathrm{z}_{\mathrm{h}}<\mathrm{z}_{\mathrm{rec}}$ : The spectrum is evolving toward, but can not reach, a Bose-Einstein spectrum. The resultant partial comptonization is characterized by the redshift $z_{h}$ and the Compton y parameter, with $\delta E / E_{0} \approx 4 y$. The spectrum rises sharply at high frequencies, falls to a broad minimum in the Rayleigh-Jeans region, and rises at very low frequencies. The temperature in the Rayleigh-Jeans regime is related to the undistorted temperature by $T_{R J}=T_{R}(1-2 y)$.

- $z<z_{\text {rec }}$ : The spectrum is similar to the previous case, with negligible rise from bremsstrahlung. Compton processes arise only in the ionized matter; hence, only energy releases large enough to ionize the matter are observable. For very recent releases $(z \leq 8)$, the number of scatterings per photon is small and energy is not transmitted efficiently to the CMB.

Although Compton-distorted spectra are fairly natural in that they do not depend on the details of the energy release, they are by no means the only processes capable of producing observable distortions. The recent detection of excess flux in the sub-mm CMB spectrum revived interest in theories of greybody dust emission from an early (Pop III) generation of stars. In these models, UV radiation from an assumed population of very massive stars is thermalized by dust and re-emitted at the equilibrium dust temperature. Since the stars form well after recombination, the re-emitted photons are simply redshifted to the present era, adding an excess to the Wien region but leaving the Rayleigh-Jeans spectrum unperturbed. The exact form of the distortion depends on the epoch $z_{h}$ of the emission, the dust opacity $\kappa_{v}$, and the energy released by the Pop III stars (Adams et al. 1989, Hayakawa et al. 1987)

Another process capable of adding photons to the CMB is the decay of massive longlived particles in the early universe. Such processes will add excess photons at a frequency dependent on the mass, lifetime, and decay mode. If the decay is sufficiently early, bremsstrahlung and Compton-scattering will further distort the excess (Silk and Stebbins 1983). More exotic possibilities include vacuum decay of the universe (Bartley and Silk 1989, Freese et al. 1987) and superconducting cosmic strings (Ostriker and Thompson 1988). These models have the general features of Compton y-distortions, with a large rise in the brightness temperature at very low frequencies. In such constant-energy source models, the universe may not go through recombination. 


\subsection{Comparison of Data to Theory}

Within the past year, the greatest theoretical activity has surrounded the sub-mm CMB excess of Matsumoto et al. (1988). Their data show a clear deviation from a Planck spectrum in the Wien region. Largely for technological reasons, the CMB spectrum is much better sampled in the Rayleigh-Jeans region. The results of this thesis at 3.0 and 4.0 $\mathrm{cm}$ fall well within the Rayleigh-Jeans regime; therefore, I shall concentrate on implications of the low-frequency data on the CMB spectrum.

\subsubsection{Dust Emission}

The sub-mm excess can be modelled by red-shifted emission from warm dust at redshifts $40 \leq z_{h} \leq 20$. The main constraints on such theories are energetic: the energy density in the sub-mm excess is $-20 \%$ of the energy density in the CMB. Adams et al. (1989) find that models with $\Omega$. between 0.1 and 0.2 can explain the observed excess, where $\Omega_{*}$ is the baryon energy density tied up the Pop III stars. The large baryon density required is uncomfortably close to limits on the total baryonic density, $\Omega_{b} h^{2} \leq 0.2$, set by standard models of nucleosynthesis. The effecc of the Rayleigh-Jeans data on such models is small. The value of the CMB temperature in the Rayleigh-Jeans (sufficiently far from the distortion to be uncontaminated by it) determines the energy density of the $\mathrm{CMB}$ and hence the fractional energy in the sub-mm. The new results at 3.0 and $4.0 \mathrm{~cm}$ suggest a slightly lower temperature, and make the energetics of the excess slightly more severe.

\subsubsection{Compton y-Distortions}

The general shape of the sub-mm excess also suggests a Compton $y$-distortion. Since such models predict the Raleigh-Jeans temperature to be lowered by an amount $\delta T_{R J}=T_{R}(1-2 y)$, the quality of the fit depends critically upon the Rayleigh-Jeans temperature. Table 6.3 lists the fitted values for $y$ and $T_{0}$ for various values of $z_{h}$. The best $\chi^{2}$ for these models occurs for an energy release at $z_{h} \sim 10^{3}$, and yield values

$$
\begin{aligned}
T_{R} & =2.818 \pm 0.019 K \\
y & =0.020 \pm 0.010 \\
\chi^{2} & =34.4(16 \text { DOF })
\end{aligned}
$$


where the uncertainties are at the $95 \%$ confidence level $\left(\chi^{2}=\chi^{2}\right.$ best +4$)$. The significance of the values for $T_{R}$ and $y$ results entirly from the measurements in the Wien region of the spectrum; if only points with $\lambda \leq \lambda_{0}$ are considered, the values drop to

$$
\begin{aligned}
T_{R} & =2.789 \pm 0.022 K \\
y & =0.009 \pm 0.010 \\
\chi^{2} & =22.1(14 \mathrm{DOF})
\end{aligned}
$$

consistent with a Plank spectrum. Compton-distorted spectra also face a problem of energetics. Recent energy releases from $3 \leq z_{h} \leq 6$ have been considered as possible progenitors of the diffuse $X$-ray background, but require $\Omega_{b}>0.2$ (Guilbert and Fabian 1986). Non-baryonic energy sources would escape this problem, and could provide observable signatures at very long wavelengths.

\subsubsection{Compton $\mu$-Distortions}

Energy released at redshift $z_{h}>z_{a}$ will result in a distorted Bose-Einstein spectrum. Table 6.4 lists fitted values for $\mu_{0}$ and $T_{R}$. Although the fitted values are not consistent with a Planck spectrum, the model does a very poor job of describing the data $\left(\chi^{2}-200\right)$. The sub-mm excess is not well-described by a Bose-Einstein distribution.

\subsubsection{Distortions at Long Wavelengths}

A large fraction of the $\chi^{2}$ in various models fitting the sub-mm distortion is attributable to the discrepancy between ground-based and other techniques in the RayleighJeans portion of the spectrum. It is instructive to re-examine various models of CMB distortion to test whether these measurements can be reconciled with each other.

For all data to be consistent within their error bars would require a drop in CMB brightness below $-1 \mathrm{~cm}$, characteristic of Compton $\mu$-distortions. The data are consistent with this interpretation. Table 6.5 lists fitted parameters and $\chi^{2}$ of various models for the data set below $\lambda_{0}$. The best fit to the data results from a $\mu$-distortion with

$$
\begin{gathered}
T_{R}=2.789 \pm 0.026 \mathrm{~K} \\
\mu_{0}=0.005 \pm 0.002 \\
\chi^{2}=18.9(14 \mathrm{DOF}) .
\end{gathered}
$$


The uncertainties are $95 \%$ CL limits. The required energy release is given by Eq. 6.8 , and amounts to $0.4 \pm 0.1 \%$ of the energy in the CMB. Figure 6.2 shows the data along with the best-fitted mu-distortion. Significantly, the $\chi^{2}$ of this model is no longer attributable to a single method of measurement. However, the drop in brightness temperature is produced solely by ground-based measurements and has not been tested by a method with different systematics. In a field with a history of unsispected systematic effects, confirmation of any suspected spectral feature should be obtained by as many methods as possible.

\subsection{Conclusions}

The long-wavelength results reported in this thesis have improved the characterization of the Rayleigh-Jeans portion of the CMB spectrum. With the new results at 3.0 and 4.0 $\mathrm{cm}$, there exists an apparent discrepancy of $0.14 \mathrm{~K}$ between ground-based measurements of the CMB and measurements made using other techniques or observing platforms. This discrepancy can be resolved in two ways: either one set of measurements has undetected systematics at the $0.14 \mathrm{~K}$ level, or the spectrum of the $\mathrm{CMB}$ below $1 \mathrm{~mm}$ wavelength could contain a spectral feature such as a Compton $\mu$-distortion.

The best model of the measured CMB spectrum below $1 \mathrm{~mm}$ wavelength requires an energy release $d E / E=0.004 \pm 0.001$ at redshifts $z_{h} \geq 3800 h^{-1} \Omega_{b}{ }^{-1 / 2}$. The required CMB temperature at shorter wavelengths $(<1 \mathrm{~cm})$ is $2.789 \pm 0.026 \mathrm{~K}(95 \% \mathrm{CL})$.

At much shorter wavelengths, the measured CMB spectrum rises sharply. This can be fitted by various dust models (e.g., Adams et al. 1989) or by a Compton y-distortion with $T R=2.818 \mathrm{~K}$ and $\mathrm{y}=0.020$. This model predicts a flatter spectrum below $1 \mathrm{~cm}$ and is in marginal agreement with the data in the Rayleigh-Jeans region.

Since models such as dust emission do not alter the long-wavelength CMB spectrum, the entire spectrum can be adequately modelled by an early energy release at $z_{h} \geq 4 \times 10^{4}$ followed by a later, much larger release responsible for the sub-mm distortion. One is not forced to infer large undetected systematics in any of the current CMB measurements, although this remains a possible explanation for the low values of $\mathrm{CMB}$ measurements below $10 \mathrm{GHz}$. 


\begin{tabular}{cccccc}
\hline & $z_{h}$ & $T_{R}$ & $y$ & $\chi 2$ & DOF \\
\hline \multirow{3}{*}{$\Omega_{b}=1.0$} & $10^{5}\left(z_{a}\right)$ & 2.807 & 0.016 & 112.2 & 16 \\
& $4 \times 10^{4}$ & 2.808 & 0.017 & 89.9 & 16 \\
& $10^{4}$ & 2.811 & 0.019 & 58.6 & 16 \\
& $4 \times 10^{3}$ & 2.815 & 0.020 & 45.7 & 16 \\
& $10^{3}$ & 2.818 & 0.020 & 34.4 & 16 \\
$\Omega_{b}=0.1$ & $3 \times 10^{2}$ & 2.818 & 0.020 & 34.4 & 16 \\
& $4 \times 10^{4}\left(z_{a}\right)$ & 2.818 & 0.020 & 35.5 & 16 \\
& $10^{4}$ & 2.818 & 0.020 & 34.7 & 16 \\
& $4 \times 10^{3}$ & 2.818 & 0.020 & 34.7 & 16 \\
& $10^{3}$ & 2.818 & 0.020 & 34.4 & 16 \\
\hline
\end{tabular}

Table 6.3: Results of fitting Compton y-distortions to all recent spectral measurements.

\begin{tabular}{ccccc}
\hline & $T_{R}$ & $\mu_{0}$ & $\chi 2$ & $D O F$ \\
\hline$\Omega_{b}=1.0$ & $2.920 \pm 0.022$ & $0.028 \pm 0.003$ & 180.4 & 16 \\
\hline$\Omega_{b}=0.1$ & $2.896 \pm 0.020$ & $0.010 \pm 0.002$ & 205.7 & 16 \\
\hline
\end{tabular}

Table 6.4: Results of fitting Compton $\mu$-distortions to all recent spectral measurements. Uncertainties are $95 \%$ CL estimates.

\begin{tabular}{lccccc}
\hline Model $\left(\Omega_{\mathrm{b}}=0.1\right)$ & $\mathrm{T}_{\mathrm{R}}$ & $\mu_{0}$ & $\mathrm{y}$ & $\chi 2$ & $\mathrm{DOF}$ \\
\hline$\mu$-distortion & $2.789 \pm 0.026$ & $0.005 \pm 0.002$ & -- & 18.9 & 14 \\
\hline $\mathrm{y}$-distortion & $2.789 \pm 0.028$ & $-\ldots$ & $0.01 \pm 0.01$ & 22.1 & 14 \\
\hline Weighred Mean & $2.766 \pm 0.024$ & $\ldots$ & -- & 32.1 & 15 \\
\hline
\end{tabular}

Table 6.5: Results of various models for all data with $\lambda \leq \lambda_{0}$. Uncertainties are $95 \% \mathrm{CL}$. 


\subsection{Future Experiments}

A second possible CMB distortion would be of great interest. At present, however, the evidence for such a distortion is not overwhelming, as it rests upon statistical analysis of a number of measurements with uncertainties comprising a fair fraction of the signal. All measurements below $\lambda_{0}$ are within $3 \sigma$ of an undistorted spectrum, in distinction to the large deviations reported at sub-mm wavelengths. Since the Berkeley/Italy collaboration is responsible for all recent $\mathrm{CMB}$ measurements below $10 \mathrm{GHz}$ where a $\mu$-distortion is greatest, one cannot rule out the possibility that undetected systematics, not the CMB spectrum, are the cause of the apparent decline in brightness temperature. As long as the question of the CMB temperature in the Rayleigh-Jeans region remains open at the current level, competing models of the sub-mm excess (e.g., dust emission and Comptonscattering) will not be subjected to sufficiently stringent experimental tests.

An independent measurement of the $\mathrm{CMB}$ spectrum below $15 \mathrm{GHz}$ would be of great use. The FIRAS experiment on the COBE satellite will measure the spectrum above $1 \mathrm{~cm}$, which should confirm the sub-mm excess but will not shed further light on the RayleighJeans spectrum. In November 1989, the Berkeley group in collaboration with G. Sironi of Milano will make $\mathrm{CMB}$ measurements from the South Pole at frequencies 0.8, 1.5, 2.5, $3.8,7.5$, and $90 \mathrm{GHz}$. The measurements at 1.5 and $0.8 \mathrm{GHz}$ in particular will test models of $\mu$-distortions, as they occur near the maximum predicted distortion from a Planck spectrum (Figure 6.2). The lower foregrounds in terms of RFI, atmospheric magnitude and variability, Galactic profile, and horizon from a polar site will allow a reduction in systematics from previous measurements; however, these experiments cannot be considered independent of other ground-based results.

An CMB measurement in the range $1-10 \mathrm{GHz}$ from a balloon platform is an attractive possibility. Such a measurement would preclude the necessity of performing tip scans to measure $T_{A, A t m}$, and would avoid a potential source of systematic uncertainty (e.g., from antenna near-field response to $\left.T_{A, G r o u n d}\right)$. The frequency would be chosen to maximize the expected signal difference between a distorted and a Planckian spectrum, in terms of the achievable error (effectively trading off between the larger galactic signal and the larger distortions near $1 \mathrm{GHz}$ ). 


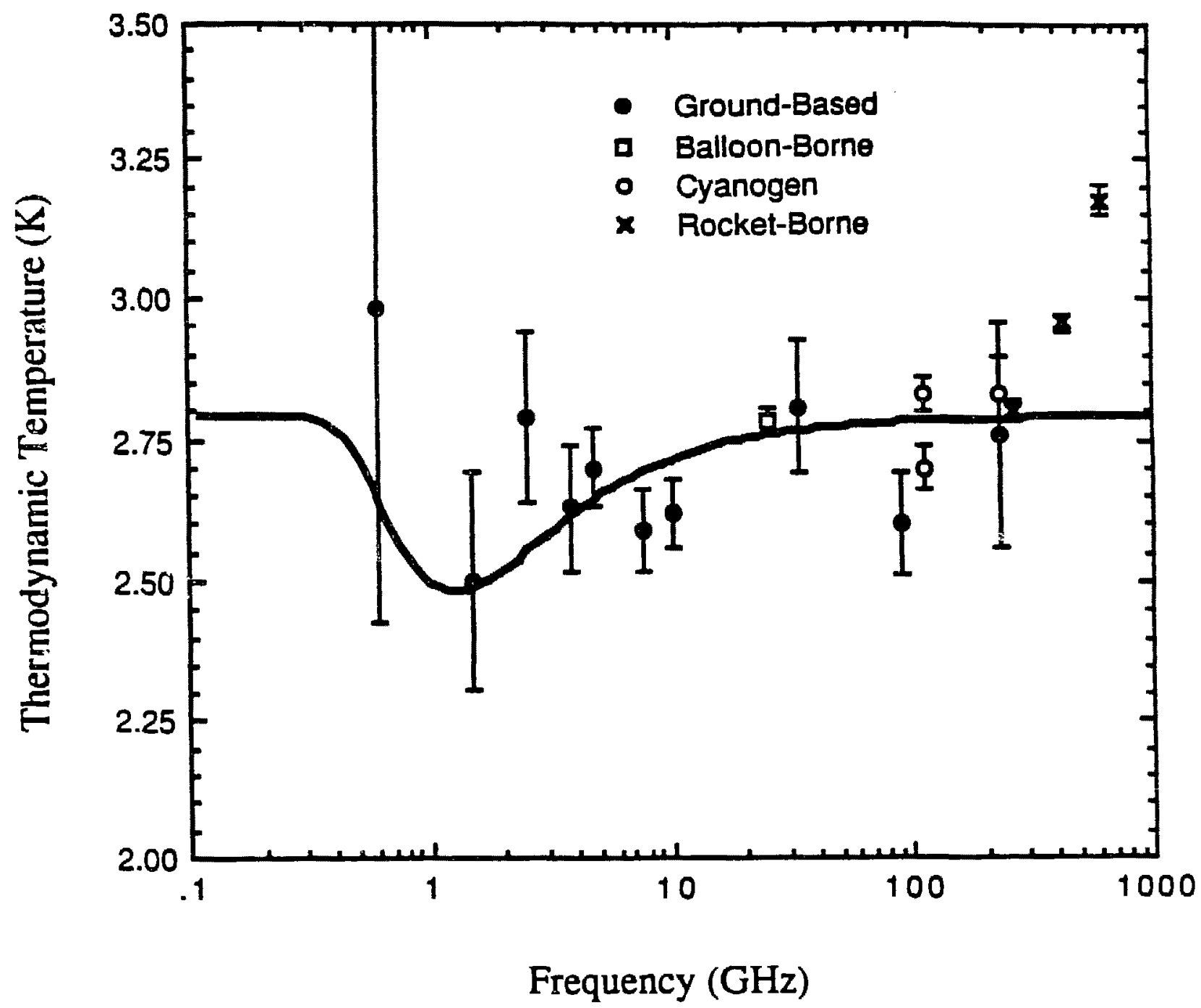

Figure 6.2: Compton $\mu$-distorion fitted to $\mathrm{CMB}$ measurements below $\lambda_{0}$. 
THE TEMPERATURE OF

THE COSMIC MICROWAVE BACKGROUND RADIATION

AT A FREQUENCY OF $10 \mathrm{GHZ}$

A. Kogut, M. Bersanelly, G. De Amici, S. D. Friedman, ${ }^{1}$ M. Griffith, ${ }^{2}$

B. Grossan, ${ }^{2}$ S. Levin, G. F. Smoot, and C. Witeasky

\author{
Space Sciences Laboratory \\ and \\ Lawrence Berkeley Laboratory \\ University of Califomia \\ Berkeley, CA 97420
}

\begin{abstract}
We have measured the temperature of the cosmic microwave background radiation (CMBR) at a frequency of $10 \mathrm{GHz}$ (wavelength $3.0 \mathrm{~cm}$ ) as part of a larger effort to determine the opectrum of the CMBR in the Rayleigh-Jeans region. The instrument used is a superbeterodyne Dicke-switched radiometer. We bave repeated the measurement over four summers with successively improved techniques and equipment. Our beat estimate of the CMBR thermodynamie temperature at $10 \mathrm{GHz}$ is $2.61 \pm 0.06 \mathrm{~K}$, where the error entimate is a $68 \%$ confidence level limit. Subject headings. cosmic background radiation
\end{abstract}

'Now at the University of California, San Diego.

${ }^{2}$ Now at the Masaschusetws Institute of Technology. 


\section{INTRODUCTION}

The spectrum of the comic micromave background radiation (CMBR) constrains energyreleaving processes in the early universe (Danese and De Zotti 1977). Energy injected into the CMBR (from turbulent proceses, particle decay, or other mechanisms) distorts the spectrum from a Planckian distribution. If the energy transfer oceurs at a redshift los than a fen times $10^{6}$, there in insuffeient time for the radiation field to thermalize to a new Planckian distribution; - combination of Compton ocattering and bremsatrahlung results in a distorted (non-thermal) CMBR spectrum (Sunyaev and Zel'dovich 1970; Danese and De Zotti 1980). The existing data allow large (200 $\mathrm{mK})$ deviations in the long-wavelength Rayleigh-Jeans portion of the spectrum (Daneve and De Zotti 1978).

To provide better measurements of the Rayleigh-Jeans portion of the spectrum, we entered un international collaboration in 1979, the reults of which have been reportad elewhere (Smoot et al. 1983, 1985a). The $10 \mathrm{GBz}$ radiometer deacribed bere was a part of that collaboration, and Look data in 1982 and 1983. In 1984, the Berkeley group modified the $10 \mathrm{GHz}$ radiometer to allor atomated atmonpheric meanurements. In 1986, the radiometer was used once again, with furtber modifieations to reduce aystematic erross observed in previous years. This paper deacribes the 10 GHz radiometer and the renult of menuremente taken with it between 1982 and 1986.

\section{EXPERIMENTAL CONCEPTS AND DESIGN}

The output of a radiometer is proportional to the power $P$ received by the antenna within a finite bandwidth $B$. The radiometer is calibrated in unito of antenna temperature $T_{A}$, which in related to the thermodynamic temperature $T$ of a blackbody completely filling the antenna aperture by the relation

$$
T_{A}=\frac{P}{E B}=\frac{2}{\left(e^{2}-1\right)} T
$$

where $z=h \nu / k T, \nu$ is frequency, $h$ is Planck's conatant, and $k$ is Boltzmann's constant.

The antenas temperature of the zenith sky, $\mathcal{T}_{\boldsymbol{A} \text {, wnith }}$, is measured by comparing the output of 
the sadiometer vieming the zenith aky to the output viewing a load of known temperature, $T_{A, 10 a d}$ :

$$
T_{\text {A,menth }}-T_{\text {A,loed }}=G\left(V_{\text {moith }}-V_{\text {lood }}\right)
$$

where $G$ is the calibration coefficient (inverse $g$ ain) of the radiometer and $V_{\text {zenith }}$ and $V_{\text {lond }}$ are the output voltages of the radiometer viewing the zenith or load, respectively. The sky signal is the sum of signals from the CMBR, the atmosphere, the galaxy, and extraneous ground radiation entering through the antenna sidelobes, where we have neglected (for purposes of this discusaion but not in the actual analysis) the small attenuation of the CMBR signal by the atmophere:

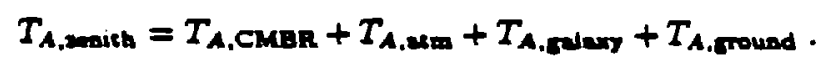

The antenas temperature of the CMBR in the reaidual after the atmoopheric, ground, and galactic aigala bave been aubtracted from the messured zenith sky temperature:

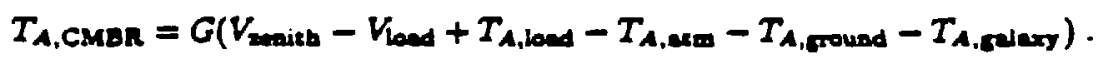

We have chocen a wavelength of $3.0 \mathrm{~cm}$ to minimize the two largeat residual signals, atmouphezic and galactic emianion. Figure 1 show galactic and atmospheric emisoion spectra for lon frequencies at a high-altitude nite. Galactic emineion is bighly anisotropic, with a maximum in the galactic plane. At low frequencies it is dominated by thermal (Hur emission) and non-thermal (synchrotron) radiation, and rives steeply at lower frequencies. Atmospheric emission at $10 \mathrm{GHz}$ is primarily from oxygen continuum; a small component is due to residual water vapor. On time males of a few hours, only the component due to water vapor tuetuates; observing from a dry, bigh-altitude site reduce both the water vapor content and variability. Accordingly, we have performed all measurements from the Nello Pace Laboratory of the Barcroft Facility, White Mountain Research Station of the University of California, situated at an altitude of 3800 meters in the White Mountains of eastern California (latitude $38^{\circ}$ ). The site lies in the rain shadow of the Sierra Nevada range and ha typical water vapor column density below $0.5 \mathrm{~g} \mathrm{~cm}^{-3}$. Atmosperic signal magnitude and variability are reduced by a factor of three compared to measurements taken 
near sea level in Berkeley, California.

To reduce systematic errors as much as possible, we measure the contribution of the atmouphere (which constitutes more than $99 \%$ of the last three terms in Equation 2) with the ame inatrument ued to meanure the zenith sky temperature. By proper design of the experiment, remaining oystematic uncertainties in the result may be reduced to a low level.

We measure the first four quantities of Equation 2 (zenith sky, cold load, atmospheric, and ground signale) at nearly the same time and with the same instrument. Only the small galactic contribution is modeled, from meanurements at lower frequencies appropriately scaled to $10 \mathrm{GBz}$. The galuctic model agree well with meaurements at $10.4 \mathrm{GBz}$ (Davies at al. 1987) and with upper limite at $10 \mathrm{GHz}$ (thil work).

The cryogenically cooled reference target, shown in Figure 2, has been described elsewhere (Smoot et al. 1983) and is shared with other radiometen. It conaists of a micronave absorber in a large $(0.7 \mathrm{~m})$ open-mouth dewar covered by two windows of 23-micron polyethylene film. The absorber is submerged beneath ambient-presure liquid helium (LHe) and acto as a microwave blackbody in an overnized multimode waveguide. The belium boiling temperature in calculated from the measured ambient barometric presure and converted to antenna temperature. Emission from the polyethylene windowe contributes $5 \pm 2 \mathrm{mK}$; resistive lowes in the wallo contribute $9 \pm$ $5 \mathrm{mK}$; the power emitted by the radiometer and reflected by the absorber contribute $7 \pm 4 \mathrm{mK}$ (Friedman 1984). The coberence length of the radiometer is small enough that coherent reflection is not a problem; coberent reflections from the target and the radiometer contribute $0 \pm 10 \mathrm{mK}$ to the wotal load signal. Summing these signale, the antenna temperature of the cold load is

$$
\begin{aligned}
T_{\text {A,load }} & =3.562 \pm 0.013 \mathrm{~K}(1982-1984) \\
& =3.568 \pm 0.013 \mathrm{~K}(1986)
\end{aligned}
$$

The highet value for 1986 is the result of a slightly higher ambieat presure in the cold load that year.

We measure the zenith sky temperature $T_{A, \text { andith }}$ by comparing the output of the radiometer as 
it alternately views the cold load and the zenith sky. At $10 \mathrm{GHz}$ on White Mountain, the zenith sky is within $100 \mathrm{mK}$ or the cold losd, which greatly reduces the dependence of the CMBR result on precise knowledge of the abeolute calibration.

We measure the atmospheric temperature $T_{A, \text { and }}$ by correlating the aigal change with the air mas in the beam a the radiometer beam ccane different zenith angles. Uaing the same radiometer to meanure both the atmoopheric and zenith aky temperatures eliminates a large source of potential oystematic uncertainty. We further reduce uncertainty in the atmoupheric reaults by menuring the atmosphere in two independent ways and correlating the resulto with atmoopherie meanurements performed at the eame time at other frequencies.

We calibrate the radiometer by comparing ito output an it alterately views targets of known, disimilar temperature. The use of targets at widely different temperatures (LHe and ambient) reduces the precivion to which each temperature must be known. A third, intermediatetemperature target (liquid aitrogen, LN) allows the amall saturation effects to be calculated. The calibration is monitored to better than $1 \%$ throughout the meanurement.

The use of a Dicke-awitebed radiometer viewing similar-temperature targets in elose succession reduce the effects of absolute calibration uncertaintie and drifu to low levels. A calibration uncertainty of $1 \%$ corresponds 10 an uncertainty of $12 \mathrm{mK}$ in the atmospheric temperature and les than $1 \mathrm{mK}$ in the zenith sky temperature. Proper use of a Dieke-switched zadiometer require atable, cold reference target. We use the senith sky for a reference, either viewed directly (primary antenna) or reflected by an aluminum mirror (secondary antenna). The radiometer is designed to allow the two antenna beams to scan independently.

\section{DESCRIPTION OF TBE RADIOMETER}

The radiometer used throughout the experiment in s superheterodyne Dickeswitched radiometer with a pasuband from 9.5 to $10.5 \mathrm{GBz}$, shown schematically in Figure 3. The two input porte are identical, conical, corrugated born antennas, manufactured by CSELT of Torino, Italy, and deaigned to have low sidelobes. The measured balf-power beamwidth is $12: 5$, symmetric in 
the E- and B-plenes (Bielli et al. 1983).

The aignal received by the antenna is fed through rectangular waveguiden to an electromagnetic switch consisting of a latching ferrite tbree-port circulator. The switcb has a meanured insertion low of leas tban $0.3 \mathrm{~dB}$ between any two coupled ports and han greater than 25 dB ieolation between uncoupled ports. It is switched at $100 \mathrm{~Hz}$ by an external clock signal. The circulator allows the input horns to act as sky horns: when the switch connects one horn to the radiometer, power emitted by the radiometer is broadcast out the other horn, reducing reflection effects. An isolator located between the switch and the mixer further reduces the magnitude of the broadcast power. The measured isolation is greater than $36 \mathrm{~dB}$.

The Gund-efect local acillator (LO) has output power of approximately $18 \mathrm{~mW}$, which is reduced to approximately $10 \mathrm{~mW}$ by a waveguide attenuator. The $\mathrm{LO}$ is tuned to $10.00 \pm 0.01$ GHz as confirmed by a spectrum analyzer.

The mixer and intermediate-frequency (IF) pre-amplifier are a single unit witb nominal bandpaes 45-550 $\mathrm{MBz}$ and direct RF to $\mathrm{IF}$ gain of $24 \mathrm{~dB}$. The difference frequency between the LO and sky signal, after preamplification, undergoes an additional $41 \mathrm{~dB}$ of amplification in a eecond IF amplifier with oominal bandpae 5-500 MEz. A 6 dB attenuator between the two amplifiess serve to decouple them and reduces non-linear aturation in the radiometer. The signal is reetified by a Sebottky-barrier detector diode and fed to a lockin amplinier. The lockin consist of a demodulator synehronow with the switch and an ideal integrator which averages the demodulated signal for a 2-eccond period. The voltage gain of the lockin is approximately $1.5 \times 10^{4}$. The aignal is then digitized and recorded on magnetic tape.

The renaitivity of a Dickeswitched radiometer is defined as the input temperature difference $\Delta T$ which give rise to an output voltage equal to the RMS thermal goise fluctuations of the radiometer icself. For a system with equarewave switching and wide band detection this is given by (Kraus 1966)

$$
\Delta T=2 \frac{T_{\mathrm{ny}}}{(B T)^{1 / 2}}
$$


where $T_{\text {oys }}$ is the system noise temperature, $B$ is the IF bandwidth, and $r$ is the post-detection integration time. For this aystem, $T_{0 \mathrm{n}}=490 \mathrm{~K}, B=455 \mathrm{MHz}$, which gives $\Delta T=46 \mathrm{mK}$ for an integration time $r=1$ second. The meaured value is $46 \pm 3 \mathrm{mK} \mathrm{Hz}^{-1 / 2}$

All receiver components from Dicke switch onward are enclosed in RF-shielded, thermallyinsulated boxes, as are the power supplies for the receiver and asociated electronics. As the calibration coeffeient of the radiometer is rencitive to changes in the temperakure of the receiver components, thermal control cireuits regulate the temperature of the plate and the power-supply bax to within $0.1 \mathrm{~K}$. We also monitor the temperatures of the Dicke switeh, the power-supply box, boch antennas, and the plate containing the mixer, IF amplifien, and detector diode. The borns are too large and exposed to regulate; bowever, temperature drifts in the borns are slow compared to the period over which a single measurement of the CMBR is made ( 160 to 256 seconds).

The radiometer is mounted on bearings and is free to rotate about a borizontal axis coincident with the aymmetry axis of the secondary horn. The primasy horn, mounted at right anglea, may rotate though $360^{\circ}$. Stops on the bearing mounte allow the primary to point at angles of $\pm 30^{\circ}$, $\pm 40^{\circ}, \pm 90^{\circ}$ degree from the zenith, directly at the zenith $\left(0^{\circ}\right)$, or directly downward $\left(180^{\circ}\right.$, the position of the cold load). A mirror mounted at $45^{\circ}$ wo the mecondary born axis redirects the secondary beam to the aky.

The finite conductivity of the aluminum mirror contributes a small linearly polarized component to the signal received by the secondary horn. The rotation of the radiometer with rapect to the fixed mirror then induce an angledependent modulation of the reference signal. To seduce the magnitude of the modulation, a quarter-wave plate in installed in the throat of the secondary born. The quarter-wave plate coniste of a thin Teflon card, which createa a phase difference of $90^{\circ}$ between the eomponents of incident radiation polarized along and across the card. Mounted at $45^{\circ}$ to the rectangular waveguide, it bas the effect of transforming linearly polarized radiation aligned with the rectangular waveguide into left-circularly-polarized radiation, and vice veras. The secondary arm of the receiver is then sensitive to lef-circularly-polarized radiation and io insensitive to the orientation of incident linearly polarized radiation. The quarter-wave plate 
is uot perfect, and a small residual modulation exists. The magnitude, though, is reduced from approximately $70 \mathrm{mK}$.to less than $5 \mathrm{mK}$.

The ambient calibration target consists of a slab of microwave absorber (Eccosorb CV-3) mounted in a thermally insulated box. Beld over the primary born, it completely fill the hom aperture. A temperature sensor buried within the absorber monitors the temperature to an accuracy of $0.1 \mathrm{~K}$. The box is cloced on all sides when not actually calibrating the radiometer to allow the entire abeorber to maintain thermel equilibrium.

\section{a) 1982 Configuration}

The radiometer was first assembled in 1982 . The primary born could point to zenith angles of $\pm 30^{\circ}$ while the secondary viewed the sky reflected in a large rectangular aluminum mirror. The cecondary born faced due north; the primary horn owung in an eant/west plane with negative zenith anglen weat. Ground screen constructed of wire mesh prevented extraneous ground radiation from entering the beam. One cet of ecreens surrounded the secondary horn and mirror on the aide and bottom; another set surrounded the sides of the primary born $a$ it viewed the oky (Figure 4).

We took date by rotating the primary born wo that it alternately viewed the eryogenic target buried in the ground, the zenith aky, the atmonphere at zenitb angles of $-30^{\circ}$ and $+30^{\circ}$, and finally an ambient calibration target mounted at $+90^{\circ}$. The radiometer viewed each sarget in turn for 32 seconds, then rotated to the next. Lea than 4 seconds of each 32 were spent rotating the radiometer; the rest were devoted to collecting and averaging the signal.

\section{b) 1989 Configuration}

The small differential air mass between zenith angles of $30^{\circ}$ and $0^{\circ}$ magnified the effect of systematic errors on the calculated atmospheric temperature. In 1983, we added additional zenith angles of $\pm 40^{\circ}$ to the allowed primary horn positions. This brought the tilted primary beam eloser to the borizon; to provide adequate protection againgt diffracted ground radiation, the mesh screens sbout the primary born were enlarged. The screens surrounding the secondary horn and 
mirror were also enlarged, and mechanical support for the radiometer's electronics was improved. In all other respecte, the radiometer in 1983 clonely resembled the 1982 configuration.

\section{c) 1984 Configuration}

Atmoupheric meanurements taken with the primapy horn were subject to a fairly large statistical seatter, due mostly to the small zenith angles used. In 1984, the rectangular mirror redireeting the secondary beam was replaced by a smaller elliptical mirror. This mirror rotated about an axis coincident with the wecondary horn axis and could be stopped automatically to view any desired zenith angle to within $4^{\prime}$.

Two independent methods existed to meawre the atmoapheric temperature. Meanurements with the primary anteane ("primary atmosphere") were made as part of the CMBR measurements. In addition, meaurements with "be secondary antenna ("secondary atmoapheres") were made at otber times throughout the night to provide a check on the primary resulte and to monitor the atmouphere while other radiometes performed CMBR meaurements over the cold loed. The eecondary mesurements took place on a platform located approximately $20 \mathrm{~m}$ from the cold loed, rotated $25^{\circ}$ to the weat to take advantage of the borizon profile at the platform site. Poaitive zenith angles during secondary scan bave an azimuth of $245^{\circ}$, negative zenith angles 65". Primary atmonpheric scans were changed to vien the zenith twice per scan; primary scans now comprised the angles $180^{\circ}$ (cold target), $0^{\circ},-40^{\circ},-30^{\circ}, 0^{\circ},+30^{\circ},+40^{\circ}$, and $+90^{\circ}$ (ambient target), in sequence. The secondary scanned angles of $+54^{\circ},+47^{\circ},+40^{\circ},+30^{\circ}, 0^{\circ},-30^{\circ},-40^{\circ},-$ $47^{\circ}$, and $-54^{\circ}$ in order, while the primary horn viewed the zenith sky as a convenient reference. Periodically, the scan was stopped and the radiometer calibrated with the ambient target beld over the secondary horn.

The elliptical mirror wa meanured to intercept $99.7 \%$ of the secondary beam. To prevent signal modulation arising from the remaining $0.3 \%$, a large mesh screen was erected bebind the mirror to redirect to the aky any spillover that might otherwise view the ground. The mesh cereens to the aides of the mirrop were redenigned to allow the mirror to rotate, and enlarged again to prevent difracted ground radiation from entering the redirected beam at the largeat zenith 
angles. Due to problems with the ground shields, the secondary atmospheric scans did not provide any ueful dats in 1984 .

\section{d) 1986 Configeration}

Messurements made after 1984 showed that a combination of dust and dirt collecting on tbe wire mesh ground screess and ground radiation transmitted through the screens could add a aignal of up to $10 \mathrm{~K}$ if the antenna directly viewed the sereens. While part of this was due to the aging and axidation of the wire mesh, prudence dictaled tbat we build alternate ground abields. By 1986, the menh ecreens bad been replaced by aluminum sheet ground shield, with the exception of a amall ecreen uned around the primary during secondary atmonpheric ecans. Additional ground abieldo were added; the weondary horn/mirror were non completely obielded in all direction except up. To block difracted greund radiation during secondary atmonf heric acane, a cet of exteasions to the side shields was added. The exteasions were fixed at a larger zenith angle thas the side shields and undercut them; they were designed to substitute sky radiation for diffracted ground radiation. A cer of quarter-wave traps on the original gide shields further reduced the amount of diffracted sadiation entering the secondary beam.

Data from 1984 stowed that the quarter-wave plate in the throat of the secondary born could move far from its optimal position, greatly reducing its effectiveness. Thermal strese from diurnal heating also bent the Tetlon card, again redueing its effectiveness. In 1986, a shallow groove was cut to bold the card, and the card was removed during the day. New cards made for the 1986 measurements sbowed an improvement over past years; their performance did not deteriorate throughout the aight.

Test in 1986 showed a $100 \mathrm{mK}$ signal modulation caused by a slight ( 10 arcmin) misalignment of the necondary horn's aymmetry and rotation axes. Rotating the primary horn caused the beam opillover past the elliptical mirror to vary slightly with position. An oversized trapezoidal mirror wa fitted over the elliptical mirror during primary CMBR scans. This removed the modulation.

With new confidence in the results of the secondary atmospheric gcans, we altered the 
data collection routine. Each night, secondary atmospheric measurements were made from the platform for about an hour, with all ground shields in place and a small mesh screen protecting the (reference) primary from the movement of nearby personnel. The mirror changed ponitions autometically every 32 seconds. We then carried the radiometer to the cold load, where the trapezoidal mirror was placed over the elliptical mirror and an aluminum ground abield placed around the primary. The side diffraction shields were not not needed for primary scans, where the secondary contribution aeeded only to be stable. After the usual CMBR measurements over the cold load, the radiometer was carried back to the platform, where it resumed secondary atmomperic acan until dawn. Repeated movement of tbe sadiometer did not affect it performance. Figure 5 shows the radiometer in its 1986 configuration.

\section{SYSTEM PERFORMANCE TESTS}

We made numerous teats of the radiometer and abociated apparatus before and after each trip to White Mountain to acese the magnitude of effects contributing to the error budget. These teats were performed in Berkeley during night when the atmosphere was stable enough to yield consistent results. We repeated the most critical tests at White Mountain to verify that no changes had occurred during the trip to the site.

\section{a) Systematic Ofset Change}

The CMBR measurement and atmospheric measurements made with the primary born compare data taken with the radiometer rotated to view various targets. As the radiometer rotates on its bearing, gravitational atresees on the components change. It is vital that these changing atreses do not ayatematically change the performance of the radiometer, either in offset or gan, where the offset is defined a the output signal for zero temperature difference between the two input ports. We bave tested extensively to limit this effect.

In the simpleat teat, we covered the primary hord with an ambient-temperature Eecosorb target, completely filling the aperture. The secondary viewed the reflected sky. We then looked 
for output ebanges as the radiometer rotated. This procedure tested all radiometer componenss except the primary horn upatreem of the Dicke awitch, and was quite sensitive to gain changes. To teat the primary upatream of the switch, the primary muat view a cold target. This may be either an Eecoeorb target saturated in LN, or the sky viewed directly or througb reflector. In the latter two cases, the radiometer cart direction was reversed (or the reflectors interchanged) and the teat repeated to separate instrumental aymmetries from signal aymmetries. In all cases, the radiometer was repeatedly moved between two or more positions for periods of approximately 17 minutes; the output was recorded every 32 seconds and digitally signal-averaged to improve the atatistica.

Small signal variations were observed as the radiometer primary horn rotated, but they were not consistent in sign or value from test to test. Upper limits to signal variation induced by changing radiometer position are given in Table 1 for both warm and cold targets. The lack of consistency from test to test precluded the simple interpretation of warm and cold signals in terms of a combination of gain variation and ofiset change for both targets. Instead, we preferred a more concervative spproacb and took the magnitude of the cold target resus. an an upper limit on offset changes and the varm target renult a a upper limit on gain variation. As we bave taken the largest signal change obwerved in many tests rather than the average, the result is likely to be an overestimate of the actual systematic offset change during CMBR measurements.

In all years, the gain was seen to vary negligibly as a function of radiometer position. Maximum fractional gain variation measured with Eccosorb over the primary horn was less than $10^{-3}$. Note that, for the $180^{\circ}$ (atraight dowa) position, it was imposoble to use the sky as a cold target. In this cace, piece of L.N-soaked Eccosorb was beld over the primary born and the output compared for the up and down positions. Prior to 1986, this placed an upper lime of $55 \mathrm{mK}$ on the nize of any output changes caused by radiometer rotation. The limiting factor was observed drifts in output commencing approximately 15 seconds after the L.V-saturated target was placed over the born. We asumed that this represented thermal drift in the target. Tests in 1986 with a new target revealed that the drift were caused by the rapid cooling of the primary horn induced 
by contact with LN. Tests performed with the horn temperature carefully controlled then gave a aigual change of $6 \pm 17 \mathrm{mK}$ due to horn rotation, consistent with zero change and with the results obtained with ambient Icensorb over the primary. Rapid horn cooling at White Mountain was not a problem, a the meanured horn temperature changed by lese than $1 \mathrm{~K}$ over a 40 minute observation.

\section{b) Calibration Stability}

Use of a Dicke-aritched radiometer reduces effects of calibratica variation, but does not eliminate them completely. An error of $1 \%$ in the calibration of the zadiometer causes a 12 $\mathrm{mK}$ error in the atmospheric termperature, and thus in the CMBR temperature. We measured the calibration coefficient every 256 secondo during CMBR measurements, and approximately once every 15 ninutea during secondary atmonpheric scans. We therefore required the system calibration to be stable to $1 \%$ or better for at least this long.

We measured calibration stability by placing an ambient Eccosorb target over one horn while the other viewed the aky. Drifte an amell a $\delta G / G=10^{-3}$ produced output changes larger than radiometer or atmospheric noise and mere easily observable. Maximum drift observed in all years wa lea than $3 \times 10^{-3}$ in 17 minutes, earily satisfying our requirement.

\section{c) Calibration Linearity}

We calibrated the radiometer by comparing the output as the radiometer viewed iargets of known, widely separated temperature. Saturation of the detector diode due to the large power emitted by the ambieat turget caused a slight decreace in signal from atrict linearity. For the calibration to be sceurate to $1 \%$, this effect must be meanured and included in the data analysis. Detector aon-linearity wa found two ways: by direct measurements of the detector diode, and by subetitution of an iatermediate-temperature (LN) target for the warm target. The second method bart the advantage of teating the repone of the entire detection chain, and provided the more accurate result. In this method, we alternately calibrated between the ambient target/zenith sky and a LN target/zenitb sky. LN provided a convenient target in the linear regime of the detector. 
We then messured the atmospheric signal, asoumed a zenith sky temperature, calculated the system gain, and repeated until we achieved a self-consistent result. The ratio of system gain viewing $L N$ and system gain viewing an ambient target provided the non-linear reaponse of the system. The resultiag detector saturation is shown in Table 2. The higher saturation value in 1982 was due to a faulty zener diode in the lockin amplifier, replaced after 1982; all other results were within experimental limits. Maximum calibration uncertainty was $1 \%$ in 1982 and 1983, and was negligible for 1984 and 1986.

\section{d) Integration}

The enaitivity of the radiometer, as defined in Equation 3, is inversely proportional to the square root of the obcerving time so long an the radiometer noise contains no time-dependent atructure. As a basic teat of radiometer performance, and as a planning tool to enable us to determine bow beat to use our limited observing time, we meanured the period at which the white-noise anumption broke down. In thin test, both horne viewed the same cold target (to prevent target variation from entering the analysia) while the dats were averaged for longer and longer periode. Typically the zenith aky was uned a a target. The RMS fuctuation in the data decreaced a expected for periods up to 64 eeconds, after which non-random radiometer luctuation dominated. This corresponded to an RMS noise level of $5 \mathrm{mK}$, aegligible for our purposes.

\section{e) Magnetic Sensitivity}

The Earth bes a magnetic field of approximately 0.5 Gauss. The ferrite switch and isolator are the components most sensitive to changes in the external magnetic field; an the radiometer rotatea, the changing relative orientation between radiometer components and external field could conceivably induce a position-dependent signal modulation. We minimized the effect by wrapping the awitch and isolator in several layers of mu-metal foil. To ensure that enough foil is uned to reduce the residual field to negligible levels, we erected large ( $1.5 \mathrm{~m}$ diameter) coils around the radiometer and tested for magnetic response at field strengths of 10 and 5 Gauss, oriented 
perpendicular to the rotation axis of the radiometer. The largest response (1.5 $\mathrm{mK} / \mathrm{Gauss})$ contributed negligibly to the radiometer response, and was consistent with the limits eatablished by the offeet teats.

\section{f) Polarization Response}

A quarter-wave plate in the throat of the secondary born transformed the small linear polarization of the aignal emitted and reflected by the mirpor into circular polarization. The transformation is not perfect, and a residual linearly polarized oignal causes a pooition-dependent output modulation. We evaluated the effectivenea of the quarter-wave plate by recording the response of the radiometer to a known large polarized signal an the angle between the polarization "ector and the radiometer was changed. The amal polarized aignal from the mirror ba been meaured and agreed with the expected signal within the limite of measurement. The resultant reaidual signal after inotallation of the quarter-wave plate bad a maximum amplitude of $5 \mathrm{mK}$. This wa consiateaz with the limits set by the offet teats, which included but did not reparat:? polariation effects. The effectivenee of the quarter-wave plate depended alightly on ita ponition within the cecondary born's throst. We meaured the reaponee of the plate each time it wa installed.

\section{g) Poinling}

We determined the atmospheric temperature by correlacing the total signal received at a given zenith angle with the air mas at that angle, using an stmospberic model. Botb the accuracy and repeatability of the angle uned bave beed teated many times. Prior to 1986, the primary horn was the major source of atmonperic information. The pointing was found to be accurate and repeatable to within $4^{\prime}$. In 1986 , the secondary and rotating mirror provided the most accurate stmonpheric information, while the primary provided the zenith sky measurement central to the CMER determination. Eacb time the large trapezoidal mirror wa placed over and then removed from the smaller rotating elliptical mirror, the previous absolute pointing of the rotating mirror wa destroyed. Rather thas spend the hours necesary to set the anglea back to their precise 
nominal positions, the angles were reset to within $30^{\prime}$ of the nominal positions and the sctual powitions recorded. Test then showed the actual positions chosen were repeatable to $4^{4}$ or better. We accorded similar treatment to the primary horn in the interests of saving time, using the the recorded true pointing angles in the atmospheric analysis.

\section{h) Sidelobe Reception}

Extraneous ground radiation entering the beam sidelobes or diffracting over the shields into the inner beam represented a potentially large source of error in the measurement of the atmosphere and zenith oky. We preferred to meanure the effect directly rather than rely on modele baed on beam patterns and abield/horizon profiles. We measured aidelobe reception over the abields by duraately raining and lowering large duminum abets a aimple extenvion to the exinting ground abielda. We recorded the output and looked for signal changes correlated with extension poaition. If any vigal wa obcerved, we repeated the teat with succeanively larger obields until no further ebange was een. The total signal change from the largest extension to no extensions was taken a the magnitude of the sidelobe aigna. A vimilar procedure teated for radiation diffracting over the shields, with the single exception that the additiond shields were not beld at the anme argle as the main shield, but at a shallower angle. We tested the primary horn at all angles in all years. No sidelobe signal was ever obcerved at zenitb. Small (typically $3 \mathrm{mK}$ ) signals were seen at $40^{\circ}$; theae were consistent in any given year and were ubtracted from the data at the appropriate angles. The secondary bas also been teated since its inception in 1984. Sidelobe reception of up to $20 \mathrm{mK}$ was observed in 1984 ; improved ground shields in 1986 reduced this to below $3 \mathrm{mK}$ at all angles.

\section{i) Primary Sereen Interference}

During secondary atmospheric mesurements, we placed a small mesh screen around the primary horn to prevent the movemeat of nearby persondel from affecting the refereace beam. Teste after we returned to Berkeley in 1986 showed that this screen itself caused a signal modulation dependent upon the mirror position. We measured the effect in Berkeley in two ways. 
We alternately removed and replaced the screen and cheeked for effects correlated with screen ponition. We also performed alternate atmospheric scans with and without the screen. The two methods gave reaults in agreement with each other; the effect ranged from $70 \mathrm{mK}$ at $-30^{\circ}$ to 140 mK at $+47^{\circ}$. We removed this effect from the 1986 secondary data.

\section{DATA REDUCTION AND ANALYSIS}

To mearure the antenne temperature of the CMBR, $\boldsymbol{T}_{A, C M B R}$, we independently measured the zenith sky temperature $T_{A, \text { naith }}$ and the atmonpheric temperature $T_{A, \Delta t m}$. We then obtained $T_{\text {A.CMBR by colving Equation } 2 .}$

The voltage output of the radiometer wa digitized with a 16-bit analog-to-digital converter (ADC) and recorded in digitized unite (du). Houcekeeping information (temperatures witbin the radiometer, beam pointing, ambient temperature and presoure) was also recorded. Data taken between ponitions were disearded, and the rest used for analysis. Analysis proceeded on a scanby-scan basis. First, the calibration wa calculated (for scans with a LN or LEe cold load) or estimated (for secondary atmospteric scans which use the zenith sky as a second calibration load). Corrections to the data were made, the galactic contribution removed, and the sky and atmospheric temperatures calculated. For secondary atmoopheric scans the calibration, zenith sky and atmonpheric temperatures were recalculated to obtain a self-consistent result.

\section{a) Calibration}

We measured the calibration coeficient $G$ of the system using an ambient target and a cold load:

$$
G=\frac{1}{S} \frac{T_{\text {A.amb }}-T_{\text {A.load }}}{V_{\text {amb }}-V_{\text {lowd }}}
$$

Where $S$ in the gain saturation factor (Table 2); $T_{A, a m b}$ in the antenna temperature of the ambient target; $T_{A \text {,loed }}$ is the anisana temperature of the LHe load; $V_{\text {anb }}$ is the radiometer output viewing the ambient target; and $V_{\text {lad }}$ is the radiometer output viewing the LBe load. The value of G was roughly $9 \mathrm{mK} / \mathrm{du}$ in all years, adjunted so the largest signal difference (ambient-sky, approximately $300 \mathrm{~K}$ ) remained on the \pm 32767 -du scale of the ADC. 


\section{b) Atmospheric Antenno Temperature}

If the antenna beam were a delta function, the atmosphere were a fat slab, and no sources exinted exteral to the atmouphere, the stmoupheric antengs temperature would be given by

$$
T_{A, \text { atm }}=G \frac{V_{0}-V_{\text {zenith }}}{\sec (\theta)-1}
$$

Where $V_{\text {anitb }}$ in the radiometer output viewing the zenith sky, and $V_{0}$ is the radiometer output at zenith angle $\theta$.

In practice, the antenge beam bas a $12: 5$ balf-power beamwidth, the atmouphere follows the eurve of the Earth, exterual radiation is attenuated differently at different angles, and bighly anieotropic cource of radiation exiat exterad to the stmosphere (e.g. the galaxy). These effects are small (the optical deptb of the atmosphere $r \approx 0.005$; a curved atmosphere deviate from a flat one by $0.2 \%$ at $40^{\circ}$ ) and partly cancel; the total differeace between Equation 4 and the atmospheric model used amounts to about $50 \mathrm{mK}$. The model used is similar to that used by Witebsky et al. (1986), with a single ambieat atmospheric temperature of $240 \mathrm{~K}$ and a single scale height of $7 \mathrm{~km}$ for $\mathrm{O}_{2}$ and $\mathrm{B}_{2} \mathrm{O}$. Uncertainty in these parameters contributes aegligibly to the atmospheric uncertainty; the dominant uncertainty in the model in the convolution of the beam pattern with the atmosphere.

The galactic signal, although lea than $30 \mathrm{mK}$ at $10 \mathrm{GHz}$, does have the potential to affect the atmonpheric calculations. In summer at White Mountain, the atmospheric scans can cut through the galactic plane. The differential galactie signal between zenith and the angle $\theta$ is multiplied by approximately $[\sec (\theta)-1]^{-1}$ in the atmonpheric calculations. At $40^{\circ}$, this triples the effect of the sigal. We subtracted the galactic oigaal from the data using galactic maps compiled from data at lower frequencies (Baslam et al. 1982) and extrapolated to $10 \mathrm{GHz}$. An error in the extrapolated values as great as 50\% would, in the worst ease, affect the messurement at one atmospheric angle by lese than $40 \mathrm{mK}$, or the mean $T_{\text {A,am }}$ by lese than $10 \mathrm{mK}$.

The automated atmospheric scane taken in 1986 provided a direct upper limit to the magnitude of the differential galactic aigaal. Data in which the galactic plane has moved through 
the beam at one angle were indistinguisbable from reference data at other angles far from the plane. This placed an upper limit of $30 \mathrm{mK}$ on the peak magnitude of the galactic signal, limited by atmonpheric drifte over several hour intervals, and connistent with model predictions of $25 \mathrm{mK}$ peak differential signals.

After corrections for galactic and minor sidelobe contributions, the data at various angles were averaged to get one value for the atmospheric temperature for each scan. In 1982 through 1984, we took $T_{\text {A,nem }}$ at each angle $\theta$ to be the mean of the values at $\pm \theta$. This procedure reduced the effects of an overall tilt in the radiometer cart to les than $10 \mathrm{mK}$, so long as the relative ceparation of the angles wa correct to $10^{\circ}$. The mean value was anigned an uncertainty; we then took s weighted average of the $30^{\circ}$ and $40^{\circ}$ reaults. The statistical and (weighted) systematic uncertainties were added in quadrature; the reaulting uncertainty wan dominated by systematic effecte, primarily limits to the systematic offet change.

In 1986, the most accurate atmospheric data came from the secondary measurements, which were not subject to an oftet change. Eight independent measurements were made each scan. We made as additional correction for the effects of the primary screen modulating the secondary signal. After correction, the data atill showed a aystematic spread of about $130 \mathrm{mK}$, with $+54^{\circ}$ consintently bigheat and $-30^{\circ}$ lowest. The effect may be due to uncertainty between the sereen modulation effect at White Mountain and an measured in Berkeley with a different horizon profile. As the spread was larger than the individual systematic errors discussed above, we did not weight each angle but instead simply took the mean of all 8 values. We took half the value of the total spread in the dats ss conservative entimate of the aystematic uncertainty and added it in quadrature with the amsli : tatintical uncertainty. The teoultant uncertainty was still smaller than the uncertainty in the prims atmospheric measurements; the atmoopheric temperatures obtained by botb methods were in excellent agreement. Table 3 shows the measured atmospheric temperature for 1982-1986.

The secondary atmospheric data were not taken simultaneously with the zenith sky measurement. $90 \mathrm{GBz}(0.33 \mathrm{~cm})$ atmospheric scans showed a smootb linear drift of atmospheric 
temperature throughout the aight. Accordingly, we obtained the $10 \mathrm{GHz}$ atmospheric temperature by linear interpolation of the secondary atmospheric data. The resulting $10 \mathrm{GHz}$ atmospheric temperatures for the timn of the zenith sky measurement are shown in Table 4, along with the reults of (noisier) primary atmonpheric scans taken during zenith sky measurements.

Comparison of atmospheric results from all four years is a useful check against possible systematic effects. Figure 6a shows the $10 \mathrm{GHz}$ atmospheric antenna temperature plotted against the atmospheric antenna temperature measured simultaneously at $90 \mathrm{GHz}$, an independent measure of the atmosphere. The 1982 values are all anomalously low, indicative of eitber anonalous atmospherie behaviour of a systematic error. In addition, the 1982 atmospherie data did not aupport the result of meanuremente taken by our collaboraton at nearby frequencies (Partridge et al. 1984). We have additional atmospheric information in the form of the zenitb sky temperature, which is roughly the sum of a constant CMBR term and a variable atmospheric term (Equation 1). Figure $6 b$ abowa the measured sky temperature at $10 \mathrm{GBz}$, also plotted aganst the $90 \mathrm{GHz}$ atmospheric temperature. The data correlate well; in particular, the 1982 result show: no sign of atmospheric nomalie at either 10 or $90 \mathrm{GHz}$. Any true atmospheric effect uhould appear in both plots; $a$ it doea not, we conclude that the previoualy reported 1982 atmoopheric temperature are too low by approximately $0.23 \mathrm{~K}$. This is easily understood if a systematic offset of $0.03 \mathrm{~K}$ were present; at zenith angles of $30^{\circ}$ used in 1982, the effect of any offset change on the atmonpherie ealculation is multiplied by more than nix. The previously quoted uncertainty of $0.16 \mathrm{~K}$ for the 1982 atmoupheric temperature in probsbly an undereatimate, an this value used the average of many offet tent ratber than the maximum reported in Table 1.

A better entimate of the 1982 atmoupheric temperature may be obtained by using the correlation between the atmospheric temperature at 10 and $90 \mathrm{GBz}$ as measured from 1983 to 1986, for which the data show no evidence of systematic effects. The best-fit line to the $1983-$ 86 atmoopheric data bae a slope $0.013 \pm 0.005$ and an intercept $1.012 \pm 0.075 \mathrm{~K}$, compared to a theoretical alope of $0.019 \pm 0.001$ and an intercept of $0.88 \pm 0.08 \mathrm{~K}$ (Costales et al. 1986). From this and the measured 1982 atmospheric tempersicice at $90 \mathrm{GHz}$, we obtain the estimated $10 \mathrm{GHz}$ 
atmospheric temperature shown in Table 5 . As the atmosphere at $10 \mathrm{GHz}$ may change by more than $50 \mathrm{mK}$ over several hours, we do not attempted to calculate temperatures for those times during which $90 \mathrm{GHz}$ data are not available.

The resultant atmospheric and sky measurements form a self-consistent data set. Figure 7 show the meanured zeaith aky temperature plotted against the corrected atmospheric temperature for 1982-1986. The zenith sky temperature measurements include an atmospheric component, but are much lean sensitive to systematic effects than the atmospheric measurements. Igroring a small ( $<15 \mathrm{mK}$ ) galactic componeat, the data should correlate witb a unity slope and an intercept equal to the CMBR anterns remperature. The data are in statistical agreement with the expected fit, indicating there are no serious undetected syatematic errors.

\section{RESULTS}

As is unually the case in CMBR meanurements, ouf uncertainty in the CMBR temperature is dominated by ayatematic effects. The atmosphere is the largest background signal in the experiment; in every year, the total systematic uncertainty is dominated by the uncertainty in the atmospheric temperature. We beve used three differeat methods to calculate the atmopheric temperature, with correspondingly different aystematic effects. As stated above, we derive an atmonperic temperature for 1982 by correlating resulte at $90 \mathrm{GHz}$ with results at 10 GHz taken in later years. The atmospheric uncertaiaties of 1983 and 1984 are dominated by upper limits to possible systematic angle-dependent changes in offset. Secondary atmospheric measurements are not subject to this effect; the 1986 atmospheric uncertainty is dominated instead by the interference of the primary sereen, an effect not present during primary atmospheric measurements.

Table 6 shows all contributions to the stmospheric systematic uncertainty for a typical year. 1983. The source terms may vary independently; the total uncertainty at each atmospheric angle is thus the quadrature sum of the individual atmospheric terms. The data at each angle are independent of dats at other angles. We weight the atmospheric data at each angle by the 
corresponding uncertainty; the uncertainty in the result is given by the inverse squared sum of the individual uncertainties, as shown in the bottom row of Table 6 . Values for 1984 or 1986 may be found by uning the appropriate values for offset and gain variation from Table 1 and 2.

Table 7 show all systematic effects for the CMBR measurement (zenith sky minus atmospheric and other signals, Equation 2). After the atmospheric contribution, the major components are from the cold load and systematic oftset changes. While uncertainties in the absolute load signal do not correlate with atmospheric uncertainties, systematic offset changes due to changing radiometer ponition may, and must be congidered. Although tests reveal small differences in radiometer performance a the zenith angle varies, the difference each year are not consistent from teat to teat. We take the largeat difference each year a upper limits to the magnitude of any angle-dependent effect (Table 1). The effect at any angle in not correlated with the effect at any othez angle; in particular, the up-down offet change in not observed to correlate with the zenith- $\theta$ atmonpheric offert cbange. Accordingly, we add the limite on offect change in quadrature with the atmospheric and other systematic effect to derive a total systematic uncertainty on the CMBR temperature.

We find the CMBR antenns temperature $T_{A, C M B R}$ by evaluating the terms in Equation 2. shown in Table 8 a averages over each run. The most important terms are the zenith sky - cold load temperature difference $G \Delta V$ (column 4) and the atmospheric antenna temperature $T_{A, \text { ntm }}$ (column 6). These data are consistent and correlate as expected. To obtain the beat estimate of the CMBR temperature, we solve Equation 2 on a scan-by-scan basis for each year and fit the resultant CMBR dats to a Gausuian. The resultant CMBR temperatures for all four years are shown in Table 9. The atatiotical scatter of the CMBR data after the atmosphere and other sigmalo bave been removed is given in column 4; the total uncertainty is the quadrature sum of the statistical uncertainly and the systematic uncertainties of Table 7.

The CMBR data from 1982 to 1984 were well deacribed by Gaussian distributions. In 1986, we obtained only 26 data points, which showed a much larger R.MS than expected. Each data point wa the average of one scan, and had an asociated uncertainty consistent with that expected 
from radiometer noise. From one scan to the next, however, there were large $(\sim 100 \mathrm{mK})$ jumps which could not be explained by radiometer noise alone. We conjecture that the jumps were caused by equipment shocks induced by rapid movement of the radiometer between pusitions while taking dats at White Mountain, causing a random shift in the offet. Shocks would be expected to generate random oftset changes of either sign, independent of angle, whose average over many repetitions in zero. This is supported by the observed bebaviour of the effect: the output changed relative to the previous sean only at the $180^{\circ}$ and $0^{\circ}$ positions, for which the largest movement and shocks were generated. No effect was observed in offet teats, which involved much gentler movemente of the inotrumeat and teat for repeatable non-random aystematic ehangen. Integration teats with both horn viewing the reaith taken between two runs over the cold load showed no effect, ruling out mont intermittent surces of interfereace whose sigisl rould not be expected to average te zero (ground loopn, interference with other radiometers, radio-frequency interference). We concluded that the effect served to injeet noise to the output signal at $0^{\circ}$ and $180^{\circ}$, but did not alter the mean of the sigaal difference between the two angles when averaged over many scans. With this additional source of noise, the RMS noise of the data integrated down to the $47 \mathrm{mK}$ level. We took the value of $47 \mathrm{mK}$ a a $68 \%$ confidence level for the extimate of the uncertainty of the mean of the pareat distribution.

The final 1986 uncertainty of $83 \mathrm{mK}$ was still dominated by the systematic uncertainty in the atmospheric measuremeat. In the unlikely event that the effect causing the increased R.MS also changed the mean, the moat conservative estimate of the uncertainty is $180 \mathrm{mK}$, balf the total spread in the 1986 data. As the 1986 results were in agreement with the results of previous years, there is no statistically gignificant evidence to justify such a procedure.

Column 5 of Table 9 liste the thermodynamic temperature of the CMBR as determined each year, along with the total extimated uncertainty. The uncertainty is dominated in each year by different systematic effects. The $90 \mathrm{GHz}$ correlation uncertainty, any angle-dependent offset changes, and the primary screen interference are all independent effects. The results for all years are in agreement with each other. Since mont of the total uncertainty is systematic, not statistical, 
and wince the oyotematic uncertainty is largely independent each year, we have averaged the values in Table 9 weighted by their total atimated uncertainty. The resultant CMBR thermodynamic temperature of $10 \mathrm{GHz}_{2}$ is

$$
T_{\text {A.CMBR }}=2.61 \pm 0.06 \mathrm{~K} \text {. }
$$

The combined result difen from previously publinhed eatimates of the CMBR temperature using the same radiometer (Smoot et al. 1985a,b) by more than the atimated error. This is largely due to reanalysis of the 1982 atmospheric temperature made possible by three years of additional atmoupheric data at 10 and $90 \mathrm{GHz}$. The smallez uncertainty is largely due to the use of the mecondary atmoepheric scans in 1986, which bave greatly improved our ability to monitor the temperature of the atmosphere.

\section{COMPARISON WITH RELATED MEASUREMENTS}

Prior to the start of our effort, the only CMBK messurements near $3.0 \mathrm{~cm}$ wavelength were taken at $3.2 \mathrm{~cm}$ shortly arter the discovery of the existence of the CMBR. Roll and Wilkinson (1966) reported $T_{A, C M B R}=3.0 \pm 0.5 \mathrm{~K}$, while Stokes et al. (196i) reported a CMBR temperature of 2.69-0.16. These data are in agreement with our results. Table 10 lists results of recent CMBR opeetral mesourements scros a wider frequency range. Taken together, these data are consistent with an undistorted blackbody with a temperature of $2.741 \pm 0.016 \mathrm{~K}$. The strictest limits on energy-releasing processes in the early universe occur for energy released at a redshift : between $10^{5}$ and $10^{6}$. Compton-ecattering distorts the CMBR speetrum to higher frequencies, while bremetrahlung bas largely filled in the low-frequency deficit with new photons, leaving a narrom colder tranation region in the low-frequency spectrum (Danese and De Zotti 1980). The best fit to such a spectrum yielde a fractional energy release $\Delta E / E_{C . M B R}=0.0015$ with $x^{2}$ of 21.2 for 16 degrees of freedom. Fit to an undintorted apectrum $(\Delta E / E=0)$ by comparison bave $x^{2}=$ 22.5 for 17 DOF. (The coumological baryca density parameter $\Omega_{t}$ bas sen taken to be 0.1 for the purposes of this discussion). A more complete dissussion of the CMBR epectrum and resulting conmological implications will be published in a iorthcoming paper (Smoot et al. 1987b). 


\section{ACKNOWLEDGEMENTS}

We gratefully acknowledge the skilled belp of many people over the course of this experiment:

J. Aymon, B. Dougherty, J. Gibson, N. Gusack, F. Mitschang, and the staff and crew of the White Mountain Research Station. This work was supported by NSF grants PEY 80-15694 and AST 800737, by the Department of Energy under Contract DE-AC03-76SF00098, by C.N.R. Fellowships nos. 203.2.13 and 203.2.15, sad by ISTRA (Milano). 


\section{REFERENCES}

Bielli, P., Pagana, E., and Sironi, G. 1983, Proceedings ICA P, 1, 509.

Davies, R. D., Lasenby, A. N., Watson, R. A., Daintree, E. J., Hopkins, J., Beckman, J., SanchezAlmeida, J., and Rebolo, R. 1987, Nature, 326, 462.

Costales, J. B., Smoot, G. F., Witebsky, C, De Amici, G., and Friedman, S. D. 1986, Radio Science, 21, 47.

Crane, P., Heygi, D. J., Mandolesi, N., and Danke, A. C. 1986, Ap. J., $309,822$.

Danese, L., and De Zotti, G. 1977, Riv. Nuovo Cimento, T, 277.

-. 1978, Astr. Ap., 68, 157.

-. 1980, Astr. Ap., 84, 364.

De Amici, G., Smoot, G. F., Aymon, A., Bersanelli, M., Kogut, A., Levin, S. M., and Witebsky, C. 1987, submitted to Ap. J.

Friedman, S. D. 1984, Ph.D. Theais, Univ. of Ca. Berkeley, LBL \#17279.

Batam, C. G. T., Salter, C. J., Stoffel, B., and Wileon, W. E. 1982 Astr. Astroph. Suppl. Ser., 47, 1 .

Johneon, D. G., and Wilkineon, D. T. 1986, Ap. J. Lett, 320, LL-L3.

Kraw, J.D. 1966, Radio Astronomy (New York: McGram-Bill).

Mardolesi, N., Calzolari, P., Cortiglioni, S., Morigi, G. 1986, Ap. J., 310, 561.

Meyer, D. M., and Jura, M. 1985, Ap. J., $297,119$. 
Partridge, R. B., Cannon, J., Foster, R., Johnson, C., Rubinstein, E., Rudolph, A., Danese, L., and De Zotti, G. 1984, Phys. Rev. D, 29, 2683.

Peterson, J. B., Richards, P. L., and Timusk, T. 1985, Phys. Rev. Lett., 55, 332.

Roll, P. G., and Wilkinson, D. T. 1966, Phys. Rev. Lett., 16, 405.

Sironi, G., and Bonelli, G. 1987, A p. J., in press.

Smoot, G.F., et al 1983, Phys. Rev. Lett., 31, 1009.

-. 1985a, Ap. J. Lett.,

Smoot, G. F., De Amici, G., Levin, S., and Witebsky, C. 1985b, Società Ilaliana di Fisica Proceedings 1, 27.

Smoot, G. F., Bensadoun, M., Bersanelli, M., De Amici, G., Kogut, A., Levin, S., and Witebsky, C. 1987 a, Ap. J. Lett., 317, L345.

Smoot, G. F., De Amici, G., Levin, S., and Witebsky, C. 198it, in preparation.

Stokes, R. A., Partridge, R. B., and Wilkineon, D. T. 1967, Phys. Rev. Lett., 19, 1199.

Sunyaev, R. A., and Zel'dovich, Y. B. 1970, Ap. Space Ser., 7, 20.

Wilebsky, C., Smoot., G. F., De Amici, G., and Friedman, S. D. 1986, Ap. J., 310, 145. 


\section{FIGURE CAPTIONS}

Figure 1. Atmospheric and peak galactic emission for a site at $3800 \mathrm{~m}$ elevation. The atmospheric emission is dominated by oxygen $\left(O_{2}\right)$ and includes the contribution from typical Water vapor column density of $0.25 \mathrm{~g} \mathrm{~cm}^{-2}$.

Figure 2. Liquid-belium-cooled target used as a reference load.

Figure 3. Scbematic of the radiometer.

Figure 4. Radiometer configuration in 1982 . The 1983 configuration is similar with slightly larger sereens.

Figure 5. Radiometer configuration in 1986.

Figure 6a. Messured $10 \mathrm{GEz}$ va $90 \mathrm{GHz}$ atmoapheric antenna tempetature. Solid line is best fit to dass exeluding the 1982 reaults.

Figure $6 \mathrm{~b} .10 \mathrm{GHz}$ zenith sky ve $90 \mathrm{GHz}$ atmospheric antenna temp.srature. Solid line is best fit to all data.

Figure 7. Zenith sky vs calculated atmospheric antenna temperature. Solid line is the expected fit for $T_{A, C M B R}=2.38 \mathrm{~K}$. 
M. Bersanelli, G. De Amici, A. Kogut, S. Levin, G. Smoot, and C. Witebsky: 50/232 Lawrence Berkeley Laboratory, University of Californiz, Berkeley, CA 94720

S. D. Friedman: CASS C-011, University of California at San Diego, La Jolla, CA 92093

M. Griffith and B. Grosuan: 37/624D Center for Space Research, Massachusetts Institute of Technology, Cambridge, MA 02139 
TABLE 1

Upper limits to Systematic Offset Changes *

\begin{tabular}{ccc}
\hline $\begin{array}{c}\text { Year } \\
\text { Tested }\end{array}$ & $\begin{array}{c}\text { Warm Target } \\
\text { (mKi }\end{array}$ & $\begin{array}{c}\text { Cold Target } \\
\text { (mK) }\end{array}$ \\
\hline 1982 & $15 \pm 4$ & $40 \pm 8$ \\
1983 & $41 \pm 8$ & $43 \pm 8$ \\
1984 & $65 \pm 15$ & $0 \pm 600^{\mathrm{b}}$ \\
1986 & $24 \pm 6$ & $6 \pm 17$ \\
\hline
\end{tabular}

- Warm targets are rensitive to gain and offset changea.

Cold targets are sensitive only to offset changes.

b No precise cold measurements were made in 1984.

TABLE 2

Measured Gain Saturation

\begin{tabular}{lc}
\hline \hline Year & Saturation (\%) \\
\hline 1982 & $6 \pm 1$ \\
1983 & $1.5 \pm 1.0$ \\
1984 & $1.8 \pm 0.2$ \\
1986 & $1.8 \pm 0.1$ \\
\hline
\end{tabular}


TABLE 3

Measured atmospheric antenna Temperuture *

\begin{tabular}{|c|c|c|c|c|c|c|c|}
\hline Date & $\begin{array}{l}\text { Time } \\
\text { (UT) }\end{array}$ & $\begin{array}{c}\text { Gain } \\
(\mathrm{mK} / \mathrm{d} u)\end{array}$ & $\begin{array}{l}T 30 \\
(K)\end{array}$ & $\begin{array}{l}\mathrm{T} 40 \\
(\mathrm{~K})\end{array}$ & $\begin{array}{l}\mathrm{T} 47 \\
(\mathrm{~K})\end{array}$ & $\begin{array}{l}\text { T54 } \\
\text { (K) }\end{array}$ & $\begin{array}{c}T_{\text {A, atm }} \\
(\mathrm{K})\end{array}$ \\
\hline \multicolumn{8}{|c|}{1982 Primary Atmouphere ${ }^{b}$} \\
\hline 5 July & $\begin{array}{l}: 08-5: 48 \\
12: 00-12: 30 \\
3: 09-3: 54 \\
6: 25-7: 22 \\
11: 45-12: 22 \\
\end{array}$ & $\begin{array}{l}9.71 \pm 0.10 \\
9.65 \pm 0.10 \\
9.75 \pm 0.10 \\
9.69 \pm 0.10 \\
9.68 \pm 0.10\end{array}$ & $\begin{array}{l}1.00 \pm 0.16 \\
0.91 \pm 0.16 \\
0.99 \pm 0.16 \\
0.90 \pm 0.16 \\
0.91 \pm 0.16\end{array}$ & $\begin{array}{l}\ldots \\
\ldots \\
\ldots \\
\ldots \\
\ldots \\
\end{array}$ & $\begin{array}{l}\ldots \\
\cdots \\
\cdots \\
\cdots \\
\cdots\end{array}$ & $\begin{array}{l}\cdots \\
\cdots \\
\cdots \\
\cdots \\
\cdots \\
\end{array}$ & $\begin{array}{l}1.00 \pm 0.16 \\
0.91 \pm 0.16 \\
0.99 \pm 0.16 \\
0.90 \pm 0.16 \\
0.91 \pm 0.16\end{array}$ \\
\hline \multicolumn{8}{|c|}{1983 Primary Atmosphere } \\
\hline $\begin{array}{l}4 \text { Sept } \\
5 \text { Sept }\end{array}$ & $\begin{array}{l}8: 26-9: 18 \\
3: 32-4: 17 \\
9: 01-9: 38 \\
7: 03-8: 17\end{array}$ & $\begin{array}{l}8.95 \pm 0.10 \\
8.95 \pm 0.10 \\
8.91 \pm 0.10 \\
8.87 \pm 0.10\end{array}$ & $\begin{array}{l}1.02 \pm 0.28 \\
1.05 \pm 0.28 \\
1.06 \pm 0.28 \\
1.37 \pm 0.28\end{array}$ & $\begin{array}{l}1.21 \pm 0.14 \\
1.20 \pm 0.14 \\
1.19 \pm 0.14 \\
1.22 \pm 0.14\end{array}$ & $\begin{array}{l}\cdots \\
\cdots \\
\cdots \\
\cdots\end{array}$ & $\begin{array}{l}\cdots \\
\cdots \\
\cdots \\
\cdots\end{array}$ & $\begin{array}{l}1.17 \pm 0.13 \\
1.17 \pm 0.13 \\
1.16 \pm 0.13 \\
1.25=? .13\end{array}$ \\
\hline \multicolumn{8}{|c|}{1984 Primary Atmosphere } \\
\hline $\begin{array}{l}24 \text { Aug } \\
25 \text { Aug }\end{array}$ & $\begin{array}{l}10: 36-11: 27 \\
12: 32-13: 17 \\
7: 04-7: 51\end{array}$ & $\begin{array}{l}8.86 \pm 0.03 \\
8.85 \pm 0.03 \\
8.67 \pm 0.03\end{array}$ & $\begin{array}{l}1.03 \pm 0.43 \\
1.00 \pm 0.43 \\
1.18 \pm 0.43\end{array}$ & $\begin{array}{l}1.09 \pm 0.22 \\
1.10 \pm 0.23 \\
1.16 \pm 0.22\end{array}$ & $\begin{array}{l}\cdots \\
\ldots \\
\ldots\end{array}$ & $\begin{array}{l}\cdots \\
\cdots \\
\ldots\end{array}$ & $\begin{array}{l}1.07 \pm 0.20 \\
1.08 \pm 0.20 \\
1.17 \pm 0.20\end{array}$ \\
\hline \multicolumn{8}{|c|}{1986 Secondary Atmoupbere } \\
\hline $\begin{array}{l}8 \text { Aug } \\
9 \text { Aug }\end{array}$ & $\begin{array}{l}2: 53-3: 34 \\
10: 12-11: 38 \\
3: 11-4: 18 \\
9: 14-10: 06\end{array}$ & $\begin{array}{l}9.24 \pm 0.03 \\
9.13 \pm 0.03 \\
9.49 \pm 0.03 \\
9.17 \pm 0.03\end{array}$ & $\begin{array}{l}1.21 \pm 0.09 \\
1.12 \pm 0.09 \\
1.29 \pm 0.09 \\
1.12 \pm 0.09\end{array}$ & $\begin{array}{l}1.22 \pm 0.05 \\
1.16 \pm 0.05 \\
1.27 \pm 0.05 \\
1.17 \pm 0.05\end{array}$ & $\begin{array}{l}1.26 \pm 0.03 \\
1.18 \pm 0.03 \\
1.30 \pm 0.03 \\
1.20 \pm 0.03\end{array}$ & $\begin{array}{l}1.27 \pm 0.02 \\
1.22 \pm 0.02 \\
1.32 \pm 0.02 \\
1.22 \pm 0.02\end{array}$ & $\begin{array}{l}1.25 \pm 0.07 \\
1.17 \pm 0.07 \\
1.30 \pm 0.07 \\
1.18 \pm 0.07\end{array}$ \\
\hline
\end{tabular}

- Quantitie T30, T40, T47, and T54 (column 4-7) are the mesa atmoopheric anteona cemperatures for $\pm 30^{\circ}, \pm 40^{\circ}, \pm 47^{\circ}$, and $\pm 54^{\circ}$, respectively. $T_{A, a t m}$ is the appropriately weigbled mean value (cee text). A du in a digiitized unit of radiometer output. Errors quoted are $68 \%$ confidence level limits.

b The uncertainty for $T_{A \text {.akm }}$ for 1982 may be an underestimate (see lext).

c Prior to 1986, only primary stmospheric measuremente were made. For 1986, only the more accurate secondary results are abown. 
TABLE 4

Comparison of 1986 Primary and Secondary atmospherjc Measurements

\begin{tabular}{llccc}
\hline $\begin{array}{l}\text { Date } \\
(1986)\end{array}$ & $\begin{array}{l}\text { Time } \\
\text { (UT) }\end{array}$ & $\begin{array}{c}\text { Linear Drift } \\
(\mathrm{mK} / \text { hour })\end{array}$ & $\begin{array}{c}\text { Primary } T_{\text {A,cm }} \\
(\mathrm{K})\end{array}$ & $\begin{array}{c}\text { Secondary } T_{A, \mathrm{~mm}} \\
(\mathrm{~K})\end{array}$ \\
\hline 8 Aug & $4: 40-5: 27$ & $-11 \pm 1$ & $1.24 \pm 0.12$ & $1.23 \pm 0.07$ \\
& $7: 48-8: 24$ & $\ldots$ & $1.19 \pm 0.12$ & $1.20 \pm 0.07$ \\
9 Aug & $5: 51-6: 21$ & $-23 \pm 7$ & $1.17 \pm 0.12$ & $1.24 \pm 0.07$ \\
& $7: 35-8: 11$ & $\ldots$ & $1.15 \pm 0.12$ & $1.20 \pm 0.07$ \\
\hline
\end{tabular}

- The quoted erron are $68 \%$ confidence level limits and include systematic uncertainties.

TABLE 5

Calculated atmojpheric antenna temperature *

\begin{tabular}{llccc}
\hline \hline $\begin{array}{l}\text { Date } \\
\text { (1982) }\end{array}$ & $\begin{array}{l}\text { Time } \\
\text { (UT) }\end{array}$ & $\begin{array}{c}\text { Mesaured } 90 \mathrm{GHz} \\
T_{\text {A.um }}(\mathrm{K})^{\mathrm{b}}\end{array}$ & $\begin{array}{c}\text { Measured } 10 \mathrm{GHz} \\
T_{\text {A,atm }}(\mathrm{K})\end{array}$ & $\begin{array}{c}\text { Calculated } 10 \mathrm{GHz} \\
T_{\text {A.acm }}(\mathrm{K})\end{array}$ \\
\hline 5 July & $\mathbf{5 : 0 8 - 5 : 4 8}$ & $13.38 \pm 0.57$ & $1.00 \pm 0.16$ & $1.19 \pm 0.11$ \\
& $12: 00-12: 30$ & $\ldots$ & $0.91 \pm 0.16$ & $\ldots$ \\
6 July & $3: 09-3: 54$ & $14.08 \pm 0.57$ & $0.99 \pm 0.16$ & $1.20 \pm 0.11$ \\
& $9: 01-9: 38$ & $13.34 \pm 0.57$ & $0.90 \pm 0.16$ & $1.19 \pm 0.11$ \\
& $11: 45-12: 22$ & $\ldots$ & $0.91 \pm 0.16$ & $\ldots$ \\
\hline
\end{tabular}

- Quoted uncertainties are $68 \%$ confidence level limits. The "Measured $10 \mathrm{GBz}$ " uncertainty (column 4) may be an underestimate (see text).

b Simultaneous $90 \mathrm{GHz}$ values are not available for some observing times. 
TABLE 6

Contributions to ATMOSPheric Systematic ERror *

\begin{tabular}{|c|c|c|c|c|}
\hline Source Term & $\begin{array}{l}\text { Approximate Source } \\
\text { Magnitude }\end{array}$ & $\begin{array}{l}\text { Approximate Source } \\
\text { Uncertainty }\end{array}$ & $\begin{array}{l}\text { Atmospheric } \mathrm{E} \\
30^{\circ}\end{array}$ & $\begin{array}{l}\text { Error }(\mathrm{mK}) \\
40^{\circ}\end{array}$ \\
\hline $\begin{array}{l}\text { Offet Changes } \\
\text { Pointing Repestability } \\
\text { Gain } \\
\text { Sidelobes } \\
\text { Galaxy } \\
\text { Atm Model }\end{array}$ & $\begin{array}{c}0 \mathrm{mK} \\
\sigma \\
9 \mathrm{mK} / \mathrm{du} \\
5 \mathrm{mK} \\
25 \mathrm{mK} \\
\ldots\end{array}$ & $\begin{array}{c}43 \mathrm{mK} \\
4^{\prime} \\
0.10 \mathrm{mK} / \mathrm{du} \\
3 \mathrm{mK} \\
12 \mathrm{mK} \\
3 \%\end{array}$ & $\begin{array}{l}269 \\
10 \\
12 \\
19 \\
39 \\
38\end{array}$ & $\begin{array}{c}135 \\
6 \\
12 \\
10 \\
19 \\
36\end{array}$ \\
\hline $\begin{array}{l}\text { Total } \\
\text { Weighted Total }\end{array}$ & & & 275 & 142 \\
\hline
\end{tabular}

- The value shown are for 1983. Similar values bold for 1984, with larger offet uncertainty. The 1986 error has no oftset term but has an additional obmerved $63 \mathrm{mK}$ effect.

TABLE T

Contributions to CMBR Systematic Error

\begin{tabular}{lccccc}
\hline \hline Source Term & Approximate & \multicolumn{5}{c}{ CMBR Error (mK) } \\
& Value & 1982 & 1983 & 1984 & 1986 \\
\hline Atmosphere & $1.15 \mathrm{~K}$ & 110 & 130 & 200 & 65 \\
Ofiset Change & $0 \mathrm{mK}$ & 40 & 43 & 65 & 17 \\
Gain & $9 \mathrm{mK} / \mathrm{du}$ & $<1$ & $<1$ & $<1$ & $<1$ \\
Cold Load & $3.562 \mathrm{~K}$ & 13 & 13 & 13 & 13 \\
Ground & $0 \mathrm{mK}$ & 3 & 3 & 3 & 3 \\
Galary & $4 \mathrm{mK}$ & 3 & 2 & 2 & 4 \\
& & & & & \\
Total & & 118 & 138 & 211 & 69 \\
\hline
\end{tabular}


TABLE 8

Terms Used to Calculate CMBR antenna Temperature *

\begin{tabular}{|c|c|c|c|c|c|c|c|c|}
\hline \multicolumn{2}{|c|}{ Date } & \multirow{2}{*}{$\begin{array}{l}\begin{array}{l}\text { Time } \\
\text { (UT) }\end{array} \\
\text { 5:08-5:48 } \\
3: 09-3: 54 \\
6: 25-7: 22\end{array}$} & \multirow{2}{*}{$\begin{array}{c}\begin{array}{c}\text { Number } \\
\text { Of Seans }\end{array} \\
15 \\
18 \\
22\end{array}$} & \multirow{2}{*}{$\begin{array}{l}\begin{array}{l}G \Delta V \\
(\mathrm{mK})\end{array} \\
48 \pm 41 \\
72 \pm 41 \\
65 \pm 40\end{array}$} & \multirow{2}{*}{$\frac{\begin{array}{c}T_{\text {A,lond }} \\
(\mathrm{mK})\end{array}}{3562 \pm 13}$} & \multirow{2}{*}{$\begin{array}{c}\begin{array}{c}T_{\text {A. alm }} \\
(\mathbf{m K})\end{array} \\
1189 \pm 110 \\
1195 \pm 110 \\
1185 \pm 110\end{array}$} & \multirow{2}{*}{$\frac{\begin{array}{c}T_{A, \text { ground }} \\
(\mathrm{mK})\end{array}}{0 \pm 3}$} & \multirow{2}{*}{$\begin{array}{c}\begin{array}{c}T_{\text {A.gdexy }} \text { (mK) } \\
\text { (mK) }\end{array} \\
3 \pm 3 \\
3 \pm 3 \\
3 \pm 3\end{array}$} \\
\hline 1982 & $\begin{array}{l}5 \text { July } \\
6 \text { July }\end{array}$ & & & & & & & \\
\hline 1983 & $\begin{array}{l}4 \text { Sept } \\
5 \text { Sept } \\
6 \text { Sept }\end{array}$ & $\begin{array}{l}8: 26-9: 18 \\
3: 32-4: 17 \\
9: 01-: 38 \\
7: 03-8: 17\end{array}$ & $\begin{array}{c}15 \\
13 \\
9 \\
23\end{array}$ & $\begin{aligned}-24 & \pm 43 \\
46 & \pm 44 \\
32 & \pm 44 \\
106 & \pm 45\end{aligned}$ & $3562 \pm 13$ & $\begin{array}{l}1170 \pm 130 \\
1165 \pm 130 \\
1162 \pm 130 \\
1253 \pm 130\end{array}$ & $0 \pm 3$ & $\begin{array}{l}3 \pm 3 \\
5 \pm 3 \\
3 \pm 3 \\
4 \pm 3\end{array}$ \\
\hline 1984 & $\begin{array}{l}24 \text { Aug } \\
25 \text { Aug }\end{array}$ & $\begin{array}{l}10: 36-11: 27 \\
12: 32-13: 17 \\
7: 04-7: 51\end{array}$ & $\begin{array}{c}13 \\
9 \\
12\end{array}$ & $\begin{array}{r}-53 \pm 65 \\
-94 \pm 67 \\
34 \pm 65\end{array}$ & $3562 \pm 13$ & $\begin{array}{l}1074 \pm 195 \\
1075 \pm 205 \\
1166 \pm 195\end{array}$ & $0 \pm 3$ & $\begin{array}{l}1 \pm 2 \\
3 \pm 2 \\
8 \pm 4\end{array}$ \\
\hline 1986 & $\begin{array}{l}8 \text { Aug } \\
9 \text { Aug }\end{array}$ & $\begin{array}{l}7: 48-8: 24 \\
3: 51-8: 21 \\
7: 35-8: 11\end{array}$ & $\begin{array}{l}9 \\
8 \\
9\end{array}$ & $\begin{array}{r}8 \pm 37 \\
40 \pm 35 \\
-68 \pm 33\end{array}$ & $3568 \pm 13$ & $\begin{array}{l}1197 \pm 65 \\
1240 \pm 65 \\
1201 \pm 65\end{array}$ & $0 \pm 3$ & $\begin{array}{c}5 \pm 3 \\
15 \pm 8 \\
7 \pm 4\end{array}$ \\
\hline
\end{tabular}

- The quoted errors are $68 \%$ confidence level limits and include systematic uncertainties.

$G \Delta V$ (column 4) refers to the measured zenith aky - co!: ; load temperature difference.

TABLE 9

CMBR ResulTs FOR $1982-1986^{\bullet}$

\begin{tabular}{lcccc}
\hline \hline Year & $\begin{array}{c}\text { Number of } \\
\text { Observation }\end{array}$ & $\begin{array}{c}T_{\text {A.C.MBR }} \\
(\mathrm{K})\end{array}$ & $\begin{array}{c}\text { Statistical } \\
\text { RMS (K) }\end{array}$ & $\begin{array}{c}\text { CMBR Thermodynamic } \\
\text { Temperature (K) }\end{array}$ \\
\hline 1982 & 55 & 2.430 & 0.035 & $2.66 \pm 0.12$ \\
1983 & 61 & 2.401 & 0.069 & $2.63 \pm 0.14$ \\
1984 & 34 & 2.419 & 0.045 & $2.65 \pm 0.21$ \\
1986 & 26 & 2.331 & $0.093^{b}$ & $2.56 \pm 0.08$ \\
& & & & $2.61 \pm 0.06$ \\
Combined: & & & & \\
\hline
\end{tabular}

- The errors of the CMBR thermodynamic temperature are $68 \%$ confidence level limits and include systematic effects. The combined result is a weighted average of the results of the four years.

b The 1986 data are not well-described by a Gausoian distribution (see text). 
TABLE 10

RECENT MEASUREMENTS OF CMBR THERMOdYNAMIC TEMPERATURE

\begin{tabular}{|c|c|c|c|}
\hline Reference & $\begin{array}{l}\text { Wavelength } \\
\text { (cm) }\end{array}$ & $\begin{array}{l}\text { Frequency } \\
\text { (GEz) }\end{array}$ & $\begin{array}{c}T_{C B R} \\
(\mathbf{K})\end{array}$ \\
\hline Sironi et al. 1987 & 50.0 & 0.6 & $2.45 \pm 0.70$ \\
\hline Levin (Smoot et al. 1987 a) & 21.2 & 1.41 & $2.22 \pm 0.55$ \\
\hline Sironi (Smoot et al. 19858). & 12.0 & 2.5 & $2.78 \pm 0.13$ \\
\hline De Amici (De Amici et al. 1987) & B.1 & 3.7 & $2.58 \pm 0.13$ \\
\hline Mandolesi et al. 1986 & 6.3 & 4.75 & $2.70 \pm 0.07$ \\
\hline Kogut et al. 1987 & 3.0 & 10.0 & $2.61 \pm 0.06$ \\
\hline Johneod and Wilkinson 1986 & 1.2 & 24.8 & $2.783 \pm 0.025$ \\
\hline De Amiei (Smool et al. 19856) & 0.909 & 33.0 & $2.81 \pm 0.12$ \\
\hline $\begin{array}{l}\text { Bersanelli and Witebaky } \\
\text { (Smoot et al. 1987a) }\end{array}$ & 0.333 & 90.0 & $2.60 \pm 0.10$ \\
\hline $\begin{array}{l}\text { Meyer \& Jura } 1985 \\
\text { Meyer \& Jura } 1985\end{array}$ & $\begin{array}{l}0.264 \\
0.132\end{array}$ & $\begin{array}{l}113.6 \\
227.3\end{array}$ & $\begin{array}{l}2.70 \pm 0.04 \\
2.76 \pm 0.20\end{array}$ \\
\hline $\begin{array}{l}\text { Crane et al. } 1986 \\
\text { Crane et ol. } 1986\end{array}$ & $\begin{array}{l}0.264 \\
0.132\end{array}$ & $\begin{array}{l}113.6 \\
227.3\end{array}$ & $\begin{array}{l}2.74 \pm 0.05 \\
2.75_{-0.29}^{+0.24}\end{array}$ \\
\hline Peterson, & 0.351 & 85.5 & $2.30 \pm 0.16$ \\
\hline Richard, \& & 0.198 & 151 & $2.95+0.11$ \\
\hline \multirow[t]{3}{*}{ Timusk 1985} & 0.148 & 203 & $2.92 \pm 0.10$ \\
\hline & 0.114 & 264 & $2.65_{-0.10}^{+0.09}$ \\
\hline & 0.100 & 299 & $2.55_{-0}^{+0} 14$ \\
\hline
\end{tabular}

- This work 


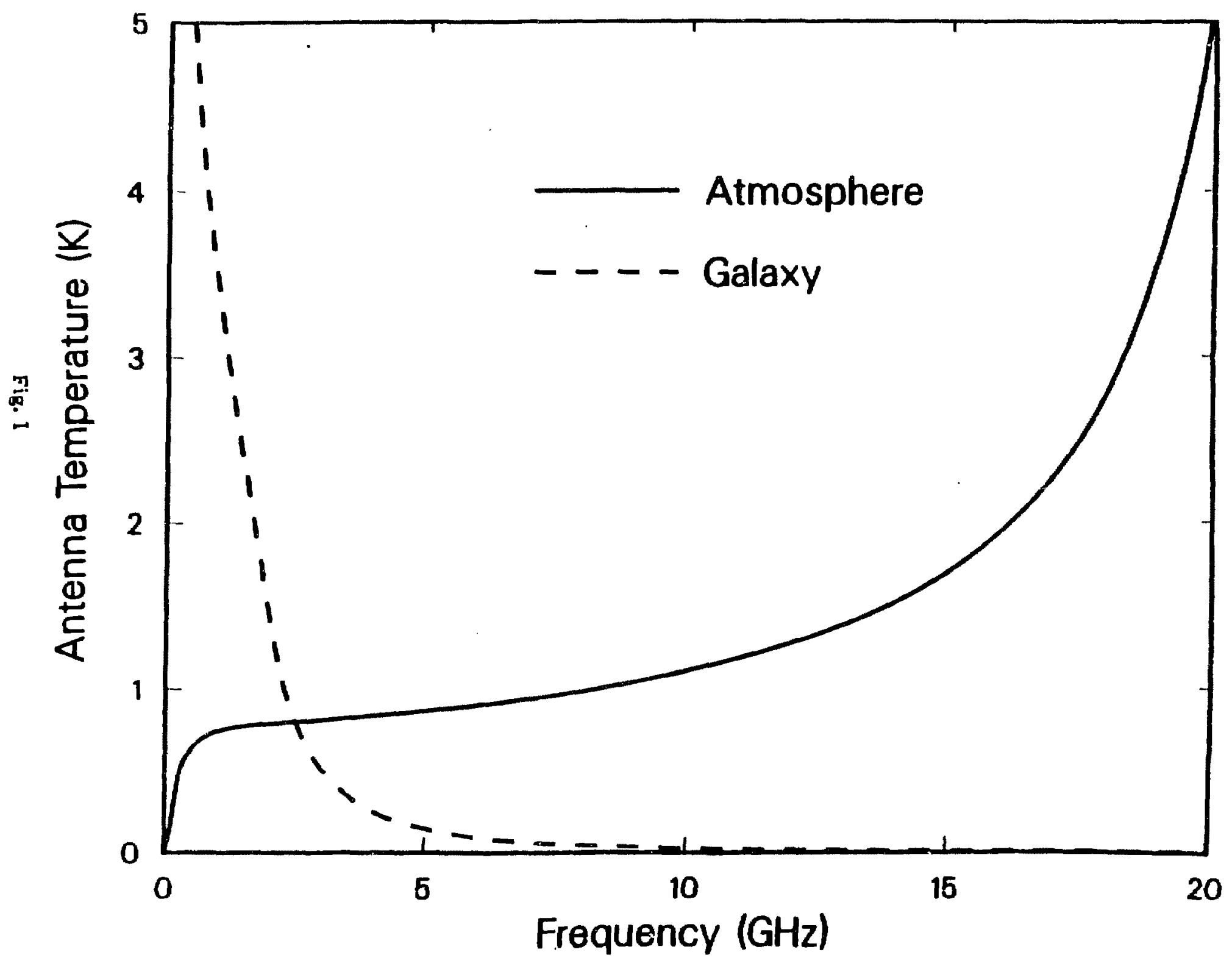




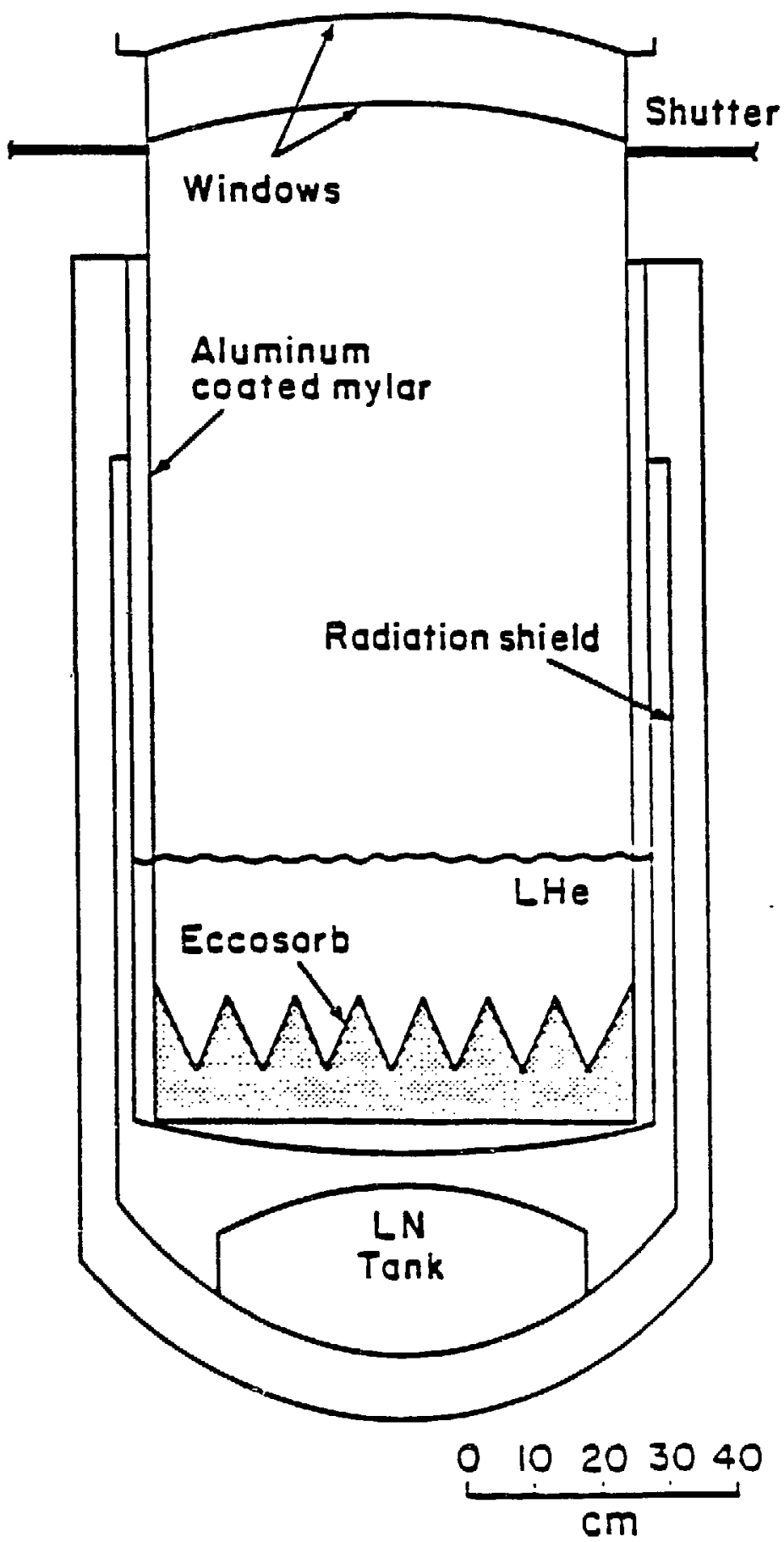

Fig. 2 


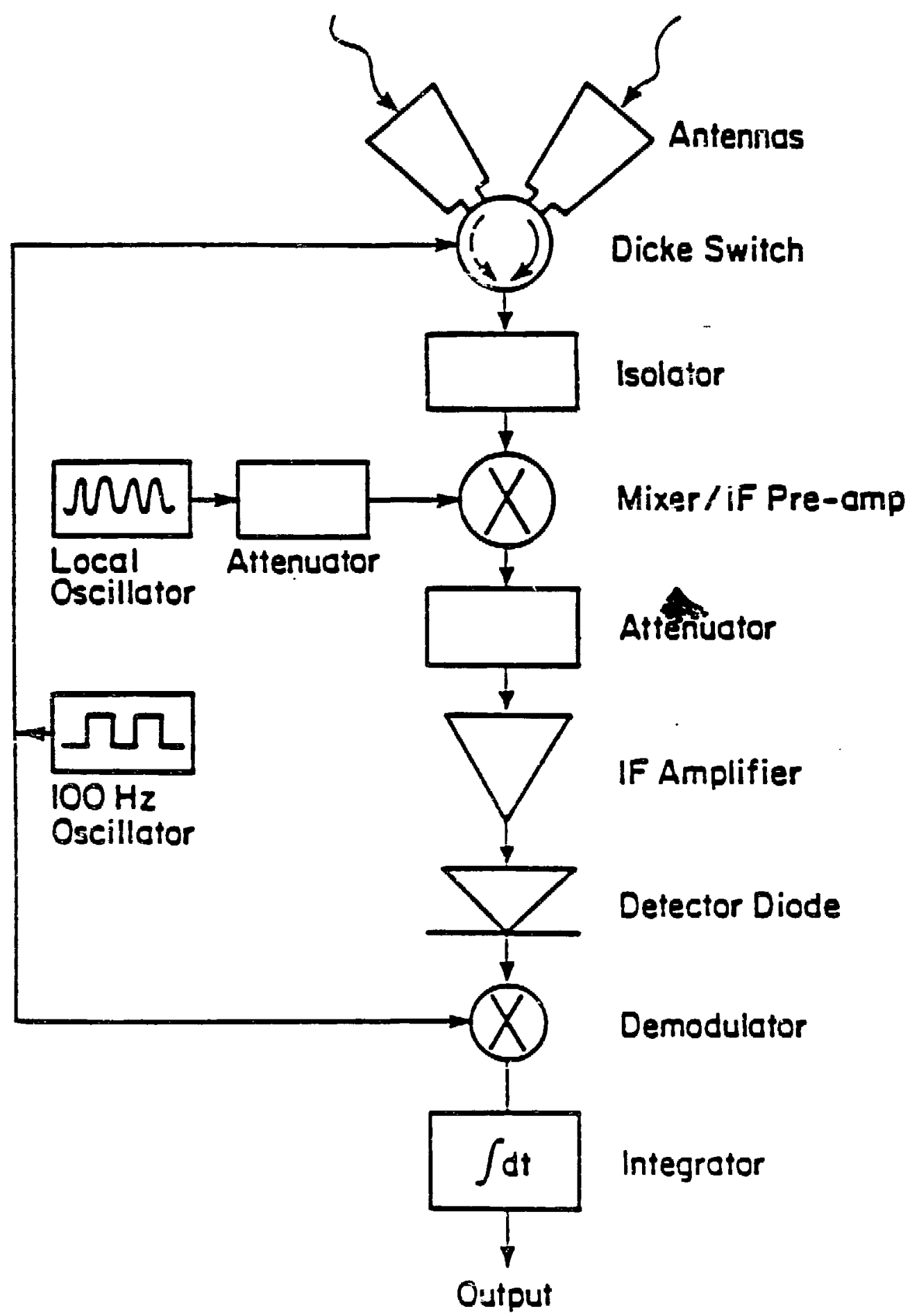

Fig. 3 


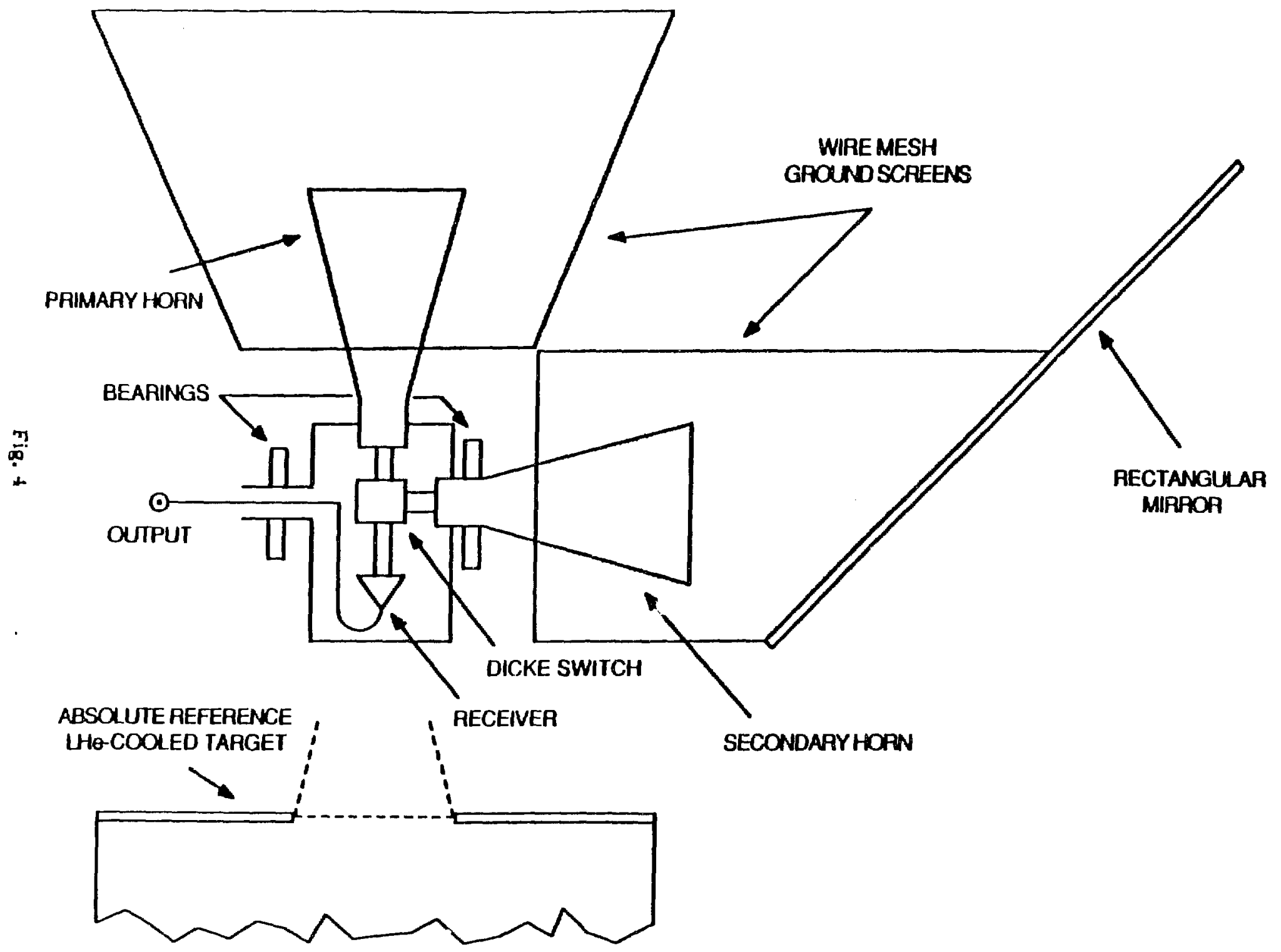




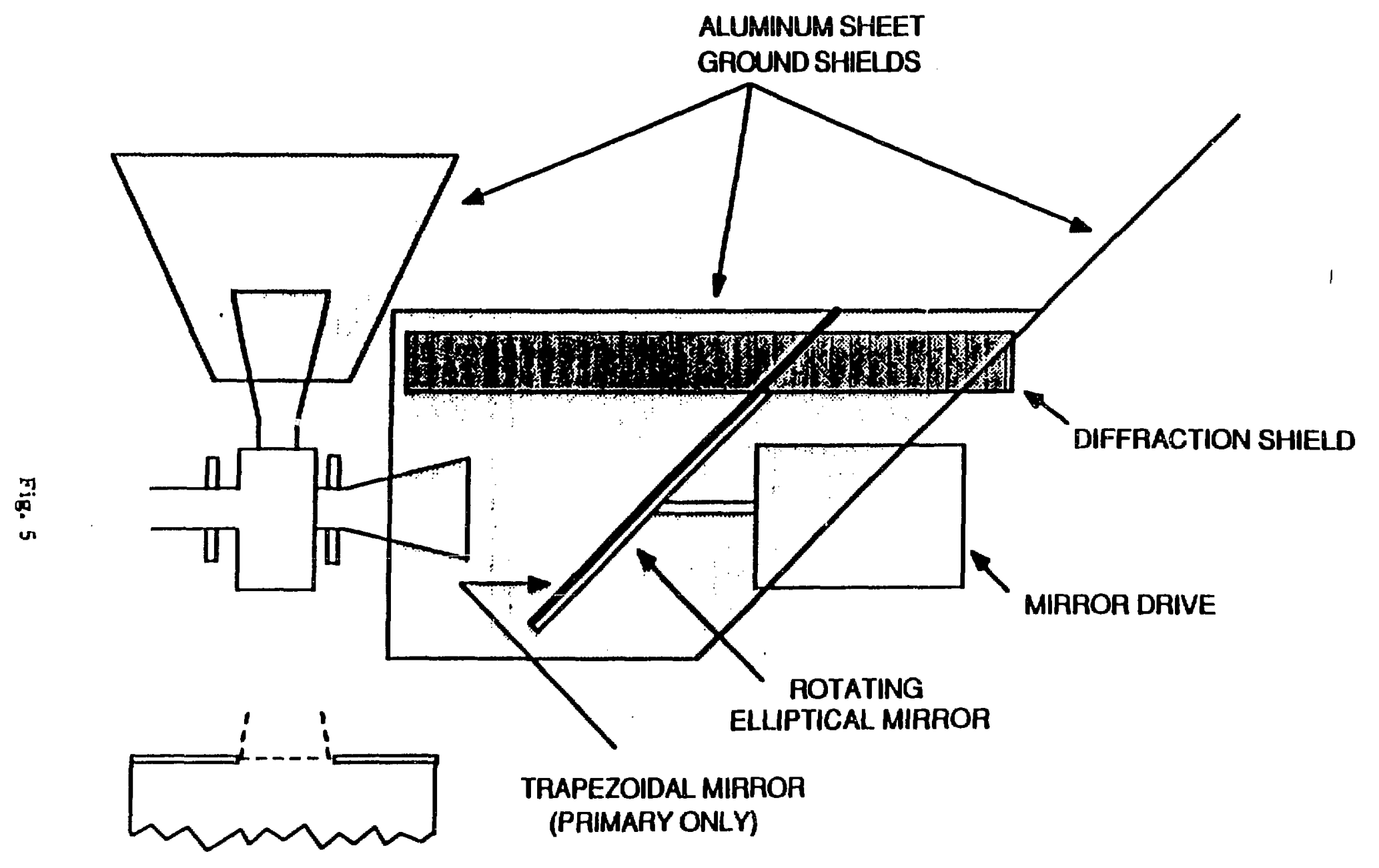

ABSOLUTE REFEPENCE LHe-COOLED TARGET

(PAIMARY ONLY) 


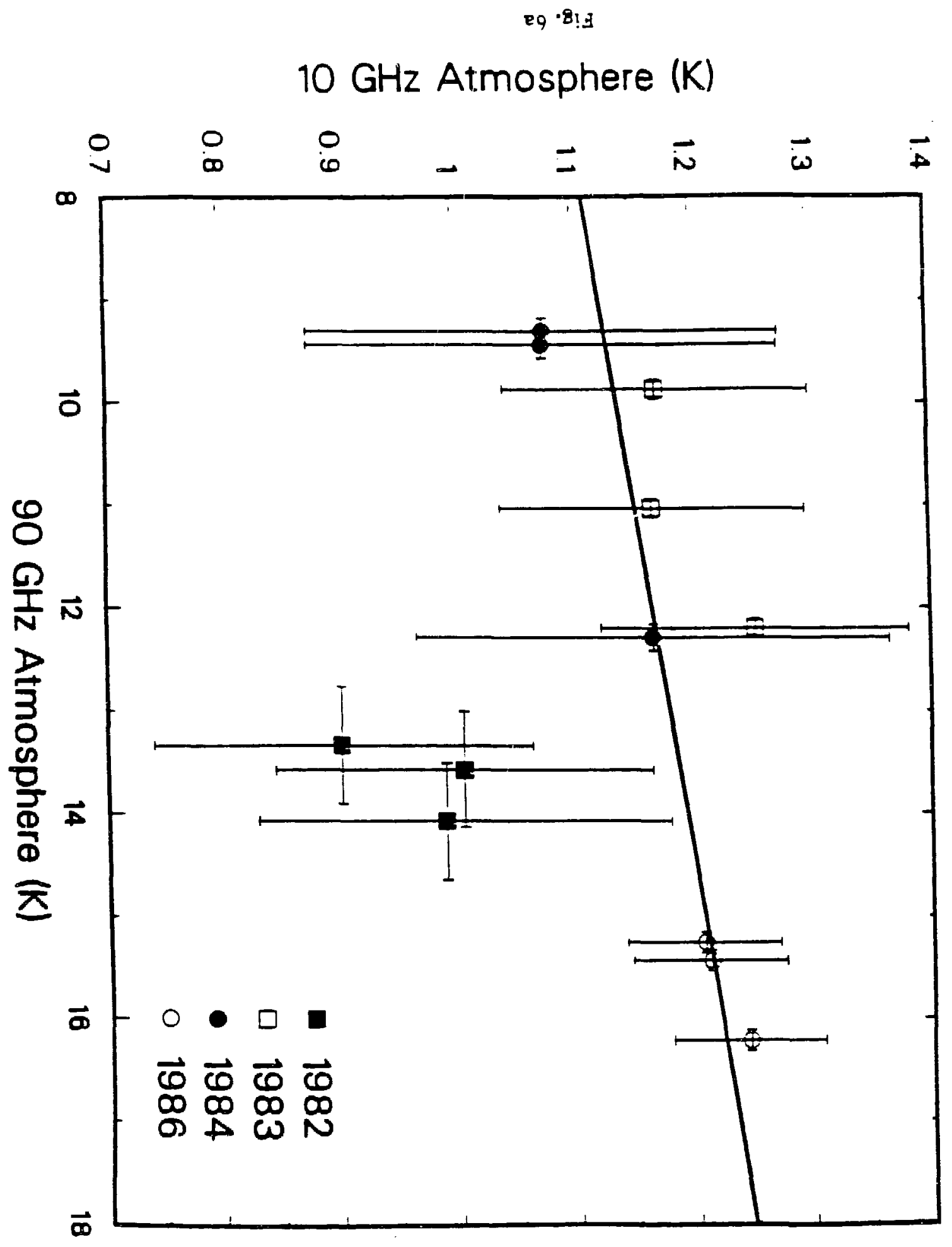




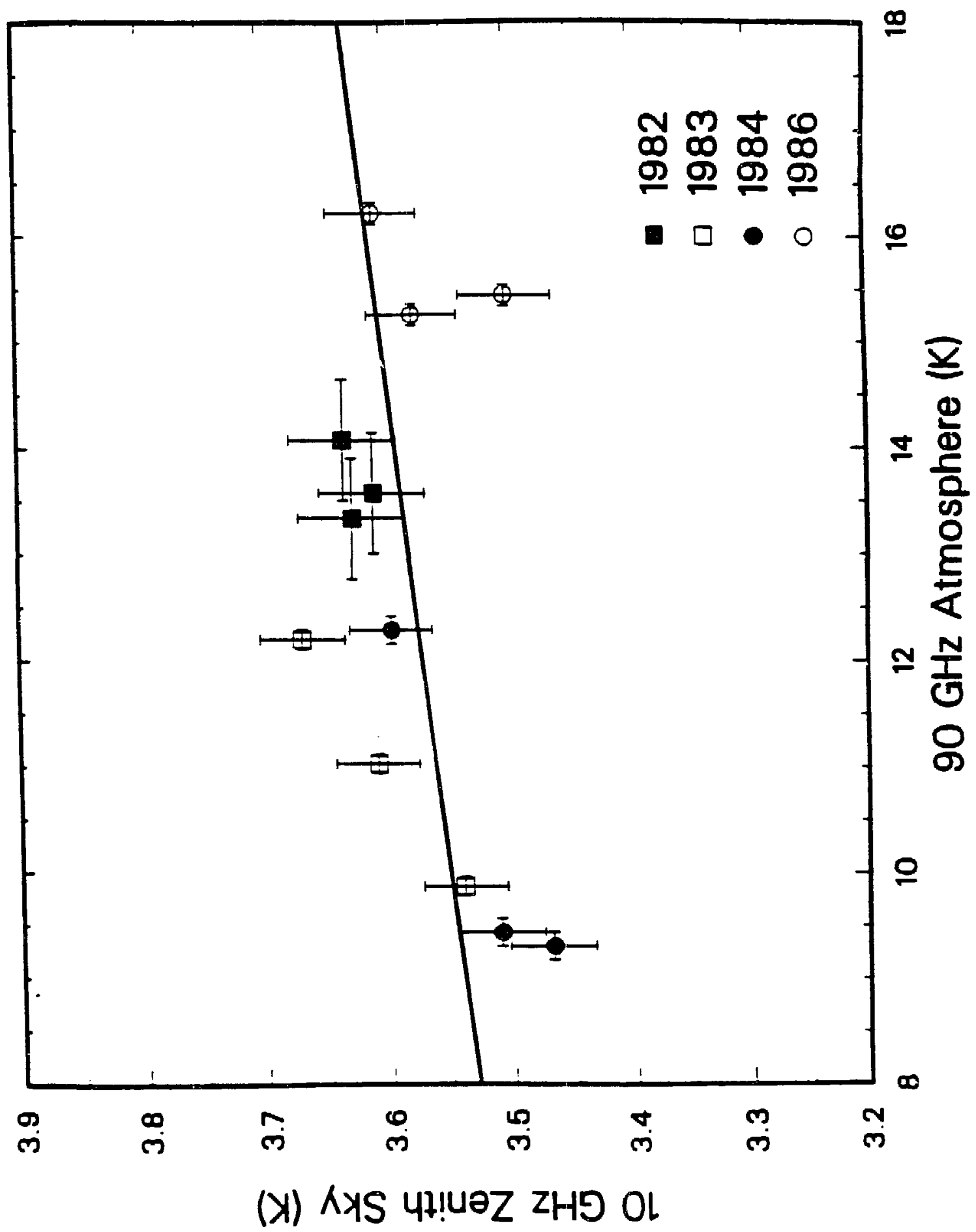

Fig. ob 


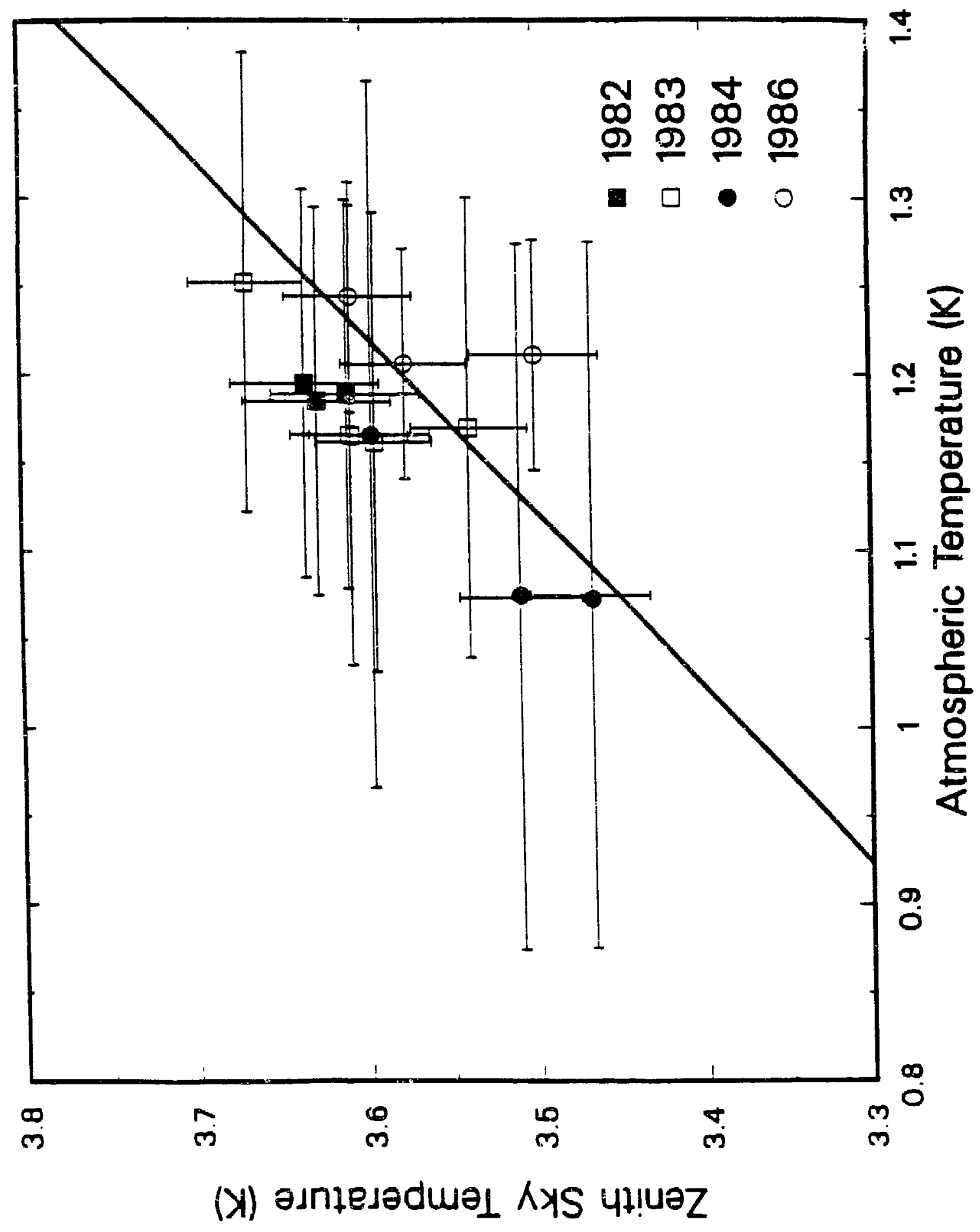

Fig. 7 


\section{Appendix B \\ Antenna Temperature of the Reference Load}

The reference target/radiometer combination may be modelled without loss of generality as a perfect radiometer observing a perfect absorber, with a series of reflecting and absorbing surfaces between them. The antenna temperature of the load is then the sum of the power emitted by the load (attenuared by the surfaces between the load and the radiometer) and the power broadcast by the radiometer and reflected back by the load (in practice, the attenuation is negligible). We will consider these two terms independently.

\section{B.1: Reflection}

The reflection coefficient of the cold load is the coherent sum of the individual amplitude reflection coefficients. Radiation reflecting from surfaces within a coherence length $L$ of the radiometer can interfere coherently with the broadcast radiation. We include this effect as an effective phase term for each reflecting surface, where the phase of reflections internal to the radiometer has been selected as the reference phase. The amplitude reflection coefficient $r$ of the cold load is thus

$$
\begin{aligned}
r=r_{R}+r_{G 1} e^{i \phi G 1}+r_{G 2} e^{i \phi G 2}+r_{H} e^{i \phi H}+r_{A} e^{i \phi A} \\
+r_{P 1} e^{i \phi P 1}+r_{P 2} e^{i \phi P 2}+r_{T} e^{i \phi T}
\end{aligned}
$$

where subscripts $R, G, H, A, P$, and $T$ refer to the radiometer, glass/teflon windows, helium liquid/gas interface, microwave absorber, polyethylene windows, and transition from hom to radiometric cavity, respectively. The magnitudes of the last three terms are small, and may be niglected with negligible error:

$$
r \approx r_{R}+r_{G 1} e^{i \phi G 1}+r_{G 2} e^{i \phi G 2}+r_{H} e^{i \phi H}+r_{A} e^{i \phi A}
$$

The reflected signal is proportional to the square of the reflection coefficient and the difference in broadcast temperature between the radiometer and the cold load,

$$
\Delta \text { Tref }=\mid r^{2}\left(T_{\text {Broad }}-T_{A b s}\right)
$$

where the temperature of the load to lowest order is simply the tempsrature of the absorber. 
Squaring Eq. B2 gives the sum

$$
\begin{aligned}
|r|^{2}=\left|r_{R}\right|^{2} & +\left|r_{G 1}\right|^{2}+\left|r_{G 2}\right|^{2}+\left|r_{H}\right|^{2}+\left|r_{A}\right|^{2} \\
& +2 r_{R} r_{G 1} \cos \left(\phi_{G 1}\right) \\
& +2 r_{R} r_{G 2} \cos \left(\phi_{G 2}\right) \\
& +2 r_{R} r_{H} \cos \left(\phi_{H}\right) \\
& +2 r_{R} r_{A} \cos \left(\phi_{A}\right) \\
& +2 r_{G 1} r_{G 2} \cos \left(\phi_{G 1}-\phi_{G 2}\right) \\
& +2 r_{G 1} r_{H} \cos \left(\phi_{G 1}-\phi_{H}\right) \\
& +2 r_{G 1} r_{A} \cos \left(\phi_{G 1}-\phi_{A}\right) \\
& +2 r_{G 2} r_{H} \cos \left(\phi_{G 2}-\phi_{H}\right) \\
& +2 r_{G 2} r_{A} \cos \left(\phi_{G 2}-\phi_{A}\right) \\
& +2 r_{H} r_{A} \cos \left(\phi_{H}-\phi_{A}\right) .
\end{aligned}
$$

Equation B4 is valid for radiation coherent over regions much larger than the separations between any two surfaces. In practice, the $500-\mathrm{MHz}$ bandwidth $\mathrm{B}$ of the radiometer implies a coherence length $\Lambda=c / B=60 \mathrm{~cm}$, comparable to the separations involved. Over distances $x-\Lambda$, the phase coherence diminishes by an amount $\zeta(z)$, where

$$
\zeta(z)=\left[\frac{\sin (z)}{z}\right]^{2},
$$

where the phase difference $z$ is related to the separation $x$ between two surfaces by

$$
z=\frac{2 \pi x}{\Lambda} \text {. }
$$

Each cosine term in Eq. B4 must be multiplied by the function $\zeta(z)$ applicable for the separation between the reflecting surfaces.

In addition, there is an effect from illumination. The hom antenna occupies $\sim 6 \%$ of the aperture of the load; in addition, the radiometer beam diverges as the distance from the hom mouth increases. Only a fraction $\Gamma$ of the power broadcast from the radiometer and reflecting from a surface actually re-enters the antenna; the rest reflects from the top plate and is absorbed by the microwave absorber. Each term in Eq. B4 must also be multiplied by the illumination $\Gamma$ of the reflecting surface. With these corrections, the reflection coefficient of the reference load may be written as 


$$
\begin{aligned}
\mid r^{2} & =\left|r_{R}\right|^{2} \\
& +\left|r_{G 1}\right|^{2} \Gamma_{G 1}+\left|r_{G 2}\right|^{2} \Gamma_{G 2}+\left|r_{H}\right|^{2} \Gamma_{H}+\left|r_{A}\right|^{2} \Gamma_{A} \\
& +2 r_{R} r_{G 1} \cos \left(\phi_{G 1}\right) \Gamma_{G 1} \zeta\left(z_{G 1}\right) \\
& +2 r_{R} r_{G 2} \cos \left(\phi_{G 2}\right) \Gamma_{G 2} \zeta\left(z_{G 2}\right) \\
& +2 r_{R} \Gamma_{H} \cos \left(\phi_{H}\right) \Gamma_{H} \zeta\left(z_{H}\right) \\
& +2 r_{R} r_{A} \cos \left(\phi_{A}\right) \Gamma_{A} \zeta\left(z_{A}\right) \\
& +2 r_{G 1} r_{G 2} \cos \left(\phi_{G 1}-\phi_{G 2}\right) \Gamma_{G 1} \zeta(z G 1, G 2) \\
& +2 r_{G 1} \Gamma_{H} \cos \left(\phi_{G 1}-\phi_{H}\right) \Gamma_{G 1} \zeta(z G 1, H) \\
& +2 r_{G 1} r_{A} \cos \left(\phi_{G 1}-\phi_{A}\right) \Gamma_{G 1} \zeta\left(z_{G 1, H}\right) \\
& +2 r_{G 2} \Gamma_{H} \cos \left(\phi_{G 2}-\phi_{H}\right) \Gamma_{G 2} \zeta(z G 2, H) \\
& +2 r_{G 2} r_{A} \cos \left(\phi_{G 2}-\phi_{A}\right) \Gamma_{G 2} \zeta\left(z, z_{G 2, H}\right) \\
& +2 r_{H} r_{A} \cos \left(\phi_{H}-\phi_{A}\right) \Gamma_{H} \zeta\left(z_{H}, A\right)
\end{aligned}
$$

All terms contributing to Eq. B7 are listed in Table B1. Equation B7 consists of a constant term (proportional to the summed power reflectivity of each surface and the illuminations), a term dependent on the product of the amplitude coefficients of the radiometer and reflecting surfaces (modulated by the phase $\phi$ between the radiometer and the reference load), and a term dependent on the amplitude reflection coefficients within the load (modulated by their separation). The power reflection intemal to the radiometer, $\left|\Gamma_{R}\right|^{2}$, may be neglected as it cancels in the sky-load comparison (provided $T_{A \text {,zenith }}$ is not greatly different from $T_{A, L o a d}$.

The phase-independent terms sum to $\Sigma\left|r_{i}\right|^{2} \Gamma_{i}=2.0 \times 10^{-5}$. Multiplied by the $298 \mathrm{~K}$ temperature difference between the radiometer's broadcast temperature and the absorber temperature, these terms contribute $6 \pm 3 \mathrm{mK}$ to $T_{A, L a d}$.

Terms dependent on the single phase $\phi i$ between the radiometer and the ith reflecting surface can be calculated knowing the positions of each surface. The terms are small: were all of the terms to add coherently, the reflection dependent on radiometer position would sum to $<1 \mathrm{mK}$. As a precaution, we tested for this effect by sliding the radiometer on a specially-constructed extension of the radiometric wall. We objsived no signal while 
changing the phases $\phi_{i}$ by more than $4 \pi$. Terms dependent on the radiometer's position contribute $0 \pm 1 \mathrm{mK}$ to $\mathrm{T}_{\mathrm{A}, \mathrm{L} \text { oad. }}$

Of the remaining terms, only the first has an appreciable magnitude. The term

$$
2 r_{G 1} r_{G 2} \cos \left(\phi_{G 1}-\phi_{G 2}\right) \Gamma_{G 1} \zeta\left(z_{G 1, G 2}\right)
$$

represents coherent reflection between the two glass/teflon IR-blocking windows. The windows are spaced $5 \mathrm{~cm}$ apart; consequently, the phase term between them has a nominal value -1 . In practice, we cannot be sure that gas pressure does not force the windows closer together. We take this term to be $0 \pm 4 \mathrm{mK}$ instead of the nominal $-4 \mathrm{mK}$. Including the $3 \mathrm{mK}$ power reflection from the polyethylene windows (neglected after Eq. B2) gives the total estimated reflected power of $6 \pm 5 \mathrm{mK}$.

\section{B.2: Emission}

Emission properties of the reference load are summarized in Table B2. The temperatures for the glass/teflon IR-blocking windows are taken from sensors on the radiometric wall where the windows attach. The $\mathbb{R}$ heat load may heat the windows to a somewhat higher temperature.

The 78-cm diameter radiometric wall is a good approximation to free-space. We estimate emission from the false wall by convolving the far-field beam pattern with the radiometric wall, using the emissivity of a thin aluminum layer. The aluminum foil contributes $<1 \mathrm{mK}$ to $\mathrm{T}_{\mathrm{A} \text {,Load. }}$ In addition, there are two joints in the radiometric wall at the locations of the glass/teflon windows. Modelling emission from the joints as blackbody gives an upper limit to their contribution of $6 \mathrm{mK}$. We estimated the total contribution to TA,Load from emission of warm parts of the load as $13 \pm 7 \mathrm{mK}$.

The microwave absorber has an emissivity $>0.999$. Its themodynamic temperature is that of the liquid helium bath. At the ambient pressure of $481 \mathrm{~mm} \mathrm{Hg}$, helium boils at $3.771 \pm 0.002 \mathrm{~K}$. Converting to antenna temperature at $7.5 \mathrm{GHz}$ and adding the minor reflection and emission terms gives the final value for the reference temperature:

$$
\mathrm{T}_{\mathrm{A}, \text { Load }}=3.613 \pm 0.009 \mathrm{~K}
$$




\begin{tabular}{llccc}
\hline & Position (cm) & \multicolumn{1}{c}{ r } & $\Gamma$ & $\zeta(\mathrm{z})$ \\
\hline Radiometer (R) & 0.0 & 0.1 & 1.0 & --- \\
Top IR Window (G1) & 111 & $6.5 \times 10^{-3}$ & $7.9 \times 10^{-2}$ & $7 \times 10^{-3}$ \\
Bottom IR Window (G2) & 116 & $1.3 \times 10^{-2}$ & $7.9 \times 10^{-2}$ & $7 \times 10^{-3}$ \\
LHe interface (H) & 156 & $1.2 \times 10^{-2}$ & $2.1 \times 10^{-2}$ & $4 \times 10^{-3}$ \\
Absorber (A) & 162 & $3.2 \times 10^{-3}$ & $2.1 \times 10^{-2}$ & $4 \times 10^{-3}$ \\
\hline G1-G2 & --- & -- & -- & 0.9 \\
G1-H & -- & -- & -- & $5 \times 10^{-2}$ \\
G1-A & -- & -- & -- & $4 \times 10^{-2}$ \\
G2-H & -- & -- & -- & $6 \times 10^{-2}$ \\
G2-A & --- & -- & -- & $4 \times 10^{-2}$ \\
H-A & -- & -- & -- & 0.9 \\
\hline
\end{tabular}

Table B1: Properties of the reflecting surfaces within the reference load. The position is relative to the throat of the hom, taken as the reference for position and phase.

\begin{tabular}{lccc}
\hline Material & Emissivity & Temperature (K) & Emission (mK) \\
\hline Polyethylene & $2.4 \times 10-6$ & 270 & 0.7 \\
Top IR Window & $4.9 \times 10-5$ & 50 & 2.5 \\
Bottom IR Window & $9.9 \times 10-5$ & 30 & 3.0 \\
Radiometric Wall & $-10^{-4}$ & $4-270$ & $<1$ \\
Joints & 1.0 & 30,50 & 6 \\
\hline Total & & & $13 \pm 7 \mathrm{mK}$ \\
\hline
\end{tabular}

Table B2: Emission properties of the reference load. 


\section{Appendix C \\ Interferometry}

The electric field $E(r, t)$ at a point $r$ emitted from a radio source at location $R$ may be expressed in terms of its Fourier transform $E_{v}(R)$. For sources of astrophysical interest, $|\mathbf{R}|$ is large, and we may treat the emitting region as a two-dimensional distribution on the surface of a sphere, characterized by the field distribution $\varepsilon_{v}(R)$. The resultant field at the observer's location $r$ is

$$
E_{v}(\mathbf{r})=\int \varepsilon_{v}(\mathbf{R}) \frac{\exp (2 \pi \mathrm{iv}|\mathbf{R}-\mathbf{r}| / \mathbf{c})}{|\mathbf{R}-\mathbf{r}|} \mathrm{dS}
$$

where $d S$ is the element of surface area on the celestial sphere. An interferometer measures the correlation of the electric field at points $\mathbf{r}_{1}$ and $\mathbf{r}_{2}$,

$$
\begin{aligned}
V_{v}\left(\mathbf{r}_{1}, \mathbf{r}_{2}\right) & =\left\langle E_{v}(\mathbf{r}) E_{v}^{*}(\mathbf{r})>\right. \\
& =<\iint \varepsilon_{v}\left(\mathbf{R}_{1}\right) \varepsilon_{v}^{*}\left(\mathbf{R}_{2}\right) \frac{e^{2 \pi i v \mid R_{1}-\mathbf{r}_{1} / \mathrm{c}}}{\left|\mathbf{R}_{1}-\mathbf{r}_{1}\right|} \frac{e^{-2 \pi i v \mid R_{2}-r_{2} / / c}}{\left|\mathbf{R}_{2}-\mathbf{r}_{2}\right|} d S_{1} \mathrm{~d} S_{2}>
\end{aligned}
$$

where the asterisk represents complex conjugation. Radiation from most astrophysical sources is spatially incoherent,

$$
<\varepsilon_{v}\left(\mathbf{R}_{1}\right) \varepsilon_{v}^{*}\left(\mathbf{R}_{2}\right)>=0, \quad \mathbf{R}_{1} \neq \mathbf{R}_{2}
$$

and Eq. C2 simplifies to

$$
V_{v}\left(\mathbf{r}_{1}, \mathbf{r}_{2}\right)=\int<\left|\varepsilon_{v}(\mathbf{R})\right|^{2}>|\mathbf{R}|^{2} \frac{e^{2 \pi i v\left|R-r_{1}\right| / c}}{\left|R-r_{1}\right|} \frac{e^{-2 \pi i v \mid R-r_{2} / / c}}{\left|R-r_{2}\right|} d S
$$

Defining the unit vector $\mathbf{s} \equiv \mathbf{R} / \mathbf{R} \mid$ and the observed intensity $I_{V}(\mathbf{s}) \equiv|\mathbf{R}|^{2}<\left|\varepsilon_{V}(\mathbf{R})\right|^{2}>$, and neglecting terms of order $|\mathbf{r} / \mathbf{R}|$ gives the relation for the correlation

$$
V_{V}\left(r_{1}, r_{2}\right)=\int I_{V}(s) e^{-2 \pi i v s-\left(r_{1}-r_{2}\right) / c} d \Omega \text {. }
$$

For most observations, we are restricted to observing a small section of the sky (often defined by the primary beam pattern $A_{v}(s)$ of the radio telescopes used), so that we may express the unit vector $s$ in terms of the unit vector $s_{0}$ and the vector $\sigma$ perpendicular to $s_{0}$,

$$
s=s_{0}+\sigma .
$$


With the coordinate system conventionally taken such that $s_{0}=(0,0,1)$, the vector $r_{1}-r_{2}$ may be expressed in terms of unit wavelength,

$$
\mathbf{r}_{1}-\mathbf{r}_{2}=\lambda(\mathrm{u}, \mathrm{v}, \mathrm{w}) \text {. }
$$

Eq. $\mathrm{C} 4$ then becomes

$$
V_{v}^{\prime}(u, v, w)=e^{-2 \pi i w} \iint I v(l, m) e^{-2 \pi i(u l+v m)} d l d m
$$

By convention, the phase $e^{-2 \pi i w}$ is absorbed into the left side of Eq. C5 so that the correlation is independent of $w$ :

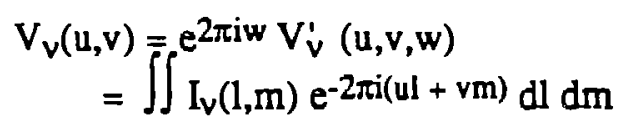

Eq. $\mathrm{C} 6$ is the coherence function relative to the phase center so. Its Fourier transform gives us the desired surface brightness $I_{v}(1, m)$ on the surface of the celestial sphere:

$$
I_{v}(l, m)=\iint V_{v}(u, v) e^{2 \pi i(u l+v m)} d u d v
$$

In practice, the surface brightness $I_{V}(s)$ is convolved with the normalized beam pattern $A_{v}(s)$ of the individual elements of the interferometer. The coherence function (Eq. C6) then becomes

$$
V_{v}(u, v)=\iint A_{v}(1, m) I v(l, m) e^{-2 \pi i(u l+v m)} d l d m
$$

and is referred to as the complex visibility. For a finite number of elements in the interferometer, the complex visibility is not known for all $(u, v)$, but is a set of discrete samples in the $u-v$ plane. Defining the sampling function $S(u, v)$ to be zero wherever no data are taken and unity otherwise, the Fourier transform of the complex visibility (Eq. C7) becomes

$$
I P(l, m)=\iint V_{v}(u, v) S(u, v) e^{2 \pi i(u l+v m)} d u d v
$$

and is referred to as the "dirty" image of the source brightness distribution. It is the true intensity convolved with the synthesized beam,

$$
B(l, m)=\iint S(u, v) e^{2 \pi i(u l+v m)} d u d v .
$$


The smallest spatial structure to which the interferometer is sensitive is given by the largest baselines in the $(u, v)$ plane, and the largest structure by the smallest baselines.

For a thorough derivation of interferometry and synthesis imaging at the VLA, the reader is referred to the NRAO Synthesis Imaging course notes (ed. Perley et al. 1985). 


\section{ACKNOWLEDGMENTS}

I have been fortunate throughout my stay at Berkeley in terms of the people with whom I have had the pleasure of working. George Smoot has been much more than an adviser, and has shaped my scientific interests and talents while teaching me to keep those projects juggled. Steve Levin and Marc Bensadoun have been ready with advice, intellectual stimulation, baseball, and the long huck. Giovanni De Amici provided a ready hand and a readier mind when the inevitable problems arose. Chris Witebsky has given invaluable aid as a sounding board for all stages of these projects.

I have enjoyed collaborating with Chuck Bennett and Sam Petuchowski at NASA's Goddard Space Flight Center. They helped introduce me to the field of radio astronomy. Sam provided the computer code for the solution of the radiative transfer problem in molecular clouds.

I am indebted to John Gibson for the superb electronic design, redesign, revised designs, and field repairs that have enabled the experiments to work at all. Hal Dougherty built most of the $10 \mathrm{GHz}$ radiometer, the $7.5 \mathrm{GHz}$ radiometer hardware was but a pale reflection of his handiwork. Jon Aymon has been a friend and co-conspirator, with a unsuspected taste for high-altitude labor (a taste shared by Luis Tenorio). Faye Mitschang has helped me through more Official Forms than I care to recall; without her I could not have successfully navigated the sea of bureaucracy into which I have been cast. You are all truly "men and women of culture and science."

One of the advantages I have enjoyed is the quality of the personnel and facilities at which the observations and analysis have taken place. The Lawrence Berkeley Laboratory is one of the nation's premier sites for scientific endeavor, I have found it an immensely stimulating workplace. The staff of the White Mountain Research Station deserve special praise for their efforts above and be $j$ ond the call of duty (and well after hours). Their resourcefulness and willingness to lend a hand enabled us to salvage more than one hypoxic snafu. The NRAO staff at both Socorro and Charlottesville provided expert assistance to a novice observer. This work has been supported by the National Science Foundation and the Deparment of Energy unde "co..zact No. DE-AC03-76SF00098.

I would like to thank my parents for their support to my academic endeavors and for instilling ir. me the ide: that knowledge is worthwhile in and of itself. Still, after 21 years, they must $b$. glad I'm finally out of school. Finally, I would like to express my heartfelt appreciatio: "n Teri, who has put up with research that has taken me to increasingly remote and desolate sites. Her patience with innumerable "half hour" delays must mean she loves me. 


\section{REFERENCES}

Adams, F.C., Freese, K., Levin, J., and McDowell, J.C. 1989, Ap. J. (submitted).

Amal, E.M., and Goss, W.M. 1985, Astron. Astroph., 145, 369.

Bartlett, J. and Silk, J. 1989, Ap. J. (submitted).

Bersanelli, M., Witebsky, C., Bensadoun, M., De Amici, G., Kogut, A., Levin, S., and Smoot, G. 1989, Ap. J., in press.

Bieging, J.H., Wilson, T.L., and Downes, D. 1982, Astron. Astroph. Suppl. Ser., 49, 607.

Cohen, R.J., Few, R.W., and Booth, R.S. 1979, Mon. Not. R. Ast. Soc., 187, 35p.

Crane et al. 1988, Proc. Third ESO/CERN Symposium, in press.

Danese, L. and Partridge, R.B. 1989, Ap. J. (in press).

-, and De Zotti, G. 1978, Astron. Astroph., 68, 157.

-, and De Zotti, G. 1980, Astron. Astroph., 84, 364.

—, and De Zotti, G. 1982, Astron. Astroph., 107, 39.

De Amici, G., Smoot., G., Friedman, S.D., and Witebsky, C. 1985, Ap. J., 298, 710.

De Amici, G., Smoot, G., Aymon, J., Bersanelli, M., Kogut, A., Levin, S., and Witebsky, C. 1988, Ap. J., 329, 556.

de Vaucoleurs, G. 1979, ApJ., 233, 433.

Donnelly, R. J. 1967, Experimental Superfluidity, University of Chicago Press, Chicago Il.

Draine, B.T., and Lee, H.M. 1984, Ap. J., 285, 89.

Evans, N.J., Zuckerman, B., Morris, G., and Sato, T. 1975, Ap. J., 196, 433.

Freese, K., Adams, F.C., Frieman, J.A., and Mottola, E. 1987, Nuc. Phys. B., 287, 797.

Friedman, S.D., Smoot, G.F., De Amici, G., and Witebsky, C. 1984, Phys. Rev. D, 29, 2677.

Friedman, S.D. 1984, Ph.D. Thesis, University of Califomia, LBL17279.

Gardner, F.F., and Whiteoak, J.B. 1984, Mon. Not. R. Ast. Soc., 210, 23. 
Garrison, B.J., Lester, W.A. Jr., Miller, W.H. and Green, S. 1975, Ap. J. Letr., 200, L175.

Genzel, R., Downes, D., Schneps, M.H., Reid, AM.J., Moran, J.M., Kogan, L.R., Kostenko, V.I., Matveyenko, L.I., and Ronnang, B. 1981, Ap. J., 247, 1039.

Goldreich, F., and Kwan, J. 1974, Ap. J., 189, 441.

Green, S., Garrison, B.J., Lester, W.A. Jr., and Miller, W.H. 1978, Ap. J. Suppl., 37,321 .

Guilbert, P.W., and Fabian, A.C. 1986, Mon. Not. R. Ast. Soc., 220, 439.

Harvey, P.M., Joy, M., Lester, D.F., and Wilking, B.A. 1986, Ap. J., 300, 737.

Haslam, C.G.T., Salter, C.J., Stoffel, H., and Wilson, W.E. 1982, Astr. Astroph. Suppl. Ser., 47, 1 .

Hayakawa, S., Matsumoto, T., Matsuo, H., Murakami, H., Sato, S., Lange, A., and Richards, P.L. 1988, Publ. Astron.Soc Japan, 39, 941.

Johnson, D. G., and Wilkinson, D. T. 1986, Ap. J. (Letters), 313, L1.

Ho, P.T.P., Genzel, R., and Das, A. 1983, Ap. J., 266, 596.

Jaffe, D.T., Becklin, E.E., and Hildebrand, R.H. 1984, Ap. J. (Letters), 279, L51.

Kogut, A., Bersanelli, M., De Amici, G., Friedman, S.D., Griffith, M., Grossan, B., Levin, S., Smoot, G.F., and Witebsky, C. 1988, Ap. J. 235, 1.

Kompaneets, A.S. 1957, Sov. Phys. JETP, 4, 730.

Kraus, J. D. 1966, Radio Astronomy, 241-244, McGraw-Hill, New York.

Levin, S., Smoot, G.F., Bensadoun, M., Bersanelli, M., De Amici, G., Kogut, A., and Witebsky, C. 1988, Ap. J. 334, 14.

Liebe, H.J. 1981, Radio Sci. 16(6), 1183.

-, 1985, Radio Sci. 20, 1069.

-, Manabe, T, and Hufford, G.A. 1988, IEEE Trans.(submitted).

Lucy, L.B. 1971, Ap. J., 163, 05.

Mandolesi, N., Caizolari, P., Cortiglioni, S., and Morigi, G. 1984, Phys. Rev. D, 29, 2680.

Mandolesi, N., Calzolari, P., Cortiglioni, S., Morigi, G. 1986, Ap. J., 310, 561.

Martin, A.H.M. 1972, Mon. Not. Roy. Ast. Soc., 194, 809.

Martin-Pintado, J., Wilson, T.L., Johnston, K.J., and Henkel, C. 1985, Ap. J., 299, 386. 
Matsumoto, T., Hayakawa, S., Matsuo, H., Murakami, H., Sato, S., Lange, A.E., and Richards, P.L. 1988, Ap. J., 329, 567.

Meyer, D.M., and Jura, M. 1985, Ap. J., 297, 119.

—, Black, J.H., Chaffee, F.H. Jr., Foltz, C.B., and York, D.G. 1986, Ap. J. (Letters), 308, L37.

-, Roth, K.C., and Hawkins, I 1989b, Ap. J. (Lett ers), (in press).

Mundy, L.S., Evans, N.J., Snell, R.L., and Goldsmith, P.F. 1987, Ap. J., 318, 392.

Ostriker, J.P., and Thompson, C. 1987, Princeton University Observatory preprint POP-232.

Partridge, R.B., Cannon, J., Foster, R., Johnson, C., Rubinstein, E., and Rudolph, A. 1984, Phys. Rev. D 29, 2683.

Perley, R.A., Schwab, F.R., and Bridle, A.H. ed. 1985, Synthesis Imaging Workshop No. 13, National Radio Astronomy Observatory, Green Bank, W.V.

Plambeck, R.L., Snell, R.L., and Loren, R.B. 1983, Ap. J., 266, 321.

Sandage, A.R., and Tammann, G. 1976, Ap. J., 210, 7.

Scott, P.F. 1978, Mon. Not. Roy. Ast. Soc., 183, 435.

Silk, J., and Stebbins, A. 1983, Ap. J. 269, 1.

Sironi, G., and Bonelli, G. 1986, Ap. J., 311, 418.

Sironi, G., P. Inzani, and Ferrari, A. 1984, Phys. Rev. D, 29, 2686.

Smoot, G., et al. 1983, Phys. Rev. Letters, 51, 1099

Smoot, G., et al. 1985, Ap. J. Lett., 291, L23.

Smoot ,G.F., Bensadoun, M., Bersanelli, M., De Amici, G., Kogut, A., Levin, S., and Witebsky, C. 1989, Ap. J. Lett., submitted.

Sunyaev, R. A., and Zel'dovich, Ya. B. 1970, Astrophys. Space Sci., 7, 20.

Sunyaev, R. A., and Zel'dovich, Ya. B. 1980, Ann. Rev. Ast. Ap., 18, 537.

Thaddeus, P. 1972, Ann. Rev. Astron. Astrophys., 10, 305.

Townes, C.H. and Cheung, A.C. 1969, Ap. J. (Letters), 157, L103.

Wadiak, E.J., Rood, R.T., and Wilson, T.L. 1988, Ap. J., 324, 931.

Webster, A. S. 1974, Mon. Not. Roy. Astr. Soc., 166, 355.

Weinberg, S. 1972, Gravitation and Cosmology (New York:Wiley) 
Weiss, R. 1980, Ann. Rev. Astron. Astrophy., 18, 489.

Wilson, W.J. Schwartz, P.R., Epstein, E.E., Johnson, W.A., Etcheverry, R.D., Morgi, T.T., Berry, G.G., and Dyson, H.B. 1974, Ap. J., 191, 357.

Witebsky, C., Smoot, G., De Amici, G., and Friedman, S.D. 1986, Ap. J., 310, 145.

Zel'dovich, Ya. B., and Sunyaev, R.A. 1969, Ap. Space Sci.,4,301. 